HOW TO USE THIS DIRECTORY

Each business is listed alphabetically with essential information about its operation, products, and services. Some businesses chose to pay for an enhanced listing that includes their name in larger print, their URL, and a more detailed description.

The location index provides an alphabetical listing of businesses by state, province, or insular area and by whether they produce plants or seeds.

Please remember that directories are out-of-date as soon as they are printed. Although precautions were taken to include only valid information, we cannot guarantee the accuracy of any given listing.

\section{THE 2010 DIRECTORY}

The basis for our directory is the database maaintained by the USDA Forest Service, Reforestation, Nurseries and Genetics Resources (RNGR) team. Beginning this year, representatives from the University of Georgia, Southern Regional Extension Forestry (SREF) will contact everyone on the list to verify information. The database is also avail- able and searchable on the RNGR website (http://www.rngr.net), which also holds a vast amount of information about growing native plants.

To report an error or omission, please contact SREF at directory@rngr.net.

In addition, each producer of native plant materials will have an opportunity to publish a free basic listing, a standard listing free to subscribers of the journal (nominal fee to nonsubscribers), or an enhanced listing for an additional fee. For more information about this service please contact Susan S Franko, Native Plant Materials Directory, PO Box 8232, Moscow ID 83843-0732; ph 208.882.2601 fax 208.882.2601.

\section{CONSIDERATIONS WHEN PURCHASING NATIVE PLANT MATERIALS}

One of the most important differences between native plant materials and ornamental nursery stock is that natives are usually outplanted in relatively harsh environments without any subsequent care. Here are a few other things to keep in mind.

\section{Source-Identified}

Native plant materials are usually source-identified, which means that the seeds or cuttings can be traced back to their collection location. Conversely, most ornamentals are introduced species that have been selected for form or color, not source of origin. The importance of proper source identification cannot be overstated-local sources are assumed to be best unless scientific data proves otherwise. It's always a good idea to ask seed and plant producers what sources they have available, rather than asking if they have a particular source in stock.

\section{Locally Adapted}

In addition to selecting the proper species and genetic source (source-identified), native plant materials must be properly acclimated or processed (hardened) so that they will survive and grow under the climatic conditions of the project site. Although the species may be "locally adapted" to grow on a particular site, if it has not been properly hardened its survival and growth will be poor. The question of whether plant materials will be hardy on the outplanting site is often overlooked. 


\section{native plant materials directory}

\section{Selecting a Nursery and (or) a Seed Producer}

Native plant customers should visit local nurseries and seed production facilities and talk to the personnel. Take a walk around the operation and look at the current crop. In nurseries, remember to check the quality of the root systems as well as the shoots. In a seed production facility, check for weeds and vitality of the plants. Ask for a customer list and search out others who have purchased seeds or other plant materials. Inquire about the reputations of your potential vendors, including the success of their materials on outplanting sites.

\section{Other Business Services}

Traditionally, nurseries and seed producers merely grew plants and seeds for sale, but the current trend is to go beyond propagation to a broader range of services. Nowadays, native plant growers offer a variety of services, depending on their facilities and expertise. An increasing number of progressive nurseries provide a full range of services, from seed collection and processing, plant propagation and storage, through outplanting and follow-up care.

\section{Communication Is Essential}

Buyers of native plant materials should consider the nursery manager or seed producer as a partner in their planting project and involve them early in the planning process.

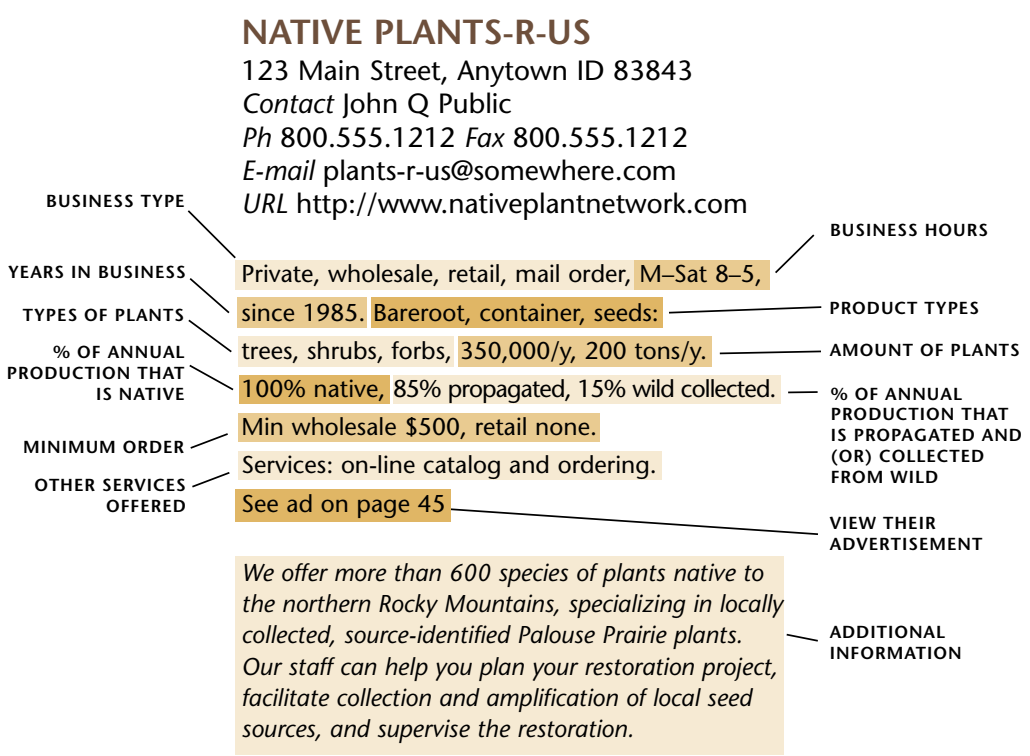




\section{E TREE FARMS AND WETLAND NURSERY INC}

PO Box 476, Loxahatchee FL 334703069

248 C Rd, Loxahatchee FL 33470

Contact Irene Goltzené

Ph 561.718.3069 Fax 888.773.4458

E-mail native3etrees@aol.com

Private, wholesale, retail, mail, Internet, contract, M-F 8-4 weekends by appointment, since 1995. Plants: $95 \%$ native, $25 \%$ propagated, $25 \%$ wild collected; container, bareroot, cuttings; tree, shrub, herbaceous, grass, wetland/aquatic.

Quality native trees, shrubs, palms, ferns, and aquatics. Let us be your one native plant stop. Delivery and contract growing are also available.

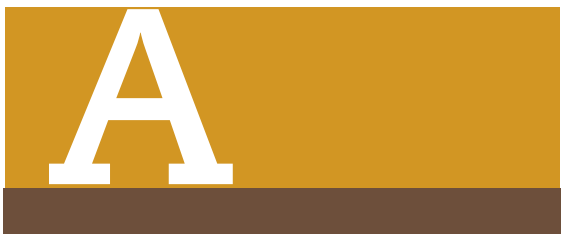

A Quality Plant 1998 19th Ave NE, Ruskin FL 33570 Contact Rob Pittman Ph 866.998.9393 Fax 813.645.8268 E-mail robp@aqualityplant.com Private, wholesale, retail, contract. Plants.

\author{
A Touch O' Green Landscape \& \\ Gardens LLC \\ 7205 Holly Fern Cove, \\ Austin TX 78750 \\ Contact Bridget \\ Ph 512.736.4859 Fax 512.349.2712 \\ E-mail toglg@sbcglobal.net \\ Private, wholesale, retail. Plants.
}

\section{A Waldbart \& Sons Nursery} 5517 N Hwy 67, Florissant MO 63034 Contact Jack Cunningham Ph 314.741.3121 Fax 314.741.3825 Private, wholesale, retail. Plants.

\section{Abbott's Nursery 2781 Scio Church Rd, Ann Arbor MI 48103 \\ Ph 734.665.8733 \\ Private, retail. Plants.}

Abraczinskas Nurseries Inc 346 Numidia Dr, Catawissa PA 17820 Contact Peter Abraczinskas Ph 570.356.2323 Fax 570.356.2366 E-mail abs@sunlink.net Private, wholesale, mail. Plants.

Abundant Life Seed PO Box 279, Cottage Grove OR 97424 Ph 541.767.9606 Fax 866.514.7333 E-mailinfo@abundantlifeseeds.com Private, retail, mail. Seeds.
The Acorn Seed 2486 Oro Quincy Hwy, Oroville CA 95966

Ph 530.534.5363

Private, retail, contract. Seeds.

\section{ACORUS RESTORATION}

\#722 6th Concession Rd, Walsingham ON NOE 1XO Contact Paul Morris Ph 519.586.2603 Fax 519.586.2447 URL http://www.ecologyart.com Private, wholesale, retail, mail, Internet, contract, W-Sun 10-5, since 1995. Plants: $300,000 / y, 100 \%$ native, $100 \%$ propagated; tree, shrub, herbaceous, grass. Seeds: 1000 $\mathrm{lb} / \mathrm{y}, 100 \%$ native, $100 \%$ propagated; tree, shrub, herbaceous, grass.

Wholesale and Retail Native Plants and Ecological Consultation. We grow high-quality wildflowers, grasses, trees, and shrubs. More than 350 species for wetland, woodland, and prairie.

We offer more than 350 species of native plants suitable for wetland, woodland, prairie, and meadow. Our trees, shrubs, and herbaceous plants are grown from seeds that we collected in an ecologically responsible manner. Come out to visit the healing, songbird, butterfly, hummingbird, and water gardens and walk our trails to see our large-scale restorations of wetlands, Carolinian Woodland and Tallgrass Prairie. We also offer many biological and ecological restoration consultation services. 


\section{alphabetical listing}

\section{ACTION THEORY LANDSCAPE AND NURSERY}

6791 SW 57 Terrace, Miami FL 33143

22401 SW 130 Ave, Goulds FL 33170

Contact Claude Roatta

Ph 305.257.2244 Fax 305.667.0266

E-mail croatta@yahoo.com

URL http://www.actiontheorynursery.com

Private, wholesale, retail, contract, $\mathrm{M}-\mathrm{F}$

7:30-5, since 1997. Plants: $25 \%$ native,

$90 \%$ propagated; container; tree, shrub,

herbaceous, grass, wetland/aquatic.

\section{Adams-Franklin Tree}

Seedlings Nursery

13512 Doerfler Rd SE,

Silverton OR 97381

910 Cascade Hwy NE, Salem OR 97301

Contact Don Doerfler

Ph 503.581.5500 Fax 503.873.2736

E-mail sales@adamsfranklinnursery.com Private, wholesale, retail. Plants, Seeds.

\section{ADKINS ARBORETUM}

12610 Eveland Rd, Ridgely MD 21660

Contact Joann Healley

Ph 410.634.2847 Fax 410.634.2878

E-mailinfo@adkinsarboretum.org

URL http://www.adkinsarboretum.org

Private, retail, M-Sun 10-4, since 1985.

Plants: $100 \%$ native, $100 \%$ propagated; container; tree, shrub, herbaceous, grass.

Adkins Arboretum is a 400-acre native garden and preserve on Maryland's Eastern Shore that promotes the conservation of native plants of the Delmarva Peninsula. The Arboretum's nursery offers more than 200 native plant species for sale on-site spring through fall.
Programs in horticulture, ecology, and arts and crafts are offered for all ages.

\section{AGRECOL CORPORATION}

10101 N Casey Rd, Evansville WI

53536

2918 Agriculture Dr, Madison WI

53718

Contact Customer Service

Ph 608.223.3571 Fax 608.223.3575

E-mail ecosolutions@agrecol.com

URL http://www.agrecol.com

Private, wholesale, retail, M-F 8-5, since 1995. Plants: $100 \%$ native, $100 \%$ propagated, $95 \%$ wild collected; container; herbaceous, grass, wetland/aquatic. Seeds: herbaceous, grass.

Largest grower of native seeds and plants in the Midwest. More than 200 species. Design and installation, maintenance service, erosion control products and services, ecological and natural areas consulting, stormwater management.

\section{See ad on page 149.}

\section{AgriCycle Farms}

PO Box 1656, Delta Junction AK 99737-1656

Ph 907.895.2076 Fax 906.895.2076

E-mailmikepurv@wildak.net Wholesale. Plants.
Agua Fria Nursery

1409 Agua Fria St, Santa Fe NM

87505-0907

Contact Bob Pennington

Ph 505.983.4831 Fax 505.983.3593

E-mail aguafrianr@aol.com

Private, retail. Plants.

'Ahakhav Tribal Preserve

26600 Mohave Rd, Parker AZ 85344

Contact Stephanie Hines

Ph 928.669.2664 Fax 928.669.8024

Tribal, wholesale. Plants.

Aikane Nursery

PO Box 459, Kapa'au HI 96755

Contact Becky Tarties

Ph 808.889.5906 Fax 808.889.0032

E-mailinfo@aikanenursery.com

Private, wholesale, retail. Plants.

Aileen's Nursery-Po'o Wai U

942 W Kawailani St, Hilo HI 96720

Contact Aileen KFW Yeh

Ph 808.936.2671 Fax 808.959.8040

E-mail ayeh@hawaii.rr.com

Private, wholesale, retail, contract. Plants.

Alabama Forestry Commission-

EA Hauss Nursery

4165 Ross Rd, Atmore AL 36502

Contact Bobby

Ph 251.368.4854 Fax 251.368.8624

E-mail haussnursery@forestry.state.

alabama.gov

State. Plants. 
Alaska Dept of Natural

Resources-Plant Materials

Center Forest Nursery

5310 Bodenburg Spur Rd,

Palmer AK 99645

Contact Stoney Wright

Ph 907.745.4469 Fax 907.746.1568

E-mail ron.cotterman@alaska.gov

State, no public sales. Plants, Seeds.

Alaska Division of Forestry

PO Box 185, 110 Mile Richardson Hwy,

Glennallen AK 99588

Contact Blair Acheson

Ph 907.822.5534 Fax 907.822.8600

E-mail blair.acheson@alaska.gov

State, no public sales. Plants.

Alaska Greenhouses Inc

1301 Muldoon Rd,

Anchorage AK 99504

Ph 907.333.6970 Fax 907.333.8330

E-mail coho@alaskalife.net

Private. Plants.

Alberta Nurseries and Seeds Ltd

Box 20, Corner of Hwy 2 and Hwy 87

secondary, Bowden AB TOM OKO

Contact Chris Bergeren

Ph 403.224.3545 Fax 403.224.2455

Private, wholesale, retail. Plants, Seeds.

ALCLA Native Plant

Restoration Inc

3208 Bearspaw Dr NW,

Calgary AB T2L 1T2

Contact Pat or Al Fedkenheuer

Ph 403.282.6516 Fax 403.282.6516

E-mail fedkenhp@telus.net or

ALCLA@telus.net

Private, retail, please call, since 1992. Plants: shrub, grass.

Container plugs are from Alberta-collected seed and are started in the greenhouse. They are available for planting throughout the season starting in May. Seed is available for some species. Experienced in the growing and planting of greenroom native plant material. Please contact us for information.

Aldrich Berry Farm \& Nursery Inc 190 Aldrich Rd, Mossyrock WA 98564

Contact Glenn Aldrich

Ph 360.983.3138

E-mail galdrich@tds.net

Private, wholesale, retail, contract, M-Sun by appt, since 1963. Plants: $25 \%$ native, $100 \%$
Aldridge Nursery Inc

PO Box 1299, Von Ormy TX 78073

10440 Jarratt Rd, Atascosa TX 78002

Contact Tom

Ph 210.622.3491 Fax 210.622.5327

E-mail aldridgenursery@mecwb.com

Private, wholesale. Plants.

Alexander Landscaping \& Plant

Farm

910 S Flamingo Rd, 830 S Flamingo Rd,

Davie FL 33325

Contact Bryan Alexander

Ph 954.472.5039 Fax 954.370.8912

Private, wholesale, retail, mail. Plants.

All Native Garden Center

\& Plant Nursery

300 Center Rd,

Fort Myers FL 33907-1513

Contact John Sibley

Ph 239.939.9663 Fax 239.936.8504

E-mail nolawn@earthlink.net

Private, wholesale, retail. Plants.

\section{All Natives}

PO Box 1045, 33601 Kieffer Rd, San

Antonio FL 33576-1045

Contact Brightman Logan

Ph 352.588.3687 Fax 352.588.4552

E-mail logan@tingley.net

Private, wholesale. Plants.

Allendan Seed

1966 175th Ln,

Winterset IA 50273-8500

Contact Dan Allen

Ph 515.462.1241 Fax 515.462.4084

E-mail allendan@allendanseed.com

Private, wholesale, M-F 8-5, since 1980.

Seeds: $100 \%$ native.

See ad on page 149.

Alpha Nurseries

3737 65th St, Holland MI 49423

Contact Jeff Busscher

Ph 269.857.7804 Fax 269.857.8162

E-mailinfo@alphanurseries.com

Private, wholesale. Plants.

Alpha Nursery Inc

5050 Hazel Green Rd NE,

Salem OR 97305

Ph 503.390.1286 Fax 503.390.2639

E-mail info@alphnursery.com

Private, wholesale. Plants.
Alpine WildSeed

1308 N Alder \#1, Ellensburg WA 98926

Contact Ken Boettger

Ph 509.933.3063

E-mailknarly@upickers.com

Private, wholesale, retail, mail, Internet, contract. Seeds.

Althouse Nursery

5410 Dick George Rd,

Cave Junction OR 97523

Contact Barbara or Graig Delbol

Ph 541.592.2395 Fax 541.592.2330

E-mail althousenursery@frontiernet.net

Private, wholesale, contract, $\mathrm{M}-\mathrm{F} 8-5$ or by

appt, since 1981. Plants: 300,000/y, 80\%

native, $100 \%$ propagated; container, cuttings.

Wholesale plug grower specializing in native trees and shrubs of the Pacific Northwest.

Contract growing available.

Amanda's Garden

8410 Harpers Ferry Rd,

Springwater NY 14560

Contact Ellen Folts

Ph 585.669.2275

E-mail amandasgarden@frontiernet.net

Private, wholesale, retail, mail. Plants.

American Grass Seed Producers

Tangent OR 97389

Contact Randy Waldie

Ph 541.926.4611 Fax 541.926.5038

E-mail prairie@dswebnet.com

Private. Seeds.

AMERICAN NATIVE PLANTS

PO Box 158, 4812 E Joppa Rd,

Perry Hall MD 21128

Contact Roy List or Michael McConnell

Ph 410.529.0552 Fax 410.529.3883

E-mailmdn@qis.net

URL http://www.americannativeplants.net Private, wholesale, retail, Internet, contract, M-F 8-5 or by appt, since 1998 . Plants: $500,000 / y, 100 \%$ native, $100 \%$ propagated; container, $B \& B$, bioengineering; tree, shrub, herbaceous, grass, wetland/aquatic.

We offer a wide variety of pioneer and early successional woody and herbaceous plants to ensure maximum viability in environmental projects. Our plants are container grown in sizes from plugs through 5-gallon containers with some balled and burlapped for larger trees. We have contract grown for such projects as the Native American building at the Smithsonian Institute and our plant inventory includes materials for restoration and landscape projects from New England to Florida and from the Atlantic seaboard to the 

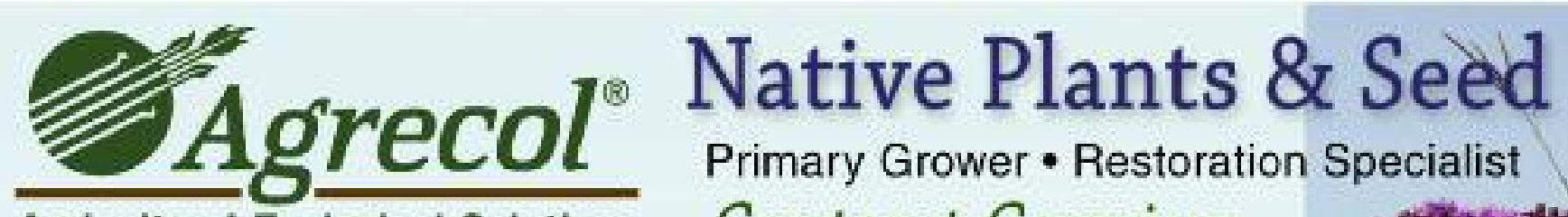

\section{Primary Grower $\bullet$ Restoration Specialist}

Agricultural Ecological Solutions Contract Growing
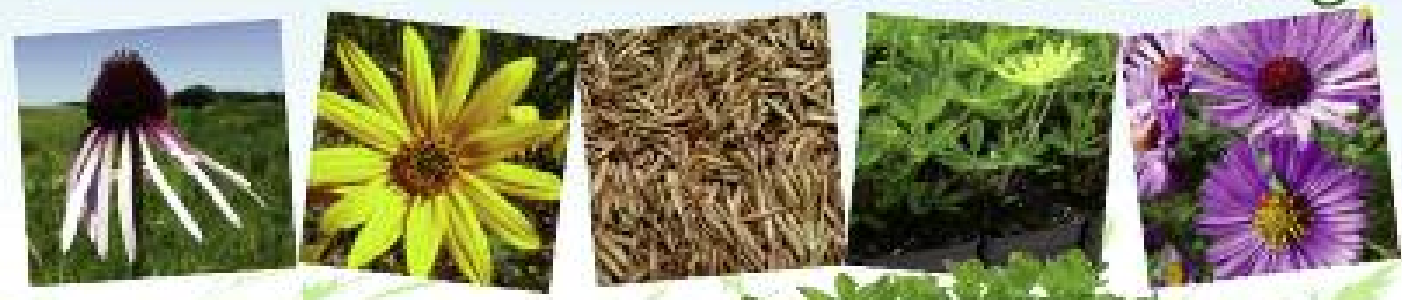

- Live Native Plants from the Agrecol Greenhouse

- Source-Certified Native Seed, sold PLS

\section{Custom Seed Mixes}

Call 608.223.3571 for quotes and availability www.agrecol.com
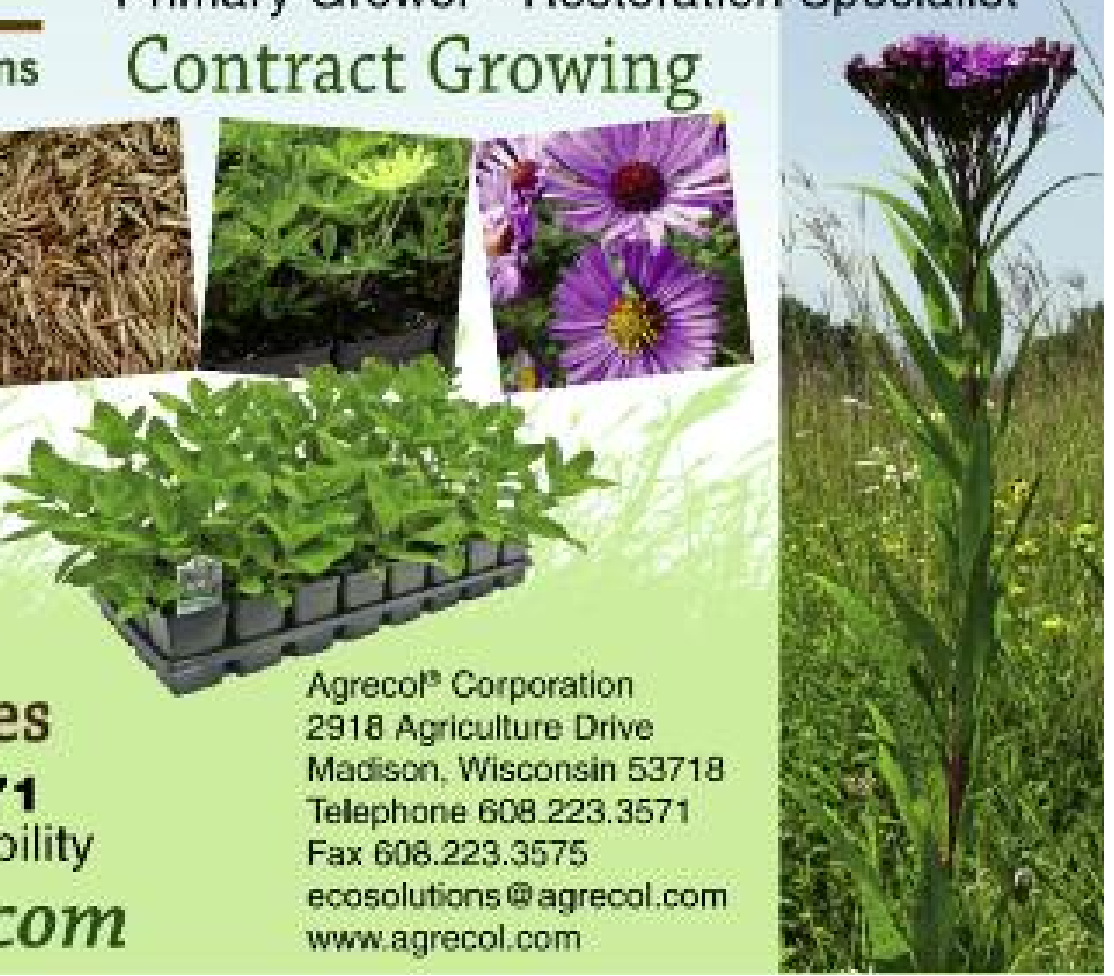

Midwest. Fax us for a quote. Catalog available by mail and online. We can ship or deliver anywhere.

American Native Products

PO Box 549, Scottsmoor FL 32775

Ph 321.383.1967 Fax 321.383.4150

E-mail buzbeefalo@yahoo.com

Private, wholesale. Plants.

American Samoa Community College-

Land Grant Forestry Extension

PO Box 5319, Pago Pago AS 96799

Contact Mike Misa

Ph 684.699.1394 Fax 684.699.5011

E-mail ssuemann@yahoo.com

Private. Plants.

AmericanMeadows.com

223 Ave D, Ste 30, Williston VT 05495

Contact Mike Lizotte

Ph 802.951.5812 x19 Fax 802.951.9089

E-mailmike@americanmeadows.com

Private. Seeds.
Agrecol ${ }^{3}$ Corporation Madison. Wisconsin 53718

Telephone 608.223.3571

Fax 608.223 .3575

ecosolutions 1 agrecol.com www.agrecol.com 
Amy Greenwell Ethnobotanical

Gardens Nursery

PO Box 1053, 826188 Mamalahoa

Hwy, Captain Cook HI 96704

Contact Brian Kiyabu

Ph 808.323.3318 Fax 808.323.2394

E-mail brian.kiyabu@bishopmuseum.org

Private, retail. Plants.

Andrews Nursery Florida

Division of Forestry

PO Drawer 849, Chiefland FL 32644

9850 NW 42nd Ct, Chiefland FL 32626

Contact Steven Gilly

Ph 352.493.6096 Fax 352.493.6084

E-mail gillys@doacs.state.flu.us

State, wholesale. Plants.

Angelica Nurseries Inc

11129 Locust Grove Rd,

Kennedyville MD 21645

Contact Kim Usilton

Ph 410.928.3111 Fax 410.928.3044

E-mail kusilton@angelicanurseries.com

Private, wholesale. Plants.

Anna Nursery

PO Box 1, Cobden IL 62920

Ph 618.893.2124 Fax 618.893.2125

Private, wholesale. Plants.

Antelope Valley Resource

Conservation District Tree

Nursery \& Arboretum

10148 W Ave I, Lancaster CA 93536

Contact Thomas Florence

Ph 661.942.7306 Fax 661.942.3055

E-mail treesbyme@excite.com

Federal, wholesale, retail. Plants.

Apalachee Native Nursery

12 Hayfield Spur, Monticello FL 32344

Contact William Dickerson

Ph 850.997.8976

E-mail atalnative@aol.com

Private, wholesale, retail, M-F 8-5, since

1981. Plants: $80 \%$ native, $100 \%$ propagated.

Appalachian Native Plants Inc

676 Waddell Rd,

Mountain City TN 37683

Ph 423.727.4264

E-mail appnativeplants@gmail.com

Private, wholesale, retail, contract. Plants.
Appalachian Nurseries Inc

1724 Clay Hill Rd,

Chambersburg PA 17202

Contact Tom McCloud

Ph 717.597.0066; 877.743.4733

Fax 717.597.9073

E-mail liners@appnursery.com

Private, wholesale. Plants.

Applewood Seeds

5380 Vivian St, Arvada CO 80002

Contact Norm Poppe

Ph 303.431.7333 Fax 303.467.7886

E-mail sales@applewoodseed.com

Private, wholesale. Plants, Seeds.

\section{APPLIED ECOLOGICAL SERVICES} TAYLOR CREEK RESTORATION

NURSERY

PO Box 256, 17921 Smith Rd, Brodhead WI 53520-0256

Contact Customer Service

Ph 608.897.8641 x33

Fax 608.897.2044

E-mail tcrn@appliedeco.com

URL http://www.appliedeco.com

Private, wholesale, retail, since 1985. Plants: $700,000 / y, 100 \%$ native, $100 \%$ propagated; container; herbaceous, grass, wetland/aquatic. Seeds: $20,000 \mathrm{lb} / \mathrm{y}, 100 \%$ native, $80 \%$ propagated, $20 \%$ wild collected; herbaceous, grass.

Nationwide native nursery, ecological contracting, and ecological consulting services.

With every order you have access to the knowledge of experienced scientists and technicians who can help you avoid costly mistakes and remedial work. Because of our passion for restoration, we want your project to succeed.

See ad on page 151.

A'Qam Native Plant Nursery c/o Ktunaxa Kinbasket Treaty Council, 7468 Mission Rd,

Cranbrook BC V1C 7E5

Contact Garry Graham

E-mail aqam@rockies.net

Tribal. Plants.

AQUASCAPES UNLIMITED INC

PO Box 364, Pipersville PA 18947

Contact Randy Heffner

Ph 215.766.8151 Fax 215.766.8986

E-mail wetland@comcat.com

URL http://www.aquascapesunlimited.com

Private, wholesale, contract, since 1985.

Plants: $60 \%$ native, $100 \%$ propagated; container, bareroot.
Container, bareroot, plugs, liners; herbaceous aquatic and wetland species, some woody. Services: contract growing, seed collection, aquatic and wetland consultation. Aquascapes Unlimited Inc is the most complete wholesale source of herbaceous wetland and ornamental aquatic perennials in the northeastern US. Aquascapes provides seed, plug, quart, and gallon materials to landscape contractors, mitigators, restorationists, and garden centers. Seed grown, unusual, and hard-to-find items including native and hybrid Sarracenias are available.

Aquatic and Wetland Company 9999 Weld Co Rd 25, Fort Lupton CO 80621

Contact Jim Meining

Ph $303.442 .4766 \times 120$

Fax 303.857.2455

E-mail jimm@aquaticandwetland.com Private, wholesale, M-F 8-5, since 1994. Plants: $90 \%$ native, $90 \%$ propagated, $15 \%$ wild collected.

Aquatic Nursery

38 W 135 McDonald Rd,

Elgin IL 60124

Contact Robert D Steinbach

Ph 847.741.7678

E-mail rdsteinbach@hotmail.com

Private, wholesale, retail. Plants.

\section{AQUATIC PLANTS OF}

FLORIDA INC

8120 Blaikie Ct, Sarasota FL 34240

Contact Gilbert J Sharell or Jessica Friday

Ph 941.378.2700 Fax 941.378.0020

E-mail info@aquaticplantsofflorida.com

URL http://www.aquaticplantsofflorida.com Private, wholesale, mail, contract, 8-5:30, since 1994. Plants: container, bareroot, B\&B, cuttings, bioengineering; tree, shrub, herbaceous, grass.

Wholesale grower and installer of native plants, trees, and grasses. Offers sea oats, dune vegetation, and aquatic plants. Aquatic Plants of Florida is a certified wetland farm where it grows more than 135 species of native plants. The company specializes in providing various freshwater wetland and salt-tolerant species for restoration projects throughout the southeastern US and Caribbean. The company has secured through contracts and permits thousands of acres of wetlands for plant and seed harvesting. Inventory in its ponds exceeds 5 million plants. In addition, the company has an inventory of 100,000 wetland and upland trees and more than one million potted wetland grasses. These plants are propagated from seed, cuttings, and division. For more information, please contact us at 800.266.1272. Certified Wetland Farm.

See ad on page 151 . 
Aquatic Resources \& Glacial Pond Farms

Plainview Ridge, Boscobel WI 53805

Contact Ran Atkinson

Ph 608.778.1131

E-mail aqresglclpndfrms@aol.com

Private, wholesale, retail, mail. Plants.

Aquatic Systems \& Resources

PO Box 1462, Palm City FL 34991

5453 SW Quail Hollow St, Palm City FL 34990

Contact Pat Faehnle

Ph 772.286.9376

E-mail asrinc1@bellsouth.net

Private, wholesale, M-F 9-4, by appt, since 1987. Plants: wetland.

ArborGen-Alabama SuperTree Nursery

264 Co Rd 888, Selma AL 36703-8576

Contact Larry Foster

Ph 334.872.5452 Fax 334.872.2358

E-mail Ihfoste@arborgen.com

Private, wholesale, retail. Plants.

ArborGen-Bellville SuperTree Nursery

PO Box 56, Bellville GA 30414

6482 Hwy 169, Claxton GA 30417

Contact George Lowerts

Ph 912.739.4721 Fax 912.739.9409

E-mail george.lowerts@arborgen.com

Private, wholesale, retail. Plants.

ArborGen-Forest Resources Hermon Nursery

867 Blackstream Rd, Hermon ME 04401

Ph 207.848.3347 Fax 207.848.0825

Private, wholesale, retail. Plants.

ArborGen-Fred C Gragg SuperTree Nursery

186 Nevada 420, Bluff City AR 71722

Contact Bill Abernathy

Ph 800.222.1270 x21 Fax 870.685.2825

E-mail bill.abernathy@arborgen.com

Private, wholesale, retail, contract. Plants.

ArborGen-Georgia SuperTree Nursery

780 Super Tree Ln, Shellman GA 39886-9757

Contact Robert Cross

Ph 800.554.6550 Fax 229.679.5628

E-mail robert.cross@arborgen.com

Private, wholesale, retail. Plants.

ArborGen-

Livingston SuperTree Nursery

3535 Nursery Rd, Livingston TX 77351

Contact Lux Davis or Shannon Stewart

Ph 877.600.8015 Fax 936.563.2027

E-mail Isdavis@arborgen.com or

shannon.stewart@ipaper.com

Private, wholesale, retail, contract. Plants.
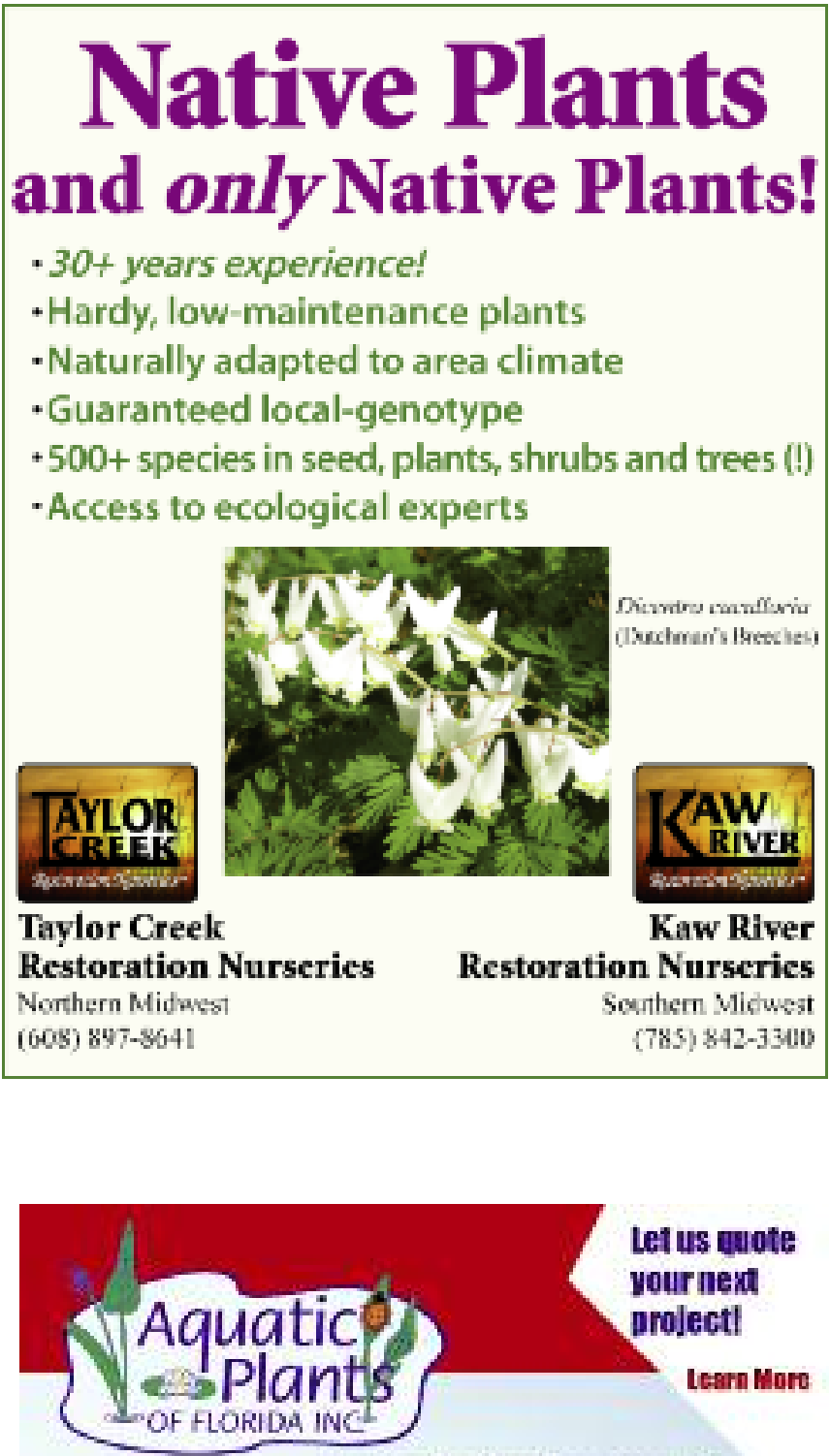

Serviag the Southeastern us

Field Grown Herbaceous Plants

liners \& Containers

Habitat Restoration Services

Pond a Mitigation Installations

Contract Growers

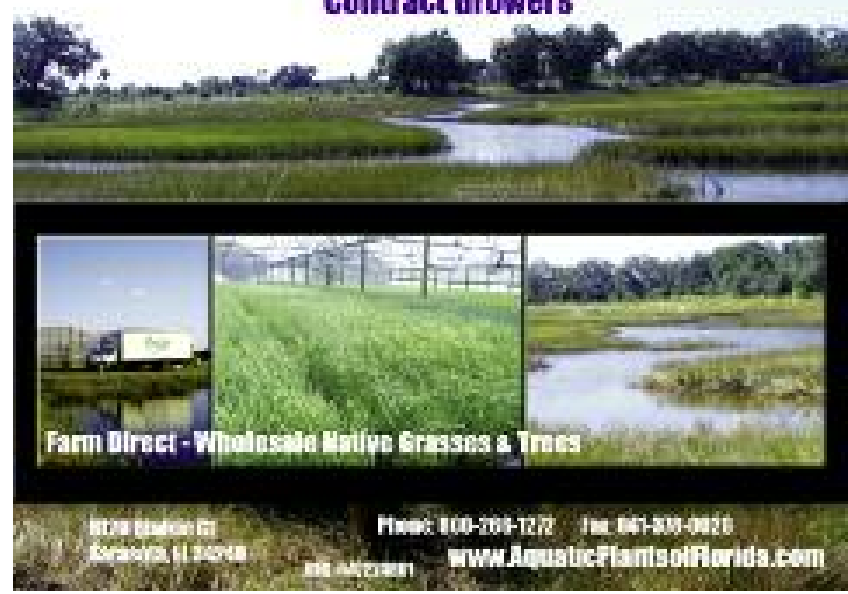


ArborGen-Richard O Barhan

SuperTree Nursery

PO Box 947, 1235 SN 3198,

Bullard TX 75757

Contact Beverly Peoples

Ph 800.642.2264 Fax 903.825.2876

E-mail beverly.peoples@arborgen.com Private, wholesale, retail, mail, contract. Plants.

ArborGen-South Carolina

SuperTree Nursery

5594 Hwy 38 S, Blenheim SC 29516

Contact Gary Nelson

Ph 800.222.1290 Fax 843.528.3943

E-mail gary.nelson@arborgen.com

Private, wholesale, retail. Plants.

ArborGen-SuperTree Orchards

4189 Bellamy Bridge Rd,

Marianna FL 32446

Contact Timothy Slichter

Ph 850.594.6001 Fax 850.594.3512

E-mail Timothy.Slichter@arborgen.com

Private, wholesale, retail. Plants.

Arid Solution LLC

PO Box 167, 3815 E Southern Ave, Phoenix AZ 85040

Contact Richard or Bob Underwood Ph 602.437.5194 Fax 602.437.4719

E-mail general@aridsolutions.com

Private, wholesale. Plants.

Arid Zone Trees

PO Box 167, Queen Creek AZ 85142

9750 E Germann Rd, Mesa AZ 85212

Contact Todd Power

Ph 480.987.9094 Fax 480.987.9092

E-mail todd@aridzonetrees.com

Private, wholesale. Plants.

Arizona Botanical

PO Drawer 160, 1601 Hwy 89-A,

Clarkdale AZ 86324

Contact Jason Lavelle

Ph 928.634.2166

Private, retail. Plants.

Arizona Native Plant Specialists I

A Great Southwest Cactus LLC

4150 E Lonemountain Rd,

Phoenix AZ 85003

Contact Bart Chrader

Ph 480.483.8355 Fax 480.948.0312

E-mail gswcactus@cox.net

152
Arizona's Best Native Plant

Care Inc

13901 N 73rd St, Ste 214,

Scottsdale AZ 85260

Contact Don Cannon or Matt Peak

Ph 480.948.7501 Fax 480.948.7401

E-mail azbest@azbest.phxcoxmail.com

Private, contract. Plants.

Arkansas Forestry Commission-

Baucum Nursery

1402 Hwy 391 N,

North Little Rock AR 72117

Contact Alan Murray

Ph 501.907.2485; 888.457.4221

Fax 501.907.2487

E-mail baucumnursery@arkansas.gov

State, wholesale. Plants.

Arkansas Valley Seed Solutions

4333 Hwy 66, Longmont CO 80504

Contact Paul Hahn

Ph 877.907.3337; 877.957.3337

Fax 303.320.7516

E-mail pdhahn@seedsolutions.com

Private, wholesale, retail, since 1945 . Seeds.

Arneson's Nursery

N11164 Hwy 45, Clintonville WI 54929

Contact Bill or Alice

Ph 715.823.6784 Fax 715.823.7124

E-mail arnesontrees@frontiernet.net

Private, wholesale, M-Sat 7-5, since 1977.

Plants: $198,000 / y, 11 \%$ native, $100 \%$ propagated.

Aroostook Band of Mic Macs

8 Northern Rd, Presque Isle ME 04769

Contact David Macek

Ph 207.764.7219 Fax 207.764.7768

E-maildmacek@micmac.nsn.gov

Tribal, wholesale. Plants.

Arrow Seed Company Inc

PO Box 722, 126 North 10th Ave, Broken Bow NE 68822

Contact Jim Girardin

Ph 800.622.4727 Fax 308.872.6945

Private, wholesale, retail. Seeds.

Arrowhead Alpines

PO Box 857, 1310 N Gregory Rd,

Fowlerville MI 48836

Contact Bob or Brigitta Stewart

Ph 517.223.3581 Fax 517.223.8750

E-mail danielapresley@hotmail.com

Private, wholesale, retail. Plants.
Arrowwood Native Plant

Nursery LLC

961 Clark Ave, Franklinville NJ 08322

Contact Cathy Arsenal

Ph 856.697.6045 Fax 856.697.6050

E-mailnjplants@aol.com

Private, wholesale. Plants, Seeds.

Arvida Nurseries

PO Box 1508, 14450 SW 312th St, Homestead FL 33090-1508

Contact Bob Plyler

Ph 800.884.9573; 305.245.9573

Fax 305.247.9549

E-mail arvida@bellsouth.net

Private, wholesale. Plants.

AT James Tree Company

3562 W Wapato Rd, Wapato WA 98951

Contact Cindy James

Ph 509.728.8535 Fax 509.877.7132

Private, wholesale, retail. Plants.

ATS PARTNERS LLC

PO Box 1306, 2514 Vada Rd,

Bainbridge GA 39817

Contact Chuck Whittaker

Ph 229.246.2662 Fax 229.248.1108

E-mail cwhittakerj@@yahoo.com

URL Call for website

Private, wholesale, 7-3:30, since 1997.

Plants: $100 \%$ native, $95 \%$ propagated, $50 \%$ wild collected. Seeds. Min: $\$ 250$.

See ad on page 153.

Aurora Nursery Inc

22821 Boones Ferry Rd NE,

Aurora OR 97070

Contact Tim Vande Kamp

Ph 503.678.7903 Fax 503.678.7901

E-mail sales@auroranursery.com

Private, wholesale, contract. Plants.

AV Seeds

4333 Hwy 66, Longmont CO 80504

Ph 877.907.3337 Fax 970.535.4522

Private. Seeds. 


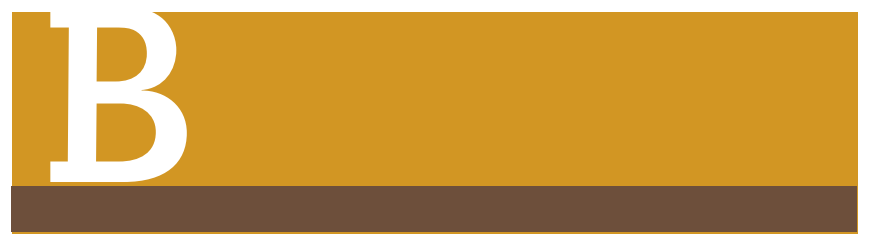

Bach's Cactus Nursery Inc

8602 N Thornydale Rd, Tucson AZ 85742

Contact Dan or Diane Bach

Ph 520.744.3333 Fax 520.742.1254

Private, wholesale, retail. Plants.

Bailey Seed Company Inc

PO Box 12788, Salem OR 97309

Contact Troy

Ph 800.407.7713; 503.362.9700 Fax 503.362.1705

E-mail troy@baileyseed.com

Private, wholesale. Seeds.

Baker's Acres

674 W Selina Ln, Wasilla AK 99654

Contact Ed Baker

Ph 907.357.4175

Private, wholesale, retail. Plants.

\section{BALANCE RESTORATION NURSERY LLC}

27995 Chambers Mill Rd, Lorane OR 97451-9707

Contact Jeff Levy

Ph 541.942.5530 Fax 541.942.7265

E-mail balancenursery@yahoo.com

Private, wholesale, contract, M-F 8-5, since 1990. Plants: $100 \%$

native, $100 \%$ propagated.

Bareroot mail order native wetland restoration nursery carries both herbs and woody plants for streamside and some upland vegetation. Sedges, rushes, bulrush, waterlily. No store, phone/fax only.

See ad on page 153.

Bamert Seed Company

1897 Co Rd 1018, Muleshoe TX 79347

Contact Nick Bamert

Ph 806.272.5506 Fax 806.272.3114

E-mail nbamert@bamertseed.com

Private, wholesale, retail, mail, Internet, contract, M-F 8-5 Sat 8-12, since 1952 . Seeds: $90 \%$ native, $100 \%$ propagated. Min: $10 \mathrm{lb}$.

Custom blends, we carry more than 40 native species.

BankSavers Nursery

PO Box 277, Arlington WA 98223-0277

Contact Dave Steiner

Ph 360.629.4960 Fax 360.629.4235

E-mail dsteiner@stillaguamish.com

Private, wholesale. Plants.
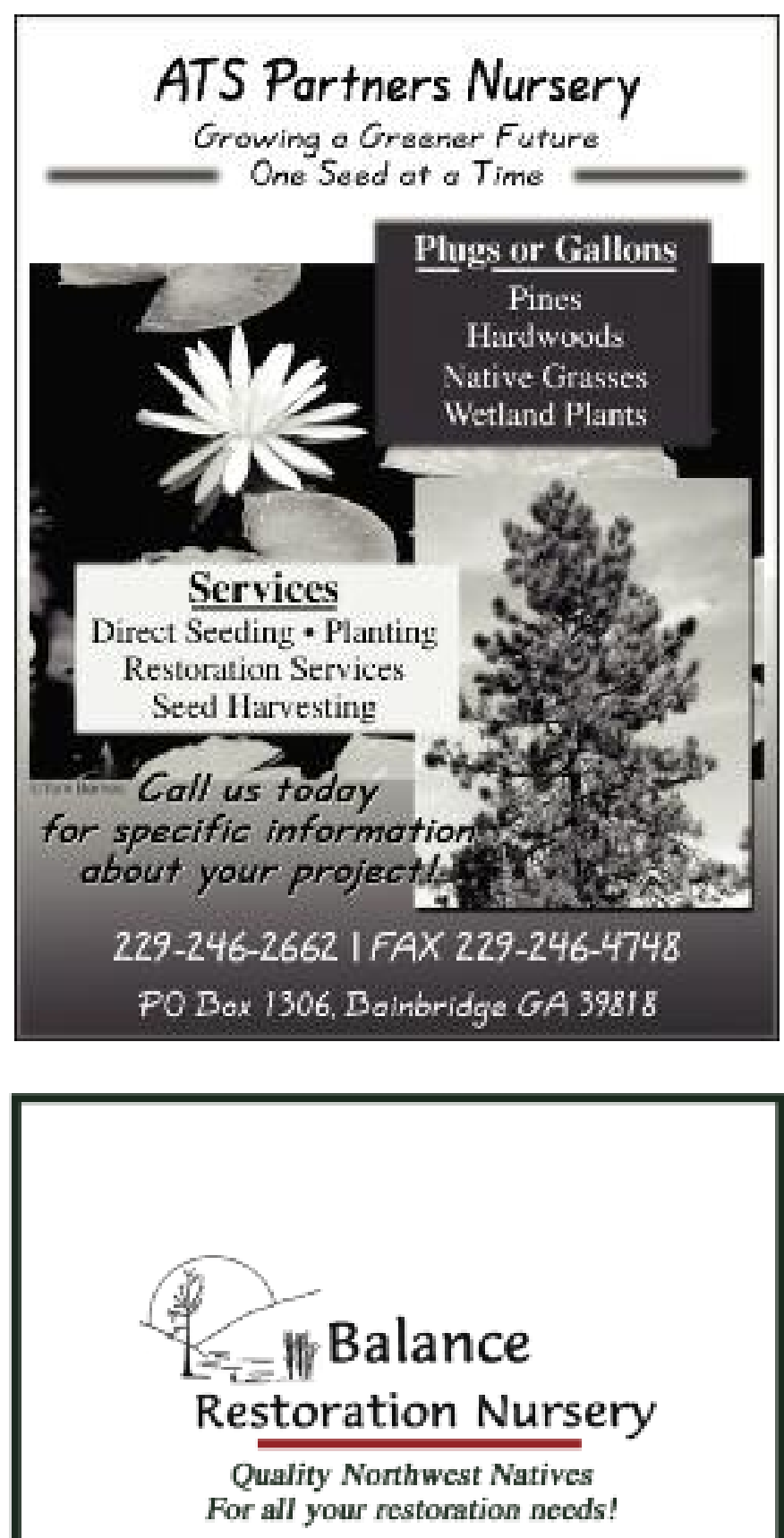

\section{Look for us on FaceBook}

Call us today

541.942 .5530 / fax 541.942 .7265

27995 Chambers Mill Road Lorane OR 97451 
Barth Nursery

8456 May Rd NE, Mt Angel OR 97362

Contact Wade Barth

Ph 503.845.6955 Fax 503.845.6955

E-mail butsch@mountangel.net

Private, wholesale. Plants.

Barton Springs Nursery

3601 Bee Cave Rd, Austin TX 78746

Contact Bernadine

Ph 512.328.6655 Fax 512.328.6517

Private, retail. Plants.

Bartow Ornamental Nursery

3890 Hwy 60 E, 200 Andy Cemetery

Rd, Bartow FL 33830

Contact Ken Ford

Ph 888.534.1350; 863.534.1350 Fax

863.534.1356

E-mail ken@southeasttrees.com

Private, wholesale. Plants.

Bate's Plants

5001 W Angle Rd, Fort Pierce FL 34945

Contact Chad Bates

Ph 561.358.7480 Fax 772.468.2525

E-mail cbatesplants@aol.com

Private, wholesale. Plants.

Bay Natives

375 Alabama St, Ste 440, San Francisco

CA 94110

Contact Paul Furman

Ph 415.287.6755 Fax 415.285.2240

E-mail info@baynatives.com

Private, wholesale, retail. Plants.

BC Nursery

4183 State Rt 276, Batavia OH 45103

Contact Chris Daeger

Ph 513.724.9032

E-mail bc4trees@fuse.net

Private, wholesale, retail, mail. Plants.

BC's Wild Heritage Plants

47330 Extrom Rd,

Chilliwack BC V2R 4V1

Contact Lee Larkin

Ph 604.858.5141 Fax 604.858.5141

E-mail bcwild@uniserve.com

Private, wholesale, retail. Plants.
Beauty Beyond Belief

3307 S College Ave, Ste 104,

Fort Collins CO 80525

Contact Don Eversole

Ph 970.204.0596 Fax 970.204.0821

Private, wholesale. Seeds.

BeaverLake Nursery

21200 S Ferguson Rd,

Beavercreek OR 97004

Contact Troy or Kelly Martin

Ph 503.632.4787 Fax 503.632.5412

E-mailinfo@beaverlakenursery.com

Private, wholesale, mail, contract. Plants.

Bechedor Inc

1508 25th Ave,

Saint-Prosper QC GOM 1YO

Contact Nicole Doyon

Ph 418.594.8580 Fax 418.594.6171

E-mail bechedor@globetrotter.net

Private, wholesale. Plants.

Beeman's Nursery Inc

3637 SR 44,

New Smyrna Beach FL 32168

Contact Steve Beeman

Ph 386.428.8889; 386.767.6232

Fax 386.428.8879

E-mail beemansnursery@att.net

Private, wholesale. Plants.

Behmerwald Nursery

4904 Garges Rd, Schwenksville PA 19473

Ph 610.287.0480 Fax 610.287.4326

E-mail behmerwald@aol.com

Private, wholesale, retail. Plants.

The Behnke Nurseries Company

11300 Baltimore Ave,

Beltsville MD 20705

Contact Alfred Millard

Ph 301.937.1100 Fax 301.937.8034

Private, retail. Plants.

Beineke's Nursery

513 Sharon Rd,

West Lafayette IN 47906

Contact Walter Beineke

Ph 765.463.2994

E-mail wbein2@verizon.net

Private, retail. Plants.
Bell Brothers Inc

PO Box 128, Bellville GA 30414

5819 Hwy 169 S, Claxton GA 30417

Contact Danny or Gary Bell

Ph 912.739.2273 Fax 912.739.2205

E-mail bellbrothers@bellsouth.net

Private, wholesale. Plants.

Bella Flora

111 Heights Blvd, Houston TX 77007

Contact Karen

Ph 713.426.5556 Fax 713.426.5558

E-mail karend7266@aol.com

Private, retail. Plants, Seeds.

Bernado Beach Native Plant Farm

1 Sanchez Rd, Veguita NM 87067

Contact Roland Phillips

Ph 505.345.6248 Fax 505.345.6248

E-mail judphil@nmia.com

Private, wholesale, retail. Plants.

Bert Driver Nursery

PO Box 351, 3400 Nashville Hwy,

Smithville TN 37166

Contact Betsy

Ph 615.597.9560 Fax 615.597.9861

E-mail bert@bertdrivernursery.com

Private, wholesale, retail. Plants.

Better Forest Tree Seeds

13872 Alan Seeger Rd,

Petersburg PA 16669

Contact Chris Kiratzis

Ph 814.667.3666 Fax 814.667.3134

E-mail tuckaway1@juno.com

Private, wholesale. Seeds.

BFI NATIVE SEEDS

$1145 \mathrm{~S}$ Jefferson Ave,

Moses Lake WA 98837

Contact Jerry Benson

Ph 509.765.6348 Fax 509.764.9978

E-mail jbenson@bfinativeseeds.com

URL http://www.bfinativeseeds.com

Private, wholesale, retail, contract, 7-5, since 1996. Seeds: $100 \%$ native, $95 \%$ propagated, $5 \%$ wild collected; herbaceous, grass.

Professional and individualized production of all native grasses and forbs for restoration projects of the Northwest. Our motto "Quality seed through genetic preservation."

See ad on page 155 . 
Biddles Nursery

PO Box 259, 1259 Hwy 89A, Sedona AZ 86336

Contact Barry Maketansky

Ph 928.282.5078 Fax 928.282.5098

Private, retail. Plants.

Big Sioux Nursery Inc

16613 Sioux Conifer Rd, Watertown SD 57201

Contact Blaine Martian

Ph 605.886.6806 Fax 605.886.7951

Private, wholesale. Plants.

\section{Big Trees Today}

PO Box 1402, 4820 SW Hillsboro Hwy, Hillsboro OR 97123

Contact Terry

Ph 503.640.3011 Fax 503.640.2877

E-mail sales@bigtreestoday.com

Private, wholesale, retail. Plants.

\section{Billings Nursery \& Landscaping}

7900 S Frontage Rd, Billings MT 59101

Contact Richard Marble

Ph 406.656.2410 Fax 406.652.6968

E-mail rmrbl@aol.com

Private, retail. Plants.

Biosphere Consulting Inc

14908 Tilden Rd,

Winter Garden FL 34787

Contact Jim Thomas

Ph 407.656.8277 Fax 407.656.2889

E-mail biosphere@biospherenursery.com

Private, wholesale, retail, M-Sa 9-5, since 1991. Plants: bareroot;

wetland. Seeds: wetland. Min: $\$ 2.00 / p k$.

\section{Birch Creek Nursery}

14060 Big Fir Dr, Prince George BC V2N 5B5

Contact Barbara Rayment

Ph 250.964.6684

E-mail birchcreek@telus.net

Private, retail, contract. Plants: $15 \%$ native, $50 \%$ propagated, $50 \%$ wild collected. Seeds.

\section{Black Creek Nursery}

1524 Mercado Ave,

Coral Gables FL 33146

13801 SW 232 St, Goulds FL 33170

Contact Pieter

Ph 305.258.2618 Fax 303.258.2633

E-mail blackcreeknsy@aol.com

Private, wholesale. Plants.

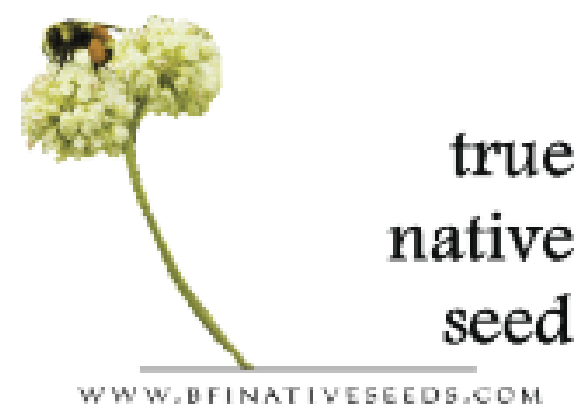

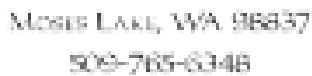

\section{directory key}

NATIVE PLANTS-R-US

123 Main Street, Anytown ID 83843

Contact John Q Public

Ph 800.555.1212 Fax 800.555.1212

BUSINESS E-mail plants-r-us@somewhere.com

TYPE URL http://www.nativeplantnetwork.com BUSINESS

BUSINESS

TYPES of Private, wholesale, retail, mail order, M-Sat 8-5

PLANTS Since 1985. Bareroot, container, seeds: — PRODUCt TYPES

$\%$ of trees, shrubs, forbs, 350,000/y, 200 tons/y. __ AMOUNT

ANNUAL
PRO-
UCTION

DUCTION

THAT

IS NATIVE

$/$

Services: on-line catalog and ordering. \% OF ANNUAL

See ad on page 45 PRODUCTION THAT IS PROPAGATED AND (OR)

MINIMUM

ORDER

OTHER

We offer more than 600 species of plants native to

SERVICES

the northern Rocky Mountains, specializing in locally collected, source-identified Palouse Prairie plants. Our staff can help you plan your restoration project, facilitate collection and amplification of local seed sources, and supervise the restoration.

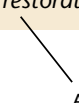
ADDITIONAL
INFORMATION 
Blackfeet Community

College Greenhouse

PO Box 819, Browning MT 59417

Contact Wilbert Fish

Ph $406.338 .5441 \times 768$

Fax 406.338.3272

E-mail Wilbert_fish@bfcc.org

Private, wholesale, retail. Plants.

\section{BLACKFOOT NATIVE}

PLANTS NURSERY

PO Box 761, Potomac MT 59823

Contact Kathy Settevendemie

Ph 406.244.5800 Fax 406.244.5800

E-mail kathy@blackfootnativeplants.com URL http://www.blackfootnativeplants.com

Blanchette Gardens

267 Rutland St, Carlisle MA 01741

Contact Pamela Blanchette

Ph 978.369.2962

Private, retail. Plants.

Blank's Nursery, Garden

\& Pet Center

448 E 500 S, La Porte IN 46350

Ph 219.393.5414

Private, wholesale, retail, mail. Plants.

Blazing Star Associates

2107 Edgewood Dr,

Woodstock IL 60098

Contact Caron Wenzel

Ph 815.338.4716 Fax 815.338.4716

E-mail cwenzel@blazing-star.com

Private, wholesale, retail. Plants, Seeds.

Blight Native Seeds Ltd

Box 244, SE 31-11-4W,

Oakville MB ROH OYO

Contact Jim Blight

Ph 204.267.2686 Fax 204.267.2699

E-mail jim@bnsltd.ca

Private, wholesale, retail, mail, Internet.

Seeds.

Bloom River Gardens

39744 Deerhorn Rd,

Springfield OR 97478

Ph 541.726.8997 Fax 541.726.4052

E-mail plants@bloomriver.com

Private, wholesale, contract. Plants.
Bloomers Flowering

Plant Nursery

5480 96th Ave, Pinellas Park FL 33782

Contact Mary Coleman

Ph 727.536.0545; 727.575.9952

E-mail bloomers7880@aol.com

Private, retail. Plants.

Bluebird Nursery

519 Bryan St, Clarkson NE 68629

Contact Wanda Wulf

Ph 800.356.9164 Fax 402.892.3738

E-mail sales@bluebirdnursery.com

Private, wholesale. Plants.

Bluestem Farm

S 5920 Lehman Rd, Baraboo WI 53913

Ph 608.356.0179

E-mail bluestem_farm@juno.com

Private, wholesale, retail, contract. Plants.

Bluestem Nursery

4001 Curry Rd, Arlington TX 76017

Contact John Snowden

Ph 817.466.2271 Fax 817.466.2293

E-mail1 1grassman@bluestemnurserycom

Private, wholesale. Plants.

\section{BLUESTEM NURSERY}

PO Box 239, Laurier WA 99146

1946 Fife Rd,

Christina Lake BC VOH 1E3

Contact Jim

Ph 250.447.6363 Fax 250.447.6363

E-mailinfo@bluestem.ca

URL http://www.bluestem.ca

Private, wholesale, retail, mail, M-F 9-5, since 1993. Plants: 100,000/y, $10 \%$ native, $100 \%$ propagated; container, bareroot, $B \& B$, cuttings; tree, shrub, herbaceous, grass.

Consulting. We belong to Native Plant Society of $B C$. From our Zone 5 location Bluestem Nursery offers field-grown plants as well as flats of plugs, which are an inexpensive way to plant grasses in masses. Willows are an exciting new plant group. We encourage you to consider these plants, which you probably think of as only being represented by the giant weeping willow. There are many more that are suitable for even small gardens. Lots of willows have great winter color too. We sell these as cuttings and as plugs. Green roofs are a

favorite subject of ours. We are taking inquiries regarding plants for this relatively new concept in roofing. We are also testing different species for their suitability, particularly in cold locations. Our website provides a wealth of information on the subject of grasses and willows and how to successfully grow and use them in the landscape. There are numerous pictures to accompany the information. Don't miss our Grass Comparison Chart! Visit Bluestem Nursery's website: http://www.bluestem.ca.

Bluestem Prairie Nursery 13197 E 13th Rd, Hillsboro IL 62049 Contact Ken Schaal

Ph 217.532.6344

E-mail bluestemnursery@yahoo.com Private, retail, mail, since $1985.100 \%$ native, $100 \%$ propagated. Plants, Seeds.

Bluestone Perennials Inc

7211 Middle Ridge Rd,

Madison $\mathrm{OH} 44057$

Contact William (Bill) Boonstra

Ph 800.852.5243 Fax 440.428.7198

E-mail service@bluestoneperennials.com Private, wholesale, retail. Plants.

Bluff Creek Farm

PO Box 679, Hinsdale IL 60522

45 W 699 Jeter Rd, Big Rock IL 60511

Contact Bob Eileis

Ph 630.556.4808 Fax 630.556.4900

E-mail bob@bluffcreekfarm.com

Private, wholesale. Seeds.

Boehm's Garden Center

708 Macomb Rd, Rushville IL 62681

Contact Don Boehm

Ph 217.322.6644 Fax 217.322.2144

E-mail boehmsgc@frontiernet.net

Private, retail, M-F 8-4 Sat 8-12, since 1978. Plants.

Boething Treeland Farms Inc 2923 Alpine Rd,

Portola Valley CA 94028

Contact Constance Cook

Ph 650.851.4770 Fax 650.851.4252

Private, wholesale. Plants.

The Bosch Nursery Inc

18874 Hwy 4, Jonesboro LA 71251

Ph 318.259.9484 Fax 318.259.9443

Wholesale. Plants.

Bosch's Countryview Nursery 10785 84th Ave, Allendale MI 49401

Contact Brian Bosch

Ph 616.892.4090 Fax 616.892.4290

E-mail brian@boschsnursery.com

Private, wholesale. Plants, Seeds. 
Bosky Dell Natives

23311 SW Bosky Dell Ln,

West Linn OR 97068

Contact Lory Duralia

Ph 503.638.5945 Fax 503.638.8047

E-mail boskydellnatives@aol.com

Private, wholesale, retail, mail. Plants.

\section{BOTANICS WHOLESALE INC}

31701 SW 194th Ave,

Homestead FL 33030

Contact Mike Tevelonis or

Chris Oppenheimer

Ph 305.245.2966 Fax 305.246.1782

E-mail botanics@botanics.com

URL http://www.botanics.com

Private, wholesale. Plants.

For more than 25 years, we have supplied the industry with top-quality plants and unparalleled service. We offer more than 125 species of palms, cycads, native and flowering trees.

Botanique

387 Pitcher Plant Ln,

Stanardsville VA 22973

Contact Rob Sacilotto

E-mail botanique@pitcherplant.com

Private, wholesale, retail, mail. Plants.

Bow Point Nursery Ltd

244034 Range Rd 32,

Calgary AB T3Z 2E3

Contact Ken Wright

Ph 403.686.4434 Fax 403.242.8018

E-mail bowpoint@telus.net

Private, wholesale, retail. Plants.

\section{BOWMAN WILDFLOWER}

\section{PRESERVE}

PO Box 685, 1635 River Rd,

New Hope PA 18938

Contact Bill Lamack

Ph 215.862.2924 Fax 215.862.1846

E-mail lamack@bhwp.org or

bhwp@bhwp.org

Private, retail, M-F 9-5, since 1950. Plants:

$100 \%$ native, $100 \%$ propagated. Seeds:

$100 \%$ native, $100 \%$ propagated. Min:

cash/none, credit card $/ \$ 10$.

Premier destination to see nearly 1000 species of Pennsylvania native plants on 134 acres;

seeds by mail and on-site; plants on-site only.
Bowood Farms Inc

29319 Pike 235, Clarksville MO 63336

Contact John McPheeters

Ph 573.242.3840 Fax 573.242.3871

E-mail clarksville@bowoodfarms.com

Private, retail. Plants.

Boxelder Creek Nurseries

23809 Weld Co Rd 2,

Hudson CO 80642

Contact Dave Zach

Ph 303.279.8204 Fax 303.278.1832

E-mail boxelder@becn.us

Private, wholesale. Plants.

Boyd \& Boyd Nursery

7960 Smithville Hwy, Hwy 56 N,

McMinnville TN 37110

Contact Tom Boyd

Ph 931.934.2613 Fax 931.934.2044

E-mail info@boydandboydnursery.com

Private, wholesale. Plants.

Boynton Botanicals

9281 87th Place $S$,

Boynton Beach FL 33437

Contact Kathleen Kastenholz

Ph 800.642.1033; 561.737.1520

Fax 561.738.9598

E-mailkboynbot@aol.com

Private, wholesale. Plants.

Brandywine Conservancy

PO Box 141, US Rt 1 \& Creek Rd,

Chadds Ford PA 19317

Contact Mark Gormel

Ph 610.388.2700 Fax 610.388.3814

E-mail inquiries@brandywine.org

Private, retail. Plants, Seeds.

Breezy Oaks Nursery

23602 SE Hawthorne Rd,

Hawthorne FL 32640

Contact Larry Alsobrook

Ph 352.481.3795

Private, wholesale, retail. Plants.

Brent \& Becky's Bulbs

7900 Daffodil Ln, Gloucester VA 23061

Ph 804.693.3966 Fax 804.693.9436

E-mail

bbheath@brentandbeckysbulbs.com

Private, wholesale, retail, mail. Plants.
Brett Young

RR \#4, Corner of Hwy $39 \& 60$,

Calmar AB TOC OVO

Contact Gloria Weir

Ph 780.985.7314 Fax 780.985.3534

E-mail gloria.weir@brettyoung.ca

Private, wholesale, retail. Plants.

Briggs Nursery Inc

4407 Henderson Blvd, Olympia WA

98501

Ph 800.444.1515 Fax 206.352.5699

E-mail sales@briggsnursery.com

Private, wholesale. Plants.

Brokaw Nursery

PO Box 4818, Saticoy CA 93007

1419 Lirio Ave, Ventura CA 93004

Contact Rob Brokaw

Ph 805.647.2262 Fax 805.647.6493

E-mail rbrokaw@west.net

Private, wholesale. Plants.

Broken Arrow Nursery

13 Broken Arrow Rd, Hamden CT 06518

Contact Andrew Brand

Ph 203.288.1026 Fax 203.287.0135

E-mailinfo@brokenarrow.com

Private, wholesale, retail, mail, Internet. Plants.

Brooks Tree Farm

9785 Portland Rd NE, Brooks OR 97305

Contact Kathy Lecomptc

Ph 503.393.6300 Fax 503.393.0827

E-mail office@brookstreefarm.com

Private, wholesale. Plants.

BROWNING SEED INC

3101 South IH 27 Box 1,

Plainview TX 79072

Contact Rick Graves; John or Lee

Ph 806.293.5271 Fax 806.293.9050

E-mail orders@browningseed.com or rick@browningseed.com

URL http://www.browningseed.com

Private, wholesale, mail. Seeds.

Whether it's grain, vegetable, wildflowers, or various grass seeds, we have a broad range of products for your selection. Browning Seed has served the seed industry since 1962. Through the years, we have developed many new hybrids, including the first triple cross sorghumsudangrass hybrid known as Cadan 99B. Reclamation and restoration projects deserve the correct, clean, certified seed. If you're establishing a wildflower setting, check our website. You'll find seed for florals from the charming Johnny-jump-up, Indian blanket, and cornflower/bachelor's button to several arroyo 
lupine succulents, lemon/purple horse mint, moss verbena, and other treasures. Are you picturing acres and acres of gently swaying grass? Browning Seed has seed in quantity and special application grasses. Tour http://www.browningseed.com for ideas, prices, and maintenance products. We are your seed professionals.

See ad on page 159.

Buchanan's Native Plants

611 E 11th St, Houston TX 77008-7109

Contact Donna Buchanan

Ph 713.861.5702 Fax 713.861.2063

E-mail info@buchanansplants.com

Private, retail. Plants.

Buckeye Nursery Inc

PO Box 450, Perry FL 32348

1490 Buckeye Nursery Rd,

Perry FL 32347

Contact Johnny Brown

Ph 800.838.2218; 850.838.2680

Fax 850.838.2681

E-mail buckeyenursery@gtcom.net

Private, wholesale. Plants.

\section{BUFFALO-BERRY FARM}

51 E Lake Fork Rd, McCall ID 83638

Contact Jim Crawford or Margo Conitz

Ph 208.634.3062 Fax 208.634.3062

E-mail buffaloberryfarm@frontiernet.net URL http://www.buffaloberryfarm.com Private, wholesale, retail, mail, contract, 9-4:30 seasonally, since 1994 . Plants: $80,000 / y, 100 \%$ native, $100 \%$ propagated; container; tree, shrub, herbaceous, grass, wetland/aquatic. Seeds: tree, shrub, grass.

Experienced propagation and growing of plants native to the Intermountain Northwest.

Bundy CanyonTurf Supply 21420 Bundy Canyon Rd, Lake Wildomar CA 92595

Contact Doug Washburn

Ph 951.674.0638

E-mail agronotec@aol.com

Private, wholesale, retail, M-F 8-4, since 1984. Seeds.

Burgaw Creek Nursery \&

Turf Farm LLC

PO Box 1337, 1225 Stag Park Rd,

Burgaw NC 28425

Contact Dave Howard

Ph 910.259.4788 Fax 910.259.7039

E-mail burcreeknsyturf@intrstar.net

Private, wholesale. Plants.
Burnt Ridge Nursery

432 Burnt Ridge Rd,

Onalaska WA 98570

Contact Michael Dolan

Ph 360.985.2873 Fax 360.985.0882

E-mail mail@burntridgenursery.com

Private, wholesale, retail, mail, Internet.

Plants.

Busse Gardens

17160 245th Ave NW,

Big Lake MN 55309

Contact Alex Stolitza

Ph 763.263.3403 Fax 763.263.1013

E-mail customer.service@bussegardens. com

Private, wholesale, mail. Plants.

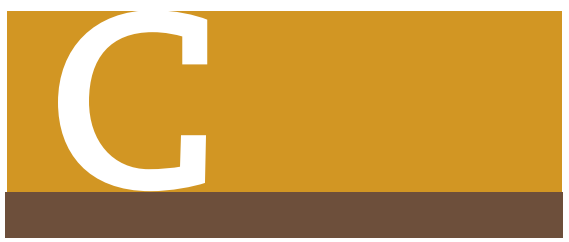

Cactus Joe's Blue Diamond

Cactus Nursery

PO Box 268, 12740 Blue Diamond Rd,

Blue Diamond NV 89004

Contact Tommy Lee

Ph 702.875.1968

E-mail shinrilee@gmail.com

State, retail. Plants.

Cal-Forest Nurseries

PO Box 719, 1838 Eastside Rd,

Etna CA 96027

Contact Tom Jopson

Ph 530.467.5211 Fax 530.467.5733

E-mail tomj@growpro.inc.com

Private, contract, M-F 8-5, since 1978.

Plants.

Calaveras Nursery

1622 Hwy 12, Valley Springs CA 95252

Contact Pat

Ph 209.772.1823 Fax 209.772.0864

Private, wholesale, retail, contract. Plants.

California Conservation Corps-

Napa Native Plant Nursery

PO Box 7199, Napa CA 94558

Ph 707.253.7783 Fax 707.253.1421

State, wholesale, contract. Plants.
California Dept of Forestry and

Fire Protection-Magalia

Reforestation Center

664 Steiffer Rd, Magalia CA 95954

Ph 530.873.0400 Fax 530.873.1473

Wholesale. Seeds.

California Dept of Forestry and Fire Protection-LA Moran

PO Box 1590, Davis CA 95617

Ph 530.753.2441 Fax 530.323.3401

E-mail laurie_lippitt@fire.ca.gov

State, wholesale. Seeds.

California Flora Nursery

PO Box 3, 2990 Somers St,

Fulton CA 95439

Contact Phil Van Soelen or

Sherrie Althouse

Ph 707.528.8813 Fax 707.528.1836

Private, wholesale, retail. Plants.

Callahan Seed

PO Box 5531, 6045 Foley Ln, Central

Point OR 97502

Contact Frank Callahan

Ph 541.855.1164 Fax 541.855.1164

E-mail callahanseeds@gmail.com

Private, wholesale, retail. Seeds.

Cambell Timberland

Management

2027 Kennedy Rd, Jasper TX 75951

Contact Tim Stewart

Ph 409.384.6164 Fax 409.384.9028

Private, wholesale. Plants.

Campbell Tree \& Land

Company Inc

PO Box 780, Wautoma WI 54982

Ph 920.787.4653 Fax 920.787.3696

E-mail campbellco@voyager.net

Private, wholesale. Plants.

Canfield Native Nursery

1369 Tilton Rd, Sebastopol CA 95472

Contact Patricia Canfield

Ph 707.823.3776 Fax 707.823.3776

E-mail patriciaanncanfield@comcast.net Private, wholesale, retail, by appt please call, since 1980. Plants: 30,000/y, 100\% native, $100 \%$ propagated; container; tree, shrub, herbaceous, grass, wetland/aquatic.

Contract growing, specializing in species from northern $C A$. 
Capitol Wholesale Nursery Inc

2938 Everdale Dr, San Jose CA 95148

Ph 408.239.0589 Fax 408.239.0357

E-mail cwnsales@sbcglobal.net

Private, wholesale, retail. Plants.

\section{Caprice Farm Nursery}

10944 Mill Creek Rd SE, Aumsville OR 97325

Contact Cyndi or Charlie Ph 503.749.1397 Fax 503.749.4097

E-mail cyndicap@wvi.com

Private. Plants.

Caras Nursery \& Landscape

2727 S 3rd St, Missoula MT 59804

Contact Helen Steiger

Ph 406.543.3333 Fax 406.543.2839 E-mail caras@preznan.net Private, retail. Plants.

Carencia Native Nursery

16101 Carencia Ln, Odessa FL 33556

Contact Rose Nimphius Ph 813.920.2737 Fax 813.920.7661

E-mail alicia@carencianursery.com

Private, wholesale. Plants.

Carino Nurseries

PO Box 538, Indiana PA 15701

857 Cameron Bottom Rd, Penn Run PA 15765

Contact Laura Carino

Ph 724.463.3350; 800.223.7075 Fax 724.463.3050

E-mail carino@carinonurseries.com

Private, wholesale. Plants.

Carlson Prairie Seed Farm Inc

2077 360th Ave, Lake Bronson MN 56734

Contact Mike Ratzlaff

Ph 877.733.3087; 218.689.4342 Fax 218.754.2694

E-mailmratzlaff@wiktel.com

Private, wholesale, retail. Seeds.

See ad on page 159.

Carmel Valley Seed Company

21 W Alaisal St, Salinas CA 93901

Contact Joel Panzer Ph 831.771.2557 Fax 831.771.2714

E-mailmwruck@sbcglobal.net

Private, wholesale, M-F 9-5. Seeds: $60 \%$ native.

Carmel Valley Seed Company provides native wildflowers and native grass seeds to wholesale and retail customers. Erosion control mixes are a major element of our business. We also offer custom wildflower seed packets for fund-raising or advertising.

\section{Carolina Greenery}

375 Carthage Rd, West End NC 27376

Ph 910.947.3150 Fax 910.947.3150

Private, wholesale, retail, mail. Plants.

\section{Specializing in CRP - Native Grasses • Reclamation Need ia? We have it, ar we'll find it? Browaing Seed, lne.}
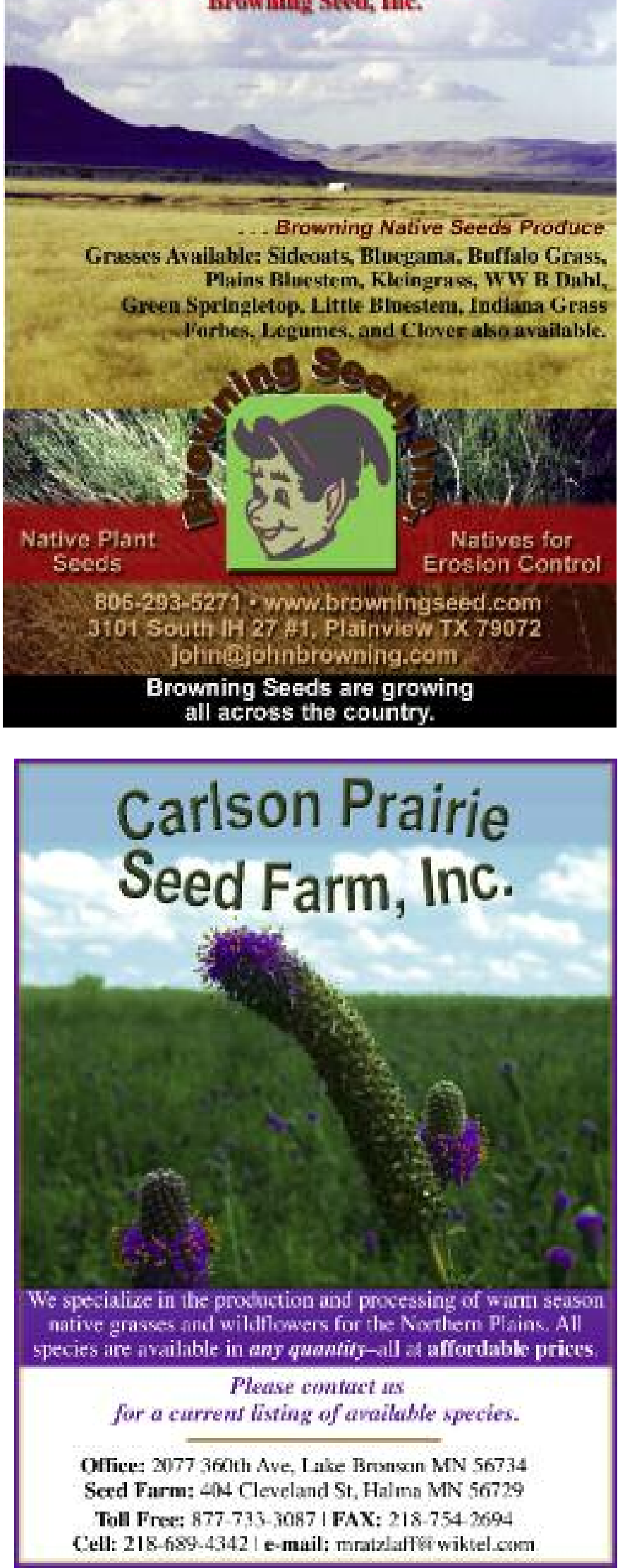
Carolina Native Nursery

1126 Prices Creek Rd, Burnsville NC

28714

Contact Bill Jones Ph 828.682.1471 Fax

828.682.1303

E-mail info@carolinanativenursery.com

Private, wholesale, retail. Plants.

Carolina Wild

314 Camellia Dr, Anderson SC 29625

Contact Christina Bruner

Ph 864.261.0659

E-mail nativeplants@carolinawild.com

Private, wholesale, retail. Plants.

Carter's Greenhouse \&

Nursery Inc

5145 Waymire Rd, Dallas OR 97338

Contact Steve Carter

Ph 503.787.3371 Fax 503.787.1502

E-mail cartersnursery@qwestoffice.net

Private, wholesale. Plants.

Carters Nursery-Bowater Forest

Products Division

11306 Hwy 411 S,

Chatsworth GA 30705

Ph 706.445.2422 Fax 706.445.4212

E-mail muellercw@bowater.com

Private, wholesale. Plants.

Casa Flora Inc

PO Box 41140, 5543 McCommas Bluff

Rd, Dallas TX 75241

Contact Rudy Nogeura

Ph 972.225.6111 Fax 972.225.5210

E-mail fernguys@casaflora.com

Private, wholesale. Plants.

Cascade Biomes

PO Box 22419, Seattle WA 98122-0419

Contact Virgie Newlin

Ph 206.322.0528 Fax 206.322.0528

E-mail biomes@earthlink.net

Private, retail. Plants, Seeds.

\section{CASCADE FORESTRY NURSERY}

1621 McCabe Ln, Cascade IA 52033

Contact Ed Schultz or Laura Bishop

Ph 800.596.9437 Fax 563.852.5004

E-mail cascade@cascadeforestry.com

URL http://www.cascadeforestry.com

Private, wholesale, retail, M-F 8-4:20 Sat

$8-12$, since 1975 . Plants: $95 \%$ native, $6 \%$ propagated, $60 \%$ wild collected. Seeds.

Northern hardy native hardwood/shrub low bed density produces heavily rooted vigorous seedlings. Also tree planting, plantation maintenance, timber consulting.

Cassidy Farm Nursery

PO Box 287, Cleveland NM 87107

Contact Dan Cassidy

E-mail dancas@nnmt.net

Private, wholesale, retail. Plants.

Catalina Island Conservancy-

James H Ackerman Native

Plant Nursery

PO Box 2739, 125 Clarrisa Ave,

Avalon CA 90704

Contact Peter Dixon

Ph 310.510.2904

E-mail pdixon@catalinaconservancy.org

Nonprofit, wholesale. Plants.

Catskill Native Nursery

607 Samsonville Rd,

Kerhonkson NY 12446

Contact Francis Groeters

Ph 845.626.2758 Fax 845.626.2758

E-mailinfo@catskillnativenursery.com

Private, retail. Plants.

Cavicchio Nurseries

110 Codjer Ln, Sudbury MA 01776

Contact Lizza Mets

Ph 978.443.7177 Fax 978.639.6316

E-mail lizza@cavicchio.com

Private, wholesale. Plants.

Cedera Seed Inc

PO Box 97, 118 State Hwy 31,

Swan Valley ID 83449

Contact Delbert or June

Ph 208.483.3683; 208.423.3684

E-mail delbert684@cs.com

Private, wholesale, retail, since 2000. Seeds.

The Center for Social and

Environmental Stewardship

(formerly Circuit Rider

Productions Inc)

9619 Old Redwood Hwy,

Windsor CA 95492

Contact Anya Perron-Burdick

Ph 707.838.6641 x231

Fax 707.838.4503

E-mail aburdick@cfses.org

Nonprofit, wholesale, retail, M-F by appt,

since 1976. Plants: $50,000 / y, 100 \%$ native,

$100 \%$ wild collected; container.

Nonprofit community organization.
Central Coast Wilds

125 Walk Circle, 336A Golf Club Dr, Santa Cruz CA 95060

Contact Ellen Holmes

Ph 831.459.0656 Fax 831.457.1606

E-mail ellen@centralcoastwilds.com

Private, wholesale, contract. Plants, Seeds.

See ad on page 161.

Central Florida Lands and Timber

3087 N Co Rd 53, Mayo FL 32066

Contact Tony Barrington

Ph 386.294.1211 Fax 386.294.3416

E-mail cflat@windstream.net

Private, wholesale. Plants.

Central Utah Seed

$825 \mathrm{~N} 400$ E, Ephraim UT 84627

Ph 435.283.4344 Fax 435.283.4344

Private, wholesale, retail. Seeds.

CHAMPOEG NURSERY INC

9661 Yergen Rd NE, Aurora OR 97002

Contact Paul M Stormo

Ph 503.678.6348 Fax 503.678.4348

E-mailinfo@champoegnursery.com

URL http://www.champoegnursery.com

Private, wholesale, mail, contract, M-F 8-5,

since 2002. Plants: $300,000 / y, 100 \%$ native,

$100 \%$ propagated; container, bareroot,

$B \& B$, cuttings; tree, shrub, herbaceous,

grass, wetland/aquatic. Seeds: $100 \%$ native,

$100 \%$ wild collected; tree, shrub, herba-

ceous, grass. Min: $\$ 100$.

Specializing in native plants of the Pacific and Inland Northwest.Champoeg Nursery Inc grows an extensive collection of plants that are native to the Pacific and Inland Northwest.

Our palette of plants includes a variety of trees, shrubs, herbaceous perennials, groundcovers, and wetland plants. Since we have been in business, our primary goal has been to provide our customers with consistently highquality plants and service. In doing this, we have had the opportunity to build long-lasting relationships with many public and private organizations, other nurseries, and landscapers. Our commitment to quality is the foundation of all of our working relationships, and we hope that we have the opportunity to work with you.

Charles Nii Nursery

908 Kamilonui PI, Honolulu HI 96825

Contact Glenn

Ph 808.395.9959 Fax 808.395.9959

E-mail cniihyhi@hawaii.rr.com

Private, wholesale, retail. Plants. 
Chas C Hart Seed Company

304 Main St, Wethersfield CT 06109

Contact Robert Hart

Ph 860.529.2537 Fax 860.563.7221

E-mail info@hartseed.com

Private, wholesale. Seeds.

Chelsea Nursery

3347 G Rd, Clifton CO 81520-8143

Ph 970.434.8434 Fax 970.523.0737

Private, retail. Plants.

Chesapeake Native Nursery

326 Boyd Ave \#2,

Takoma Park MD 20912

Contact Sara Tangren

Ph 301.580.6237

E-mail satangren@chesapeakenatives.com or

sales@chesapeakenatives.org

Private, wholesale, retail, M-F 9-5, since 2005. Plants, Seeds. Min: $\$ 100$.

\section{CHIAPPINI FARM NATIVE NURSERY}

PO Box 436, Melrose FL 32666

Contact David Chiappini

Ph 800.293.5413 Fax 352.475.5268

E-mail dchiapin@atlantic.net

Private, wholesale, $8-5$, since 1984 . Plants: $100 \%$ native, $100 \%$ propagated.

30-acre Florida native plant containerized nursery for North Florida and lower Southeast US. Most of our 170 species are available in large quantities. Contract growing is encouraged.

Chief River Nursery

10681 N McClain Rd, Hayward WI 54843

Ph 800.367.9254 Fax 866.226.5204

E-mail chiefriver@gmail.com

Private, wholesale. Plants.

Cicconi Farms Inc I The Perennial Specialists

1005 Farmingdale Rd, Jackson NJ 08527

Contact Albert Cicconi

Ph 732.363.1420 Fax 732.905.9242

E-mail al@cicconifarms.com

Private, wholesale. Plants.

Circle S Seeds of MT Inc

PO Box 130, 14990 Madison Frontage Rd,

Three Forks MT 59752

Contact John McDonald

Ph 406.285.3269 Fax 406.285.3040

Private, wholesale, retail. Seeds.

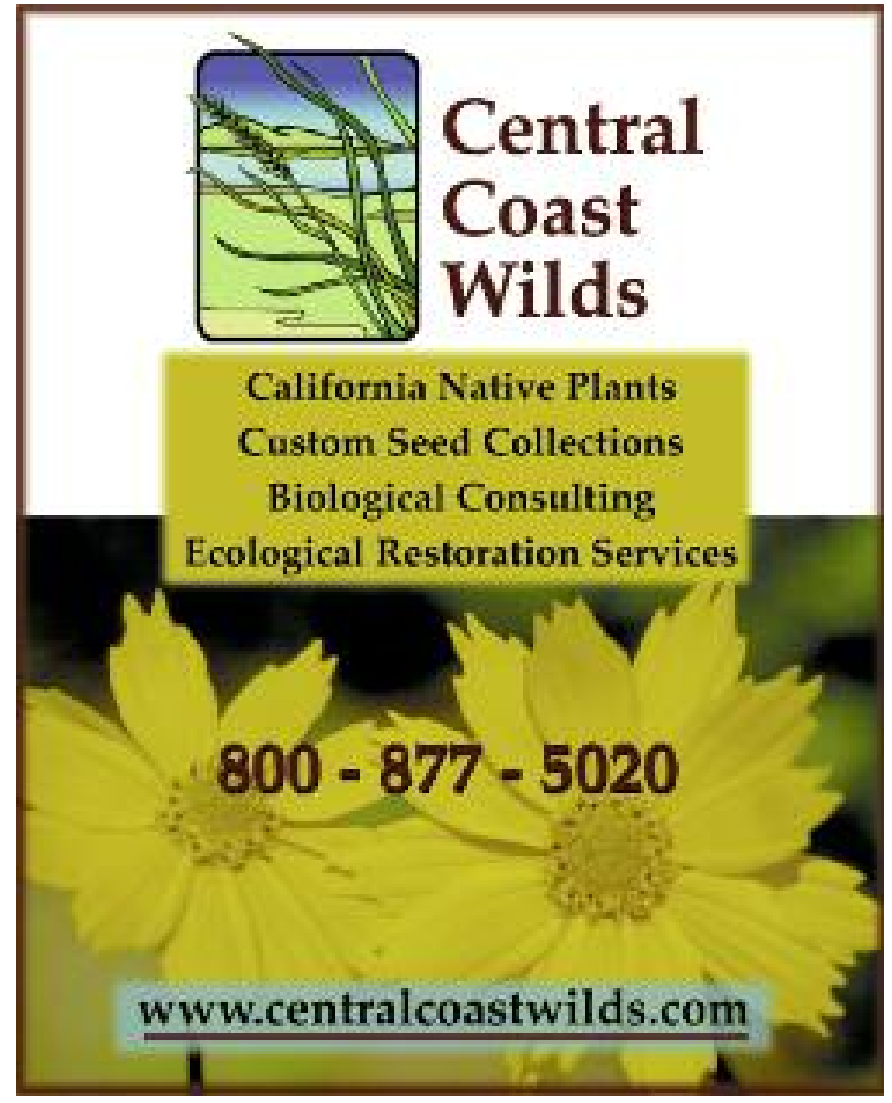

\section{directory key}


City of New York Parks and

Recreation-Greenbelt Native

Plant Center

3808 Victory Blvd,

Staten Island NY 10314

Contact Timothy Chambers

Ph 718.370.9044 ×304

Fax 718.370 .0932

E-mail gnpcinfo@parks.nyc.gov

Wholesale. Plants.

Clear Ridge Nursery Inc

217 Clear Ridge Rd, 3360 Lowman Ln, Union Bridge MD 21791

Contact Jessica Barley

Ph 410.775.7700 Fax 410.848.5806

E-mail crn@qis.net

Private, wholesale, retail. Plants.

Clearwater Greenhouses

PO Box 423, Big River SK SOJ OE0

Contact Gary McLean

Ph 306.469.2111 Fax 306.469.4439

E-mail clearwater@cwgh.ca

Private, wholesale. Plants.

Clearwater Native Nursery

1980 SW 55th St, Redmond OR 97756

Contact Mike

Ph 541.350.5261 Fax 541.504.5995

E-mail mike@clearwaternatives.com

Private, contract. Plants.

Clemenson Farms Native Nursery

PO Box 215, 108 Linwood Ave, Estell

Manor NJ 08319

Ph 609.476.3903 Fax 609.476.3903

Private, wholesale. Plants.

Clifton Forest \& Appraisal Service Inc

PO Box 882, 302 Donehoo St,

Statesboro GA 30458

Contact Jeremy Coulter

Ph 912.489.8250 Fax 912.489.2066

Services.

Clifton-Choctaw Nursery

1146 Clifton Rd, Clifton LA 77447

Contact Watson Clifton

Ph 318.793.4253 Fax 318.793.4211

E-mail ccri@bellsouth.net

Tribal, wholesale. Plants.
Clifty View Nursery

312 Clifty View Rd,

Bonners Ferry ID 83805

Contact Steve Koppang

Ph 208.267.7129 Fax 208.267.8559

E-mail stevek@cliftyview.com

Private, wholesale, mail, contract. Seeds.

Cloud Mountain Farm

6906 Goodwin Rd, Everson WA 98247

Contact Terry Macziga

Ph 360.966.5859 Fax 360.966.0921

E-mailinfo@cloudmountainfarm.com

Private, retail, mail, Internet, M-Sat 10-5

Sun 11-4 Feb-Jun or by appt, since 1979 .

Plants: $15 \%$ native, $100 \%$ propagated; con-

tainer, bareroot, $B \& B$, cuttings.

Clyde Robin Seed Company Inc

PO Box 411, Santa Clara UT 84765

1120 Tewepea Ln, Ivans UT 84738

Contact Steve Atwood

Ph 510.315.6720 Fax 435.216.5414

E-mail sales@clyderobin.com

Private, retail, M-F 8-5, since 1935. Seeds:

herbaceous.

CNPS Inc

5951 Olgesby Rd, Milton FL 32570

Contact John

Ph 850.623.6287 Fax 850.626.2684

E-mail cnpsair@aol.com

Private, wholesale, retail. Plants.

Coastal Gardens \& Nursery

4611 Socastee Blvd,

Myrtle Beach SC 29588

Ph 843.293.2000 Fax 843.293.4448

E-mail gardens1@sccoast.net

Private, retail, mail. Plants.

Coastal Native Plants Nursery LLC

PO Box 42, 9698 Highland St,

Mauricetown NJ 08239

Contact Bryan Alcox

Ph 856.785.1102 Fax 856.785.1102

E-mail bryanalcox@verizon.net

Private, wholesale. Plants.

Coastal Plain Conservation

Nursery

550 E Westinghouse Blvd,

Charlotte NC 28273

Contact Gregg Antemann

Ph 704.527.1177 Fax 704.527.1133

E-mail gregg@cws-inc.net

Private, wholesale, retail. Plants.
COASTAL PLAIN CONSERVATION

NURSERY INC

3067 Conners Dr, 812 Drummonds

Point Rd, Edenton NC 27932

Contact Ellen J Colodney PhD or Jean Bridges

Ph 252.482.5707 Fax 252.482.4987

E-mail sales@coastalplainnursery.com URL http://www.coastalplainnursery.com

Private, wholesale, by appt, since 1999.

Plants: $100 \%$ native, $100 \%$ propagated.

Coastal Plain Conservation Nursery Inc propagates more than 100 woody and herbaceous species for ecosystem restoration projects in the Carolinas and Tidewater Virginia.

Cold Stream Farm

2030 Free Soil Rd, 8585 Stephens Rd,

Free Soil MI 49411

Contact Mike Hradel

Ph 231.464.5809 Fax 231.464.5809

E-mail info@coldstreamfarm.net

Private, wholesale, retail, mail, M-Sat $8-5$ by appt, since 1978. Plants: 500,000/y, 80\% native, $98 \%$ propagated, $2 \%$ wild collected; bareroot, cuttings, bioengineering.

Collector's Nursery

16804 NE 102nd Ave,

Battle Ground WA 98604

Contact Diana

Ph 360.574.3832 Fax 360.574.3596

E-mail dianar@collectorsnursery.com

Private, retail, mail. Plants.

Colorado Seed Solutions

PO Box 68, 195 N Washington,

Monte Vista CO 81144

Contact Terry Hillin

Ph 719.852.3505 Fax 719.852.4942

E-mail thillin@seedsolutions.com

Private, wholesale, retail, mail. Seeds.

\section{COLORADO STATE FOREST}

\section{SERVICE NURSERY}

Colorado State University,

5060 Campus Delivery,

Fort Collins CO 80523-5060

3843 W La Porte Ave,

Fort Collins CO 80523

Contact Randy Moench

Ph 970.491.8429 Fax 970.491.8250

E-mail treeseedlings@hotmail.com

URL http://www.csfs.colostate.edu/

nursery.htm

State, retail, mail, contract, 7:30-4, since 1957. Plants: 2.5 million/y, 65\% native, $100 \%$ propagated; container, bareroot, cuttings; tree, shrub. 
Producer of quality conservation seedlings for more than $50 y$. Both greenhouse grown and bareroot.

Colville Tribal Forestry

Greenhouse

PO Box 72, Nespelem WA 99155

Contact Diana Seymour

Ph 509.634.2896 Fax 509.634.8685

Tribal. Plants, Seeds.

\section{Colvos Creek Nursery}

20211 Vashon Hwy SW,

Vashon WA 98070

Contact Mike Lee

Ph 206.749.9508 Fax 206.749.0446

E-mailcolvos@gmail.com

Private, wholesale, retail, mail. Plants.

COMPANION PLANTS INC

7247 N Coolville Ridge,

Athens OH 45701

Contact Peter Borchard

Ph 740.592.4643 Fax 740.593.3092

E-mail complants@frognet.net or info@companionplants.com

URL http://www.companionplants.com

Private, wholesale, retail, mail, Internet,

Th-Sun 10-5, since 1982. Plants: 40,000/y,

$30 \%$ native, $90 \%$ propagated, $10 \%$ wild collected; container, bareroot; shrub, herba-

ceous, wetland/aquatic. Seeds: varies/y, 30\% native, $75 \%$ propagated, $25 \%$ wild collect-

ed; shrub, herbaceous.

Producer of quality conservation seedlings for more than 50 years. Both greenhouse grown and bareroot. More than 600 varieties of common and exotic herb plants and seeds. Useful plants from our backyard and around the world.

\section{Comstock Seed}

917 Hwy 88, Gardenerville NV 89460

Contact Ed Kleiner

Ph 775.265.0090 Fax 775.265.0040

E-mail ed@comstockseed.com

Private, wholesale, retail, mail. Seeds.

Concepts in Greenery Inc

16366 Old Cheney Hwy,

Orlando FL 32833

Contact Chris Poe

Ph 407.568.2000 Fax 407.568.0522

E-mail office@conceptsingreenery.com

Private, wholesale. Plants.
Confederated Tribes of the

Umatilla Indian Reservation

PO Box 638, 73820 Hwy 331,

Pendleton OR 97801

Contact Ruth Whittaker

Ph 541.278.8525

E-mail tribalnativeplants@wtechlink.com

Tribal, wholesale, retail, contract. Plants.

Confreda Greenhouses \& Farms

2150 Scituate Ave, Hope RI 02831

Ph 401.827.5000 Fax 401.826.3276

Private. Plants.

\section{Conservation Seeding \&}

Restoration Inc

506 Center St W, Kimberly ID 83341

Contact James Ells

Ph 208.423.4835 Fax 208.423.4808

Private, wholesale. Plants, Seeds.

\section{CORNFLOWER FARMS}

PO Box 896, Elk Grove CA 95759

9811 Sheldon Rd, Elk Grove CA 95624

Contact Janet Gerland or Neal Funston

Ph 916.689.1015 Fax 916.689.1968

E-mail natives@cornflowerfarms.com

Private, wholesale, retail, M-F 7-7:30, since

1981. Plants: $90 \%$ native, $100 \%$ propagated.

Cornflower Farms is a leading grower of quality West Coast native plant material for wildland restoration and site revegetation. We grow more than 700 site-identified species and offer collection and contract growing services for site-specific projects.

See ad on page 165 .

The Cotton Mill I Star Nursery 385 W Telegraph Rd, Washington UT 84780

Ph 435.986.0820

Private, wholesale, retail. Plants.

Country Landscapes Inc 56985 Lincoln Hwy, Ames IA 50010 Contact Renee Hanlon

Ph 515.232.6864 Fax 515.232.8325

E-mail renee@countrylandscapes.com Private, retail. Plants.

\section{COUNTRY ROAD}

GREENHOUSES INC

19561 E Twombly, Rochelle IL 61068

Contact Larry Creekmur

Ph 815.384.3311

E-mail crginc@prairieplugs.com

URL http://www.prairieplugs.com

Private, wholesale, M-F 9-5, since 1983.

Plants: $100 \%$ native, $100 \%$ propagated;

herbaceous, grass, wetland/aquatic.

Midwest grasses, sedges, forbs, stream bank, and lake shore species. Heavily rooted Prairie

Plugs are 2.5 inches wide and 4.5 inches deep.

The Country Store \& Gardens 20211 Vashon Hwy SW, Vashon WA 98070

Ph 206.463.3655 Fax 206.463.3679

E-mail info@countrystoreandgardens.com

Private, wholesale, retail. Plants.

Countryside Trees

290 Meadow Glade,

San Antonio TX 78227

Ph 210.674.1693 Fax 210.674.1693

Private. Plants.

County of Los Angeles Fire Dept

Forestry Division, 5823 Rickenbacker Rd Room 123, Commerce CA 90040-3027

Contact Bill Romo, Deputy Forester

Ph 323.890.4330 Fax 323.890.4335

E-mail forestry_fb@lacofd.org

State, wholesale. Seeds.

Cousin's Nursery

5144 36th Ave SE, Salem OR 97301

Contact Chuck Mangum

Ph 503.364.1240 Fax 503.364.3246

E-mail cmacousn@msn.com

Private, wholesale. Plants.

Coyote Creek Inc

9382 Island Rd, St Francisville LA 70775

Contact Tina Reid

Ph 225.635.6736; 866.562.8088

Fax 225.635.3647

E-mail coyotecreek@att.net

Private, retail, mail. Plants.

Creekside Gardens

PO Box 357, Miranda CA 95553

Contact Consuelo Evans

Ph 707.367.1961

E-mail creeksideg@aol.com

Private, wholesale. Plants. 
Cricket Hill Garden

670 Walnut Hill Rd,

Thomaston CT 06787

Contact David or Kasha Furman

Ph 186.028.3104 x2 Fax 860.283.5508

E-mail kasha@treepeony.com

Private, wholesale, retail. Plants.

Croshaw Nursery

PO Box 339, 113 Mill Ln,

Columbus NJ 08022

Contact David Croshaw

Ph 609.298.0477 Fax 609.298.6388

E-mail croshawnursery@mac.com

Private, wholesale. Seeds.

CS\&KT Forestry Tribal Nursery

104 Main St SE, Ronan MT 59864

Contact Jean Matt

Ph 406.676.3755 x6032

Fax 406.676.3756

E-mail info@cskt.org

Tribal, retail. Plants.

Cure Nursery

880 Buteo Ridge Rd,

Pittsboro NC 27312-9332

Contact Bill or Jennifer Cure

Ph 919.542.6186 Fax 919.542.6186

E-mail curenursery@mindspring.com

Private, wholesale, by appt please call, since 1995. Plants.

Cure Nursery specializes in propagation and wholesale of woody native plants in small containers for the regional restoration/landscape trade. See our website for current offerings.

Curry Native Plants

92545 Silver Butte Rd,

Port Orford OR 97465

Contact Dale Lee

Ph 541.332.5635

E-mail daleenews@hotmail.com

Private, wholesale. Plants.

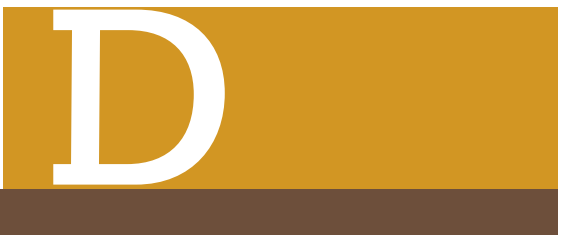

D Wells Farms

PO Box 336, Hubbard OR 97032

Contact Daniel

Ph 503.982.1012 Fax 503.981.8420

E-mail sales@dwellsfarms.com

Private, wholesale. Plants.
Darwin's Backyard Nursery

PO Box 3532, Cullowhee NC 28723

Contact Doyle Darwin Thomas

E-mail bugeaters4u@aol.com

Private, retail. Plants.

David R Mosman Ranch Inc

3160 Mosman Rd, Craigmont ID 83523

Ph 208.937.2552 Fax 208.937.2552

E-mailmosman@camasnet.com

Private, wholesale, retail. Plants.

DeepSouth Pine Nursery Inc

PO Box 267, 5550 Boomerang Rd,

Bascom AL 32423

Ph 888.839.2488

E-mail dpsofor@surfsouth.com

Private, wholesale. Plants.

DeLange Seed Inc

PO Box 7, 537 W Hwy 47,

Girard KS 66743

Contact Steve Ahring

Ph 800.962.5429 Fax 620.724.6222

E-mail steve@delangeseed.com or

delangeseed@ckt.net

Private, wholesale, retail, M-F 8-5 Sat 8-12, since 1970 . Seeds.

Delaware Native Plant Society

PO Box 369, Dover DE 19901

Contact Erric Zuelke

Ph 302.735.8918

E-mail ezuelke@juno.com

Private. Plants.

Delta-View Nursery

Rt 1 Box 28, 659 Burdette Rd, Leland

MS 38756

Ph 800.748.9018 Fax 662.686.2353

E-mail hardwoods@tecinfo.com

Private, wholesale. Plants.

Deluxe Trees and Shrubs

6306 SW Carlton Ave,

Arcadia FL 34266

Contact Charles or Darlene Foster

Ph 863.494.1488 Fax 863.993.9369

E-mail deluxe@strato.net

Private, wholesale. Plants.
DERBY CANYON NATIVES

PO Box 385, 9750 Derby Canyon Rd, Peshastin WA 98847

Ph 509.548.9404 Fax 509.548.9404

Contact Ted Alway

E-mail ted@derbycanyonnatives.com

Private, wholesale, retail, contract. Plants, Seeds.

We grow more than 120 species of forbs, grasses, shrubs, and trees native to Central Washington. Container-grown plants are produced from seeds and cuttings from the area's diverse habitats, including the shrub-steppe, forest, and riparian zones. Plants are sourceidentified and locally adapted. Custom growing, seed, and cutting collection available.

Desert Enterprises

PO Box 23, 25202 W Rockaway Hills, Morristown AZ 85342

Contact Judith Clement

Ph 623.388.2448 Fax 623.388.9805

E-mail desertent@aol.com

Private, wholesale, retail. Plants.

Desert Jewels Nursery

9809 E Upriver Dr, Spokane WA 99206 Ph 509.893.3771

E-mailinfo@desertjewelsnursery.com Private, retail, contract. Plants.

Desert Land Nursery

11306 Gateway E, El Paso TX 79927

Contact Sarah M Guerra-Brown

Ph 915.858.1130

E-mail desertland@att.net

Private, wholesale, retail, M-Sat 8-5 Sun 10-5 spring/summer; M-Sun 10-4 fall/winter, since 1970. Plants, Seeds.

Desert Survivors

1020 W Starr Pass, Tucson AZ 85713

Contact Jim Verrier

Ph 520.884.8806 ×21; 520.791.9309

Fax 520.884.0940

E-mail jim@desertsurvivors.org

Private, retail. Plants.

Desert Trees Nursery

9559 N Camino Del Plata,

Tucson AZ 85741

Contact Roger Young

Ph 800.873.3041; 520.297.5664

Fax 520.297.5035

E-mailmfontez47@aol.com

Private, wholesale. Plants. 
Detlor Tree Farm

Box 6, Plainfield WI 54966

Ph 715.335.4444 Fax 715.335.4442

E-mail detcoinc@hotmail.com

Private, wholesale. Plants.

Dieter Martin Greenhouse Ltd

Box 84, Langham SK SOK 2L0

Contact Dieter Martin

Ph 306.283.4376 Fax 306.283.4908

Private, wholesale, retail. Plants.

\section{Digging Dog Nursery}

PO Box 471, 31101 Middle Ridge Rd, Albion CA 95410

Contact Lynn Chrysler or Joanne Jewell

Ph 707.937.1130 Fax 707.937.2480

Private, mail. Plants.

Diversity Farms

25494 320th, Dedham IA 51440

Ph 712.683.5555 Fax 712.683.5535

E-mail dfarms@pionet.net

Private, wholesale, retail. Plants.

Doak Creek Native Plant Nursery

83331 Jackson Marlow Rd, Eugene OR 97405

Contact Cynthia Lafferty

Ph 541.484.9206 Fax 541.484.7358

Private, wholesale, retail. Plants.

Dodd \& Dodd Native Nurseries

PO Box 439, 9585 Wulff Rd, Semmes AL 36575

Contact Tom or Thayer Dodd

Ph 251.645.2222 Fax 251.645.2222

E-mailinfo@doddnatives.com

Private, wholesale. Plants.

Dodds Family Tree Nursery

515 W Main St, Fredericksburg TX 78624

Contact John Dodd Ph 830.997.9571

E-mail dodds@beecreak.net or info@dodds-nursery-florist.com

Private, wholesale, retail, since 1977. Plants.

Donaroma's Nursery

PO Box 2189, 271 Upper Main St, Edgartown MA 02539

Contact Michael Donaroma

Ph 508.627.8366; 508.627.8595 Fax 508.627.7855

E-mail donaromas@donaromas.com

Private, retail. Plants.

Doremus Wholesale Nursery

2167 Co Rd 1550, Warren TX 77664

Contact Mark or Ted

Ph 409.547.3536 Fax 409.547.3204

E-mail edoremus3@aol.com

Private, wholesale. Plants.

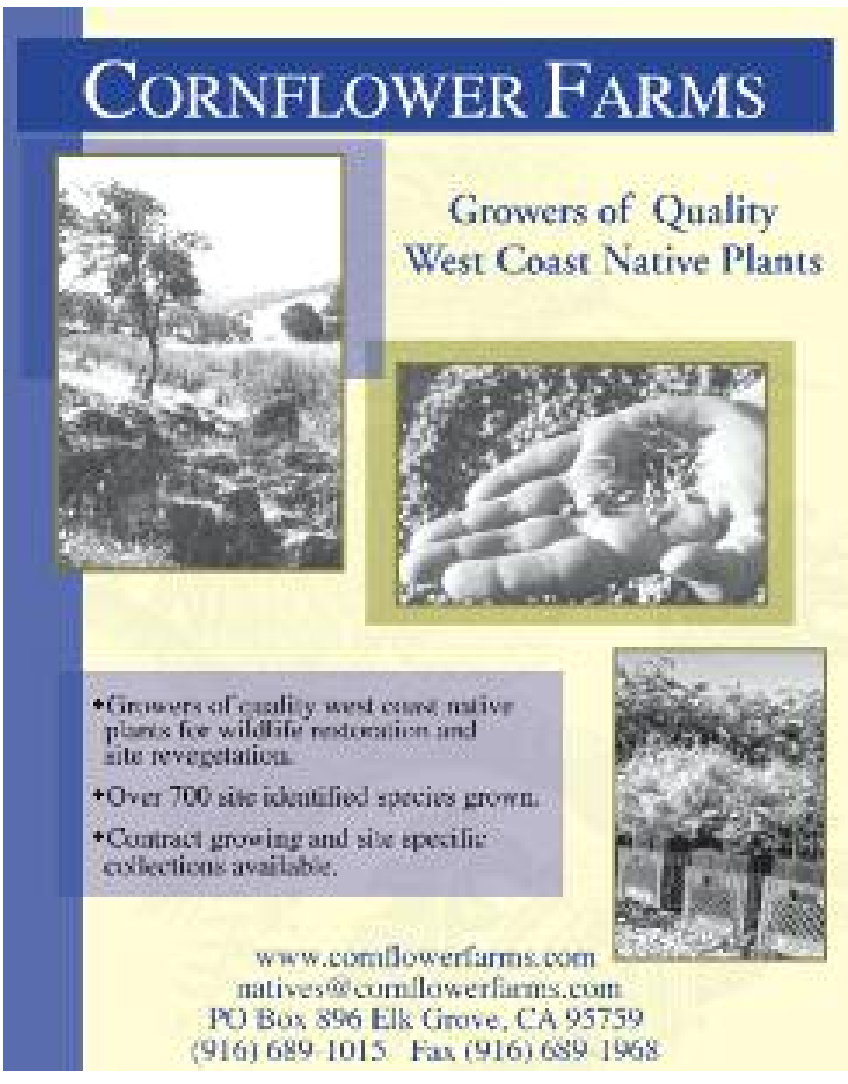

Douglass King Company Inc

PO Box 200320, San Antonio TX 78220

Contact Blayne

Ph 210.661.4191 Fax 210.661.8972

E-mailinfo@dkseeds.com

Private, wholesale, retail. Seeds.

Doyle Farm Nursery

158 Norris Rd, Delta PA 17314

Contact Jacquelin L Doyle

Ph 717.862.3134 Fax 717.862.3134

E-mail jld@doylefarm.com

Private, mail, Internet. Plants, Seeds.

\section{DR Bates}

PO Box 68, Loxahatchee FL 33470

Contact DR Bates

Ph 561.790.3246 Fax 561.795.3366

E-mail drbates@earthlink.net

Private, wholesale, retail. Plants.

Draggin' Wing Farm, Water-Thrifty

Plants for Idaho

5218 Castle Dr, Boise ID 83703

Contact Diane

Ph 208.345.4199

E-mail diane@waterthriftyplants.com

Private, wholesale, retail. Plants. 
Drakes Crossing Nursery 19774 Grade Rd SE, Silverton OR 97381

Ph 503.873.4932 Fax 503.873.4933

E-mail info@drakescrossingnursery.com Private, wholesale. Plants.

Dropseed Native Nursery

1205 S Buckeye Ln, Goshen KY 40026

Contact Margaret Shea

Ph 502.439.9033

E-mailmargaret@dropseednursery.com Private, retail, contract. Plants, Seeds.

Dry Valley Nurseries

667 Curtis Rd, Kelowna BC V1V 2C9

Contact Steve Duncan

Ph 250.860.6468 Fax 250.860.6836

E-mail dryvalleynurseries@telus.net

Private, retail. Plants.

Dry West Nursery LLC

PO Box 429, 32963 J Rd,

Hotchkiss CO 81419

Contact David Stiller

Ph 970.872.4172 Fax 970.872.4468

Private, wholesale, retail. Plants.

Duckwater-Shoshone Nursery

PO Box 140068, Duckwater NV 89314

Contact Kip Alexander

Ph 775.863.0299

Tribal, wholesale. Plants.

Durio Nursery

5853 Hwy 182, Opelousas LA 70570

Contact Dalton E Durio

Ph 337.948.3696 Fax 337.942.6404

E-mail dalton@durionursery.biz

Private, wholesale, retail. Plants.

Dwight Stansel Farm \& Nursery

5553 164th St, Wellburn FL 32094

Contact Carol Stalvey

Ph 386.963.2827 Fax 386.963.1440

E-mail dsfntte@windstream.net

Private, wholesale, retail. Plants, Seeds.

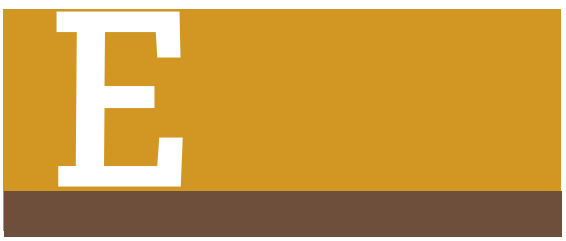

E Nakashima Greenhouses

PO Box 438, Honoka'a HI 96727

Contact Earl

E-mail ebnakashima@excite.com

Private, retail. Plants.

Eagle Lake Nurseries Ltd

PO Box 2340, $5 \mathrm{~km}$ east $2.5 \mathrm{~km}$ south

of Strathmore, Strathmore AB T1P 1K5

Contact Tony Heuver

Ph 403.934.3670 Fax 403.934.3626

E-mail jim@eaglelakenurseries.com

Private, wholesale. Plants.

Earth Tones Native Plant Nursery

212 Grassy Hill Rd,

Woodbury CT 06798

Contact Kyle or Lisa

Ph 203.263.6626 Fax 203.263.6626

Private, retail. Plants.

EarthBalance

2579 N Toledo Blade Blvd,

North Port FL 34289

Contact Barbie Divinetti

Ph 888.536.2855; 941.426 .7878

Fax 941.426 .8778

E-mail divinetti@earthbalance.com

Private, wholesale, contract, M-F 8-5, since 1985. Plants.

Earthly Goods Ltd

620 E Main St, New Albany IN 47150

Contact Ann Streckfus

Ph 812.944.3283 Fax 812.944.2903

Private, retail. Seeds.

Earthskin Nursery

9331 NCR 3800E, Mason City IL 62664

Contact Lou Nelms

Ph 217.482.3524; 217.737.6783

E-mail Irnelms@springnet1.com

Private, wholesale, retail. Seeds.
East Texas Seed Company

PO Box 569, Tyler TX 75710-0569

1030-D E Commerce, Tyler TX 75702

Contact Bill

Ph 800.888.1371; 903.597 .6637

Fax 903.595.0106

E-mail

seeds@easttexasseedcompany.com

Private, wholesale, retail. Seeds.

Eastern Sierra Native Plants

140 Willow Rd, Bishop CA 93514

Ph 760.387.2913 Fax 760.387.2961

E-mail ingram@telis.org

Private, wholesale, contract. Plants.

Eby Nursery Inc

PO Box 1127, Wilsonville OR 97070

Ph 503.638.8222 Fax 503.638.3848

E-mail bceby@teleport.com

Private, wholesale. Plants.

ECEC Native Plant Nursery

PO Box 684, 15850 Hwy W,

Elkton OR 97436

Ph 541.584.2692 Fax 541.584.2692

E-mail butterfly@cascadeaccess.com

Private, wholesale, retail, contract. Plants, Seeds.

The Echo Center

1055 Echo Circle, Pensacola FL 32514

Contact Ed or Perrin Penniman

Ph 850.478.1985

E-mail echocenter@yahoo.com

Private, retail. Plants.

Echo Nursery

6615 Co Rd 214,

Keystone Heights FL 32656

Contact Wes Tucker

Ph 352.475.1923 Fax 352.475.5529

Private, wholesale. Plants.

Echo Valley Natives

18883 S Ferguson Rd,

Oregon City OR 97045

Contact Elizabeth A Bluemmel

Ph 503.631.2451 Fax 503.631.2451

E-mail echovalleynatives@msn.com

Private, wholesale, retail. Plants. 
Eco Gardens

PO Box 1227, Decatur GA 30031

1346 S Indian Creek Dr, Stone

Mountain GA 30083-5251

Contact Don Jacobs

Ph 404.294.6468 Fax 404.294.8173

E-mail ecogardens@bellsouth.net

Private, retail, mail. Plants.

ECOLAGE Purveyors of Wonder 2623 W Sale Rd, Lake Charles LA 70605

Contact Stephen Abramans

Ph 337.562.2188; 866.562.8088

Fax 337.477.5992

E-mail ecolage@bellsouth.net

Private, retail. Plants.

Ecological Consultants Inc

5121 Ehrlich Rd, Ste 103A,

Tampa FL 33624

Contact Donald Richardson

Ph 813.264.5859 Fax 813.264.5957

E-mail fo.eci@verizon.net

Services.

Edge of the Prairie Wildflowers

1641 W Oak Hill Rd,

Crawfordsville IN 47933

$\mathrm{Ph} 765.362 .0915$

E-mail edgeoftheprairie@webtv.net

Private, wholesale, retail. Plants.

Edge of the Woods Native Plant

Nursery LLC

2415 Rt 100, Orefield PA 18069

Contact Louise Schaefer

Ph 610.395.2570 Fax 610.285.2471

E-mail info@edgeofthewoods.com

Private, wholesale, retail. Plants.

Edwards Greenhouse

4106 Sand Creek St,

Boise ID 83703

Contact Trisha

Ph 208.342.7548 Fax 208.342.0411

Private, retail. Plants, Seeds.

EL NATIVO GROWERS INC

200 S Peckham Rd, Azusa CA 91702

Contact James Campbell or

Rebecca Nash

Ph 626.969.8449 Fax 626.969.7299

E-mail sales@elnativogrowers.com

URL http://www.elnativogrowers.com

Private, wholesale, retail, Internet, $\mathrm{M}-\mathrm{F}$

7:30-4 Sat 7-11, since 1996. Plants: $60 \%$

native, $60 \%$ propagated; container; grass.
El Nativo Growers produces hundreds of species native to California, including many grass and grass-like species. Our selection is diverse enough to fit into any garden, landscape, revegetation, or erosion control project. Our expert staff is ready to meet your restoration and revegetation needs. Contract Growing.

ELFS Landscaping Inc

296 Walnut St, Rochester NH 03867

Contact Lenny Foss

Ph 603.332.8324 Fax 603.332.1523

E-mail elfs@metrocast.net

Private, retail. Plants.

Elk Mountain Nursery

PO Box 599, 142 Ward Cove Rd,

Ashville NC 28804

Contact Craig Mailloux

Ph 828.683.9330

E-mail craig@elkmountain.com

Private, wholesale. Plants.

Elk Ridge NatureWorks LLC

283 Elk Ridge Ln, Grantsville MD

21536

Contact Liz McDowell

Ph 301.895.3686 Fax 301.895.3686

E-mail info@elkridgenatureworks.com

Private, retail, mail. Plants.

Elkhorn Native Plant Nursery

1957 B Hwy 1,

Moss Landing CA 95039

Contact Rob de Bree

Ph 831.763.1207 Fax 831.763.1659

E-mail enpn@elkhornnursery.com

Private, wholesale, retail, contract. Plants.

Elmer Bailey Nursery

2617 Tonti Rd, Salem IL 62881

Ph 618.548.1603

Private, wholesale. Plants.

Elmore Roots Nursery

631 Symonds Mill Rd,

Wolcott VT 05680

Contact Zach or David

Ph 800.427.5268; 802.888.3305

Fax 802.888.8885

E-mail fruitpal@elmoreroots.com

Private, wholesale, retail. Plants.
Emerald Seed and Supply

67595 Hwy 74, lone OR 97843

Ph 800.826.8873

E-mailemeraldss@hughes.net

Private, wholesale, retail, mail. Seeds.

Enchanted Gardens

6420 FM 359, Richmond TX 77469

Ph 281.341.1206 Fax 281.341.5034

Private, wholesale, retail. Plants.

Enchanter's Gardens

HC 77 Box 108, Hinton WV 25951

Contact Peter Heus

Ph 304.466.3154

E-mail enchantersgarden@gmail.com

Private, wholesale, retail, by appt, since

1995. Plants, Seeds. No mail orders.

Consultation, seed-collecting.

Enders Greenhouse

104 Enders Dr, Cherry Valley IL 61016

Contact Shannon

Ph 815.332.5255 Fax 815.332.5255

E-mail info@endersgreenhouse.com

Private, wholesale, retail, mail. Plants.

Engel's Nursery Inc

2080 64th St, Fennville MI 49408

Contact Jim Engel

Ph 269.543.4123 Fax 269.543.4123

E-mail info@engelsnursery.com

Private, wholesale, contract. Plants, Seeds.

ENVIRONMENTAL CONCERN INC

PO Box P, 201 Boundary Ln, St

Michaels MD 21663

Contact Leslie Hunter-Cario

Ph 410.745.9620 Fax 410.745.4066

E-mail nursery-sales@wetland.org

URL http://www.wetland.org/

Private, wholesale, retail, $M-F-4: 30$ and by

appt, since 1972 . Plants: $100 \%$ native,

$100 \%$ propagated; container; tree, shrub, herbaceous, grass.

Environmental Concern promotes the public understanding and stewardship of wetlands with the goal of improving water quality and enhancing nature's habitat through wetland outreach and education, native species horticulture, and the restoration, construction, and enhancement of wetlands.

See ad on page 169. 
Environmental Equities Inc

PO Box 7180, Hudson FL 34674-7180

Contact Nancy Desmond

Ph 727.992.8905 Fax 813.856.1519

E-mail enveq_nd@hotmail.com

Private, wholesale, retail. Plants.

Environmental Seed Producers

PO Box 2709, Lompoc CA 93438

Contact Angela Lavie

Fax 805.735.8798

E-mail esp@espseeds.com

Private, wholesale. Seeds.

Envirotech Nursery

5380 Township $143 \mathrm{NE}$,

Somerset OH 43783

Contact Carla Stimmel

Ph 740.743.1669 Fax 740.743.1644

E-mail nursery@envirotechcon.com or

info@envirotechcon.com

Private, wholesale, retail, M-F 9-5, since 1994.

Plants: $100 \%$ native, $100 \%$ propagated.

\section{ERNST CONSERVATION SEEDS}

9006 Mercer Pike, Meadville PA 16335

Contact Sales/Customer Service

Ph 800.873.3321 Fax 814.336.5191

E-mail sales@ernstseed.com

Private, wholesale, retail. Seeds.

Ernst Seeds now supplies native Southeastern ecotypes and mixes as well as Northeastern ecotypes and mixes. Call, fax, or e-mail our knowledgeable sales staff now.

See ad on page 169.

Evergreen Nursery

PO Box 38, 26479 Hwy 74,

Kittredge CO 80457

Contact Tammy

Ph 303.674.2132 Fax 303.674.0608

E-mail info@evergreen.nursery.com

Private, retail. Plants.

Evergreen Nursery Company Inc

5027 County $T$ T,

Sturgeon Bay WI 54235

Contact Vickey Vanderhoof

Ph 920.743.4464 Fax 920.743.9184

E-mail vickey@evergreennurseryco.com Private, wholesale. Plants.

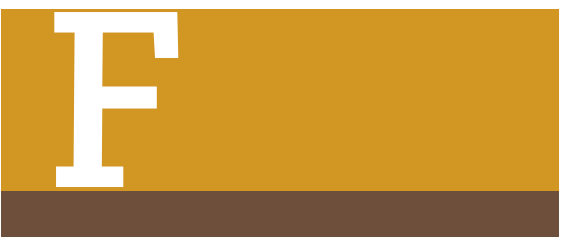

Fairview Evergreen Nurseries Inc PO Box E, 7463 W Ridge Rd, Fairview PA 16415

Ph 814.474.5712 Fax 800.343.6819

Private, wholesale. Plants.

Fairweather Gardens

PO Box 330, Greenwich NJ 08323

Contact Robert Hoffman or

Robert Popham

Ph 856.451.6261 Fax 856.451.0303

Private, retail, mail. Plants.

Fancy Fronds

PO Box 1090, 40830 172nd St SE,

Goldbar WA 98251

Contact Judith I Jones

Ph 360.793.1472 Fax 360.793.4243

E-mail judith@fancyfronds.com

Private, wholesale, retail. Plants.

Far North Tree and Seed

Company

PO Box 531, 5150 N Gina Dr,

Palmer AK 99645

Contact Nick Mihalow

Ph 907.745.4024 Fax 907.745.4024

E-mail nick@farnorthtreeandseed.com

Private, wholesale. Seeds.

Far Pastures Nursery

26929 115th Ave NE,

Arlington WA 98223-5645

Contact Carla Helms

Ph 360.435.4300 Fax 360.435.0819

E-mail farpastures@aol.com

Private, wholesale, retail, contract. Plants.

Far West Bulb Farm

14499 Lower Colfax Rd,

Grass Valley CA 95945

Contact Nancy or Ames Gilbert

Ph 530.272.4775

E-mail nancyames@spiralemail.com

Private, mail. Plants.
Faucette Tree Farm Inc

513 Audubon Dr,

Spartanburg SC 29302

Contact Bob Faucette or Jack Mills

Ph 864.585.2244 Fax 864.585.2244

E-mail crfaucette@gmail.com

Private, wholesale. Plants.

FEDER PRAIRIE SEED COMPANY

1740 Industrial $\mathrm{Dr}$,

Blue Earth MN 56013-9608

Contact Bill Olson

Ph 507.526.3049 Fax 507.526.3509

E-mail feder@bevcomm.net

URL http://www.federprairieseed.com

Private, wholesale, retail, mail, contract, M-F

$8-5$, since 1987 . Seeds: $100 \%$ native, $95 \%$

propagated, $5 \%$ wild collected; herbaceous, grass.

Producers of native grass and forb seeds and plants. All Southern Minnesota ecotypes.

Fernwood Nursery

6855 Tunnel Loop Rd,

Grants Pass OR 97526

Ph 541.472.0669 Fax 541.472.0689

E-mail fernwood@vsisp.net

Private, wholesale, retail. Plants.

Ferris Nursery

PO Box 258, 415 SE 98th St,

Newport OR 97365

Contact Rennie Ferris

Ph 541.265.5709 Fax 541.365.5105

E-mailferris@casco.net

Private, wholesale, retail. Plants, Seeds.

Fiddlehead Creek

7381 State Rt 40, Hartford NY 12838

Contact Emily DeBolt

Ph 518.632.5505

E-mail emily@fiddleheadcreek.com

Private, retail, Sat-Sun 10-5. Plants: 100\% native; container; shrub, herbaceous, grass, wetland/aquatic. Seeds: $100 \%$ native; shrub, herbaceous, grass.

Fieldstone Gardens Inc

55 Quaker Ln, Vassalboro ME 04989

Contact Steve Jones

Ph 207.923.3836

E-mail steve@fieldstonegardens.com

Private, retail. Plants. 


\section{Specializing in native wetland species since 1972.}

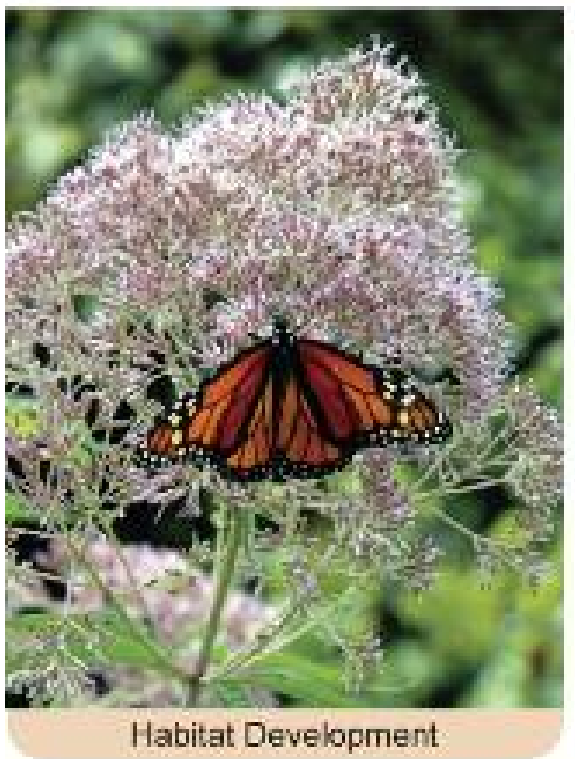

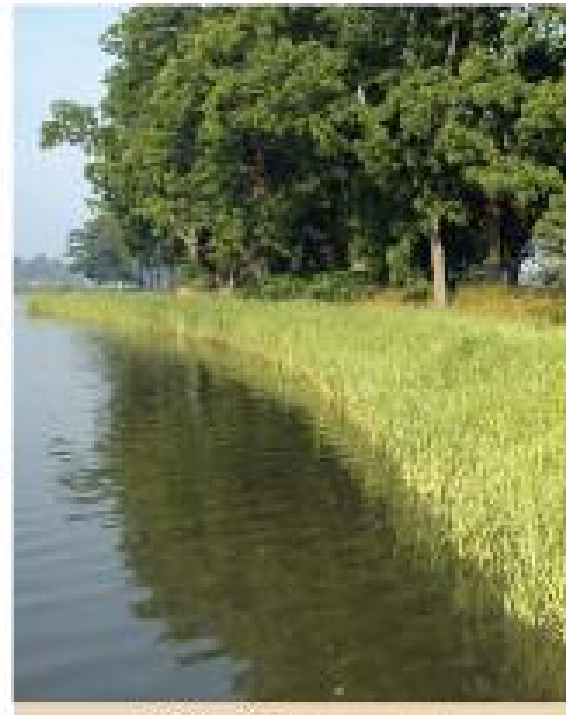

Buffer Management

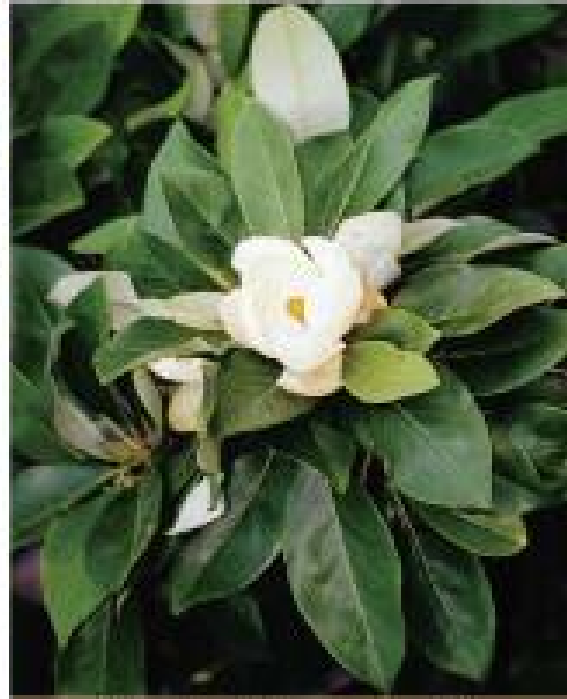

Shoreline Erosion Control

\section{Environmental Concern \\ P. O. Box P - 201 Boundary Lane \\ St. Michaels, MD 21663

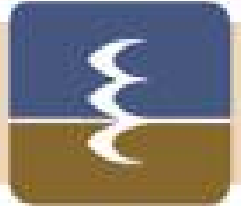 \\ Phone: $410-745-9260$ \\ Fax: $410-745-4066$ \\ Website: www wetkand org}

Finders Keepers Plants

5031 Whippoorwill Rd, Sebring FL 33872-6384

Contact Barbara Kircher

Ph 863.382.6553 Fax 863.382.9530

E-mail finderskeepersplants@hotmail.com

Private, wholesale. Plants.

Fir Run Nursery

15102 91st Ave Court E, Puyallup WA 98375

Contact Mike Fenmore

Ph 253.848.4731 Fax 253.445.4988 E-mail firrun@msn.com

Private, wholesale. Plants.

Firetrail Nursery

3107 140th St NW, Marysville WA 98271

Contact Linda Date

Ph 360.652.9021 Fax 360.652.8932

E-mail firetrailnursery@hughes.net

Private, wholesale, 9-4, by appt, since 1985. Plants: 95\% native, 100\% propagated.

Firstline Seeds Inc

11703 Rd 1 SE, Moses Lake WA 98837

Contact Kirk Jungers

Ph 509.765.1772 Fax 509.766.1861

E-mail firstlineseeds@yahoo.com

Private, wholesale, retail. Seeds.

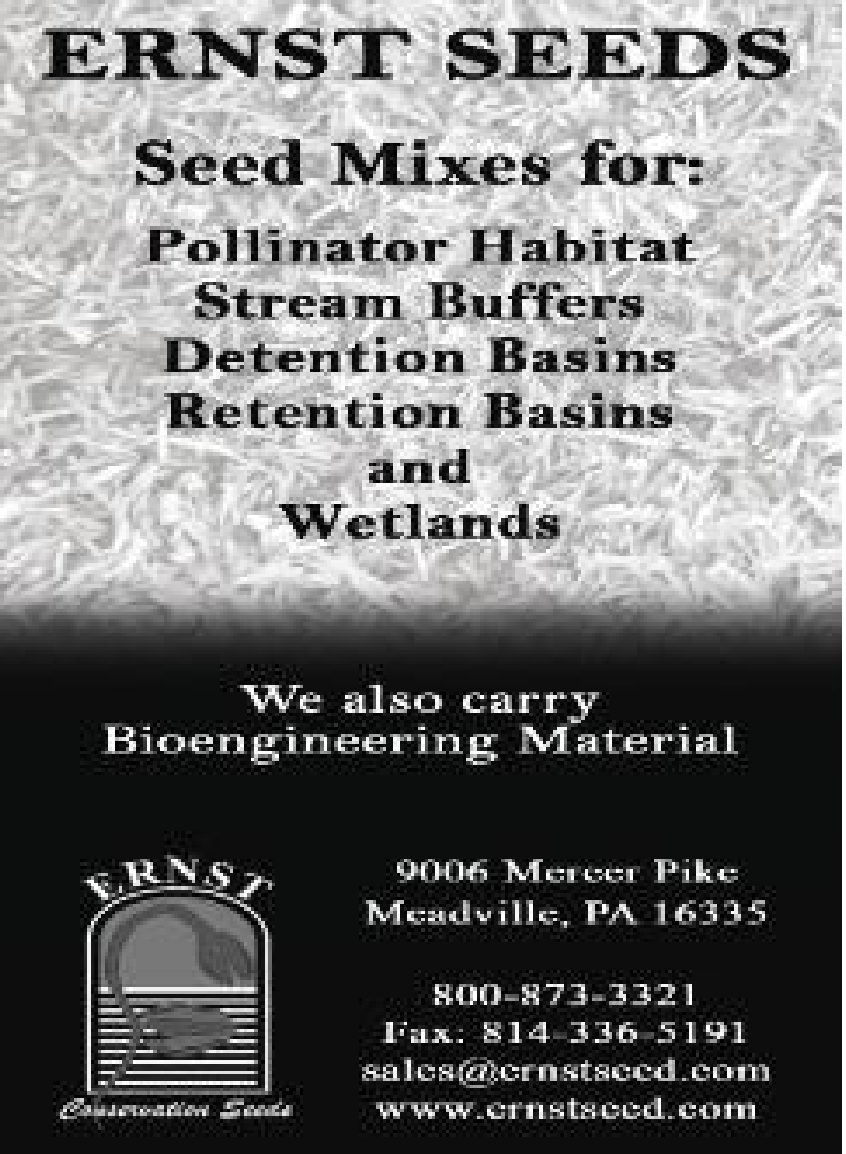


Flagstaff Native Plant and Seed 400 E Butler, Flagstaff AZ 86001 Contact Nigel Sparks Ph 928.773.9406 Fax 928.773.0107 E-mail info@nativeplantandseed.com Private, wholesale, retail, contract. Plants, Seeds.

Flagstaff Native Plant Nursery $1107 \mathrm{~N}$ Navajo Dr, Flagstaff AZ 86001 Ph 520.774.3584

Private, wholesale, contract. Plants.

Flickingers Nursery

Box 245, Rt 85, Sagamore PA 16250

Contact Tom Flickinger

Ph 800.368.7381 Fax 724.783.6528

E-mail flicknursery@gmail.com

Private, wholesale, retail, mail. Plants.

Flint River Nursery

9850 River Rd, Byromville GA 31007

Contact Jeff Fields

Ph 229.268.7308 Fax 229.268.1819

E-mail fields@gfc.state.ga.us

State, wholesale. Seeds.

Flora Lan Nursery

7940 NW Kansas City Rd,

Forest Grove OR 97116

Contact Larry Landauer

Ph 503.348.8464

E-mail landauer@coho.net

Private, wholesale. Plants.

Floral Native Nursery Inc

2511 Floral Ave, Chico CA 95973

Contact Germain Boivin or

Zeb Puterbaugh

Ph 530.892.2511 Fax 530.892.2511

E-mail canative@shocking.com

Private, wholesale, retail, contract, T-Sun

8:30-5:30, since 1998. Seeds: grass, herba-

ceous, wetland.

Florida Aquatic Nurseries Inc

700 S Flamingo Rd,

Ft Lauderdale FL 33325

Contact Marsela

Ph 954.472.5120 Fax 954.472.5446

E-mail admin@floridaaquatic.com

Private, wholesale, contract. Plants.
Florida Division of Forestry-

Andrews Nursery

PO Drawer 849, Chiefland FL 32644

9850 NW 42 Ct, Chiefland FL 32626

Contact Steven Gilly

Ph 352.493.6096 Fax 352.493.6084

E-mail gillys@doacs.state.fl.us

State, wholesale, retail, mail, M-F 8-5, since 1956. Plants.

Florida Keys Native Nursery

102 Mohawk St,

Tavernier FL 33070

Contact Kim

Ph 305.852.2636 Fax 305.852.2613

Private, wholesale, retail, mail. Plants.

Florida Native Flora Inc

PO Box 2291, Lakeland FL 33806

Contact Cindy Hill

Ph 863.853.8695 Fax 863.853.8695

E-mail FLNativeFlora@aol.com

Private, wholesale. Plants.

Florida Native Plants Inc

730 Myakka Rd, Sarasota FL 34240

Contact Laurel Schiller

Ph 941.322.1915 Fax 941.322.0208

E-mailfmplants@mailmt.com

Private, retail. Plants.

Foliage Gardens

2003 128th Ave SE, Bellevue WA 98005

Contact Sue Olson

Ph 425.747.2998 Fax 425.643.6886

E-mail foliageg@juno.com

Private, retail, mail. Plants.

Ford Seed Company Inc

2918 Woody Dr, Boise ID 83703

Contact Ken Ford

Ph 208.342.8088

E-mail9kss9@cable1.net

Private, wholesale, retail. Seeds.

Forest Floor Recovery Nursery

PO Box 89, Lummi Island WA 98262

Ph 360.758.2778 Fax 360.758.2778

E-mail ffrn1234@aol.com

Private, wholesale, mail. Plants.
Forest Seeds of California

1100 Indian Hill Rd,

Placerville CA 95667

Contact Bob

Ph 530.621.1551 Fax 530.621.1040

E-mail forestseeds@directcon.net

Private, wholesale, retail. Seeds.

Forestfarm

990 Tetherow Rd,

Williams OR 97544-9599

Contact Ray Prag

Ph 541.846.7269 Fax 541.846.6963

E-mail plants@forestfarm.com

Private, retail, mail, contract. Plants.

Foreverflora Palm Nursery

11004 SW 67th St,

Gainesville FL 32608

Ph 352.376.7762

E-mail cmeister@gnv.ifas.ufl.edu

Wholesale, contract. Plants.

FORREST KEELING NURSERY INC

PO Box 135, 88 Forrest Keeling Ln,

Hwy 79 S, Elsberry MO 63343

Contact Wayne Lovelace

Ph 800.356.2401 Fax 573.898.5803

E-mail info@fknursery.com or

wlovelace@fknursery.com

URL http://www.fknursery.com

Private, wholesale, retail. Plants.

Innovators of R.P.M. (Root Production Method), a multistep, natural root pruning process, which promotes lateral root development. Results are successful transplanting, accelerated growth, and early fruitland root production. More than 250 varieties. Since 1949.

\section{See ad on page 171.}

Fort Apache Agency-White

Mountain Apache

Box 560, White River AZ 85941

Contact Maurice Williams

Ph 928.338.5312; 928.338 .4346

Fax 928.338 .5385

Tribal, wholesale, retail. Plants.

Fort Pond Native Plants

PO Box 5061, 26 S Embassy St, Montauk NY 11954

Contact Jim Grimes

Ph 631.668.6452 Fax 631.668.6439

E-mail info@nativeplants.net

Private, retail, 8-5, since 1993. Plants. 


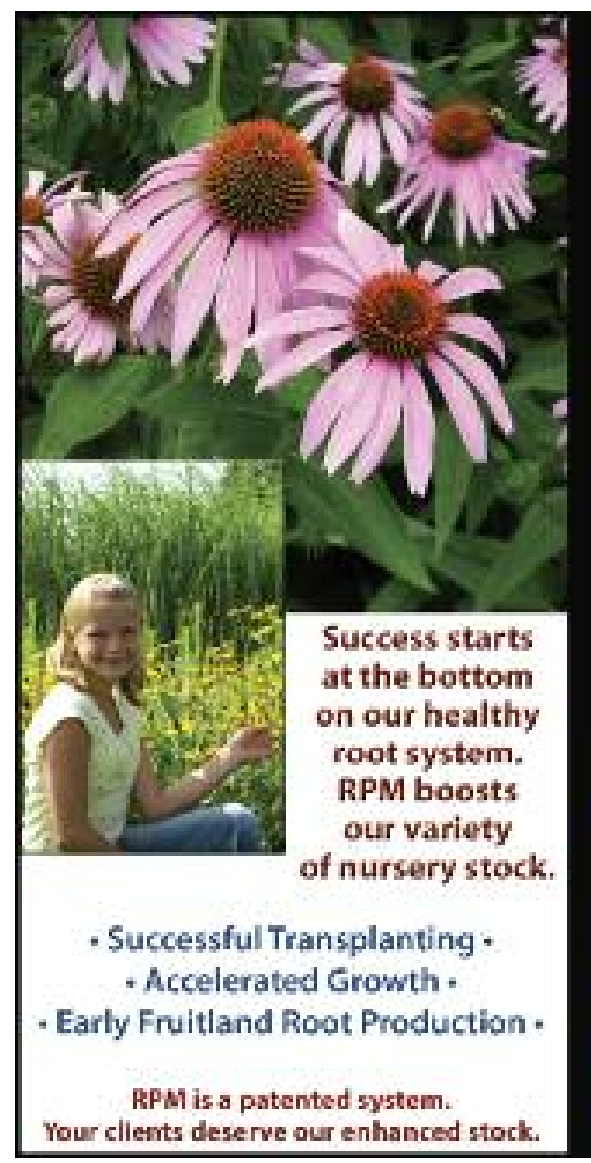

Fossil Creek Nursery

7029 S College Ave, Fort Collins CO 80525

Contact Brenda

Ph 970.226.4924 Fax 970.223.6348

E-mail info@fossilcreeknursery.com

Private, wholesale, retail. Plants.

Foster Rambie Grass Seed

PO Box 5386, 804 Airline Dr \#1,

Uvalde TX 78802-5836

Contact Johnny Rambie

Ph 830.278.2711

Private, retail. Seeds.

Found Well Farm

885 Eastlake Blvd, Carson City NV

89704

Ph 775.849.0213 Fax 775.849.2058

Private, retail, contract. Plants.

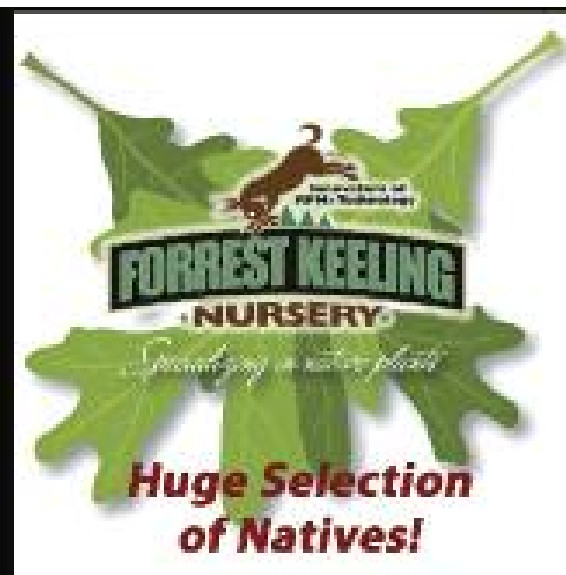

\section{All of our stock \\ is seed source-tracked. Check out our list!}
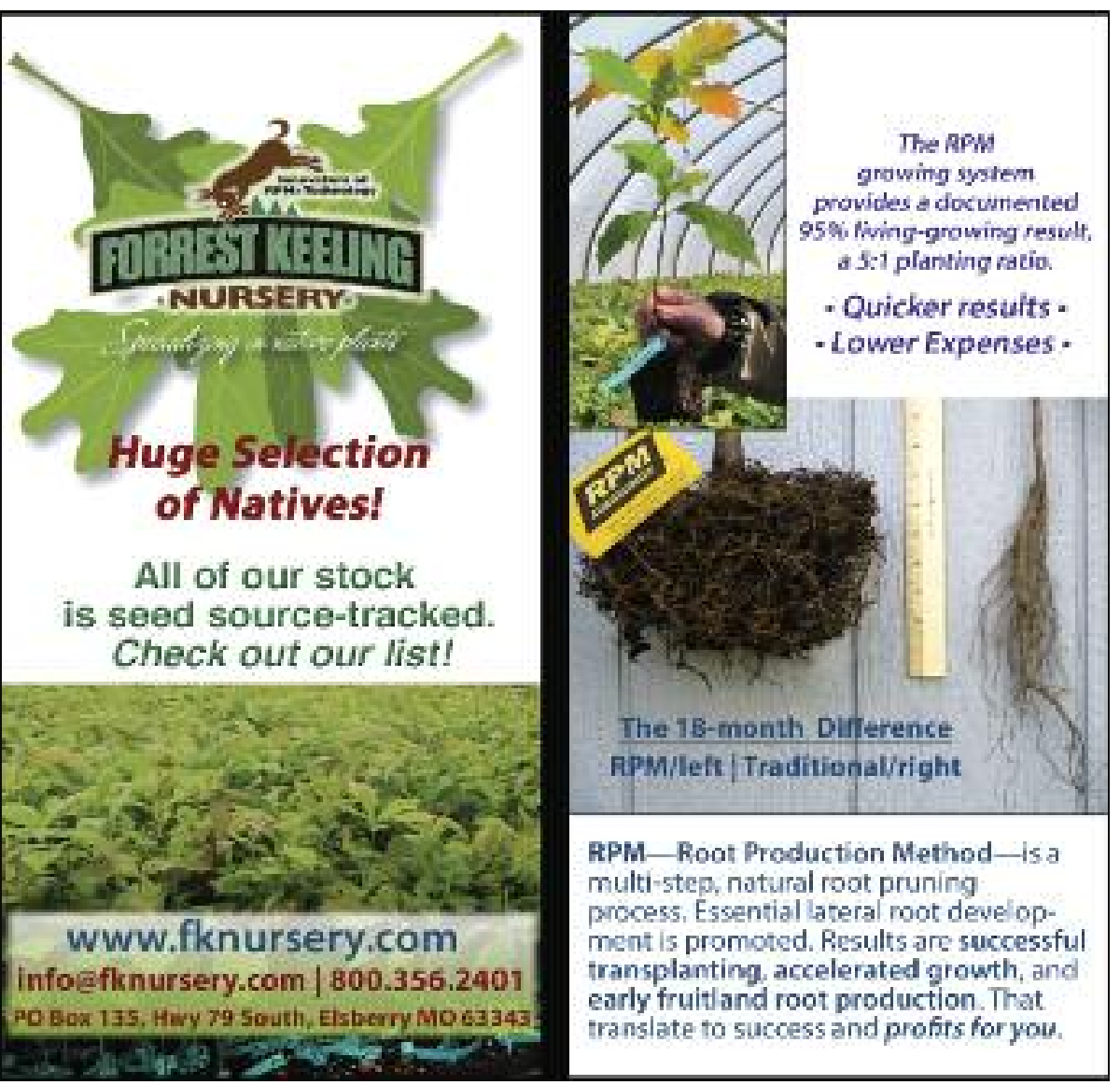

FOURTH CORNER NURSERIES

5652 Sand Rd, Bellingham WA 98226

Contact Julie Whitacre or Todd Jones

Ph 360.592.2250; 800.416.8640

Fax 888.506.1236

E-mail sales@fourthcornernurseries.com URL http://www.fourthcornernurseries.

\section{com}

Private, wholesale, M-F 8-4, since 1982.

Plants, Seeds. Min: $\$ 100$.

We rely on indexed seed collections to propagate more than 450 different native species of trees, shrubs, bulbs, perennials, and emergents from regions across North America.

See ad on inside cover.

Fraser's Thimble Farms

175 Arbutus Rd,

Salt Spring Island BC V8K $1 \mathrm{~A} 3$

Contact Richard Fraser

Ph 250.537.5788 Fax 250.537.5788

E-mail thimfarm@telus.net

Private, retail, mail. Plants.
FRESHWATER FARMS

5851 Myrtle Ave,

Eureka CA 95503-9510

Contact Rick Storre

Ph 707.444.8261 Fax 707.442.2490

E-mail rstorre@freshwaterfarms.com URL http://www.freshwaterfarms.com Private, wholesale, retail, mail, contract, M-F 9-5 Sat 10-3, since 1985. Plants: wetland/aquatic. Seeds. Min: $\$ 150$.

In business more than 20 y. Emphasis on wetland and riparian plants for restoration. Contract growing available. Installation and site evaluations offered. Call for more information.

Freshwater Farms I

North Coast Native Seed Bank 5851 Myrtle Ave, Eureka CA 95503 Contact Cindy Pemberon

Ph 707.444.8261 Fax 707.442.2490

Private, wholesale, retail, mail, contract. Seeds. 
Friendly Natives Plants and Design

2105 Country View Ln NE,

Bainbridge Island WA 98110

Contact Lissa

Ph 206.387.5943

E-mail lissa@friendlynatives.net

Private, wholesale, retail. Plants.

Fritz Creek Gardens

PO Box 15226, 3858 Lake St,

Fritz Creek AK 99603

Contact Rita Jo Shoultz

Ph 907.235.4969 Fax 907.235.8116

E-mail ritajo@alaskahardy.com

Private, wholesale, retail. Plants.

Frosty Hollow Ecological

Restoration

PO Box 53, Langley WA 98260

Contact Marianne Edain

Ph 360.579.2332

E-mail wean@whidbey.net

Private, wholesale, retail, mail, contract, $\mathrm{M}-\mathrm{F}$ $9-5$, since 1982 . Seeds: $100 \%$ native, $100 \%$ wild collected. Min: $\$ 50$.

Consultants in ecological restoration.

Future Forests Nursery

PO Box 847, Kailua-Kona HI 96745

Contact jill Wagner

Ph 808.325.2377

E-mail trees@forestnursery.com

Private, wholesale, retail, M-F 8-4, since 1926.

Plants: $50 \%$ native, $100 \%$ propagated.

FW SCHUMACHER COMPANY INC PO Box 1023,

Sandwich MA 02563-1023

Contact Donald Allen

Ph 508.888.0659 Fax 508.833.0322

URL http://www.treeshrubseeds.com

Private, wholesale, retail, since 1926. Seeds.

Selling seeds of trees and shrubs since 1926. Ornamental, Christmas tree production, reforestation, natives, land reclamation. We custom-clean seed and are looking for new sources of seeds every season. For current seed prices and technical details, please visit our website.

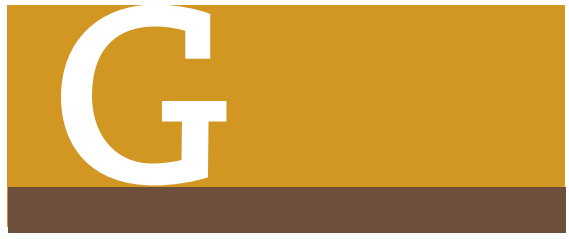

Gabriola Growing Company

171 Rosislos Blvd, Gabriola BC V0R 1X1

Contact Tom Collinson

Ph 250.247.8204

Private, wholesale, retail. Plants.

Garden Delights LLC I

Lazy K Nursery Inc

705 Wright Rd, 214 S Main St, Pine

Mountain GA 31822

Contact Ernest Koone III

Ph 706.663.7964 Fax 706.663.0939

E-mail info@lazyknursery.com

Private, wholesale, retail, mail. Plants.

The Garden Gate

3268 Fordham Parkway,

Gulf Breeze FL 32563

Contact Emily Peterson

Ph 850.932.9066 Fax 850.916.0200

E-mail thegardengategb@bellsouth.net

Private, retail. Plants.

\section{Garden of Delights}

14560 SW 14 St, Davie FL 33325-4127

Ph 954.370.9004 Fax 954.236.4588

Private, wholesale, retail, mail. Plants.

Garden Perennials

85261 Hwy 15, Wayne NE 68787

Contact Gail Korn

Ph 402.375.3615

E-mail gkorn@bloomnet.com

Private, retail. Plants.

\section{GARDENMAKERS}

PO Box 65, 240 Wethersfield St, Rowley MA 01969

\section{Contact Laura Militzer}

Ph 978.948.8481 Fax 978.948.8481

URL http://www.gardenmakers.com

Private, retail, mail, Internet. Seeds.

GardenMakers offers an unusual selection of perennial flower seeds including many woodland and native varieties as well as primulas, gentians, and ornamental grasses. Complete online catalog.
Gardens of the Blue Ridge

PO Box 10, 9056 Pittmans Gap Rd,

Pineola NC 28662

Contact Rob Fletcher

Ph 828.733.2417 Fax 828.733.8894

Private, retail. Plants.

Gary's Perennials LLC

1122 E Welsh Rd,

Maple Glen PA 19002-2224

Contact Andrea Steimberg

Ph 800.898.6653; 215.628.4070

Fax 215.628.0216

E-mail roots@garysperennials.com or aardvark@garysperennials.com

Private, wholesale, mail, Internet, contract, M-Sat 8-5, since 1984. Plants: 500,000/y, $35 \%$ native, $65 \%$ propagated; container, bareroot.

Field-grown perennials and cell paks with heavy root systems. Many rare and unusual varieties. High-quality plants, reliable service.

Gaspars Nursery

40620 158th St E, Lancaster CA 93535

Contact John or Evelyn Gaspars

Ph 661.264.4414

E-mail gasparsnursery@gtoast.net Private. Plants.

\section{GENESIS NURSERY INC}

23200 Hurd Rd, Tampico IL 61283

Ph 815.438.2220 Fax 815.438.2222

E-mail genesis_nursery@yahoo.com

Private, wholesale, mail, M-F 8-4:30, since 1992. Plants: $800,000 / y, 100 \%$ native, $100 \%$ propagated, $0 \%$ wild collected; herbaceous, grass, wetland/aquatic. Seeds: $25,000 \mathrm{lb} / \mathrm{y}$, $100 \%$ native, $100 \%$ propagated, $0 \%$ wild collected; herbaceous, grass. Min: varies.

Genesis Nursery Inc has more than 30 y experience in restoring native landscapes in the Midwest. Genesis is a family-owned corporation organized in Illinois in 1992 and is IDOT $D B E$ certified. We offer a variety of native seed mixes modeled on northern Illinois native communities and urban wetlands with approximately 300 seed species. We provide 250+ species of plant plugs for all landscapes and restorations, including wetland mitigation and stormwater management plantings. Custom and contract growing are available and custom seed mixes are welcome. Phone 877.817 .5325 or 815.438 .2220 and Fax 815.438.2222.

Seed mixes include our standard mixes, your custom mixes, IDOT and ISTHA mixes, or any mixes specific to projects you are bidding.

See ad on page 173. 
Georgia Pacific West Inc-

Forest Tree Nursery

90 W Redwood Ave, Fort Bragg CA 95437

Fax 707.964.3966

E-mail kdcovers@gapac.com

Forest Industry, wholesale. Plants, Seeds.

Gerard \& Greene

26225 S Woodlawn Ave, Crete IL 60417

Ph 708.672.1201

E-mailinfo@gerardandgreene.com

Private, wholesale, retail, mail. Plants.

GHW Weyerhaeuser Nursery

1123 Dinah's Landing Rd, Washington NC 27889

Contact Lucy Walker

Ph 252.946.7718 Fax 252.948.2759

E-mail lucy.walker@weyerhaeuser.com

Forest Industry. Plants.

Glacier Nursery Inc

4343 Montana Hwy 35, Kalispell MT 59901

Contact Brad Brown

Ph 406.755.2248; 800.700.8566

Fax 406.755.2249

E-mail gnursery@glaciernursery.com

Private, wholesale. Plants.

Glacier Oaks Nursery

8216 White Oaks Rd, Harvard IL 60033

Contact Mary T McClelland

Ph 815.482.7404 Fax 815.943.3511

E-mail glonursery@owc.net

Private, wholesale, contract. Plants.

Glass Mountain Forest Tree Nursery

PO Box 440, St Helena CA 94574

Ph 707.963.2372

Private, wholesale, retail. Plants.

Go Native Nursery

333 Cypress Ave, Moss Beach CA 94038

Ph 650.728.2286 Fax 650.728.3067

Private, wholesale, retail. Plants.

Golden State Growers

PO Box 14711, San Luis Obispo CA 93406

Ph 805.234.3751 Fax 805.596.0750

Private, wholesale, retail. Plants.

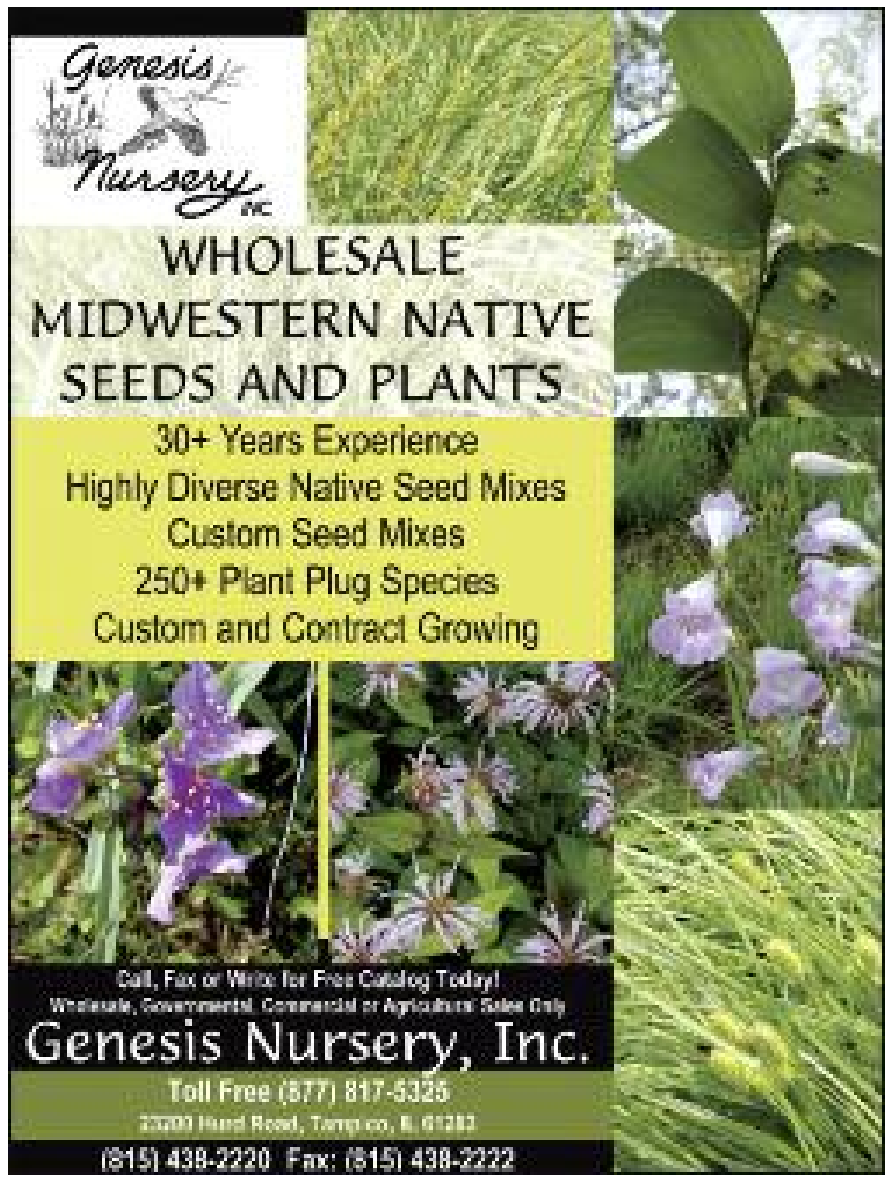

GONE NATIVE NURSERY

2704 SW Horseshoe Trail, Palm City FL 34990

Contact Joe Gilio

Ph 772.219 .7939

E-mail jlgilio@mac.net

Private, wholesale, retail, M-F 8-5, since 1982. Plants: $100 \%$ native,

$100 \%$ propagated, $1 \%$ wild collected; wetland/aquatic.

Gone Native Nursery provides top-quality native emergent and submergent aquatic vegetation for naturalizing manmade stormwater treatment systems and to improve water quality, increase animal diversity. Contract growing, restoration ecology.

Goodness Grows Inc

PO Box 311332, Elberton St (Hwy 77 N), Lexington GA 30648

Contact Richard Berry

Ph 706.743.5055 Fax 706.743.5112

E-mail admin@goodnessgrows.com

Private, wholesale, retail, mail. Plants.

Goodwin Creek Gardens

PO Box 83, 970 Cedar Flat Rd, Williams OR 97544

Contact Jim

Ph 541.846.7357 Fax 541.846.7357

E-mailinfo@goodwincreekgardens.com

Private, retail, mail, Internet, F-Sat Mar-Sep, since 1977. Plants: 5\%

native, $100 \%$ propagated; container. 
Gottlieb Gardens

8263 Huber Rd, Seguin TX 78155

Contact David Will

Ph 830.629.9876 Fax 830.629.9876

E-mail dewill@awsomenet.net

Private, wholesale. Plants.

Grand Isle Nursery

PO Box 350, 50 Ferry Rd,

South Hero VT 05486

Ph 802.372.8805; 800.400.grow

Fax 802.372.8305

E-mail info@grandislenursery.com

Private, wholesale, retail. Plants.

Grandiflora I San Felasco

Nurseries Inc

7315 NW 126 St, Gainesville FL 32653

Contact Alan Shapiro

Ph 352.332.1220; 800.933.9638

Fax 352.332.3113

E-mail

alan.shapiro@grandifloragrown.com

Private, wholesale. Plants.

GRANITE SEED

1697 W 2100 N, Lehi UT 84043

Ph 801.768.4422 Fax 801.768.3967

E-mail granite@graniteseed.com

URL http://www.graniteseed.com

Private, wholesale, M-F 8-5, since 1988.

Seeds: shrub, herbaceous, grass.

Granite Seed's inventory is made up of a diverse assortment of adapted local ecotypes including hundreds of hand-collected native

shrubs, grasses, and forbs.

Grassland West

908 Port Dr, Clarkston WA 99403

Contact Kevin Miller

Ph 509.758.9100 Fax 509.758.6601

E-mailkmiller@grasslandwest.com

Private, wholesale, retail, contract. Seeds.

Grasslander

RR 1, Box 56, Hennessey OK 73742

Contact Charles Grimes

Ph 405.853.2607 Fax 405.843.2607

E-mail grasslander@pldi.net

Private, wholesale, retail. Seeds.
Great Basin Natives

PO Box 114, 75 W 300,

Holden UT 84636-0114

Contact Merrill Johnson

Ph 435.795.2303 Fax 435.795.9111

E-mail merrille@crystalpeaks.com

Private, wholesale, retail, mail, Internet, contract. Plants.

GREAT BASIN SEED

323 E 750 S, Ephraim UT 84627

Ph 435.340.0442 Fax 435.283.6872

E-mail jason@cut.net

URL http://www.greatbasinseeds.com

Private, wholesale, retail, mail, Internet, contract, 8-5, since 2006. Plants: shrub, herbaceous, grass. Seeds: $70 \%$ native, $70 \%$ wild collected; shrub, herbaceous, grass.

Specializing in plant species for reclamation restoration and habitat improvement in the Great Basin and western US.

GREAT BEAR RESTORATION

310 N 4th St, Hamilton MT 59840

Contact Mark Rohweder or

Matthew Ogden

Ph $406.363 .5410 \times 116$

Fax 406.363.4349

E-mailmark@great-bear.biz

URL http://www.great-bear.biz

Private, wholesale, retail, mail, contract, $\mathrm{M}-\mathrm{F}$ 8-4:30, since 2006. Plants: 500,000/y, 100\% native, $100 \%$ propagated; container, bioengineering; tree, shrub, herbaceous, grass, wetland/aquatic. Seeds: $100 \%$ native, $100 \%$ propagated; tree, shrub, herbaceous, grass.

Great Bear Restoration provides full restoration services: seed collection, contract growing, installation, consulting, bioengineering. Great Bear Restoration (GBR) specializes in plant material from the Rocky Mountain, Intermountain West, Pacific Northwest, and Southwest Desert Regions. GBR provides full restoration services: custom seed collection, contract growing (some minor speculative production), installation, seeding, consulting, and bioengineering. We have 26 acres available for the production of large containers, wetland and upland sod. We provide plants from sourceidentified seed in container sizes ranging from plug trays to 15 gallon GrowBags, using a proprietary growth media enhanced with mycorrhizal inoculum. Our consulting services include research, planning, baseline vegetation surveys, and monitoring to provide optimum restoration practices for any site.
Great Lakes Nursery Company PO Box 693, Watervliet MI 49098 Ph 269.468.3323 Fax 269.468.6717 Private, wholesale. Plants.

Green by Nature

2115 Loff Rd, Castlegar BC V1N 4N9

Contact Annette Reed

Ph 250.304.5802

E-mail annette@greenbynature.ca

Private, wholesale, retail. Plants.

Green Desert Wholesale Nursery

79301 Ave 40, Indio CA 92203

Contact Chris Thompson

Ph 760.360.6937 Fax 760.772.1948

E-mail ctgdn@msn.com

Private, wholesale. Plants.

Green Hills Nursery

40805 Upper Nestuca Rd,

Beaver OR 97108

Contact Les

Ph 503.398.5965 Fax 503.398.5965

E-mail greenhills@oregoncoast.com

Private, wholesale, contract. Plants.

Green Images Native Landscape

Plants

1333 Taylor Creek Rd, Christmas FL

32709

Contact David

Ph 407.568.1333 Fax 407.568.2061

E-mail greenimage@aol.com

Private, wholesale, contract. Plants.

Green Isle Gardens

5243 Lido St, Orlando FL 32807

Contact Terry Godts

Ph 407.277.5159; 321.436.4932 Fax

352.429.3392

E-mailmarcg@netwide.net

Private, wholesale. Plants.

Green Man Gardens

6826 93rd Ave SE,

Mercer Island WA 98040

Contact Brett Johnson

Ph 206.232.5734 E-mail

bnbjohns@comcast.net

Private, contract. Plants. 
Green Oak Ranch Nursery

1237 Green Oak Rd, Vista CA 92083

Ph 760.727.0251 Fax 760.599.2665

Private, retail. Plants.

The Green Ranch

HC42, Box 1500, Boise ID 83706

Ph 208.342.8915; 208.392.4403 Fax

208.392.2256

Private, wholesale, retail. Plants.

Green Seasons Nursery

PO Box 539, 12340 SR 62,

Parrish FL 34219

Contact Roger Triplett

Ph 941.776.1605 Fax 941.776.0197

Private, wholesale. Plants.

Green Thumb Garden Shoppe LLC

913 Mira Ave, Crivitz WI 54114

Ph 715.854.3250

E-mail info@greenthumbgarden

shoppe.com

Private, retail. Plants.

The Greenery

14450 NE 16th PI, Bellevue WA 98007

Contact Lynn Watts Ph 425.641.1458

E-mail watts-greenery@msn.com

Private, retail. Plants.

Greenleaf Nursery-Oklahoma

28406 Hwy 82, Park Hill OK 74451

Contact Roger

Ph 800.331.2982

Private, wholesale. Plants.

Greenleaf Nursery-Texas

262 FM 2674 Rd, HCR 62 Box 73,

El Campo TX 77437

Contact Kevin Carroll

Ph 800.237.3147 Fax 979.543.1886

Private, wholesale. Plants: container.

Greenlee Nursery

6075 \#C Kimball Ave, Chino CA 91708

Contact John Greenlee

Ph 909.393.6544 Fax 909.393.6004

E-mail sales@greenleenursery.com

Private, wholesale. Plants.

Greenworld Inc

309 7th St NW, Sioux Center IA 51250

Contact Larry

Ph 712.722.2621 Fax 712.722.2649

Private, wholesale, retail. Plants.
Greer Gardens

1280 Goodpasture Island Rd,

Eugene OR 97401-1794

Contact Harold Greer

Ph 541.686.8266 Fax 541.686.0910

E-mail orders@greergardens.com

Private, retail. Plants.

Greg Peterson

6754 Partridge Dr NE,

Moses Lake WA 98837-9524

Contact Greg Peterson

Ph 509.765.7946 Fax 509.765.7946

E-mail wagrassman@hotmail.com

Private, wholesale, retail. Plants, Seeds.

Gress Evergreen Nursery

822 Acorn Trail, Oconto WI 54153

Contact Craig Bahrke

Ph 920.834.3044

Private, wholesale. Plants.

Grouse Springs Nursery

35649 Montana Hwy 35,

Polson MT 59860

Contact Caroline Clemans

Ph 406.887.2696

Private, retail. Plants.

GroWest Nurseries

1700 GroWest Ave,

Riverside CA 92504-5307

Contact Russ Kelly

Ph 951.780.1552 Fax 951.780.6676

E-mail rkelly@growest.com

Private, wholesale. Plants.

\section{GROWILD INC}

7190 Hill Hughes Rd,

Fairview TN 37062

Contact Terri Barnes

Ph 615.799.1910 Fax 615.799.1912

E-mail info@growildnursery.com

URL http://www.growildinc.com

Private, wholesale, retail, contract, M-F 8-4

Sat $8-12$ by appt, since 1997 . Plants:

$50,000 / y, 100 \%$ native, $100 \%$ propagated;

container, $B \& B$; tree, shrub, herbaceous.

Contract growing, consultation, landscape design and build, green roofs, rain gardens, and prairies. Growers of more than 900 species and cultivars of North American Natives. Visit us at www.growildinc.com.
GROWING SOLUTIONS

RESTORATION EDUCATION

INSTITUTE

PO Box 30081,

Santa Barbara CA 93130

4474 Calle Real

Santa Barbara CA 93111

Contact Karen Flagg

Ph 805.729.7003

E-mailinfo@growingsolutions.org

URL http://www.growingsolutions.org

Private, wholesale, retail, contract, by appt, since 1999. Plants: $75,000 / y, 100 \%$ native, $20 \%$ propagated, $80 \%$ wild collected; container, cuttings; tree, shrub, herbaceous, grass, wetland/aquatic. Seeds: 1000/y, 100\% native, $20 \%$ propagated, $80 \%$ wild collected; tree, shrub, herbaceous, grass. Min: $\$ 200$.

Our nonprofit organization is located in Santa Barbara County and dedicated to the restoration and preservation of California's remarkable botanic wealth and rich diversity. We provide educational programs, consultation services, and produce site-specific native plants and seeds for contract orders. We are a nonprofit organization, which seeks to educate people of all ages on the ethical stewardship of local watersheds and the restoration of degraded land. Our staff has 50 y combined experience specializing in restoration of wetlands, coastal sage scrub, grassland, riparian, maritime chaparral, seabird habitat, and oak woodland plant communities.

Guam Dept of Agriculture-

Forestry Division

192 Dairy Rd, Mangilao GU 96913

Ph 671.734.3948 Fax 671.734.0111

E-mail dlimti@mail.gov.gu

State. Plants.

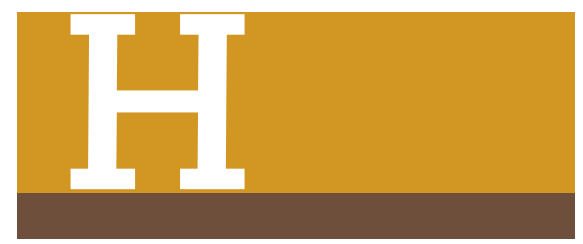

Habitat Restoration

PO Box 436, San Diego CA 92111

Ph 619.279.8769

Private, wholesale. Plants.

Habitat Solutions Inc

PO Box 14822, Pittsburgh PA 15228

Ph 412.853.7883 Fax 412.853.7883

Private, wholesale, mail. Plants. 


\section{HABITATS NATIVE PLANT}

NURSERY

PO Box 265, Silver Grove KY 41085

Contact Jacob Bartley, Nursery Manager

Ph 859.442.9414 Fax 859.442.9414

E-mail native@habitatsnursery.org

URL http://www.habitatsnursery.org

Private, wholesale, retail, mail, contract, M-F

9-5, since 1997. Plants: $100 \%$ native, $100 \%$

propagated; container, bareroot, cuttings, bio-

engineering; tree, shrub, herbaceous, grass,

wetland/aquatic. Seeds: $100 \%$ native, $100 \%$

propagated; tree, shrub, herbaceous, grass.

Min: $\$ 150$

Nonprofit. We specialize in local/genetic native plant materials and bulk seed of wetland, upland meadow, prairie, woodland, and select shrub species. Services include native landscape and restoration assessment, design, installation, and management for Indiana, Kentucky, and Ohio.

Halfmoon Growers Inc 1108 SW 186th St, 21704 SW 30th Ave, Newberry FL 32669

Contact Bruce McElroy

Ph 352.318.2853 Fax 352.472.6553

E-mail halfmoongrowers@yahoo.com Private, wholesale. Plants.

Hall Tree Services

51063 Hwy 290, Hempstead TX 77445

Ph 979.826.2747 Fax 979.826.9379

Private, wholesale. Plants.

Hamilton Native Outpost

16786 Brown Rd, Elk Creek MO 65464

Contact Rex or Amy Hamilton

Ph 417.967.2190 Fax 417.967.5934

Private, wholesale, retail. Plants, Seeds.

Hanchars Superior Trees

40 Hanchar Ln, Mahaffey PA 15757

Contact Frank

Ph 814.277.6674 Fax 814.277.6673

E-mail hanchar@pennswoods.net

Private, wholesale. Plants.

Hanging Dog Valley Nursery

2600 Boiling Springs Rd,

Murphy NC 28906

Contact John or Pam Strawn

Ph 828.837.7921 Fax 828.837.7921

E-mail strawn@directv.net

Private, wholesale, 8-5, since 1983. Plants.

Wholesale producer of $B \& B$ and container trees and shrubs with emphasis on native plants of the southern Appalachians.
Hard Scrabble Farms Inc

PO Box 281, Terra Ceia FL 34250

Contact Linda Osborne

Ph 941.722.0414 Fax 941.723.6258

E-mail wildflowers@pcsonline.com

Private, wholesale. Plants, Seeds.

Harold M Miller

Landscape Nursery

PO Box 989, 838 Ankeny Hill Rd SE,

Jefferson OR 97352

Contact Harold or Marty

Ph 503.399.1599 Fax 503.364.7552

E-mail hmillerlandscape@aol.com

Private, wholesale, contract. Plants.

Hartke Nursery

1030 N Warson Rd, Olivette MO 63132

Contact Don Walls or Cindy Collins

Ph 314.997.6679 Fax 314.997.6778

E-mail hartke@sbcglobal.net

Private, retail. Plants.

\section{HARTLAND NURSERY}

PO Box 439, 13737 Grand Island Rd,

Walnut Grove CA 95690

Contact Toni or Jeff Hart

Ph 916.775.4021 Fax 916.775.4022

Private, wholesale, retail. Plants.

E-mail hartlandnursery@mac.com

URL http://www.hartlandnursery.com

Private, wholesale, retail, contract, by

appt/Retail check website, since 1997.

Plants: $75 \%$ native, $100 \%$ propagated; con-

tainer, cuttings, bioengineering; tree, shrub,

herbaceous, grass.

Hartland Nursery specializes in growing California native and low-maintenance ornamentals, emphasizing wildlife habitat restoration, urban forests, and landscapes that have minimal maintenance requirements and drought-tolerant plantings. Services available include site-specific materials collection; designbuilt habitat restoration; contract (custom) growing; boat ecotours in northern California's scenic delta.

See ad on page 177.

Hartmann's Plant Company

PO Box 100, Lacota MI 49063

310 60th St, Grand Junction MI 49056

Contact Daniel P Hartmann

Ph 269.253.4281 Fax 269.253.4457

E-mail info@hartmannsplantcompany.com Private, wholesale, retail. Plants.
Hastings Smith River Nursery

PO Box 250, 800 Hwy 101 N,

Smith River CA 95567

Ph 707.487.3775 Fax 707.487.9301

E-mailstrees@gte.net

Plants.

Hawai'i Division of Forestry \&

Wildlife-Hilo Tree Nursery

PO Box 4849, Hilo HI 96720

Ph 808.933.4221

State. Plants.

Hawai'i Division of Forestry \& Wildlife_Kamuela State Tree

Nursery

66 12220A Lalmilo Rd,

Kamuela HI 96743

Contact Leslie Yokomizo

Ph 808.887.6061

E-mail ssuemann@yahoo.com

State, wholesale, retail, 8-4:30. Plants, Seeds.

Hawai'i Division of Forestry \& Wildlife-Kaua'i District Nursery 3060 Eiwa St Room 306, Lihue HI 96766-1875

State. Plants.

Hawai'i Division of Forestry \& Wildlife-Kokee State Park Native Plant Nursery

PO Box 100, Kekaha HI 96752

Contact Katie Cassel

Ph 808.274.3433 Fax 808.335.6131

E-mail rcp@aloha.net

State. Plants.

Hawai'i Division of Forestry \& Wildlife-Maui District Nursery

54 S High St Room \#101,

Wailuku Maui HI 96793

Contact Richard

Ph 808.873.3504 Fax 808.984.8111

State. Plants.

Hawai'i Division of Forestry \& Wildlife-Rare Plant Facility

1151 Punchbowl St Rm \#325,

Honolulu HI 96813

Contact Sheri S Mann

Ph 808.587.4172 Fax 808.587.0610

E-mail sheri.s.mann@hawaii.gov

State. Plants. 
Hawaii Palm Company

PO Box 459, Kapa'au HI 96755

Contact Becky Tarties

Ph 808.889.5906

E-mail hawaiipalmco@gmail.com

Private, wholesale. Plants.

Hawaiian Gardens

PO Box 1779, Kailua-Kona HI 96745

Contact Pati Barcas

Ph 808.329.5702 Fax 808.329.2082

E-mail hawaiiangardens@hialoha.net

Private, retail. Plants.

HE Nursery

1200 Old Rt 66, North Litchfield IL 61056

Ph 217.324.6191 Fax 217.324.5756

Private, wholesale, retail, contract. Plants.

Heartland Nursery Company

311 Main St, New Madrid MO 63869

Ph 573.748.5515 Fax 573.748.9155

E-mail hrtlnd_nsy_co@secglobal.net

Private, wholesale. Plants.

Heartland Restoration Services I Earth Source Inc 14921 Hand Rd, Fort Wayne IN 46818

Contact Eric Ummel

Ph 260.489.8511 Fax 260.489.8607

E-mail ericummel@earthsourceinc.net

Private, wholesale. Plants, Seeds.

Heep's Native Plant Nursery

1714 S Palm Court Dr, Harlingen TX 78552

Contact Mike Heep

Ph 956.457.6834

E-mail heep0311@yahoo.com

Private, wholesale, retail. Plants.

Helen's Native Plants \& Rustic

9121 2nd St NW, Albuquerque NM 87114

Contact Helen

Ph 505.804.4328

Private, wholesale, retail. Plants.

\section{Hartland Nursery}

Specializing in California

Native Plants and

Low-Maintenance Ornamentals

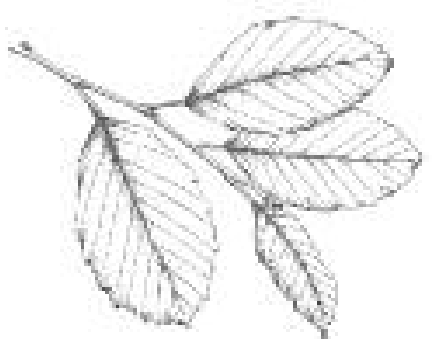

\section{Hart Restoration, Inc.}

Providing Practical Approaches to Complex Ecological Problems

\author{
PO Box 439 \\ Walnut Grove, CA 95690 \\ phoue 1916; 775-1021 \\ lax (916) $775-4022$ \\ weth. ww whastlandeurs. Ty.cous

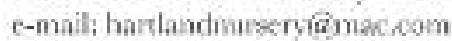

Hensler Nursery Inc

PO Box 58, 5715 N $750 \mathrm{E}$,

Hamlet IN 46532

Contact John Hensler

Ph 574.867.4192 Fax 574.867.4960

Private, wholesale. Plants.

HERITAGE SEEDLINGS INC

4194 71st Ave SE, Salem OR 97317

Contact Lynda Boyer

Ph 503.585.9835 Fax 877.643.0110

E-mail sales@heritageseedlings.com

URL http://www.heritageseedlings.com

Private, wholesale, contract, M-F 7-4:30 by appt, since 1986. Plants: $150,000 / y, 100 \%$ native, $100 \%$ propagated; container, bareroot, $B \& B$; tree, shrub, herbaceous, grass, wetland/aquatic. Seeds: 2000 $\mathrm{lb} / \mathrm{y}, 100 \%$ native, $100 \%$ propagated; herbaceous, grass. Min: $\$ 250$.

The native plant division of the company sells seed and plants of more than 85 species of source-identified Willamette Valley prairie grasses, forbs, and riparian plants. The seed and plugs are perfect for restoration and landscape use as well as western wildlife gardens. In addition, we feature numerous species and cultivars of North American trees and shrubs. Contract growing is also available.

See ad on page 179. 
Heronswood Nursery

300 Park Ave, Warminster PA 18974

Ph 877.674.4714 Fax 866.578.7948

E-mailinfo@heronswood.com

Private, retail, mail. Plants.

Heyne Custom Seed Service

16 E Main St, Walnut IA 51577-9745

Contact Roger

Ph 712.784.2430 Fax 712.784.2530

E-mail customseed@iowatelecom.net

Private, wholesale, retail. Seeds.

Hickory Hill Native Nursery Inc

27212 Hickory Hill Rd, Brooksville FL

34602

Contact Rick McDonnell

Ph 352.754.9701 Fax 352.799.7802

E-mail irckmhhnn@bellsouth.net

Private, wholesale, retail. Plants.

\section{HIGH COUNTRY GARDENS}

2904 Rufina St, Santa Fe NM 87507

Contact Ava Salman

Ph 800.925.9387 Fax 800.925.0097

E-mail plants@highcountrygardens.com URL

http://www.highcountrygardens.com

Private, wholesale, retail, 24/7, since 1984.

Plants: $70 \%$ native, $100 \%$ propagated;

shrub, grass.

Plants for the Western garden and beyond! Unique selection of waterwise plants including xeric perennials, grasses, shrubs, and coldhardy cacti and succulents.

High Country Nursery

HCR 77 Box 64, 835 Griswold Rd, Altamont TN 37301

Contact Everet Richards

Ph 931.692.3122 Fax 931.692.3210

Private, wholesale. Plants.

High Mountain Nursery

271 W Bitterbrush Ln,

Draper UT 84020

Contact Heidi Bullock

Ph 435.731.0107 Fax 435.615.6690

Private, wholesale, contract. Plants.

High Ranch Nursery

3800 Del Mar Ave, Loomis CA 95650

Ph 916.652.9261 Fax 916.660.0676

E-mail john@hrnursery.com

Private, wholesale, retail, contract. Plants.
HILD \& ASSOCIATES INC

326 Glover Rd, River Falls WI 54022

Contact George Hild

Ph 715.426.5131 Fax 715.426.9887

E-mail sales@hildandassociates.com or ghild@hildnatives.com

URL http://www.hildnatives.com

Private, wholesale, retail, contract, M-F 9-4 by appt, since 1986 . Plants: $100 \%$ native, $95 \%$ propagated, $5 \%$ wild collected; container, bareroot, bioengineering; herbaceous.

Hild \& Associates Inc is a native plant nursery specializing in wetland species. We provide standard nursery size potted, bareroot material and contract grow for native plantings, rain gardens, and shoreline stabilizations. We are a Blue Thumb partner! Our company also provides soft armor shoreline stabilization solutions through its EcoPatch erosion control blankets, EcoPatch aquatic planting patches, and prevegetated fiber logs. EcoPatch prevegetated erosion control blankets provide immediate vegetative cover, resulting in rapid development, effective soil and plant holding capacity, maximum seed germination and minimal loss prior to site placement. EcoPatch aquatic planting patches are excellent for planting emergent species. They are easy to plant and require no digging or substrate disturbance, while reducing loss of plants to waterfowl predation and wave action. High profile projects are immediately visually appealing, making EcoPatch an ideal choice for shoreline plantings.

Hill Country Natives

308 Mesa Oaks Dr, Leander TX 78641

Contact Emery

Ph 512.259.0253 Fax 512.259.0253

E-mail emitch@worldnet.att.net

Private, wholesale, retail. Plants.

Hillis Nursery Company Inc

92 Gardner Rd, McMinnville TN 37110 Contact Kelly

Ph 931.668.4364 Fax 931.668.7432

E-mail customerservice@hillisnursery.com Private, wholesale. Plants.

Hills Creek Nursery

826 Hills Creek Rd,

McMinnville TN 37110

Contact Jeff

Ph 931.668.8071 Fax 931.668.2062

E-mail hcreek@blomand.net

Private, wholesale. Plants.

See ad on page 179.
Hillside Nursery

107 Skinner Rd, Shelburne MA 01370

Contact Peter Joppe

Ph 413.625.9251 Fax 413.625.9251

E-mail info@hillsidenursery.biz

Private, retail. Plants.

Hines Hort Nursery

PO Box 1449, Vacaville CA 95696

Contact Joe Gray

Ph 707.446.4700 Fax 707.451.8861

E-mail jgray@hineshort.com

Private. Plants.

Ho'olawa Farms

PO Box 731, 3 Kahiapo PI,

Haiku HI 96708

Contact Anna Palomino

Ph 808.575.5099 Fax 808.575.5379

E-mail hoolawa@hawaii.rr.com

Private, wholesale, retail, contract, M-F 9-5

Sat-Sun by appt, since 1989 . Plants: $100 \%$ native.

Hobbs and Hopkins

1712 SE Ankeny, Portland OR 97214

Ph 800.345.3295 Fax 503.230.0391

E-mail info@protimelawnseed.com

Private, wholesale, retail. Seeds.

Holden Wholesale Growers

10374 Hazel Green Rd NE,

Silverton OR 97381

Contact Paul Holden

Ph 503.873.5940 Fax 503.873.8726

E-mail holdenwholesale@meritel.net

Private, wholesale. Plants.

Holland Wildflower Farm

PO Box 328, 290 O'Neal Ln,

Elkins AR 72727

Contact Julie Holland

Ph 479.643.2622 Fax 479.643.2622

E-mail hwildflowerfarm@cox.net

Private, wholesale, retail, mail, contract.

Seeds.

Holm Town Nursery Inc

PO Box 71565, 1301 30th Ave,

Fairbanks AK 99707

Contact Jim

Ph 907.451.8733 Fax 907.451.7248

E-mail holmtown@acsalaska.net

Private, retail. Plants. 
Homestead Gardens

743 W Central Ave,

Davidsonville MD 21035

Contact Tim Hamilton

Ph 800.300.5631; 410.956 .4777

Fax 410.956.2926

Private, wholesale, retail. Plants.

Hood Canal Nurseries

PO Box 36, Port Gamble WA 98364

Contact Mike Driscoll

Ph 360.297.7555 Fax 360.297.8446

E-mail nurseries@tscnet.com

Private, wholesale, contract. Plants.

HORIZON HERBS LLC

PO Box 69, Williams OR 97544

Contact Richo Cech

Ph 541.846.6704 Fax 541.846.6233

E-mail catalogrequest@budget.net

URL http://horizonherbs.com

Private, wholesale, retail, mail, M-F 9-5, since 1988. Plants:

$200,000 / y, 20 \%$ native, $100 \%$ propagated, $0 \%$ wild collected;

wetland/aquatic. Seeds: $20 \%$ native, $95 \%$ propagated, $5 \%$ wild

collected; tree, shrub, herbaceous, grass.

Extensive collection of medicinal herb seeds and plants from all over the world, common and rare, organically certified and nursery propagated. Visit our website at horizonherbs.com!

Horticultural Systems Inc

PO Box 70, Parrish FL 34219

Contact Chris Berry

Ph 800.771.4114; 941.776.2751 Fax 941.776.3700

E-mail dphelps@ecomgmt.com

Private, wholesale. Plants.

Hoyt Arboretum

4000 SW Fairview Blvd, Portland OR 97221

Contact Dan Moeller

Ph 503.823.4474 Fax 503.823.4213

E-mail dmoeller@ci.portland.or.us

Private, no public sales. Plants.

Hramor Nursery

2267 Murkey Rd, 829 W Fox Farm Rd, Manistee MI 49660

Contact Michael Morin

Ph 231.723.4846 Fax 231.723.5580

E-mail hramor@jackpine.com

Private, wholesale. Plants.

Hsu's Ginseng Enterprises Inc

PO Box 509, T 6819 Co Rd W, Wausau WI 54403

Contact Paul Hsu

Ph 715.675.2325 Fax 715.675.3175

E-mail info@hsuginseng.com

Private, wholesale. Plants, Seeds.
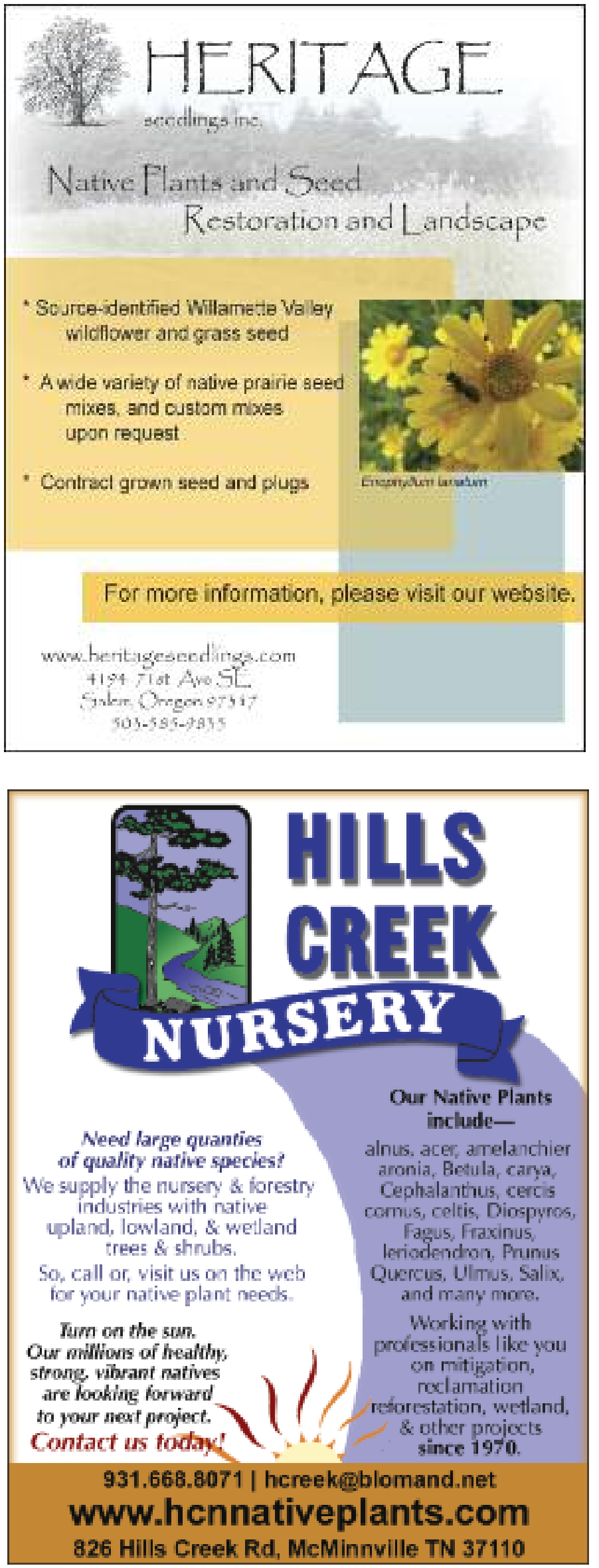
Huckleberry Lane Nursery

69117 Huckleberry Rd,

North Bend OR 97459

Contact Mel or Kathy Stewart

Ph 541.756.7328

Private, wholesale, by appt, since 1985.

Plants: $99 \%$ native, $100 \%$ propagated.

Contract growing, Oregon coast natives.

Hughes Water Gardens

25289 SW Stafford Rd,

Tualatin OR 97062

Contact Denise Carder

Ph 503.638.1004 Fax 503.638.9035

E-mail water@teleport.com

Private, wholesale, retail. Plants.

Humble Roots Farm and

Nursery LLC

Mosier OR 97040

Ph 503.449.3694

E-mail humbleroots@gorge.net

Private, wholesale, retail. Plants.

Husband Family Nursery

3712 Top Rock Ln,

Round Rock TX 78681

Ph 512.238.8694 Fax 512.238.8670

Private, wholesale, retail. Plants.

Hydra Aquatic

9 Gilcrease Ln, Los Lunas NM 87031

Ph 505.866.5027 Fax 505.866.SOIL

E-mail buyoaks@aol.com

Wholesale, mail, contract. Plants.

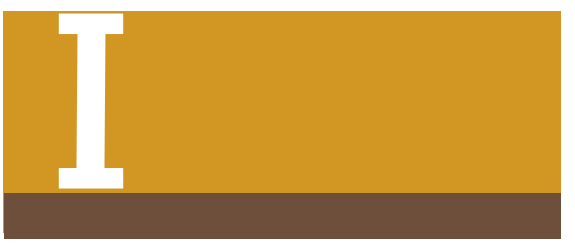

IAWISIL Forest Nursery

2433 N Cascade Rd, Cascade IA 52033

Contact Leo Freueh

Ph 563.852.5056 E-mail

leo@iawisil.com

Private, wholesale, retail. Plants.

Idaho Grimm Growers

PO Box 276, 395 S Broadway, Blackfoot ID 83221

Contact Alan DeGiulio

Ph 208.785.0830 Fax 208.785.0841

E-mailidgrimm@quest.net
IFA Nurseries Inc-

Canby Forest Nursery

1887 N Holly St, Canby OR 97013

Contact Dan Oconell

Ph 503.266.7825 Fax 503.266.7826

E-mail canby@ifanurseries.com

Private, contract. Plants.

IFA Nurseries Inc-

Corporate Office

136 NE Territorial Rd, Canby OR 97013

Contact Dave Stearns

Ph 503.855.3358 Fax 503.855.4539

E-mail corporate@ifanurseries.com

Private. Plants.

IFA Nurseries Inc-

Humboldt Nursery

4886 Cottage Grove Ave,

McKinleyville CA 95519

Contact Dan

Ph 707.839.3256 Fax 707.839.1975

E-mail humboldtnursery@humboldt1.com Private, wholesale. Plants.

IFA Nurseries Inc-

Klamath Falls Forest Nursery

1205 S Spring St,

Klamath Falls OR 97601

Contact Jacky Friedman

Ph 541.850.0952 Fax 541.850.0948

E-mail klamathfalls@ifanurseries.com

Private, contract. Plants.

Illinois Division of Forest

Resources-

Mason State Tree Nursery

17855 N Co Rd 2400 E,

Topeka IL 61567

Contact Dave Horvath

Ph 309.535.2185 Fax 309.535.3286

E-mail dave.horvath@illinois.gov

State, no public sales. Seeds.

Illinois Division of Forest

Resources-Union State Tree

Nursery

3240 State Forest Rd,

Jonesboro IL 62952

Contact Dan Bevil

Ph 618.833.6125 Fax 618.833.8123

State, retail. Plants.
Illinois Forest Products

8699 Arenzville Rd,

Beardstown IL 62618

Contact Kevin Massie

Ph 217.323.4540 Fax 217.323.9468

E-mail ifp@casscomm.com

Private, wholesale, retail. Plants.

Indian Trails Native Nursery

6315 Park Ln W,

Lake Worth FL 33467-6606

Contact Jane Thompson

Ph 561.641.9488 Fax 561.641.9309

E-mailinjntrails@aol.com

Private, wholesale, retail. Plants.

Indiana Division of Forestry-

Jasper-Pulaski State Nursery

15508 W 700 N, Medaryville IN 47957

Contact John Karstens

Ph 219.843.4827 Fax 219.843.6671

State, retail. Plants.

Indiana Division of Forestry-

Vallonia State Nursery

PO Box 218, 2782 W Co Rd 540 S,

Vallonia IN 47281

Contact Bob Hawkins

Ph 812.358.3621 Fax 812.358.9033

State, retail, M-F 8-4, since 1936. Plants.

Seedlings available for reforestation, windbreak establishment, wildlife enhancement, reclamation, and erosion control.

Inside Passage Seeds and Native Plants Services

PO Box 639, Port Townsend WA 98368

Contact Forest Shomer

Ph 360.385.6114 Fax 360.385.5760

E-mail inspass@whidbey.net

Private, wholesale, retail, mail. Seeds.

INTERLAKE FORAGE SEEDS LTD

Box 190, Fisher Branch MB ROC OZ0

Contact Paul Gregory

Ph 204.372.6920; 800.990.1390

Fax 204.372.6635

E-mail paul@interlakeforageseeds.com

URL http://www.interlakeforageseeds.com

Private, wholesale, 8-5, since 1979. Seeds:

$150,000 \mathrm{lb} / \mathrm{y}, 100 \%$ native; herbaceous, grass.

Our company focuses primarily on riparian and mesic northern phenotypes. We offer multipli-

cation contracts with the ability to custom clean all types of native seed. Climate-

controlled warehousing and an experienced

grower base help us maintain good customers. 
Intermountain Aquatics

PO Box 1115, 85 S Main, Driggs ID 83422

Contact Katherine Salsbury

Ph 208.354.3690 Fax 208.247.0941

Private, wholesale. Plants.

\section{Intermountain Cactus}

1478 N 750 E, Kaysville UT 84037

Contact Robert Johnson

Ph 801.546.2006

E-mail intermtcactus@xmission.com

Private, retail. Plants.

Intermountain Nursery

30443 N Auberry Rd, Prather CA 93651-9600

Contact Bonnie Bladen or Ray Laclergue

Ph 559.855.3113 Fax 559.855.8809

E-mail Bonnie@Intermountainnursery.com

Private, wholesale, retail. Plants.

Intermountain Seed Company

Box 62, 270 W 300 N, Ephraim UT 84627

Contact Eric Ph 435.283.4703 Fax 435.283.4388

E-mail ecc3@cisna.com

Private, wholesale. Plants, Seeds.

\section{INTERNATIONAL FOREST COMPANY}

1265 Georgia Hwy 133 N, Moultrie GA 31768-7165

Contact Wayne Bell

Ph 800.633.4506 Fax 229.890.0087

E-mailinfo@interforestry.com

URL http://www.interforestry.com

Private, wholesale, retail, $8-5$, since 1972 . Plants: $40,000,000 / y$, $100 \%$ native, $100 \%$ propagated, $25 \%$ wild collected; container; tree. Seeds: $5000 \mathrm{lb} / \mathrm{y}, 100 \%$ native, $100 \%$ propagated, $25 \%$ wild collected; tree. Min: $\$ 25$.

Native hardwood and conifer seedlings for the Southeast US. Wiregrass seedlings available. Conifer seeds available.

International Paper Company-Union Springs

SuperTree Nursery

686 Co Rd 28, Union Springs AL 36089

Contact Mark Parrot

Ph 334.474.3229 Fax 334.474.3246

E-mail Mark.Parrott@ipaper.com

Private, wholesale, retail. Plants.

Intervale Conservation Nursery

180 Intervale Rd, Burlington VT 05401

Contact Andrea Tursini

Ph 802.660.0440 x105 Fax 802.658.8075

E-mail andrea@intervale.org

Private, wholesale, retail. Plants.

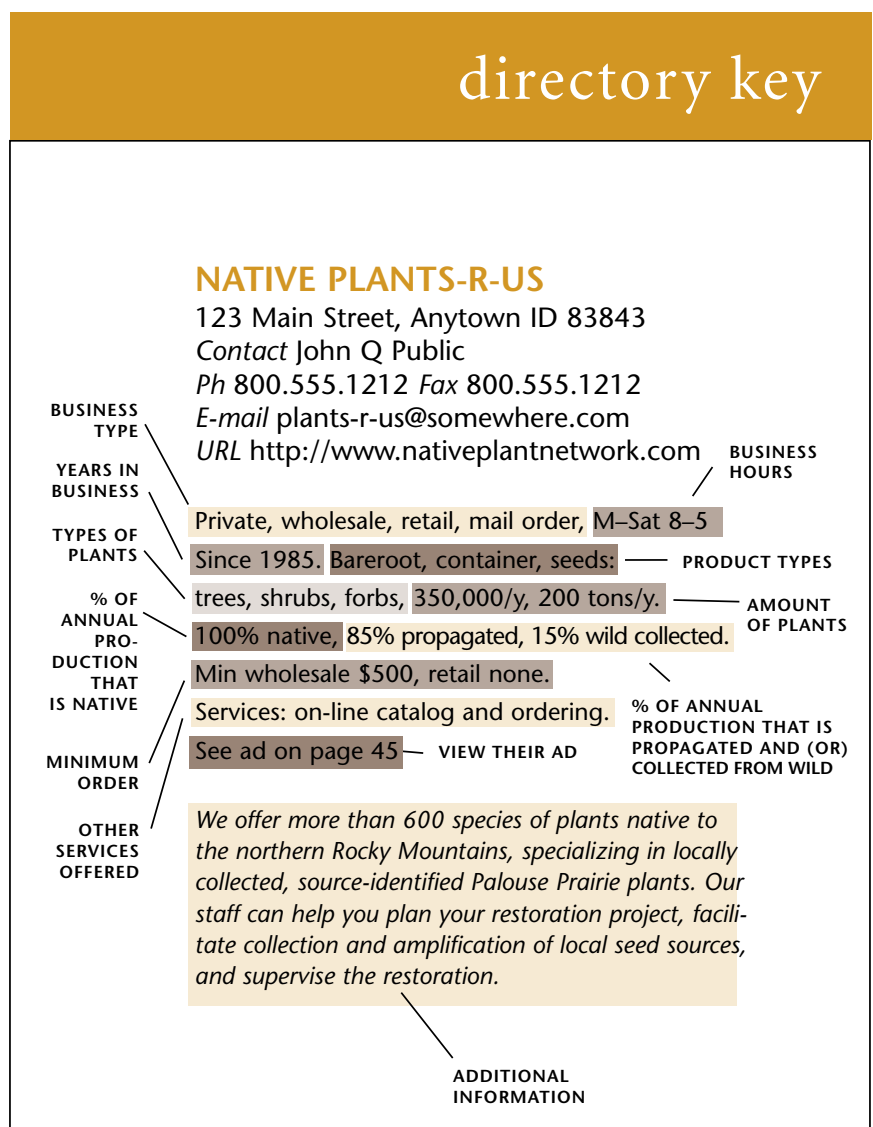

Io Makuahine

46281 Auna St, Kane'ohe HI 96744

Contact Tom Loudat

Ph 808.235.0578 Fax 808.235.5161

E-mail tomloud@earthlink.net

Private, wholesale, retail. Plants.

Ion Exchange Inc

1878 Old Mission Dr, Harper's Ferry IA 52146-7533

Contact Howard Bright

Ph 800.291.2143 Fax 563.535.7362

E-mail hbright@acegroup.cc or sales@ionxchange.com

Private, wholesale, retail, mail, Internet, contract, by appt, since 1988 . Plants: $100 \%$ native, $95 \%$ propagated, $5 \%$ wild collected; container, bioengineering. Seeds: $100 \%$ native, $95 \%$ propagated, $5 \%$ wild collected. Min: $\$ 10$.

Ion Exchange Inc is a native seed and plant nursery offering high quality native seed and live plant plugs, floating islands, ecolawn low-maintenance grass seed and consulting. We can help you "create your own beauty."

Iowa Dept of Natural Resources-

State Forest Nursery

2404 South Duff Ave, Ames IA 50010

Contact Roger Jacob

Ph 800.865.2477 Fax 515.233.1131

E-mail Roger.Jacob@dnr.iowa.gov

State, retail, mail, Internet. Plants. 
Itasca Greenhouse Inc PO Box 273, 26385 Blackwater Rd, Cohasset MN 55721

Contact William R Sayward Ph 800.538.8733 Fax 218.328.9843

E-mailinfo@itascagreenhouse.com Private, wholesale, retail. Seeds.

\section{ITASCA LADYSLIPPER FARM} SPANGLE CREEK LABS

21950 Co Rd 445, Bovey MN 55709

Contact Carol Steele

$\mathrm{Ph}$ 218.247.0245

E-mail ladyslippers@isp.com

URL http://www.spanglecreeklabs.com

Private, wholesale, retail, mail, Internet, since 1990. Plants: $10,000 / y, 85 \%$ native, $100 \%$ propagated; container, bareroot; herbaceous. Seeds.

We sell only Cypripediums propagated on-site in our lab to relieve the threat on wild populations. Cypripedium are perfect for wildflower gardens and for restoration projects. See our beautiful photos, species available, and order form on our website.

It's About Thyme Garden Center 11726 Manchaca Rd, Austin TX 78748 Ph 512.280.1192 Fax 512.280.6356 E-mail iathyme@yahoo.com Private, retail. Plants.

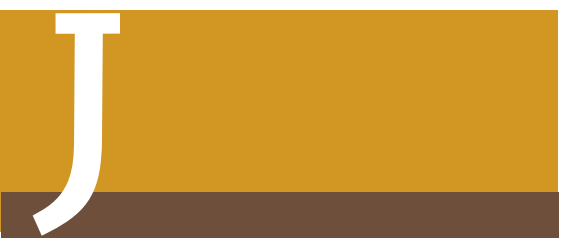

J\&J Transplant Aquatic Nursery PO Box 227, W 4980 Co Rd W, Wild Rose WI 54984

Contact Jim

Ph 715.256.0059 Fax 715.256.0039

E-mail jitransplant@yahoo.com

Private, wholesale, retail. Plants.

J Frank Schmidt \& Son Company PO Box 189, Boring OR 97009

Ph 503.663.4128 Fax 503.663.2121

E-mail talk-to-us@jfschmidt.com

Private, wholesale. Plants, Seeds.
James Reneau Seed Company

PO Box 40, 119 S Main,

Shamrock TX 79079

Contact James

Ph 806.256.3216 Fax 806.256.5335

E-mail reseedtex@yahoo.com

Private, wholesale, retail. Seeds.

Jane's Native Seeds

311 W Campbell St,

Frankfort KY 40601

Ph 502.750.1571

Private, wholesale, contract. Seeds.

Jansen's Specialty Nursery

20555 SE Webfoot Rd,

Dayton OR 97114

Contact Bob or Betty

Ph 503.868.7353 Fax 503.868.7353

Private, wholesale. Plants.

\section{JAYKER WHOLESALE}

NURSERY INC

4042 and 4740 W Chinden,

Meridian ID 83642

Contact Sherrill Jayker or Ed Hubbard

Ph 208.887.1790 Fax 208.887.9330

E-mailinfo@jayker.com

URL http://www.jayker.com

Private, wholesale, Sat 8-4:30 seasonally, since 1990 . Plants: $10 \%$ native, $100 \%$ propagated; tree, shrub, herbaceous, grass.

We are a wholesale grower of native xeric, and a full line of cold-hardy nursery stock, specializing in field-grown conifers and deciduous trees as well as container-grown trees, native plants, perennials, and ornamental shrubs. We are located in southern Idaho, near Boise, where the desert climate is harsh and perfect for growing tough, hardy nursery stock. Please visit our website for information and availability.

JD Irving Juniper Nursery 201 South West Rd, Juniper NB E7L 4S7 Contact Mike Boyd

Ph 506.246.5268 Fax 506.246.8110

E-mail dionne.maurice@jdirving.com Private, retail. Plants.

Jeff Anhorn Nursery

PO Box 2061, Livermore CA 94551

Contact Jeff Anhorn

Ph 800.303.0858; 925.447.0858

Private, wholesale, retail. Plants.
Jenkins \& Son Tree Farm 1014 Elmira Rd, Sandpoint ID 83864 Contact David Jenkins

Ph 208.265.5132 Fax 208.265.6148

E-mail jstreesdave@aol.com

Private, wholesale. Plants.

JF NEW NATIVE PLANT NURSERY

128 Sunset Dr, Walkerton IN 46574

Contact Jason Fritz

Ph 574.586.2412 Fax 574.586.2718

E-mail nurserysales@jfnew.com

URL http://www.jfnew.com

Private, wholesale, M-F 8-5. Plants: $100 \%$ native, $100 \%$ propagated; container, bareroot, cuttings, bioengineering; tree, shrub, herbaceous, grass. Seeds.

IF New is one of the leading native plant nurseries in the Great Lakes basin serving Illinois, Indiana, Kentucky, Michigan, Ohio, and Wisconsin. We grow prairie, wetland, and woodland plants and seeds. Additional services include design review, site inspections, installation, permitting, exotic weed control, and contract growing. Visit our website for additional information.

See ad on page 183.

JIFFY-PREFORMA

5401 Baumhart Rd, Ste B,

Lorain $\mathrm{OH} 44053$

Contact Don Willis or Todd Baker

Ph 800.323.1047 Fax 440.282.2853

E-mail d.willis@jiffygroup.com or t.baker@jiffygroup.com

URL http://www.jiffygroup.com Private, wholesale, M-F 8-6 Eastern, since 1993.

Services: Jiffy Forestry Pellets, Jiffy-7

Horticulture Pellets, Jiffy Peat Pots, Carefree Plugs, Preforma Plugs, Media SoilMix, Jiffy Tree Seed Promotional Kits.

See ad on page 185 .

JIM HARRINGTON SEEDS

1669 Ridge Rd, Lansing NY 14882

Contact Jim Harrington

Ph 607.533.7955

E-mail jim@jimharringtonseeds.com URL http://www.jimharringtonseeds.com Private, wholesale, $8-8$, since 1994 . Seeds: $45 \%$ native, $2 \%$ propagated, $40 \%$ wild collected; tree, shrub, herbaceous. Min: None; Charge for less than $1 / 4 \mathrm{lb}$.

For sale, all quantities of tree, shrub, and plant seeds for propagation. Global seed source contacts. Import/Export. Selling and buying seeds. Native plants of North America. 
Jim's Garden Center

2000 W Main, Sedalia MO 65301

Contact Jim Ph 660.826.4411 Fax 660.829.1311

E-mail jgcenter@iland.net

Private, retail. Plants.

JL Hudson, Seedsman

Box 337, La Honda CA 94020

Private, mail. Seeds.

JM Oak Tree Nursery

430 La Lata Pl, Buellton CA 93427

Contact Jerry Ph 805.688.5563

Private, wholesale, retail. Plants.

Joe Brown Native Seeds

7327 Hoefork Ln, Gloucester Point VA 23062

Contact Joe Brown Ph 804.642.0736

Private, wholesale, retail, mail. Seeds.

John Arnoldink Nursery

723 Old Orchard Rd, Holland MI 49423

Contact John Ph 616.335.9823 Fax 616.335.9823

E-mail johnarnoldink@hotmail.com

Private, wholesale. Plants.

John Deere Landscapes

5610 McGinnis Ferry Rd, Alpharetta GA 30005

Contact Randy Rankin Ph 770.442.8881 Fax 770.442.3214

E-mail rrankin@johndeerelandscapes.com

Private, retail. Plants.

Johnny's Selected Seeds

955 Benton Ave, Winslow ME 04901

Contact Rob Johnston

Ph 207.861.3900; 877.564.6697 Fax 207.738.6314

E-mail staff@johnnyseeds.com

Private, retail, mail. Plants, Seeds.

Johnson's Nursery

W 180 N6275 Marcy Rd, Menomonee Falls WI 53051

Ph 262.252.4988 Fax 262.252.4980

E-mail info@johnsonsnursery.com

Private, wholesale, retail. Plants.

Johnston Nurseries

Rd \#1 Box 100, 4842 Chambersville Rd,

Creekside PA 15732-9710

Contact Dave Johnston Ph 724.463.8456 Fax 724.465.5833

E-mail seedlings@hotmail.com

Private, wholesale, retail. Plants.

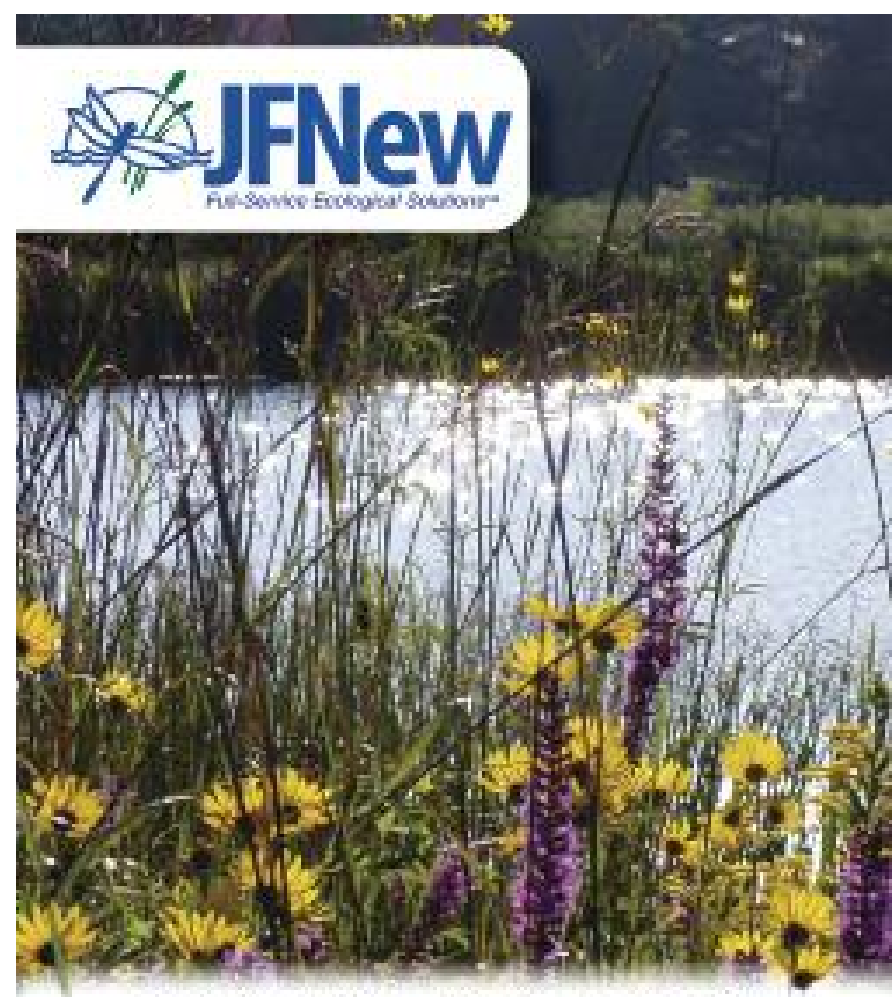

Native Seed Mixes - Rain Garden Materials Gallon Stock - Native Plant Plugs

nurserysales@JFNew.corm $\quad 574.586 .2412$ www.JFNew.com

Johnston Seed Company

PO Box 1392, 319 W Chestnut, Enid OK 73701

Contact Jean McVay

Ph 580.233.5800 Fax 580.249.5324

E-mail johnseed@johnstonseed.com

Private, wholesale, retail. Seeds.

Joshua Timberlands Nursery

29650 Comstock Rd, Elberta AL 36530

Contact Sam Campbell Ph 251.986.5210 Fax 334.986.4211

E-mail scampbell@molpus.com

Private. Plants.

Joshua's Native Plants \& Garden Antiques

502 W 18th St, Houston TX 77008

Contact Joshua Ph 713.862.7444 Fax 713.869.6911

E-mail Joshua@joshuasnativplants.com

Private, retail. Plants.

Joy Creek Nursery

20300 NW Watson Rd, Scappoose OR 97056

Ph 503.543.7474 Fax 503.543.6933

E-mail plants@joycreek.com

Private, retail, mail. Plants. 
Judd Creek Nursery

PO Box 13378, 20929111 th Ave SW,

Vashon WA 98070

Contact John Brown

Ph 206.463.9641 Fax 206.463.9641

E-mail jb4juddcreek@webtv.net

Private, wholesale, retail. Plants.

Julius Rosso Nursery Company PO Box 80345, 6404 Ellis Ave S,

Seattle WA 98108

Contact Gene Rosso

Ph 206.763.1888 Fax 206.762.2544

E-mail jerry0716@mindspring.com

Private, wholesale. Plants.

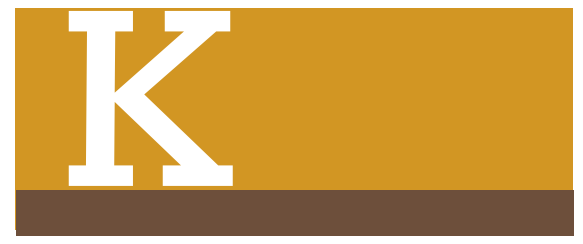

K\&C Silviculture Farms Ltd

PO Box 25019, Red Deer,

Joffre $A B$ T4R 2M2

Contact Tina Lambert

Ph 403.347.3002 Fax 403.347.3899

E-mail brad@silviculture.com

Private. Plants.

K\&C Silviculture Farms Ltd

PO Box 459, Oliver BC V0H 1T0

Ph 250.498.4974 Fax 250.498.2133

E-mail mail@silviculture.com

Private. Plants.

Kansas Forest Service

2610 Claflin Rd,

Manhattan KS 66502-1798

Contact Mark Haller

Ph 785.532.3300 Fax 785.532.3305

E-mail mhaller@ksu.edu

State, retail. Plants.

Kapoho Kai Nursery

RR 2 Box 4024, Pahoa HI 96778

Contact Jan Anderson

Ph 808.965.8839 Fax 808.965.9696

E-mail tidepool@verizon.net

Private, retail. Plants.
Karma's Forest Wholesale Nursery

and Organic Gardens

23223 Hwy 36, Cheshire OR 97419

Contact Lisa

Ph 541.998.2436 Fax 541.998.3437

E-mail karmasforest@yahoo.com

Private, wholesale, contract. Plants.

\section{KASTE SEED INC}

11779410 St SE, Fertile MN 56540

Contact Garth or Colleen Kaste

Ph 218.945.6738 Fax 218.945.6303

E-mail kaseinc@gvtel.com

Private, wholesale, retail, mail, M-F 8-5, since 1979. Plants, Seeds.

Kaste Seed Inc is a 2,300-acre native grass and wildflower seed production farm located in northwestern Minnesota. We offer high quality, northern-grown seed products to companies throughout the US. Contract production available; northern Great Plains ecotypes.

See ad on page 187.

Kauai Nursery and

Landscaping Inc

3-1550 Kaumualii Hwy, Lihue HI 96766

Contact Lelan Nishek

Ph 808.245.7747 Fax 808.245.9289

E-mail knl@kauainursery.com

Private, wholesale, retail. Plants, Seeds.

Kaw River Restoration Nurseries

701 E 22nd St, Lawrence KS 66046

Contact Elliot Duemler

Ph 785.842.3300 Fax 785.842.3313

E-mail krrn@appliedeco.com

Private, wholesale. Plants, Seeds.

Keefer Ecological Services Ltd

3816 Highland Rd,

Cranbrook BC V1C 6X7

Contact Michael Keefer

Ph 250.489.0917 Fax 250.489.4142

E-mail mike@keefereco.com

Private, wholesale. Plants.

Kelly Green Trees Inc

PO Box 10, 14399 N Wentz Rd

Marana AZ 85653

Contact Angel Murrieta

Ph 520.682.2616 Fax 520.682.3579

E-mail kellygreentrees@theriver.com

Private, wholesale. Plants.
Kenaitze Greenhouse

and Gardens

255 N Ames, Kenai AK 99611

Ph 907.283.3052 Fax 907.283.3052

Private, wholesale, retail. Plants.

Kendrick Forest Farm

1450 Round Prairie Creek,

Wilderville OR 97543

Ph 541.474.0217 Fax 541.474.0217

E-mail kendrickforestfarm@earthlink.net

Private, wholesale, retail. Plants.

Kentucky Division of Forestry-

John P Rhody Nursery

515 John P Rhody Ln,

Gilbertsville KY 42044

Contact Joanna Davidson

Ph 270.362.8331 Fax 270.362.7512

E-mail joanna.davidson@ky.gov

State, retail. Plants.

Kentucky Division of Forestry-

Morgan County Nursery

326 Tree Nursery Rd,

West Liberty KY 41472

Contact Charlie Saunders

Ph 606.743.3511 Fax 606.743.1999

E-mail charlie.saunders@ky.gov

State, wholesale. Plants.

Kettle Moraine Natural

Landscaping

W 996 Birchwood Dr,

Campbellsport WI 53010

Contact Connie Ramthun

Ph 920.533.8939

E-mail brchwood@thesurf.com

Private, wholesale, retail, mail. Seeds.

King Estate I

DBA Lorane Grape Vines

80854 Territorial Rd, Eugene OR 97405

Contact Meliton Martinez

Ph 541.942.9874; 800.884.4441

Fax 541.942.9867

E-mail info@kingestate.com

Private, wholesale. Plants.

King Nursery

6128 E Sandwich Rd,

Big Rock IL 60511

Contact Jim King

Ph 630.554.1171 Fax 630.554.1348

E-mail jk@surfwizard.com

Private, wholesale. Plants. 
Kingfisher Farm

29633 170th Ave, Long Grove IA 52756

Contact Joe Slater

Ph 563.285.5406 E-mail kingfshr@netins.net

Private, wholesale, retail. Plants, Seeds.

Kintigh's Mountain Home Ranch

38865 E Cedar Flat Rd, Springfield OR 97478

Contact Cheryl or Dan Kintigh

Ph 541.746.1842 Fax 541.746.1842

Private, wholesale. Plants.

Klamath Forest Nursery

7680 Happy Hollow Ln, Bonanza OR 97623

Contact Jim Ph 541.545.6432 Fax 541.545.6886

E-mail jdixon@jw-tr.com

Forest Industry. Plants.

Klem's Song Sparrow

13101 E Rye Rd, Avalon WI 53505

Ph 800.553.3715 Fax 608.883.2257

E-mail info@songsparrow.com

Private, mail. Plants.

Kneght's Nurseries

PO Box 241, 1601 Hwy 19 W, Northfield MN 55057

Contact Leif or Deb Knecht

Ph 507.645.5015; 800.924.5015 Fax 507.645.5518

E-mail leif@knechts.net

Private, retail. Plants.

Kobe Nurseries

43624 Co Rd 653, Paw Paw Ml 49079

Contact Paul Kobe Ph 269.657.3094

Private, wholesale. Seeds.

\section{KOLLAR NURSERY}

5200 W Heaps Rd, Pylesville MD 21132

Contact Sharon Kollar

Ph 410.836.0500 E-mail kollars@earthlink.net

Private, wholesale, retail, since 1985. Plants: tree, shrub.

Specializing in container plants native to the eastern US. Contract growing. Please inquire regarding delivery. Smaller quantities of species not listed on our website.

See ad on page 187.

Krueger's Northwoods Nursery

3682 Limberlost Rd, Rhinelander WI 54501

Ph 715.369.3959

Private, wholesale. Plants.

Krueger's Tree Farms

PO Box 32, 21001 NW Dairy Creek Rd,

North Plains OR 97133

Ph 503.647.1000 E-mail sales@kruegertree.com

Private, wholesale, retail. Plants.

\section{Jiffy-7 Forestry}

Rooting Forest Sustainability

Multiple Media Choices for growing your Native plants, Forestry seedlings \& Ornamental plants

- Clean, high quality peat moss

- Media \& container in one

- Always handleable, sizes $10 \mathrm{ml}$ to $405 \mathrm{ml}$.

- Many tray selections

Rooting Above the Competition

Forestry Pellets

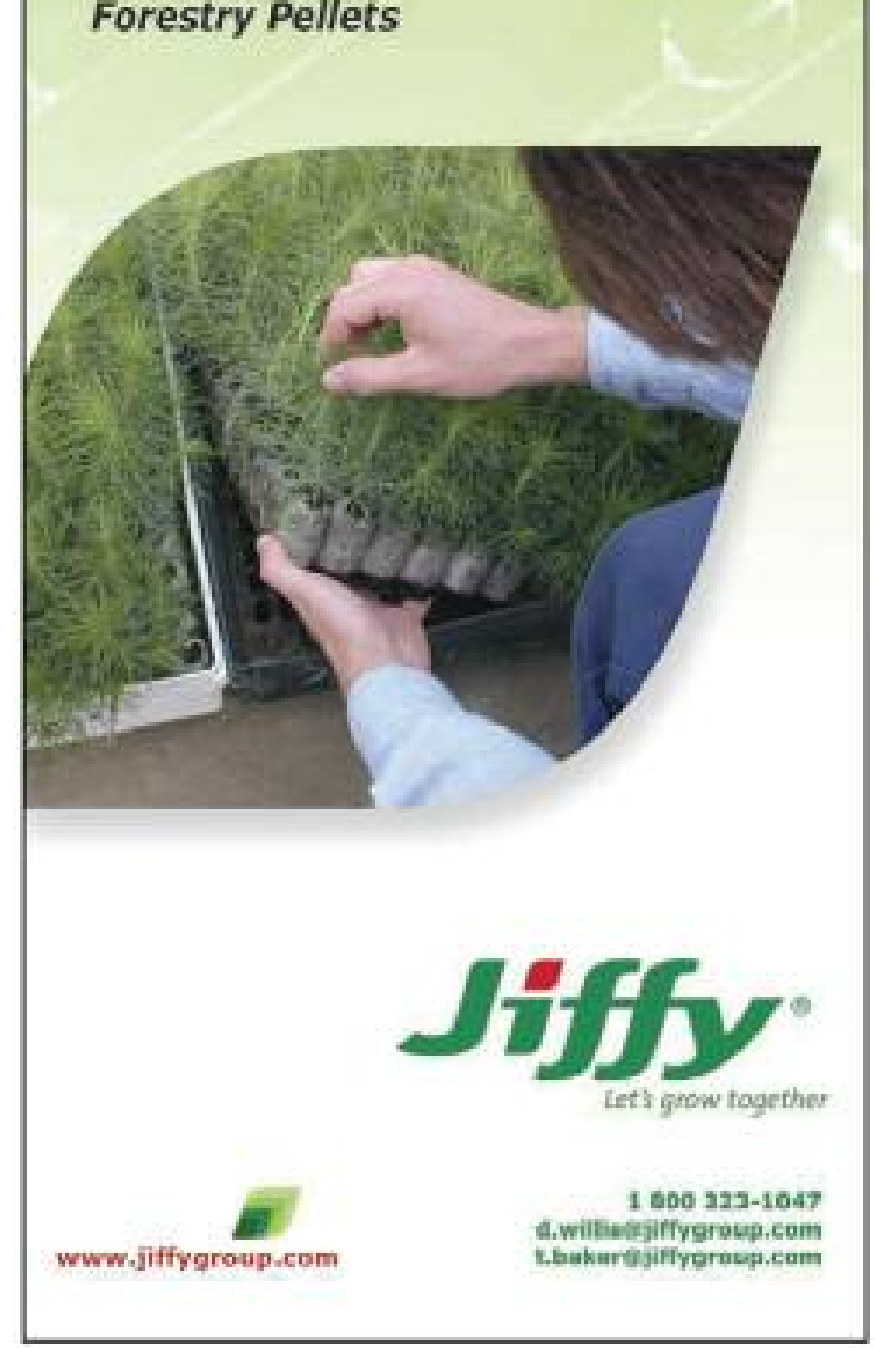

Alse enalable is Ficform

Bonded media

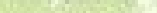




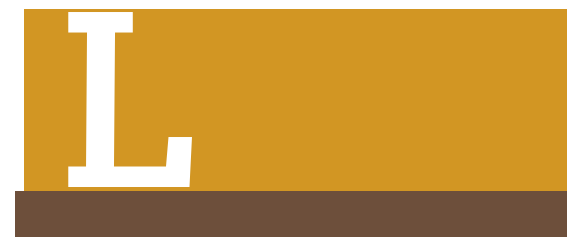

L\&H Seeds Inc

4756 W Hwy 260, Connell WA 99326

Contact Paul Herrman

Ph 509.234.4433 Fax 509.234.0202

E-mail Ihseeds@lhseeds.com

Private, wholesale, retail. Seeds.

Lafayette Home Nursery Inc

9662 TR 50 E, 1 Nursery Ln,

Lafayette IL 61449

Contact David Lahr or Colleen Stevens

Ph 309.995.3311 Fax 309.995.3909

E-mail lafayettehomenursery@winco.net

Private, wholesale. Seeds.

Lake Superior Tree Farms

39425 Hwy US 41, Chassell MI 49916

Contact Stephanie or Bob

Ph 906.523.6200 Fax 906.523.6300

Private, wholesale, retail. Plants.

Lakeshore Garden Centre

Box 92 RR3, Saskatoon SK S7K 3J6

Contact Vic

Ph 306.382.2077; 306.477.0713

Fax 306.382.6433

E-mail vic@lakeshoregardencentre.com

Private, wholesale, retail, mail. Plants.

\section{LAKESHORE TREE FARMS LTD}

960 Valley Rd, Box 92A RR \#3,

Saskatoon SK S7K 3J6

Contact Aaron Krahn

Ph 306.978.3333; 888.995.5253,

cell 306.220.7407 Fax 306.382.6433

E-mail aaron@|shore.com

URL http://www.lakeshoretreefarms.com

Private, wholesale, retail, M-F 8-4:30, since

1936. Plants: $75 \%$ native, $75 \%$ propagated,

$5 \%$ wild collected; tree, shrub.

Large conifer and large-caliper deciduous trees, bareroot and container trees, container shrubs, seedlings and rooted cuttings. Specializing in zone 2 native plants.
THE LAND NURSERY

513 Sharon Rd, 440 N 725 W, West

Lafayette IN 47906

Contact Walter Beineke

Ph 765.463.2994

E-mail wbein2@verizon.net

Private, wholesale, retail, mail, by appt, since 1990. Plants: $500 / y, 85 \%$ native, $90 \%$ propagated, $10 \%$ wild collected; container, bareroot, $B \& B$; tree, shrub.

Supplier of bareroot pine and hardwood seedlings for reforestation, conservation, wildlife enhancement, and mitigation plantings.

I am a retired forestry professor growing native trees and shrubs not found at most nurseries. Seed is collected in northern and central Indiana.

Landmark Seed Company I

Sun Mountain Native Seed

522 W Riverside, Ste 430,

Spokane WA 99201

Contact Orlin Reinbold or RJ

Ph 800.268.0180 Fax 509.835.4969

E-mail orlin@nativeseed.us

Private, wholesale. Seeds.

Landscape Alaska

PO Box 32654, Juneau AK 99803

5372 Commercial Blvd,

Juneau AK 99801

Contact Margaret Tharp or David

Lendrum

Ph 907.790.4916 Fax 907.790.2629

E-mail landscapealaska@gci.net

Private. Plants, Seeds.

Landscape Alternatives

25316 Saint Croix Trail,

Shafer MN 55074-9609

Contact Karl Ruser

Ph 651.257.4460 Fax 651.257.4499

E-mail landscapealt@frontiernet.net

Private, wholesale, retail. Plants.

Larner Seeds

PO Box 407, Bolinas CA 94924

Contact Judith

Ph 415.868.9407 Fax 415.868.2592

E-mail info@larnerseeds.com

Private, wholesale, retail, mail. Seeds.
Las Pilitas Nursery-Escondido

8331 Nelson Way, Escondido CA 92026 Contact Bert

Ph 760.749.5930 Fax 760.749.5932

E-mail bawilson@laspilitas.com

Private, wholesale, retail. Plants.

Las Pilitas Nursery-

Santa Margarita

3232 Las Pilitas Rd,

Santa Margarita CA 93453

Contact Bert

Ph 805.438.5992 Fax 805.438.5993

E-mail bawilson@laspilitas.com

Private, wholesale, retail, mail. Plants.

LAURA'S LANE NURSERY

Box 232, Plainfield WI 54966

Contact Joseph A Wheeler

Ph 715.366.2477 Fax 715.366.8201

E-mail Inur@uniontel.net

URL http://www.lauraslanenursery.com

Private, wholesale, retail, M-Sat 8-4:30,

since 1959. Plants: $90 \%$ native, $95 \%$ propagated, $5 \%$ wild collected. Seeds.

Take advantage of our years of nurserygrowing experience and stock selection. We offer proven varieties to give you the most hardy and reliable stock available. In quantity, at great prices, direct from our nursery for professional growers, foresters, and homeowners. Please call with any questions regarding successful tree planting and care. Or visit our nursery to inspect our seedlings personally.

Lava Nursery Inc

PO Box 370, 5301 Culbertson Dr, Parkdale OR 97041

Ph 541.352.7303 Fax 541.352.7325

E-mail lavanursery@aol.com

Private, wholesale, retail. Plants.

Lawrence Mountain Nurseries

PO Box 185, 24 E Ridge Rd,

Mars Hill ME 04758

Contact Dean

Ph 207.429.9786 Fax 207.429.9786

E-mail marshill1@aol.com

Private, wholesale. Plants.

Lawyer Nursery Inc

7515 Meridian Rd SE,

Olympia WA 98513

Contact Please contact Nick Lawyer or

Sandy Smith at our Montana office (see next listing)

Ph 360.456.1839 Fax 360.438.0344

E-mail bobb@lawyernursery.com

Private, wholesale, retail. Plants. 


\section{KASTE SEED, INC. \\ Growers and Marketers of Native Grass and Wildflower Seeds}

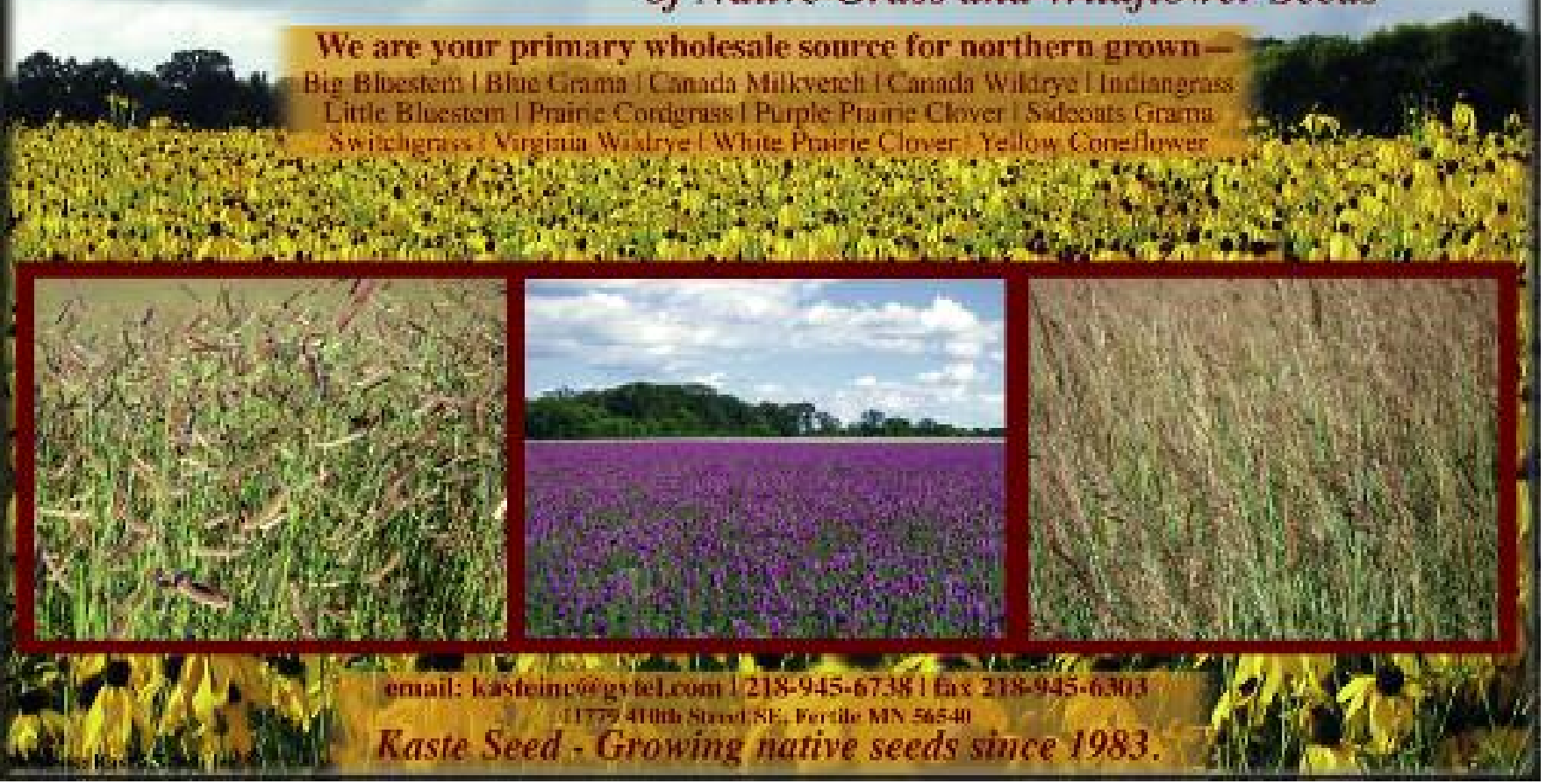

LAWYER NURSERY INC MT

6625 Hwy 200, Plains MT 59859

950 Hwy 200 W, Plains MT 59859

Contact John Lawyer or Michael Johnson

Ph 800.551.9875 Fax 406.826.5700

E-mail trees@lawyernursery.com or seeds@lawyernursery.com URL http://www.lawyernursery.com

Private, wholesale, M-Sat 7:30-5 please call, since 1959. Plants: $20 \%$ native, $100 \%$ propagated; bareroot; tree, shrub. Seeds: $20 \%$ native, $40 \%$ propagated, $60 \%$ wild collected; tree, shrub. Min: $\$ 50$.

We grow 850 species and varieties of woody plants for the wholesale nursery and landscape trade. We are a grower of bareroot nursery stock for the wholesale trade. Our products are grown in open fields for one or more seasons. We also have recently added greenhouse production of conifer and deciduous plugs. We propagate our nursery stock from seed, cuttings, grafts, and tissue culture. We store our crops in modern refrigerated cold storage until they are shipped. We routinely ship to the Midwest, East Coast, and Canada. Lawyer Nursery offers one of the largest selections of field-grown, bareroot, woody plants in the North American wholesale nursery trade. Bareroot material is field grown and shipped without soil. This can lower the costs of shipping compared with container-grown stock, and on a project where every penny counts, bareroot material is an advantage. Bareroot nursery stock is a great choice for nursery liners, rootstocks, Christmas tree plantations, wildlife habitats, and for conservation, mitigation, and reforestation projects. We are consistently looking for perfection in production, and we strive to produce a quality plant at an affordable price. Call Lawyer Nursery to see how our bareroot plant material can fit your project's needs.

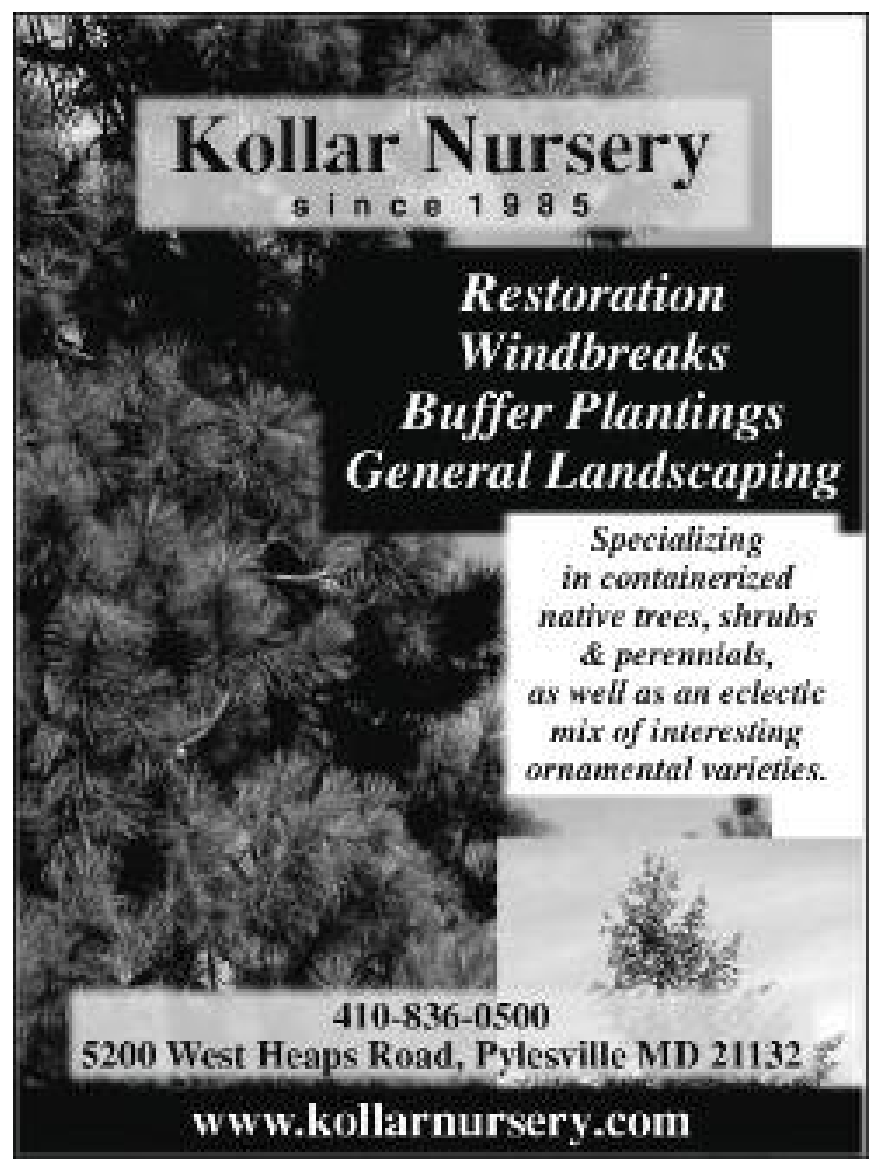


LE Cooke Company

26333 Rd 140, Visalia CA 93292

Ph 559.732.9146 Fax 559.732.3702

Private, wholesale. Plants.

Legacy Land Conservancy

PO Box 2372, Albany OR 97321

Contact Tim Acker

Ph 541.327.3427; 503.871.5472

Fax 541.327.3427

E-mail atwetlands@comcast.net

Private, wholesale, retail, mail. Plants.

Lewis River Reforestation Inc

1203 NW Hayes Rd,

Woodland WA 98674

Contact Robert Moore

Ph 360.225.6357 Fax 360.225.1307

E-mail bobm@llrinc.com

Private, wholesale. Plants.

Lilypons Water Gardens

PO Box 10, 6800 Lily Pons Rd,

Adamstown MD 21717-0010

Contact Jon Sander

Ph 800.999.5459 Fax 800.999.5459

E-mail info@lilypons.com

Private, wholesale, retail, M-Sun 9:30-5:30, since 1917 . Plants: $8 \%$ native, $95 \%$ propagated; container, bareroot, $B \& B$.

Pond installation, consulting, maintenance, cleaning, stocking, repair, water treatment, water test, tours, weddings, festivals.

Lincoln-Oakes Nurseries

3310 University Dr, Bismarck ND 58502

Contact Greg Morgenson

Ph 701.223.8575 Fax 701.223.1291

E-mail lincolnoakes@btinet.net

Private, wholesale. Plants.

Liner Farm Inc

PO Box 701369,

St Cloud FL 34770-1369

Contact David Biggar

Ph 800.330.1484; 407.892 .0038

Fax 407.892.3593

E-mail linerfarminc@aol.com

Private, wholesale. Plants.

Linnaea Nurseries Ltd

3666224 St, Langley BC V2Z 2G7

Contact John Folkerts

Ph 604.533.8281 Fax 604.533.8246

E-mail john@linnaeanurseries.com

Private, wholesale. Plants, Seeds.
Little River Nursery

1508 Crannell Rd, Trinidad CA 95570

Ph 707.268.3069 Fax 707.677.3155

Private, wholesale, retail, mail, contract.

Plants.

Live Oak Nursery

PO Box 2463, Oakdale CA 95361

Ph 209.881.0228; 209.847.3444

Fax 209.847.3444

Private, wholesale, retail. Plants.

Living Desert

12719 Hwy 71 W, Austin TX 78736

Contact Darryl

Ph 512.263.2428

Private, wholesale, retail, mail. Plants.

Lodholz North Star Acres Inc

420 Hwy A, Tomahawk WI 54487

Contact Rich

Ph 800.713.9077

E-mail info@lodholznursery.com

Private, wholesale. Plants.

Lodi Farms Nursery

2880 S Wagner Rd,

Ann Arbor Ml 48103

Contact Dan Riddle

Ph 734.665.5651 Fax 734.665.6393

E-mail jane@lodifarms.com

Private, wholesale, retail. Plants.

Lone Elder Nursery

8051 S Lone Elder Rd,

Canby OR 97013

Ph 503.266.9251 Fax 503.266.6309

Private, wholesale. Plants.

Lonewolf Native Plants

and Herb Farm

Box 2245, Battleford SK SOM OE0

Contact Wanda

Ph 306.937.2192 Fax 306.937.2270

E-mail lonewolf@sasktel.net

Private, wholesale, retail, mail. Plants.

Longfellow's Garden Center

12007 Lookout Trail,

Centertown MO 65023

Contact Alice Longfellow

Ph 573.584.9611 Fax 573.584.9613

E-mail questions@longfellowsgarden.com Private, retail. Plants.
Louisiana Dept of Agriculture

\& Forestry

PO Box 1628, Baton Rouge LA 70821

5825 Florida Blvd,

Baton Rouge LA 70806

Contact Wade Dubea

Ph 225.925.4515 Fax 225.922.1356

State, wholesale. Seeds.

Louisiana Dept of Agriculture \&

Forestry-Columbia Nursery

PO Box 1388, 276 Columbia Nursery

Rd, Columbia LA 71418

Contact Allen Brown

Ph 318.649.7463

E-mail abrown@Idaf.state.la.us

State, wholesale. Seeds.

Louisiana Forest Seed Company

303 Forestry Rd, Lecompte LA 71346

Contact John or Gary

Ph 318.443.5026 Fax 318.487.0316

E-mail info@lfsco.com

Private, wholesale. Seeds.

See ad on page 189.

Louisiana Growers

63279 Lowery Rd, Amite LA 70422

Contact Rick Webb

Ph 985.747.0510 Fax 985.748.5850

E-mail sales@louisianagrowers.com

Private, wholesale. Plants.

Lovelace Seeds Inc

1187 Brownsmill Rd,

Elsberry MO 63343

Contact Judy Lovelace

Ph 573.898.2103 Fax 573.898.2855

E-mail lovelace@inweb.net

Private, wholesale. Seeds.

Lower Marlboro Nursery

PO Box 1013, Dunkirk MD 20754

7011 Flint Hill Rd, Owings MD 20736

Contact Mary Stuart Sierra

Ph 301.812.0808 Fax 301.812.0808

E-mail contact@lowermarlboronursery.com

Private, retail, mail. Plants.

Lowes Creek Tree Farm

S 9475 Lowes Creek Rd,

Eleva WI 54738

Ph 715.878.4166 Fax 715.878.4166

E-mail christmas@lowescreektreefarm.com

Private, wholesale. Plants. 


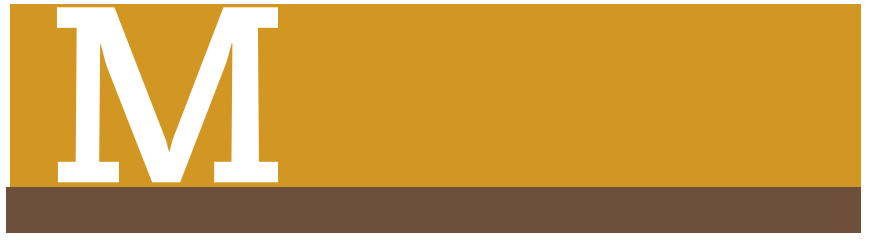

M\&M Native Grass Seed Company

Rt 1 Box 18, Stephensport KY 40170

Ph 270.547.6855

Private. Seeds.

Madrona Nursery

802 37th Ave, Seattle WA 98122

Contact Ann Buscher Ph 206.323.8325 Fax 206.323.8325

E-mail buscherjohn@earthlink.com

Private, wholesale. Plants.

Madrone Nursery

2318 Hilliard Rd, San Marcos TX 78666

Contact Dan Ph 512.353.3944 Fax 512.353.3944

Private, wholesale. Plants.

Mahanoy Valley Nurseries

239 Mahanoy, Duncannon PA 17020

Contact Paul Ph 717.834.3996

Private, wholesale. Plants.

Mahonia Vineyards \& Nursery Inc

2655 Landau St SE, Salem OR 97306

Ph 503.585.8789 Fax 503.363.2358

E-mail ferenc@mahonianursery.com

Private, wholesale, contract. Plants.

Mail-Order Natives

PO Box 9366, Lee FL 32059

Contact Amy Webb

Ph 850.973.6830 Fax 850.973.0585

E-mail www.superiortrees.net

Private, wholesale, retail, mail. Plants.

Makah Tribal Nursery

PO Box 116, Neah Bay WA 98357

Contact Haley McCarty

Ph 360.645.3067 Fax 360.645.2162

E-mail vantilborg@excite.com

Tribal, contract. Plants.

Makani Gardens

1625 W Kuiaha Rd, Ha'iku Maui HI 96708

Contact Gordon

Ph 808.572.6337 Fax 808.572.6337

Private, wholesale. Plants.

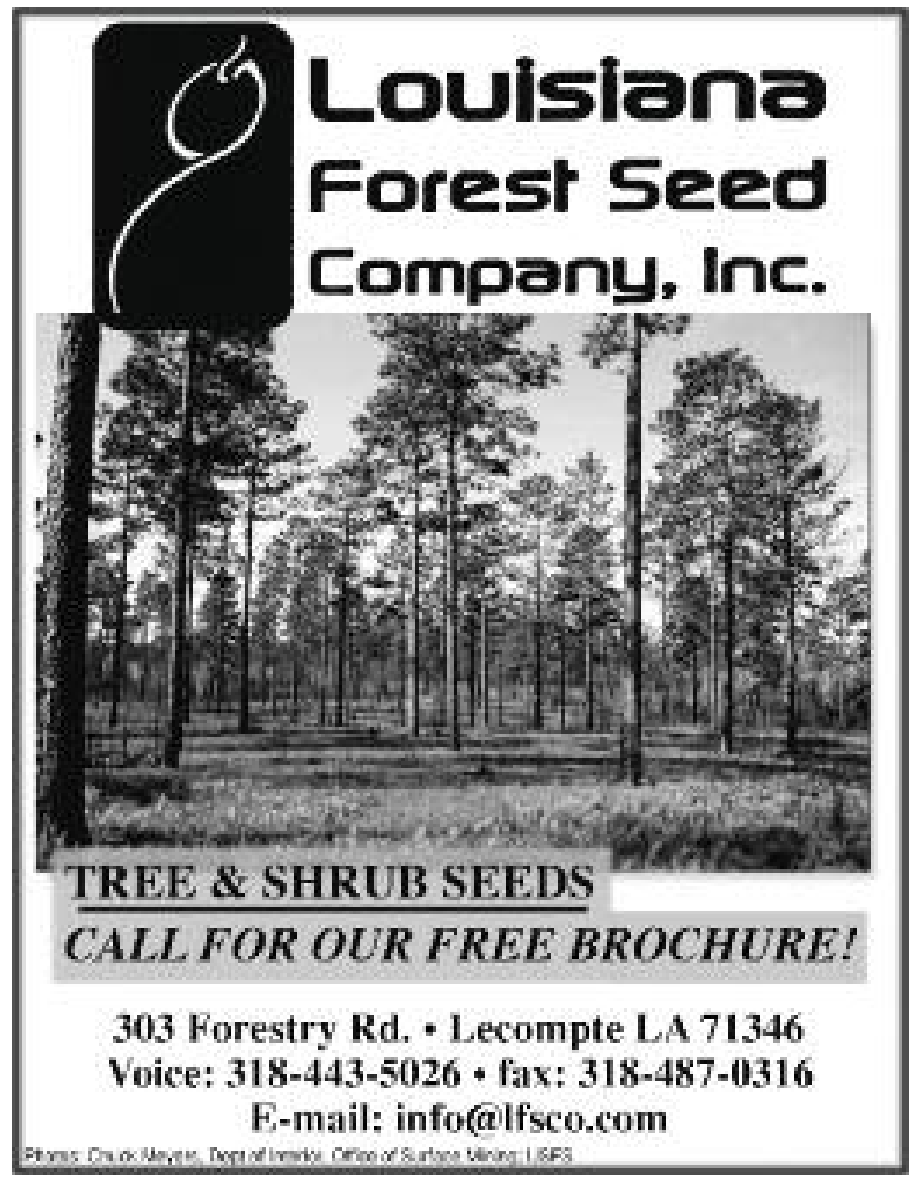

\section{directory key}

NATIVE PLANTS-R-US

123 Main Street, Anytown ID 83843

Contact John Q Public

Ph 800.555.1212 Fax 800.555.1212

BUSINESS TYPE

TYPE

BUSINESS

TYPES OF

PLANTS

Private, wholesale, retail, mail order, M-Sat 8-5

Since 1985. Bareroot, container, seeds: — PRoduct tYPES

$\%$ OF

PRO-

DUCTION

THAT
IS NATIVE

$100 \%$ native, $85 \%$ propagated, $15 \%$ wild collected. OF PLANTS

Min wholesale $\$ 500$, retail none.

s seer

Services: on-line catalog and ordering. \% OF ANNUAL

See ad on page 45- PRODUCTION THAT IS

MINIMUM

ORDER

OTHER We offer more than 600 species of plants native to

SERVICES the northern Rocky Mountains, specializing in locally

collected, source-identified Palouse Prairie plants. Our

staff can help you plan your restoration project, facili-

tate collection and amplification of local seed sources,

and supervise the restoration.

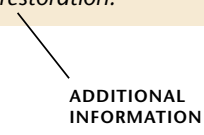


Manzanita Native Plant Nursery 39397 Hwy 94, Boulevard CA 91905 Contact Tim

Ph 619.766.4210

E-mail manzanitanursery@aol.com

Private, wholesale, retail. Plants.

Maple Hill Farms

PO Box 648, William Penn Dr at Airport

Rd, Lewisburg PA 17837-0648

Contact Jeff Craul

Ph 570.524.0791 Fax 570.524.0791

E-mail treetrimmer17837@yahoo.com

Private, wholesale, retail, mail. Plants.

Maple Leaf

450 S 50 E, Ephraim UT 84627

Contact Lloyd

Ph 435.283.4400 Fax 435.283.6872

E-mail maplelf@cut.net

Private, wholesale, retail. Seeds.

Maple Street Natives

2395 Maple St W, Melbourne FL 32904

Contact Sharon or Brent Dolan

Ph 321.729.6857 Fax 321.729.6857

E-mailinfo@maplestreetnatives.com

Private, wholesale, retail. Plants.

Mapleton Nurseries

PO Box 396, 140 Mapleton Rd,

Kingston NJ 08528-0396

Contact David Reid

Ph 609.430.0366; 888.239.7567

Fax 609.430.0367

E-mail mapletonurseries@hotmail.com

Private, wholesale. Plants.

Mark E Gullickson

10990 423rd St SE,

Fertile MN 56540-9272

Contact Mark E Gullickson

Ph 218.945.6894

E-mail mgullic@hotmail.com

Private, wholesale, retail. Seeds.

Marshall Tree Farm

17350 SE 65th St, Morriston FL 32668

Contact Michael Marshall

Ph 800.786.1422 Fax 352.528.3778

E-mailmichael@marshalltrees.com

Private, wholesale. Plants.
Marshland Transplant

Aquatic Nursery

PO Box 1, 116 E Huron St,

Berlin WI 54923

Contact Thomas Traxler Jr

Ph 800.208.2842 Fax 920.361.4200

E-mailmarshland@centurytel.net

Private, wholesale, mail, Internet, contract,

M-F 8-4, since 1984. Plants: $100 \%$ native,

$100 \%$ propagated; container.

Martin Perennial Farms Inc

Rt 2, Box 750, Fort Cobb OK 73038

Ph 800.554.3139 Fax 580.637.2569

Private. Plants.

Maryland Aquatic Nurseries

3427 N Furnace Rd,

Jarrettsville MD 21084

Contact Kelly

Ph 410.557.7615 Fax 410.692.2837

E-mail info@marylandaquatic.com

Private, wholesale, retail. Plants.

Maryland Natives Nursery

PO Box 158, 4812 E Joppa Rd, Perry

Hall MD 21128

Contact Roy List

Ph 410.529.0552 Fax 410.529.3883

E-mailmdn@qis.net

Private, retail, M-F 8-5, since 1998. Plants:

$100 \%$ native, $100 \%$ propagated.

Maryland Dept of Natural

Resources-John S Ayton State

Tree Nursery

3424 Gallagher Rd, Preston MD 21665

Contact Richard Garrett

Ph 800.873.3763 Fax 410.673.2467

E-mail rgarrett@dnr.state.md.us

State, contract. Plants. Seeds.

Mary's Plant Farm \& Landscaping

2410 Lanes Mill Rd, Hamilton $\mathrm{OH}$

45013-9181

Contact Mary Harrison or Sherri Berger

Ph 513.894.0022 Fax 513.892.2053

E-mail marysplantfarm@zoomtown.com

Private, retail, mail, Internet. Plants, Seeds.

Mathisen Tree Farms

PO Box 45, 7288 Grow Rd, Greenville MI 48838

Contact Tom Trechter

Ph 616.754.3200 Fax 616.754.4580

E-mail tom@mathisentreefarms.com

Private, wholesale. Plants.
Matilija Nursery

PO Box 425, Moorpark CA 93021

Ph 805.523.8604

Private, wholesale, retail. Plants.

Matlack Tree Farm

PO Box 67, Minneola FL 34755

11631 Co Rd 561, Claremont FL 34711

Contact Ty Matlack

Ph 352.406.9735 Fax 352.394.2830

Private, wholesale. Plants.

Maughan Seed Company

PO Box 72, 194 W 300 S, Manti UT

84642-0072

Contact Brad Maughan

Ph 435.835.0401 Fax 435.835.0401

E-mail maughanseed@mail.manti.com

Private, wholesale, retail, mail. Seeds.

Maui Native Nursery

68 Mano Dr, Kula HI 96790

Contact Ethan

Ph 808.281.8494 Fax 808.876.1020

E-mail ethanromanchak@mauina

tivenursery.com

Private. Plants.

Mayo Creek Gardens

PO Box 351, 6596 McLean Rd,

Lake Cowichan BC VOR 2G0

Contact Ingeborg Woodsworth

Ph 250.749.6291

E-mail mayocreekgardens@shaw.ca

Private, wholesale, retail. Plants.

McCormick Seeds

PO Box 590, 400 E Ash,

Muleshoe TX 79347

Contact Tim McCormick

Ph 806.272.3156 Fax 806.272.5602

E-mail mcseed54@msn.com

Private, wholesale, retail. Seeds.

McHenry County Nursery Inc I

Glacier Oaks Native Nursery

8216 White Oaks Rd,

Harvard IL 60033-8310

Contact Mary McClelland

Ph 815.482.7404 Fax 815.943.3511

E-mail marytreemcclelland@gmail.com

Private, wholesale, contract. Plants. 
McKeithen Growers Inc

24005 71st Ave E,

Myakka City FL 34251

Contact Eddie McKeithen Ph

941.322.8060 Fax 941.322.2397

E-mail eddie@mckeithen.net

Private, wholesale, retail. Plants.

McNeal Growers

PO Box 371, 105 Twin Creeks Rd, Manchaca TX 78652

Contact Pat McNeal

Ph 512.280.2233 Fax 512.291.5220

E-mail xerxes@io.com

Private, wholesale. Plants.

Meadow Beauty Nursery

5782 Ranches Rd, Lake Worth FL 33463

Contact Carl Terwilliger

Ph 561.601.9673; 561.966.6848

E-mail clwillow@comcast.net

Private, wholesale, retail. Plants.

\section{MEADOWBROOK NURSERY I}

WE-DU NATIVES

2055 Polly Spout Rd,

Marion NC 28752

Contact Jamie Oxley

Ph 828.738.8300

E-mailinfo@we-du.com

URL http://www.we-du.com

Private, wholesale, retail, M-F 9-5 (retail hrs seasonal; Mar-Nov W-Sat 9-5), since 1981.

Plants: $75 \%$ native, $85 \%$ propagated.

We are a wholesale, mail order, and retail nursery with a wide variety of Southeastern native wildflower, trees, shrubs, and other exceptional unusual plants.

Meadowood Nursery

24 Meadowood Dr, Hummelstown PA 17036

Ph 717.566.9875 E-mail meadowoodnursery@comcast.net

Private, wholesale, retail. Plants.

Meeks Farms

168 Flanders Rd, Kite GA 31049

Ph 478.469.3370 Fax 478.469.3150

E-mailsnmek@pineland.net

Private. Plants.
Mellow Marsh Farm

1312 Woody Store Rd,

Siler City NC 27344

Contact Joan McLean

Ph 919.742.1200 Fax 919.742.1280

E-mail plantinfo@mellowmarshfarm.com

Private, wholesale. Plants.

Menzie's Native Nursery

PO Box 9, 10805 N Old Stage Rd,

Weed CA 96094

Contact Robert Menzies

Ph 530.938.4858 Fax 530.938.4858

E-mail menzies@snowcrest.net

Private, wholesale, retail. Plants.

Mesa Garden

PO Box 72, 58 Greer Rd,

Belen NM 87002

Contact Steven Brack

Ph 505.864.3131 Fax 505.864.3124

E-mail cactus@swcp.com

Private, wholesale, retail, mail. Seeds.

Mesozoic Landscapes Inc

7667 Park Ln Rd,

Lake Worth FL 33467-6728

Contact Richard Moyroud

Ph 561.967.2630

E-mailmoyroud@prodigy.net

Private, wholesale. Plants.

Mesquite Valley Growers

8005 E Speedway, Tucson AZ 85710

Contact Kathy Bishop

Ph 520.721.8600 Fax 520.722.2909

E-mail orchidcat@aol.com

Private, retail. Plants.

\section{METHOW NATIVES}

19 Aspen Ln, Winthrop WA 98862

Contact Robert Crandall

Ph 509.996.3562

E-mail methownatives@methownet.

com

Private, wholesale, retail, F 9-4 by appt.

Plants, Seeds.

Methow Natives is a regional native plant nursery. From locally collected seed, we produce a variety of native trees, shrubs, forbs, and grasses for restoration and landscaping projects. We work with small-scale landowners and large organizations, including WDOT, USFS, USFWS, and OCD. Call for availability.
Michigan Dept of Natural

Resources-Wyman State Forest

Nursery

480 N Intake Park Rd,

Manistique MI 49854

Contact Richard Mergener

Ph 906.341.2518 Fax 906.341.8344

E-mail mergenerr@michigan.gov

State, retail. Plants.

Michigan Wildflower Farm

11770 Cutler Rd,

Portland MI 48875-9452

Contact Esther Durnwald

Ph 517.647.6010 Fax 517.647.6072

E-mail wildflowers@voyager.net

Private, mail, M-F 8-5, since 1989. 100\%

native, $10 \%$ propagated. Plants, Seeds.

Mid Atlantic Natives

12506 Susquehanna Trail S, New

Freedom PA 17349

Ph 717.227.0924 Fax 717.227.0653

E-mail customerservice@midatlanticna tives.com

Private, wholesale, retail. Plants.

Middletown Rancheria

PO Box 1035, Middletown CA 95461

Contact Chris Simon

Ph 707.987.3670; 707.987.1109

Fax 707.987.9091

E-mail chris@haypa.com

Tribal, wholesale. Plants.

Midwest Cactus

PO Box 163, New Melle MO 63365

Ph 636.828.5389

Private. Plants.

Miles W Fry \& Son

300 Frysville Rd, Ephrata PA 17522

Contact Morton Fry

Ph 717.354.4501 Fax 717.354.8423

Private, wholesale. Plants.

Milestone Nursery

PO Box 907, 620 State St,

Lyle WA 98635

Contact Modene Miles

Ph 509.365.5222

E-mail milestonenursery@embarqmail.net Private, wholesale, mail, contract, M-F by appt, since 1995. Plants: $100 \%$ native, $100 \%$ propagated; container, bareroot, cuttings. Seeds: $65 \mathrm{lb} / \mathrm{y}, 100 \%$ native, $100 \%$ wild collected. Min: $\$ 100$. 
Millane Nurseries

604 Main St, Cromwell CT 06416-1443

Contact Michael Millane

Ph 860.635.5500 Fax 860.635.3685

E-mailmike@millane.com

Private, wholesale, retail. Plants.

Miller Nursery \& Tree Company

6407 S US Hwy 377,

Stephenville TX 76401

Ph 254.968.2211 Fax 254.968.6903

E-mail sales@millernurseryandtree.com

Private, wholesale. Plants.

Mineland Reclamation Division

801 SW Hwy 169 Ste 2,

Chisholm MN 55719

Contact Dan Jordan

Ph 218.254.7967 Fax 219.254.7973

E-mail dan.jordan@irrrb.org

State. Plants.

Minnesota Native Landscapes

8740 77th St NE, Otsego MN 55362

14088 Hwy 95 NE, Foley MN 56329

Contact Keith Fredrick

Ph 763.295.0010 Fax 763.295.0025

E-mail keith@mnnativelandscapes.com

Private, wholesale, retail, contract. Plants.

Minnesota State Forest

Nurseries-Minnesota Dept of

Natural Resources

13885 State Hwy 64, Ackley MN 56433

Contact Craig Van Sickle

Ph 218.652.2385

State, retail, M-F 8-4:30, since 1929. Plants: $100 \%$ native, $98 \%$ propagated, $2 \%$ wild collected.

Minnesota Valley Garden Center

3232 W 150th St, Shakopee MN 55379

Contact Barb Root

Ph 952.445.9160 Fax 952.445.9161

E-mail barbr@minnesotavalley.com

Private, wholesale, retail. Plants.

Minto Island Growers

PO Box 1004, Salem OR 97308

3394 Brown's Island Rd S,

Salem OR 97302

Contact Chris Jenkins

Ph 503.363.0467 Fax 503.362.5248

E-mail chris@mintogrowers.com

Private, wholesale. Plants, Seeds.
Mississippi State University-

Coastal Research and Extension

Center

PO Box 1639, Picayune MS 39466

1815 Popp's Ferry Rd, Biloxi MS 39532

Contact Patricia Knight

Ph 228.388.4710 ×22

Fax 228.388.1375

E-mail tricia@ra.msstate.edu

State. Plants.

Mississippi State University-

The Crosby Arboretum

PO Box 1639, 370 Ridge Rd, Picayune

MS 39466

Contact Patricia Drackett

Ph 601.799.2311 x22

Fax 601.799.2372

E-mail drackett@ext.msstate.edu

State. Plants.

MISSOURI DEPT OF

CONSERVATION-GEORGE O

WHITE STATE FOREST NURSERY

14027 Shafer Rd, Licking MO 65542

Contact Gregory Hoss

Ph 573.674.3229 x22

Fax 573.674.4047

E-mail greg.hoss@mdc.mo.gov

URL http://www.mdc.mo.gov/forest/

nursery/seedling

State, retail, M-F 8-4:30, since 1937. Plants:

$95 \%$ native, $100 \%$ propagated.

We grow about 70 species of native hardwood trees and shrubs as bareroot seedlings. All from Missouri or Midwest collected seed. We grow about 8 conifer species including 2 native species.

\section{MISSOURI WILDFLOWERS}

\section{NURSERY LLC}

9814 Pleasant Hill Rd, Jefferson City MO 65109-9805

Contact Mervin Wallace

Ph 573.496.3492 Fax 573.496.3003

E-mailmowldflrs@socket.net

URL http://www.mowildflowers.net

Private, wholesale, retail, mail, contract, M-Sat 9-5 Sun 12-5:30, since 1984. Plants: $70,000 / y, 99 \%$ native, $100 \%$ propagated; container; tree, shrub, herbaceous, grass, wetland/aquatic. Seeds: $99 \%$ native, 100\% propagated; shrub, herbaceous, grass.

26th year! Provides contacts for consulting and landscaping. Contract growing. Catalog available.
Mistletoe Carter Wholesale Seed 780 Glen Annie Rd, Goleta CA 93117 Contact Cindy

Ph 805.968.481 Fax 805.968.2242

E-mail sales@mcseeds.com

Private, wholesale. Seeds.

Mobley Greenhouse Inc

1265 Georgia Hwy 133 N,

Moultrie GA 31768

Contact Wayne Bell

Ph 229.985.0321; $1.800 .063 .3450 \times 6$

Fax 229.890.0087

E-mail wbell@interforestry.com

Private, wholesale, retail. Seeds.

Mockingbird Nurseries Inc

1670 Jackson St, Riverside CA 92504

Contact Joni Clayton

Ph 951.780.3571 Fax 951.780.4037

Private, wholesale, retail, contract, M-F 8-4, since 1980 . Plants: $85 \%$ native, $75 \%$ propagated, $25 \%$ wild collected.

Contract growing.

Mohave Joshua Company

PO Box 3222, Kingman AZ 86402

Contact Elno Roundy

Ph 928.757.2818 Fax 928.757.5098

E-mail cleo@citlink.net

Private, wholesale. Seeds.

Mohn Seed Company

3560 265th Ave,

Cottonwood MN 56229

Contact Matthew or Robert

Ph 507.423.6483 Fax 507.423.5552

E-mailmohnseed@mvtvwireless.com

Private. Seeds.

Molpus Timberlands

Management LLC I Joshua

Timberlands Nursery

29650 Comstock Rd, Elberta AL 36530

Contact Sam Campbell

Ph 251.986.5210 Fax 334.986.5211

E-mail scampbell@molpus.com

Private, wholesale. Plants, Seeds. 
MONTANA DEPT OF NATURAL RESOURCES \& CONSERVATIONMONTANA CONSERVATION SEEDLING NURSERY

2705 Spurgin Rd, Missoula MT 59804

Contact John Justin, Seedling Nursery

Manager

Ph 406.542.4244 Fax 406.542.4203

E-mail jjustin@mt.gov

URL http://www.dnrc.state.mt.us/forestry/

nursery

State, retail, mail, Internet, contract, M-F

8-4:30 please call, since 1953 . Plants:

$1,200,000 / y, 90 \%$ native, $100 \%$ propagat-

ed; container, bareroot, cuttings; tree,

shrub, herbaceous, grass.

We produce locally adapted conservation seedlings for Montana and the Northern Rockies, specializing in native Montana seed sources.

Producer of locally adapted, source-identified plants for reforestation, restoration, riparian revegetation, wildlife habitat, and other conservation plantings.

Monterey Bay Nursery

PO Box 1296, Royal Oaks CA 95076

Contact Luen Miller

Ph 831.724.6361 Fax 831.724.8903

E-mail luen@montereybanys.com

Private, wholesale. Plants.

Moon Mountain Wildflowers

PO Box 725,

Carpinteria CA 93014-0725

Ph 805.684.2565 Fax 805.684.2798

E-mail ssseeds@silcom.com

Private, wholesale, retail, mail. Plants.

Moosa Creek Nursery

11760 Betsworth Rd,

Valley Center CA 92082

Ph 760.749.3216 Fax 760.751.0548

E-mail nursery@moosacreek.com

Private, wholesale, contract. Plants.

\section{MORNING SKY GREENERY}

44804 E Hwy 28, Morris MN 56267

Contact Sally Finzel

Ph 320.795.6234 Fax 320.795.6234

E-mail info@morningskygreenery.com

or natives@info.link.net

URL http://www.morningskygreenery.com

Private, wholesale, retail, since 1993. Plants:

$100 \%$ native, $25 \%$ propagated, $75 \%$ wild collected.

Lakescape consultation, contract growing, catalog available.
Moss Greenhouses

269 S 300 E, Jerome ID 83338

Contact Dana Moss

Ph 208.324.1000 Fax 208.324.7391

E-mail dana.moss@mossgreenhouses.com Private, wholesale, retail. Plants.

Mosterman Plant Propagators 43583 Adams Rd, Chilliwack BC V2R 4L1 Contact Theo or Sylvia Mosterman Ph 604.823.4713 Fax 604.823.4749

E-mailmppbc@uniserve.com

Private, wholesale. Plants.

Mostly Natives

PO Box 258, 27235 Hwy 1,

Tomales CA 94971

Contact Walter Earle

Ph 707.878.2009 Fax 707.878.2079

E-mail plants@mostlynatives.com

Private, retail. Plants.

Mount Arbor Nursery

PO Box 129, 201 E Fergusen,

Shenandoah IA 51601

Ph 712.246.4250 Fax 712.246.1841

Private, wholesale, retail. Plants.

Mount Tahoma Nursery

28111 112th Ave E, Graham WA 98338

Contact Rick Lupp

Ph 253.847.9827

E-mail rlupp@aol.com

Private, retail, mail. Plants.

MOUNTAIN STATES WHOLESALE NURSERY

PO Box 2500,

Litchfield Park AZ 85340-2500

10020 West Glendale Ave, Glendale AZ 85307

Contact Dennis Swartzell

Ph 800.840.8509; 623.247.8509

Fax 623.247.6354

E-mail dennis@mswn.com

URL http://www.mswn.com

Private, wholesale, M-F 7-4, since 1969.

Plants: $2,437,756 / y, 80 \%$ native, $100 \%$ propagated; container; tree, shrub, herbaceous, grass.

Mountain States has been a leader in the introduction and production of water-efficient plant materials for more than 39 years, shipping from California to Texas, and north to Idaho.

Leading introducer and producer of waterefficient and native plants adapted to the various elevations of the Southwest.
Mountain Valley Farms 6976 Frix Rd, Cumming GA 30040 Contact Bret Bowlin

Ph 770.886.9334 Fax 770.886 .5170

E-mail mountainvalley@bellsouth.net Private, wholesale. Plants.

Mountain West Seed Company Inc

19 N 100 W, Ephraim UT 84627

Contact Jeremy

Ph 435.283.4704 Fax 435.283.4704

E-mailmtnwseed@cut.net

Private, wholesale. Seeds.

Mourning Cloak Ranch \&

Botanical Gardens

22101 Old Town Rd,

Tehachapi CA 93561

Ph 805.822.1661 Fax 805.822.5062

E-mail mcranch@lightspeed.net

Private, retail. Plants.

MsK Rare and Native Plant

Nursery

20312 15th Ave NW,

Shoreline WA 98177-2166

Contact Sarah Baker

Ph 206.546.1281

E-mail plants@msknursery.com

Private, retail. Plants.

Munchkin Nursery and

Gardens LLC

323 Woodside Dr NW,

Depauw IN 47115-9039

Contact Gene Bush

Ph 812.633.4858 Fax 812.633.4858

E-mail genebush@munchkinnursery.com

Private, retail. Plants.

Murphy's Walnut Hill Nursery Inc

1925 SE 82nd St, Runnells IA 50237

Contact Jim Murphy

Ph 515.262.6037

E-mail jmurtree@aol.com

Private, wholesale, retail. Plants.

Murray Nurseries Ltd

3140 W 57th Ave,

Vancouver BC V6N 3X6

Contact Brian, Heather, or Genevieve

Ph 604.261.2151 Fax 604.266.8514

E-mail murraynurseries@shaw.ca

Private, retail. Plants. 
Musser Forests Inc

PO Box 340, 1880 Rt 119 Hwy N, Indiana PA 15701

Contact Chuck Flinn

Ph 724.465.5686

E-mailinfo@musserforests.com

Private, wholesale. Seeds.

Myers Cove Nursery Inc

3876 Myers Cove Rd, McMinnville TN 37110

Contact Andy or Betty

Ph 931.668.3155 Fax 931.668.3207

E-mail myerscove@blomand.net

Private, wholesale. Plants.

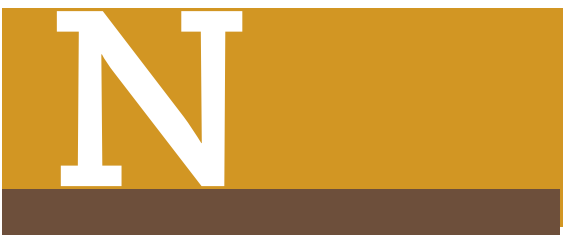

NASHVILLE NATIVES LLC

7443 Liberty Rd, Fairview TN 37062

Contact Andy Sudbrock

Ph 615.799.8719

E-mailinfo@nashvillenatives.com URL http://www.nashvillenatives.com Private, wholesale, by appt, since 2003. Plants, Seeds.

Large selection of TN native plants for ecological restoration and native landscapes. We specialize in restoring and managing native plant communities. Seeding, planting, invasive removal.

Licensed growers of American Beauties Native Plants. Native plants for restoration, parks, and landscaping. Custom seeding, planting, and land management. Wholesale growers since 2003.

Natchez Trace Gardens

1113 S Huntington St,

Kosciusko MS 39090

Contact Mark

Ph 662.289.4979; 662.289.2221

Fax 662.289.1445

Private, wholesale. Plants.

National Tropical Botanical

Garden

3530 Papalina Rd, Kalaheo HI 96741

Ph 808.742.1011 Fax 808.332.9765

Private. Plants.
Native American Seed

3791 N US Hwy 377, 4111 US Hwy

377, Junction TX 76849

Contact Jan Neiman

Ph 800.728.4043 Fax 800.728.3943

E-mailinfo@seedsource.com

Private, wholesale, mail, Internet. Seeds.

Native and Uncommon Plants

4157 Ortega Blvd, Jacksonville FL

32210-4421

Contact Leslie Pierpont

Ph 904.388.9851 Fax 904.388.9851

E-mail lespierpont@mac.com

Private, retail. Plants.

Native and Xeric Plants Inc

8625 Bill Burns Rd, Emmett ID 83617

Contact Jennifer Swenson or

Stew Churchwell

Ph 208.365.4331

E-mail jenn@nativeandxericplants.com Plants.

Native Connections

17080 Hoshel Rd,

Three Rivers MI 49093

Contact Jerry Stewart

Ph 269.580.4766; 269.580.4765

Fax 269.273.1367

E-mail jerry@nativeconnections.net

Private, wholesale, retail. Seeds.

Native Forest Nursery

11306 Hwy 411 S,

Chatsworth GA 30705

Contact Paul Ensminger

Ph 706.483.3397 Fax 706.334.2272

E-mail ensming@comcast.net

Private, wholesale. Plants.

Native Gardens

5737 Fisher Ln, Greenback TN 37742

Contact Ed or Meredith

Ph 865.856.0220 Fax 865.856.0220

E-mailmclebsch@earthlink.net

Private, wholesale, mail. Plants.

Native Ground

PO Box 6045, Helena MT 59604

3005 Airport Rd, Helena MT 59601

Contact Corey Baker

Ph 406.461.5593

E-mailinfo.nativeground@gmail.com Plants.
Native Grounds Nursery

37545 Hwy 228, Brownsville OR 97327

Contact Mike or Keli

Ph 541.466.3561 Fax 541.466.3500

E-mail keli@nativegroundsnursery.com

Private, wholesale. Plants.

Native Habitat Nursery

14140 SE Palmer Creek Rd,

Dayton OR 97114

Contact Spencer

Ph 503.864.4800 Fax 503.864.2489

Private, wholesale, retail. Plants.

Native Haunts

317 Mountain Rd, Alfred ME 04002

Contact Shawn Jalbert

Ph 207.490.0849

E-mail nativehaunts@gmail.com

Private, wholesale, retail. Plants, Seeds.

Native Here Nursery

101 Golf Course Rd, Berkeley CA 94708

Ph 510.549.0211

E-mail Charlie@pacbell.net

Private, wholesale, retail, F 9-12 Sat 10-1, since 1995. Plants: $100 \%$ native, $100 \%$

propagated.

Native Landscapes Garden Center 991 Rt 22, Pawling NY 12564

Contact Peter Muroski

Ph 845.855.7050 Fax 845.855.7016

Private, wholesale, retail. Plants.

Native Landscapes Inc

PO Box 4012, 810 S Main St Unit A,

Hailey ID 82333

Contact James Gillespie

Ph 208.578.2200

E-mail info@coolnativelandscapes.com Private. Plants.

Native Nurseries of Tallahassee Inc 1661 Centerville Rd,

Tallahassee FL 32308

Contact Donna or Norma

Ph 850.386.8882 Fax 850.386.2747

E-mail dlegare@comcast.net

Private, retail. Plants, Seeds.

Native Nursery

17025 S Golden Rd, Golden CO 80401

Contact Tom

Ph 303.278.3750 Fax 303.278.1127

Private, retail. Plants. 
Native Nursery LLC

PO Box 806, 1267 Naalae Rd,

Kula HI 96790

Contact Ethan Romanchak

Ph 808.878.8276 Fax 808.876.1020

E-mailinfo@mauinativenursery.com

Private, wholesale. Plants.

Native Oak Nursery Supply

Las Vegas NV 89123

Ph 702.263.9198

Plants.

Native Ornamentals

PO Box 997, 110 S 6th St,

Mertzon TX 76941

Contact Steve or Valerie

Ph 325.835.2021

E-mail texican@zipnet.us

Private, wholesale, retail. Plants.

Native Plant Farm \& Tree Movers

5005 Old US Hwy 395 N,

Washoe Valley NV 89704

Contact Pat

Ph 775.691 .1490

E-mail nativeplantfarm@softcom.net

Private, wholesale, retail. Plants, Seeds.

Native Plant Growers

PO Box 206, Terra Ceia FL 34250

111 Palm View Rd,

Palm Meadow FL 34221

Contact Sandy Mazer

Ph 941.723.5091 Fax 941.723.5075

E-mail natplant@version.net

Private, wholesale. Plants.

The Native Plant Nursery

PO Box 7841, Ann Arbor MI 48107

Contact Greg Vaclavek

Ph 734.677.3260 Fax 734.677.5860

E-mail plants@nativeplant.com

Private, retail. Plants, Seeds.

Native Plant Restoration Inc

3208 Bearspaw Dr NW,

Calgary AB T2L 1T2

Contact Pat or Al

Ph 403.282.6516 Fax 403.282.6516

E-mail fedkenhp@telus.net

Private, wholesale, retail. Plants, Seeds.
Native Plant Source

318 Misty Crescent, 1098 Wurster PI,

Kitchener ON N2B 3V5

Contact Jeff Thompson

Ph 519.748.2298

E-mail info@nativeplantsource.com

Private, retail, contract. Seeds.

Native Plants in Claremont

4965 Westney Rd,

Pickering/Claremont ON L1Y 1A2

Contact Karen Abrahams

Ph 905.649.8176

E-mail karen@nativeplants.ca

Private, wholesale, retail. Plants.

Native Revival Nursery

2600 Mar Vista Dr, Aptos CA 95003

Contact Erin O'Doherty

Ph 831.684.1811 Fax 831.684.1811

E-mail nativerevival@sbcglobal.net

Private, wholesale, retail, contract. Plants.

NATIVE SEED FOUNDATION

HCR 62 Box 332A, 7312 Perkins Lake

Rd, Moyie Springs ID 83845

Contact Ben or David Ronniger

Ph 208.267.1477 Fax 208.267.3265

E-mailinfo@nativeseedfoundation.com

or ben@nativeseedfoundation.com

URL http://www.nativeseedfoundation

.com

Private, wholesale, 7-5, since 1977. Seeds:

$100 \%$ native, $100 \%$ wild collected. Min: $1 \mathrm{oz}$.

We are experienced collectors and processors of more than 30 species of native seeds, princi-

pally wildlife browse, ornamental, erosion con-

trol, and reclamation. We supply the larger

seed companies and ship worldwide.

Native Seeds

220 E 6th St, Tucson AZ 85705

3061 N Campbell Ave,

Tucson AZ 85719

Contact Vivian McKinnon

Ph 520.622.5561 Fax 520.622.5591

Private, retail. Seeds.

Native Seeds Inc

14590 Triadelphia Mill Rd,

Dayton MD 21036

Ph 301.596.9818 Fax 301.854.3195

E-mail saund10449@aol.com

Wholesale, retail, mail, contract. Seeds.
Native Sod Solutions

PO Box 1115, Driggs ID 83422

Contact Tim Watters

Ph 877.444.6996 Fax 208.247.0941

E-mail tim@nativesodsolutions

Private, wholesale. Plants.

Native Sons Inc

379 W El Campo Rd,

Arroyo Grande CA 93420

Contact Jennifer Scarano or

Jimmy Alcantar

Ph 805.481.5996 Fax 805.489.1991

E-mail jscarano@nativeson.com

Private, wholesale. Plants.

Native Springs Nursery

PO Box 4071, Yankeehill CA 95965

Contact Debra Fau

Ph 530.514.8578

E-mail nativespringsnursery@yahoo.com Contract. Plants.

Native Sun Home \& Garden

Nursery

1047 S Hwy 80, Benson AZ 85602

Contact Cynthia Speir

Ph 520.586.4525

E-mail cindy@native.sun.net

Private, retail. Plants.

Native Technologies Inc

6466 NW 5th Way,

Fort Lauderdale FL 33309

Contact Rob Miller

Ph 954.596.2411; 954.523.5236

Fax 954.462.7011

E-mail inquiry@nativetechnologiesinc

Private, contract. Plants.

Native Texas Nursery

16019 Milo Rd, Austin TX 78725

Contact Jarred Pyka

Ph 512.276.9801 Fax 512.276.9820

E-mail sales@nativetx.com

Private, wholesale. Seeds.

Native Tree Farm

3302 Primrose (mail), 9201 Hwy 29 E,

Georgetown TX 78628

Ph 512.630.3069

Private, wholesale, retail. Plants. 
Native Tree Nursery Inc

17250 SW 232 St, Goulds FL 33170

Contact Hugh Forthman Jr

Ph 305.247.4499 Fax 305.247.4471

E-mail sales@nativetreenursery.com

Private, wholesale. Plants.

\section{THE NATIVES INC}

2929 JB Carter Rd, Davenport FL 33837

Contact Sarah Kiefer

Ph 863.422.6664

E-mail natives@gate.net

URL http://www.thenatives.net

Private, wholesale, retail, M-F 7:30-4, since

1983. Plants, Seeds.

Landscape quality plants. Native ornamental grasses. Sandhill and scrub species. Wetland trees, shrubs, and herbaceous wildflowers. Tubelings to 15 gallons. Upland restoration design and implementation.

\section{NATIVES OF TEXAS}

4256 Medina Hwy,

Kerrville TX 78028-8774

Contact David Winningham

Ph 830.896.2169 Fax 830.257.3322

E-mail dwinningham@mac.com

URL http://www.nativesoftexas.com

Private, wholesale, retail, contract, $\mathrm{M}-\mathrm{Sat}$

9-4 Sun 11-4, since 1995. Plants: 10,000/y,

$99 \%$ native, $50 \%$ propagated, $5 \%$ wild col-

lected; container, cuttings; tree, shrub,

herbaceous, grass, wetland/aquatic. Seeds: 5

$\mathrm{lb} / \mathrm{y}, 100 \%$ native, $90 \%$ propagated, $10 \%$

wild collected; tree, shrub, herbaceous,

grass.

Encourage use of local natives. Our specialty trees are madrones and big tooth maples. We are primarily organic and foster the use of xeriscape plants.

We are a unique regional source of hard-tofind plants of the Texas hill country, especially rare madrones, maples, and witch hazel.

\section{NATIVESWEST NURSERY}

\section{\& ECO-SERVICES LLC}

155 Falcon Ridge, Kooskia ID 83539

Contact Galahad or Kahne Blyth

Ph 208.926.7707 Fax 208.926.7707

E-mail kahneblyth@wildblue.net URL http://www.nativeswest.com Private, wholesale, M-F 9-5:30 Sat 9-4:30 Feb-Nov, since 2000. Plants: $1000 / y, 80 \%$ native, $20 \%$ propagated, $10 \%$ wild collected; container, $B \& B$; tree, shrub, grass, wetland/aquatic.

Native landscape installation and maintenance, custom seed mix, private land development consulting, plant identification surveys, large tree planting and moving.

Our native product is grown in central Idaho therefore is suitable for colder, higher elevation sites. We complement our hard-working native product with fruits and berries to encourage an interactive edible landscape. We grow and install. As growers and installation specialists, we consult with private landowner developers, including designing custom seed mixes, plant surveying, and planting recommendation lists and plans.

See ad on page 197.

NATS NURSERY LTD

24555 32nd Ave, Langley BC V2Z 2J5

Contact Rod Nataros

Ph 604.530.9300 Fax 604.530.9500

E-mail rod@natsnursery.com

URL http://www.natsnursery.com

Private, wholesale, M-F 8-4:30, since 1989.

Plants: $2,000,000 / y, 90 \%$ native, $100 \%$ propagated; container, $B \& B$, cuttings; tree, shrub, herbaceous, grass, wetland/aquatic.

Specialized growers and wholesalers of Pacific Northwest native plants, green roof and living wall plants, hardy ferns, and ground covers. LiveRoof Modular Green Roof System Affiliate Growers. Site-specific plants and contract growing available. Let our skilled team assist you.

THE NATURAL GARDEN INC

38 W 443 Hwy 64, St Charles IL 60175

Contact Jan Sorensen

$\mathrm{Ph} 630.584 .0150 \times 227$

Fax 630.584.0185

E-mail gardeninfo@thenaturalgarden inc.com

URL http://www.thenaturalgardeninc.com Private, wholesale, retail, mail, M-Sat 9-5, since 1953. Plants: $1,000,000 / y, 65 \%$ native, $95 \%$ propagated; container; shrub, herbaceous, grass, wetland/aquatic. Seeds: $65 \%$ native, $95 \%$ propagated; shrub, herbaceous, grass. Min: 1/8 oz.

We specialize in seed propagation of northern Illinois native forbs and grasses. Plants are available in 2.5", 4.5" \& \#1 containers. Limited seed available.

The Natural Gardener

Garden Store

4376 W 10th Ave,

Vancouver BC V6R 2H7

Contact Bob Tuckey

Ph 604.224.2207 Fax 604.224.2216

E-mailinfo@thenatural-gardener.com

Private, retail, M-Sat 10-6 Sun 12-5, since

2003. Plants, Seeds.
Natural Habitat Gardens

RR 1 S15A C35, Sorrento BC V0E 2W0 Contact Trish Wallensteen

Ph 250.835.2221

E-mail kevin\&trish@telus.net

Private, retail. Plants.

Natural Landscapes Nursery

$354 \mathrm{~N}$ Jennersville Rd,

West Grove PA 19390

Contact Bethany or Jim Plyler

Ph 610.869.3788 Fax 610.869.3788

E-mail naturallands@gmail.com

Private, wholesale. Plants.

Natural Legacy Seed

RR 2 C1 Laird, Armstrong BC VOE 1BO

Contact Graham Ware

Private, wholesale. Seeds.

Natural Resource Native

Plant Nursery

2466 Roome Rd, Duncan BC V9L 4L2

Contact Richard Oud

Ph 250.748.0684

E-mail r_oud@shaw.ca

Plants, Seeds.

Natural Shore Technologies Inc 6275 Pagenkopf Rd, 5300 Hwy 12, Maple Plain MN 55359

Contact Rob Langer

Ph 612.703.7581

E-mail info@naturalshore.com

Private, wholesale, retail, contract. Plants.

Naturally Native Nursery

13737 State Rt 582 (Middleton Pike),

Bowling Green $\mathrm{OH} 43402$

Contact Jan Hunter

Ph 419.833.2020

Private, wholesale, retail, mail. Plants.

Nature By Design

300 Calvert Ave, Alexandria VA 22301 Contact Randee Wilson

Ph 703.683.GROW Fax 703.519.GROW

E-mail plantfolks@nature-by-design.com Private, retail. Plants, Seeds. 
The Nature ConservancyChamplain Valley Native Plant Restoration Nursery

115 Main Rd, West Haven VT 05743

Ward Ln, Whitehall NY 12887

Contact Murray McHugh

Ph 802.265.8645 Fax 802.265.4823

E-mailmmchugh@tnc.org

Private, wholesale, retail. Plants.

Nature Hills Nursery Inc

9910 N 48th St, Ste 200,

Omaha NE 68152

Contact Jeff Dinslage

$\mathrm{Ph} 402.934 .8116 \times 101$

Fax 402.991 .0778

E-mail info@naturehills.com

Private, wholesale, retail. Plants.

Nature's Acres I Minnesota

Native Landscapes

14088 Hwy 95 NE, Foley MN 56329

Contact Joe Schaffer or Steve

Ph 320.968.4222 Fax 320.968.4223

E-mail natacres@ecenet.com

Private, wholesale, retail. Plants, Seeds.
NATURE'S ENHANCEMENT INC

2980 Eastside Hwy,

Stevensville MT 59870

Contact Julie Monk

Ph 406.777.3560 Fax 406.777.3560

E-mail

natures_enhancement_inc@msn.com

URL http://www.naturesenhancement inc.com

Private, wholesale, retail, contract, $\mathrm{M}-\mathrm{F}$ 8-4:30, since 1995. Plants: $150,000 / y, 90 \%$ native, $100 \%$ propagated; container, $B \& B$, cuttings; tree, shrub, herbaceous, grass, wetland/aquatic.

A wholesale grower on 35 acres producing hundreds of thousands of trees, shrubs, wildflowers, and grasses annually. Ability to service large projects with plant sizes ranging from $a$ 1-gallon container to larger caliper $B \& B$ trees. Our complete landscape and restoration project divisions offer full services including sitespecific consulting, custom design, installation, irrigation, and contract growing. Delivery services are also available.
Nature's Garden Seed Company PO Box 32105, 3651 Shelbourne St, Victoria BC V8P $5 \mathrm{~S} 2$

Ph 250.595.2062

E-mailmail@naturesgardenseed.com Private, wholesale, since 1994. Seeds: herbaceous.

Nature's Image Inc 20472 Crescent Bay Dr, Lake Forest CA 92630

Ph 949.454.1225 Fax 949.454.1215 Plants.

Naturescapes Nursery 1674 N Bluff Rd, Collinsville IL 62234

Contact Nancee Kruescheck Ph 618.344.8841 Fax 618.344.8841

E-mail plants57@aol.com

Private, wholesale, retail. Plants.

Navajo Forestry Nursery

PO Box 230, Fort Defiance AZ 86504

Contact AK Arbab

Ph 520.729.4007; 602.729.4235

Fax 520.729.4225

Tribal. Plants.

\section{nativeswest uc}

nursery \& eco-services

\section{6}

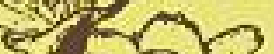

www.nativeswest.com

\section{"Experience The Quality"}

New! Retail Garden Center (Closed Mondays)

\section{Trees \& Shrubs:}

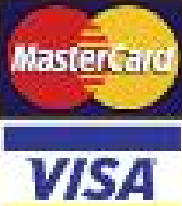

DISCOVER

mivoes

\section{Fruit/Flowering/Shade/Large B\&B}

Edible Landscape Plants \& Colorful Annuals

Native Plant Growers

Sprinkler Installation

Eco-Wise Landscaping

Land Restoration Services/Consulting

Wholesale/Retail (ID lic \#rce-28143): 4253 Hwy 12 Kamiah, ID 83536 
Needlefast Evergreens Inc

4075 W Hansen Rd,

Ludington MI 49431

Contact James Nickelson

Ph 231.843.8524 Fax 231.843.1887

E-mail nickel@needlefastevergreens.com

Private, wholesale, retail, mail, Internet, $\mathrm{M}-\mathrm{F}$

8-5 Sat 8-noon, since 1957. Plants:

4,000,000/y, 90\% native; bareroot. Seeds:

$1000 \mathrm{lb} / \mathrm{y}, 100 \%$ native. Min: $1 \mathrm{~kg}$.

Nevada Division of Forestry-

Las Vegas Nursery

9600 Tule Springs Rd,

Las Vegas NV 89131

Contact Sarah Haymond or Sarah Earle

Ph 702.486.5411 Fax 702.486.5449

State, wholesale, retail, contract. Plants.

Nevada Division of Forestry-

Washoe Nursery

885 Eastlake Blvd,

Carson City NV 89704

Contact Rick Vigen

Ph 775.849.0213 Fax 775.849.2058

State, wholesale, retail, contract. Plants.

New England Environmental

Services

155 Jerry Daniels Rd,

Marlborough CT 06477

Contact Richard Snarsski

Ph 860.295.1022; 860.918.1976

Fax 860.295.1022

Private, wholesale. Plants.

NEW ENGLAND WETLAND

PLANTS

820 West St, Amherst MA 01002

Contact Dave Anderson

Ph 413.548.8000 Fax 413.549.4000

E-mail info@newp.com

URL http://www.newp.com

Private, wholesale, M-F 9-5, since 1996.

Plants: $95 \%$ native. Seeds: tree.

Local ecotype native plants for use in wetland restoration, and other more upland species for naturalistic landscaping. Also erosion control fabrics and native seed mixes.
NEW ENGLAND WILD FLOWER

SOCIETY

180 Hemenway Rd,

Framingham MA 01701

Contact Nicci Cataldo

Ph 508.877.3658 ×3601

Fax 508.877.3658

E-mail nursery@newenglandwild.org or hort@newfs.org

URL http://www.newenglandwild.org

Private, wholesale, retail, mail, contract, Apr

18-Aug 31 M-Sun 9-7; Sep 1-Oct 15

M-Sun 9-5, since 1900. Plants: $100 \%$

native, $100 \%$ propagated; container. Seeds:

$100 \%$ native; tree, shrub, herbaceous, grass.

More than 500 native species, many rare; proceeds support conservation. Contract growing, liner plants, More than 300 native plant education programs. An amazing selection of nursery-propagated natives, with all purchases supporting plant conservation. Become a member to support the environment, obtain free admission to Garden in the Woods, and for discounts on plants, books, and courses.

See ad on page 199.

New Hampshire State

Forest Nursery

405 Daniel Webster Hwy,

Boscawen NH 03303

Contact Daniel DeHart

Ph 603.796.2323 Fax 603.271.6488

E-mail ddehart@dred.state.nh.us

State, wholesale. Seeds.

New Jersey Division of Parks and

Forestry-Forest Tree Nursery

370 E Veterans Hwy,

Jackson NJ 08527-0396

Contact Joe Battersby

Ph 732.928.0029 Fax 732.928.4925

E-mail njforestnursery@optonline.net

State, retail, M-F 7:30-4. Plants.

Provides bareroot tree and shrub seedlings.

New Life Nursery Inc

3720 64th St, Holland MI 49423

Contact Steve Genzink

Ph 269.857.1209 Fax 269.857.1770

E-mail sgenzink@newlifenursery.com

Private, wholesale. Seeds.
New Mexico Forestry Division PO Box 1948, 1220 S St Francis Dr, Santa Fe NM 87504-1948

Contact Paul Lyons

Ph 505.476.3334 Fax 505.476.3330

E-mail wlyons@state.nm.us

State. Plants.

New Mexico State University-

Mora Research Center

PO Box 359, State Hwy 518,

Mora NM 87732

Contact John T Harrington

Ph 575.387.2319 x11

Fax 575.387.9012

E-mail joharrin@nmsu.edu

State, no public sales. Plants.

New Moon Nursery LLC

975 Barretts Run Rd,

Bridgeton NJ 08302

Contact James or Kimberly Brown

Ph 888.998.1951 Fax 888.998.1952

E-mail info@newmoonnursery.com

Private, wholesale. Plants.

See ad on page 199.

New World Botanical

2701 Lone Star Parkway,

Montgomery TX 77356

Ph 936.689.8751 Fax 936.582.6462

Private, wholesale, retail. Plants.

New York State Dept of

Environmental Conservation-

Saratoga Tree Nursery

2369 Rt 50,

Saratoga Springs NY 12866

Contact David J Lee

Ph 518.581.1439 Fax 518.581.8017

E-mail djlee@gw.dec.state.ny.us

State, retail. Plants.

Newaygo Conservation

District Nursery

1725 E 72nd St, Newaygo MI 49337

Contact Louise Brock

Ph 231.652.7493 Fax 231.652.4776

State, wholesale, retail. Seeds.

Newpage Corporation

PO Box 1008, 7100 Co Rd 426,

Escanaba MI 49829

Contact Mary Jo Carlson

Ph $906.786 .1660 \times 2170$

Fax 906.789 .3253

Forest Industry. Plants. 
Niche Gardens

1111 Dawson Rd, Chapel Hill NC 27516

Contact Meg Ph 919.967.0078 Fax 919.967.4026

E-mailmail@nichegardens.com

Private, retail, mail, Internet, M-Sun 9-5, since 1986. Plants: $60 \%$

native, $100 \%$ propagated; container.

Retail and mail order; specializing in nursery-propagated natives and wildflowers; high-quality shipping year-round, catalog available.

Nicholls Gardens

4724 Angus Dr, Gainesville VA 20155

Ph 703.754.9623 Fax 703.754.9623

E-mail nichollsgardens@juno.com

Private, retail, mail, Internet. Plants.

Nick's Nursery Inc

PO Box 2761, 6675 SE Ave, Homosassa Springs FL 34447

Ph 352.628.3098 Fax 352.628.7515

Wholesale, retail. Plants.

Nighthawk Natives Nursery \& Wildlands

Restoration

2944 N Castro Ave, Tucson AZ 85705

Contact Bernadette jilka or

Gary Maskarinec

Ph 520.882.0969 Fax 520.882.0969

E-mail garyberni@aol.com

Private, wholesale, retail, mail, contract. M-Sat 8-5, since 1982.

Plants, Seeds.

Nolin River Nut Tree Nursery

797 Port Wooden Rd, Upton KY 42784

Contact John or Lisa Brittain Ph 270.369.8551

E-mail john.brittain@windstream.net

Private, retail, mail. Plants.

Norfarm Seeds Inc

31154 430th Ave, Roseau MN 56751-8413

Contact Don Berry Ph 218.463.2119 Fax 218.463.3124

Private, wholesale, retail. Seeds.

Norfarm Seeds Inc

104 Minnesota Ave, Bemidji MN 56601

Contact Dennis Montgomery

Ph 218.751.8617 Fax 218.751.0485

Private, wholesale, retail. Seeds.

Norman's Native Plants Plus

2150 US 27 N, Avon Park FL 33825

Fax 863.453.6303

E-mail normansnativeplants@yahoo.com

Wholesale, contract. Plants.
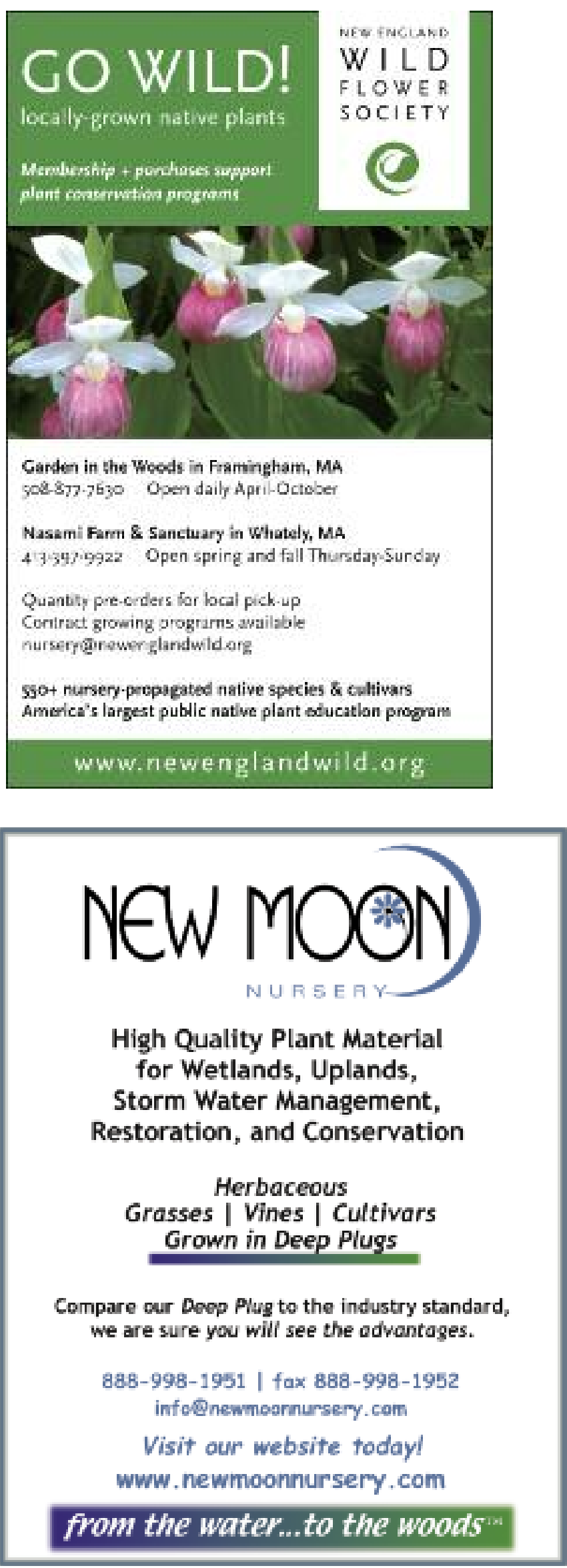
North American Prairies

Company

11988 Jarvis Ave NW,

Annadale MN 55302

Contact Tony Rieger-Borer

Ph 320.274.2906 Fax 320.274.3987

E-mail riegerborer@gmail.com

Private, wholesale, retail, mail. Plants, Seeds.

North Carolina Division of Forest

Resources-Claridge Nursery

762 Claridge Nursery Rd,

Goldsboro NC 27530

Contact James West

Ph 919.731.7988 Fax 919.731.7993

E-mail james.west@ncdenr.gov

State, retail. Plants.

North Carolina Division of Forest

Resources-Linville River Nursery

6321 Linville Falls Hwy,

Newland NC 28657

Contact Anna

Ph 828.733.5236 Fax 828.733.9399

E-mail Irnursery@skybest.com

State, wholesale, retail. Plants.

North Central Reforestation Inc 10466 405th Ave, Evansville MN 56326 Contact Dave or Michelle Olsen

Ph 218.747.2622; 877.702.5579

Fax 218.747.2621

E-mailinfo@ncrtrees.com

Private, wholesale, retail, mail, contract, M-F $8-5$, since 1978. Plants: 4,000,000/y, 95\% native, $100 \%$ propagated; container, bareroot.

See www.ncrtrees.com for inventory and information, growers of containerized tree seedlings for the Upper Midwest.

NORTH COAST NATIVE NURSERY

PO Box 660, 2710 Chileno Valley Rd,

Petaluma CA 94953

Contact David or Roanne Kaplow

Ph 707.769.1213 Fax 707.769.1230

E-mail nursery@northcoastnative

nursery.com

URL http://www.northcoastnative

nursery.com

Private, wholesale, retail, M-F 8-4, since

1987. Plants: $100 \%$ native, $95 \%$ propagated.

Propagator of quality plants native to the coastal range of northern California. Grasses, ground covers, trees, shrubs, wetland species, and perennials for habitat restoration and natural landscaping.
North Creek Nurseries Inc

388 N Creek Rd, Landenberg PA 19350

Contact Kevin Staso

Ph 610.255.0100 Fax 610.255.4762

E-mail kevins@northcreeknurseries.com

or info@northcreeknurseries.com

Private, wholesale, M-F 8-5, since 1988.

Plants: $6,000,000 / y, 70 \%$ native, $100 \%$

propagated; cuttings.

We grow a wide variety of eastern US natives and cultivars of natives.

North Dakota State Forest

Service-Towner State Nursery

878 Nursery Rd,

Towner ND 58788-9500

Contact Jeff Smette

Ph 701.537.5636 Fax 701.537.5680

E-mail tnursery@srt.com

State, wholesale. Plants.

North Sun Nurseries Inc

PO Box 299, Hwy 66 to Watabeag Rd,

Swastika ON P0K 1 T0

Contact Don Lauzon

Ph 705.567.3372 Fax 705.567.3375

E-mail northsun@ntl.sympatico.ca

Private, contract. Plants.

North Woods Nursery Inc

PO Box 149, Elk River ID 83827-0149

Ph 208.826.3408 Fax 208.826.3421

Private, wholesale. Plants.

Northeast Delta RC\&D Hardwood

Seedling Nursery

1056 Hwy 852, Rayville LA 71269

Contact Mike Adcock

Ph 318.728.7328 Fax 318.728.7326

Private, wholesale. Plants.

Northeast Natives \& Perennials

1716 E Sawmill Rd,

Quakertown PA 18951

Contact Carol Ph 215.901.5552

E-mail nenatives_perennials@

yahoo.com

Private, retail. Plants.

Northern Lights Silviculture

41481 Co Rd 63, Cohasset MN 55721

Ph 218.328.4056

E-mail gdlegler@2z.net

Plants.
Northern Pines Nursery

2300 S Morey Rd, Lake City MI 49651

Contact Diane Scholten

Ph 231.839.3277 Fax 231.839.3331

E-mail npnscholten@hotmail.com

Private, wholesale, retail. Seeds.

Northwest Native Nursery

21625 Entsminger Rd,

Arlington WA 98223

Ph 360.435.2479

Wholesale, retail. Plants.

Northwest Native Plants Inc

23501 S Beatie Rd,

Oregon City OR 97045

Contact Mike

Ph 503.632.7079 Fax 503.632.7087

E-mail nwnatives@bctonline.com

Private, wholesale, retail. Plants.

Northwest Native Seed

17595 Vierra Canyon Rd \#172,

Prunedale CA 93907

Contact Ron Ratko

Private, mail. Plants, Seeds.

Nothing but Northwest Natives

306 Wyman Rd, Woodland WA 98674

Contact Kali

Ph 360.225.6440 Fax 360.225.6440

E-mail nwplants@teleport.com

Private, wholesale, retail. Plants.

Nova Scotia Dept of Natural

Resources-Strathlone Forest

Nursery

PO Box 489, 15039 Rt 19,

Inverness NS BOE 1 NO

Contact Doug Beaton

Ph 902.258.2626 Fax 902.258.2330

E-mail beaton@bs.gov.na.ca

State, wholesale. Seeds.

NPK Nursery

3431 Oakland St, Wichita KS 67218

Contact Nathaniel Barton

Ph 316.683.0046

E-mail bar.g@cox.net

Private, wholesale. Plants. 


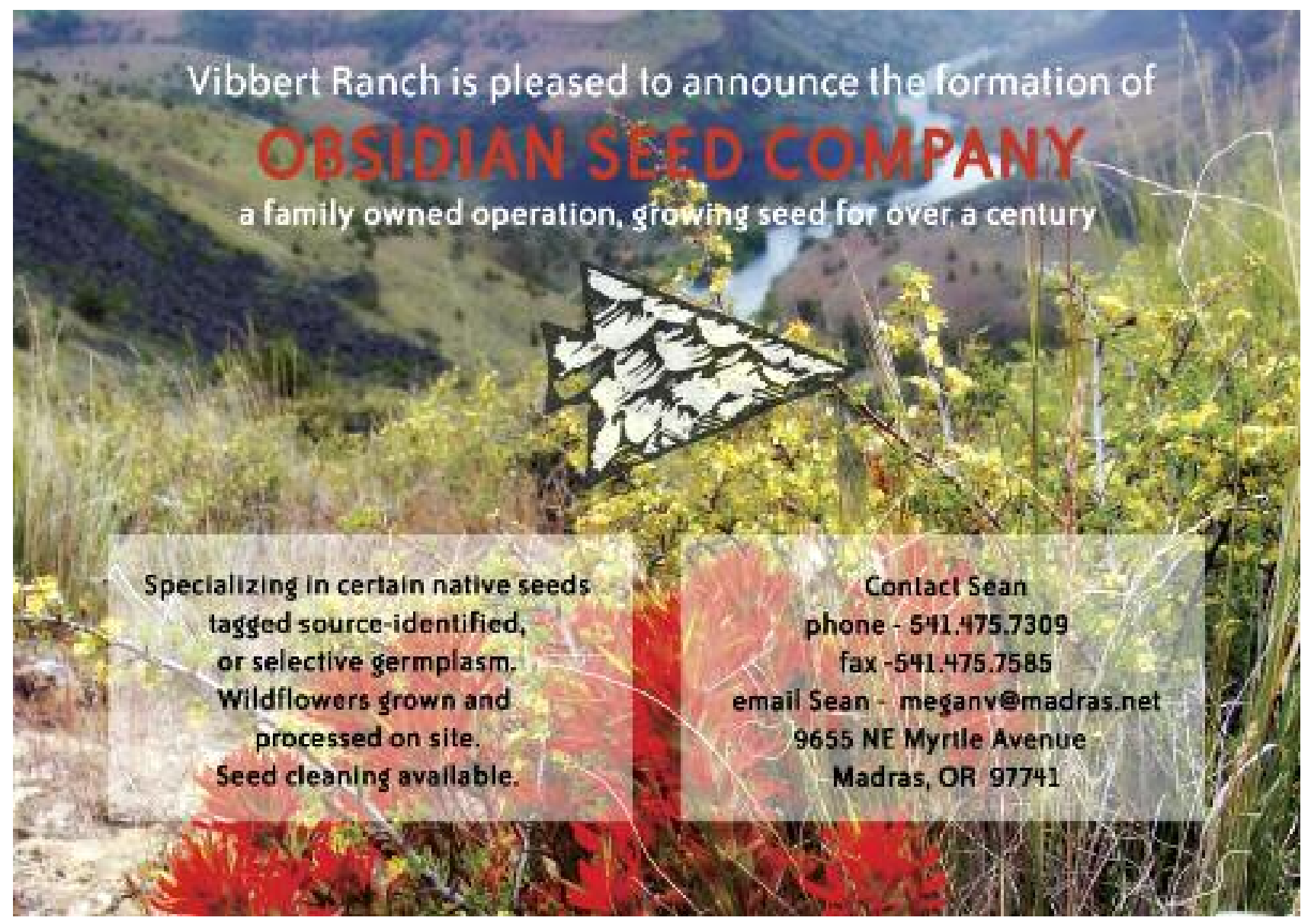

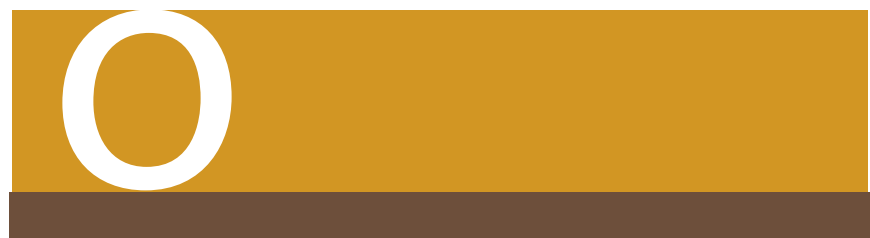

Oak Point Nursery

2300 Independence Way, Independence OR 97351 Ph 503.508.9555 E-mail sales@oakpointnursery.com Private, wholesale, retail. Plants.

Oak Prairie Farm

W 4642 Hwy 33, Pardeeville WI 53954

Contact Jim Ph 800.894.3884 Fax 800.894.3884

E-mail customercare@oakprairiefarm.com

Private, wholesale, retail. Plants, Seeds.

Oakhill Farms Native Plant

4314 Goodrich Hwy, Oakland OR 97462

Ph 541.459.2821 Fax 541.459.1361

Private, retail. Plants.

Oaks of the Wild West

10520 S Samuel Rd, Hereford AZ 85615

Contact Gary Foss Ph 520.220.0951

Private, wholesale, retail. Plants.
OASIS Environmental Native Nursery

PO Box 582, 1 9th St Island Dr, Livingston MT 59047

Contact Kallie Undem Ph 406.222.7600 Fax 406.222.7677

E-mail k.undem@oasisenviro.com or nursery@oasisenviro.com Private, wholesale. Plants.

Custom growing, wetland consulting, revegetation planting.

Oasis Tree Farms

5151 Mitchelldale B 2, Houston TX 77092

372 S Barfield Hwy, Pahokee FL 33476

Contact Andre Lacroix Ph 561.924.2400 Fax 561.924.3034

Private, wholesale. Plants.

OBSIDIAN SEED COMPANY

9655 NE Myrtle Ave, Madras OR 97741

Contact Sean Ph 541.475.7309 Fax 541.475.7585

E-mail meganv@madras.com

Private, wholesale, retail, since 1972 . Seeds: $100 \%$ native.

Specializing in certain native seeds tagged source-identified, or selective germplasm. Wildflowers grown and processed on-site. Seed cleaning available.

See ad on page 201. 
OCTORARO NATIVE

PLANT NURSERY

6126 Street Rd, Kirkwood PA 17536

Contact Jim MacKenzie

Ph 717.529.3160 Fax 717.529.4099

E-mail octoraro@octoraro.com

URL http://www.octoraro.com

Private, wholesale, Internet, contract,

8-3:30, since 1990 . Plants: $250,000 / y, 99 \%$

native, $75 \%$ propagated, $75 \%$ wild collect-

ed; container, cuttings, bioengineering; tree,

shrub, wetland/aquatic.

Octoraro Native Plant Nursery is a wholesale nursery specializing in quality container-grown, mid-Atlantic and Northeastern native trees and shrubs. Sizes range from container-grown seedlings to \#7 containers.

See ad on page 203.

O'Donnells Fairfax Nursery

1700 Sir Francis Drake Blvd,

Fairfax CA 94930

Contact Rebecca

Ph 415.453.0372 Fax 415.453.3109

Private, retail. Plants, Seeds.

OHIO DIVISION OF FORESTRY -

MARIETTA STATE NURSERY

PO Box 428, 27515 St Rt 7,

Reno OH 45750

Contact Roger D Hendershot

Ph 740.373.7410

E-mail roger.hendershot@dnr.state.oh.us

URL http://www.ohiodnr.com/forestry/

seedling

State, wholesale, retail, 7-3:30, since 1925.

Plants, Seeds. Min: 100 seedlings.

Ohio Conservation Seedlings include native species that are protected with science-based quality attributes of seed source selection and target seedling form.

Ohio Prairie Nursery

PO Box 174, Hiram OH 44234

Contact Bob Kehres

Ph 866.569.3380 Fax 330.569.7090

E-mail info@ohioprairienursery.com

Private, retail, mail, M-F 9-4:30, since 1997. Seeds: $100 \%$ native.

Oikos Tree Crops

PO Box 19425, Kalamazoo MI 49019

Contact Ken Asmus

Ph 269.624.6233 Fax 269.624.4019

Private, wholesale. Plants.
Oklahoma Dept of Agriculture-

Albert Engstrom Forest

Regeneration Center

830 NE 12th Ave, Goldsby OK 73093

Contact Scott Huff

Ph 405.288.2385 Fax 405.288.6326

E-mail scott.huff@oda.state.ok.us

State, wholesale. Seeds.

Old Ridge Nursery

PO Box 334, Calais ME 04619-0334

Contact David McLaughlan

Ph 506.466.2770 Fax 506.465.0881

E-mail oldridge@nbnet.nb.ca

Private, wholesale, retail, mail. Plants.

Old South Nurseries

693 McNatt Rd, Spruce Pine AL 35585

Contact Toby Hutcheson

Ph 256.627.4704

Wholesale, mail. Plants.

Ole Henry Gardens

665 W Broadway, Muskegon MI 49441

Ph 231.733.1876

Private, wholesale, retail. Plants.

Olympic National Park

Native Plant Nursery

600 E Park Ave,

Port Angeles WA 98362-6798

Contact Matt Albright

Ph 360.565.3130 Fax 360.565.3147

Federal. Plants.

Olympic Nursery

16215 140th Place NE,

Woodinville WA 98072

Contact Thomas Quigley

Ph 425.483.9254 Fax 425.485.9451

E-mail sales@olympicnursery.com

Private, wholesale, retail. Plants.

Oma's Garten Pflanzen

2301 Old FM 440 Rd, Killeen TX 76549

Contact Walter Daude

Ph 254.526.8792

E-mail omas.garten@hot.rr.com

Private, retail. Plants.

One Garden I

Elixir Farm Botanical

Co Rd 158, Brixey MO 65618

Ph 417.679.1003 Fax 417.261.2355

E-mailshanti@one.garden.org

Retail. Plants, Seeds.
O'Neal Nursery

4420 Harrison Ferry Rd,

McMinnville TN 37110

Contact Dwight O'Neal

Ph 931.668.7749 Fax 931.668.7855

E-mail onealnursery@blomand.net

Private, wholesale, retail. Plants.

Ontario Native Land

5258 Old Brock Rd,

Claremont ON L1Y 1A1

Contact Charles Kinsley

Ph 416.889 .4873

E-mail nativeland@nativeplants.ca

Plants.

Oregon Native Plant Nursery

PO Box 886, Woodburn OR 97071-0886

Contact Douglas M Chadwick

Ph 503.981.2353

E-mail oregonnativeplant@yahoo.com

Retail, mail, contract. Plants.

OREGON WHOLESALE SEED

PO Box 885, 5648 Evans Valley Loop,

Silverton OR 97381

Contact Angela Rose

Ph 503.874.8221 Fax 503.873.8861

E-mail flowerseed@verizon.net

URL http://www.oregonwholesaleseed.com

Private, wholesale, M-F 8-5, since 2001.

Seeds: herbaceous, grass.

Many top quality native wildflowers and grasses for sale as well as contract production, seed processing, and seed cleaning. More than 5 generations of experience with seeds!

See ad on page 203.

Oregon-Idaho Native Plant Seed

Growers Association

5000 NW 1st Ave,

New Plymouth ID 83655

Contact Mr Skinner Ph 208.278.3789

E-mail jskinner261@msn.com

Seeds.

Ornamental Plants and Trees Inc

1171 Co Rd 20 A, Hawthorne FL 32640

Contact David Dickerson

Ph 352.481.0038 Fax 352.481.3366

Private, wholesale. Plants.

Osage Prairie Mercantile

PO Box 152, Clinton MO 64735

Ph 660.885.6127

E-mailinfo@prairiesource.com

Wholesale, retail, contract. Plants. 
Osenbach Grass Seed

11009 542nd St, Lucas IA 50151

Contact John Osenbach

Ph 800.582.2788; 641.766.6792 Fax 641.766.6795

E-mail johno@iowatelecom.net

Private, wholesale, retail. Seeds.

Our Bamboo Nursery

30 Myers Rd, Summertown TN 38483-7323

Contact Adam or Sue Turtle

Ph 931.964.4151 Fax 931.964.4228

Private, wholesale. Plants.

Outback Nursery Inc

15280 110th St S, Hastings MN 55033

Contact Tom Tennant

Ph 651.438.2771 x17 Fax 651.438.3816

E-mail tom@outbacknursery.com or info@outbacknursery.com Private, wholesale, retail, M-F 8-4:30, since 1994. Plants: 250,000/y, $100 \%$ native, $100 \%$ propagated; container, cuttings.

Provider of native trees, shrubs, wildflowers, and grasses. Container grown.

Ozark Wildflower Company

HC 70 Box 169, Jasper AR 72641

Contact Ron Rottman Ph 870.446.5629

E-mail dogwood@eritter.net

Private, wholesale, retail, by appt, since 2001 . Plants: $40 \%$ native, $100 \%$ propagated.

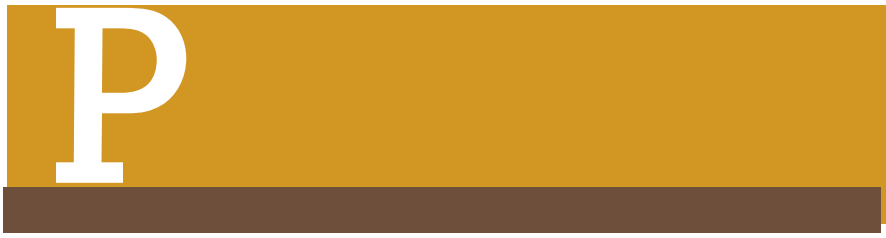

Pacific Coast Seed

533 Hawthorne Pl, Livermore CA 94550

Contact David Gilpen

Ph 925.373.4417 Fax 925.373.6855 E-mail info@pcseed.com Private, wholesale. Seeds.

\section{Pacific Natives and Ornamentals}

PO Box 23, Bothell WA 98041

Contact Rob Ph 425.483.8108 Fax 425.487.6198

E-mail pacificnatives@verizon.net

Private, wholesale. Plants.

\section{Pacific Northwest Natives}

1525 Laurel Heights Dr NW, Albany OR 97321

Contact Craig Edminster

Ph 541.928.8239 Fax 541.924.8855

E-mail pacificnwnatives@comcast.net

Private, wholesale, retail, mail, contract. Plants.
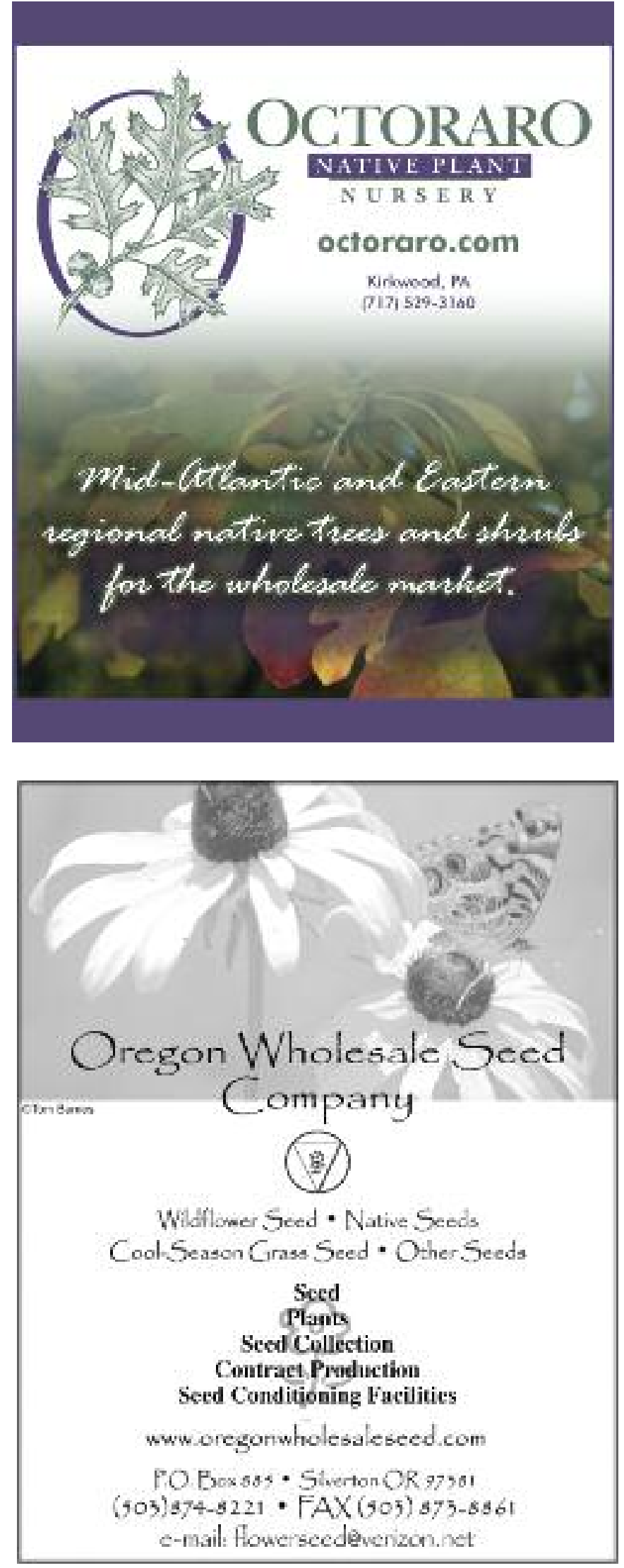

See ad on page 205. 
Pacific Rim Native Plants Ltd PO Box 413, Chilliwack BC V2P 6J7 Contact Paige or Pat Woodward Ph 604.792.9279 Fax 604.792.1891

E-mail paige@hillkeeps.ca

Private, wholesale, retail. Plants, Seeds.

Pagosa Nursery Company

1083 Deer Trail, 166 Bastille PI,

Pagosa Springs CO 81147

Contact Nancy Hanes

Ph 970.731.4126 Fax 970.731.5195

E-mail pagosafeedandseed@live.net

Private, retail. Plants.

Pajarito Greenhouse

238 Rio Bravo, Los Alamos NM 87544

Contact Dave Fox

Ph 505.672.3023

Private, retail. Plants.

Palisade Greenhouse

3895 N River Rd, Palisade CO 81526

Contact Nikki Schmalz;

Tom or Carolyn McKee

Ph 970.464.5133; 800.876.0254

Fax 970.464 .0842

E-mail

carolyn@palisadegreenhouse.com

Private, wholesale, mail. Plants.

Palouse-Clearwater

Environmental Institute (PCEI)

Learning Nursery

PO Box 8596, 1040 Rodeo Dr,

Moscow ID 83843

Contact Tracy Brown

Ph 208.882.1444 Fax 208.882.8209

E-mail brown@pcei.org

Nonprofit, retail. Plants.

PAN'S ACRES NURSERY

PO Box 113, 136 Colburn Rd,

Canterbury CT 06331

Contact Denise Ciastko

Ph 860.662.0203; 860.546.9376

Fax 860.546.6875

E-mail dciastko@ctol.net or

dee.grower@pansllc.com

URL http://www.natinc.org

Private, wholesale, retail, by appt, since

1994. Plants: $94 \%$ native, $100 \%$ propagated.

Our continuing mission is the propagation/growing of native plants harvested from seed/cuttings from local areas to preserve New England's natural heritage.
Pat Ford's Nursery Inc

8400 96th Court S,

Boynton Beach FL 33437

Contact Pat Ford

Ph 561.732.3653 Fax 561.732.3653

E-mail pat@patfordsnursery.com

Private, wholesale. Plants, Seeds.

Paul's Seeds

PO Box 355, 92 Church St,

Eureka UT 84628

Contact Paul

Ph 435.433.6924

E-mail xeriseeds@yahoo.com

Private, retail. Seeds.

Pawnee Buttes Seed Inc

PO Box 100, 605 25th St,

Greeley CO 80632

Contact Don Hijar or David Moore

Ph 800.782.5947 Fax 970.356.7263

E-mailinfo@PawneeButtesSeed.com

Private, wholesale, retail. Seeds.

PE Allen Ranch Supply

89400 497th Ave, Bristow NE 68719

Contact Paul Allen

Ph 402.583.9924 Fax 402.583.9924

E-mail paulallen@nntc.net

Private, wholesale, retail. Seeds.

Peach Springs Nursery

1155 N FM 2869

Winnsboro TX 75494

Contact Wayne Barrick

Ph 903.629.3262 Fax 903.629.3001

E-mail wayne@peachspringsnursery.com

Private, wholesale. Plants.

Pearson's Tree Place

PO Box 1175, Canutillo TX 79835

Ph 915.877.3808 Fax 915.877.3808

Wholesale, retail. Plants.

Pechanga Band of Luiseno

Indians

PO Box 1477, Temecula CA 92593

Contact Willy Pink

Ph 909.695.2768 Fax 909.695.1778

E-mail tthompson@pechanga.org

Tribal, wholesale. Plants.
PEEL'S NURSERIES LTD

11610 Sylvester Rd,

Mission BC V2V 4J1

Contact Bruce or Lauren Peel

Ph 604.820.7381 Fax 604.820.7382

E-mail peels@uniserve.com

URL http://www.peelsnurseries.com

Private, wholesale, 8-4:30, since 1991.

Plants: $100 \%$ native, $100 \%$ propagated.

Propagating and growing native plant material ranging from rooted cuttings and seedlings to container and $B \& B$ for landscape, restoration,

rehabilitation, mitigation; contract growing.

Pelton Reforestation Ltd

12930 203rd St,

Maple Ridge BC V2X 4N2

Contact David Lloyd or Jay Pelton

Ph 877.PEL.TONS Fax 604.465.7719

E-mailinfo@pelton.com

Plants.

Pelton's Nursery Inc

PO Box 560912, Miami FL 33256-0912

Ph 305.447.7667 Fax 305.233.7188

E-mail PeltonllI@aol.com

Private, wholesale. Plants.

Pennsylvania Bureau of

Forestry-Penn Nursery

137 Penn Nursery Rd,

Spring Mills PA 16875

Contact Tina M Alban

Ph 814.364.5150

E-mail penn@state.pa.us

State, 8-4, since 1922. Plants: $80 \%$ native, $100 \%$ propagated.

Pennsylvania State Game

Commission-Howard Nursery

197 Nursery Rd, Howard PA 16841

Contact Clifford Guindon

Ph 814.355.4434 Fax 814.355.8094

E-mail cguindon@state.pa.us

State, wholesale, retail, mail, Internet. Plants.

Perennial Gardens

13139224 St,

Maple Ridge BC V4R 2P6

Contact Elke Knechtel

Ph 604.467.4218 Fax 604.467.3181

E-mailinfo@perennialgardener.com

Private, wholesale. Plants. 
Performance Nursery

2501 Manhattan Beach Blvd, Redondo Beach CA 90266

Ph 310.297.6866 Fax 310.536.9173

Wholesale, retail. Plants.

\section{PERKINS NURSERY INC}

PO Box 2460, LaBelle FL 33975

2575 Case Rd, LaBelle FL 33935

Contact Dan or Debbie Perkins

Ph 863.675.3006 Fax 863.675.8281

E-mail spalmetto@aol.com

URL http://www.perkinsnursery.com

Private, wholesale, retail, contract, M-Sat 8-4:30, since 1977.

Plants: $700,000 / y, 100 \%$ native; tree, shrub. Seeds: $100,000 \mathrm{lb} / \mathrm{y}$;

tree, shrub. Min: $1 \mathrm{lb}$.

Private, wholesale, retail, plants, seed supply since 1977. World's largest supplier of saw palmetto, quart to 100 gallons. We also grow needle palm, sabal minor, and coontie. All are native to the southeastern US.

Peter Schramm—Prairie Restorations

766 Bateman St, Galesburg IL 61401

Contact Peter

Ph 309.343.2608 E-mail pschram@galesburg.net

Private, retail. Seeds.

Peterson's Riverview Nursery LLC

873 26th St, Allegan MI 49010

Contact Jerry Peterson

Ph 269.673.2440 Fax 269.673.6250

E-mail jptrees@accn.org

Private, wholesale. Seeds.

Phelan Garden I DBA Phelan Corporation 4955 Austin Bluffs Parkway, Colorado Springs CO 80918 Contact Mark Phelan Ph 719.574.8058

Private, retail. Plants.

Phillips Nurseries Inc

PO Box 355, Montchanin DE 19710

Contact Ron Ph 610.459.1127

Private, retail. Plants.

\section{PIERSON NURSERIES INC}

291 Waterhouse Rd, 24 Buzzell Rd, Dayton ME 04005

Contact Dale Pierson

Ph 207.499.2994 Fax 207.499.2912

E-mail dpierson@piersonnurseries.com

URL http://www.piersonnurseries.com

Private, wholesale, M-F 7-5, since 1976. Plants: 35\% native,

$100 \%$ propagated; container, bareroot, $B \& B$, bioengineering; tree, shrub, herbaceous, wetland/aquatic. Seeds: $100 \%$ native, $25 \%$ propagated, $5 \%$ wild collected.

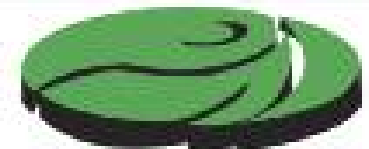

Pacific Northwest Natives

Contract producer and distributor of natlve grasses herbaceous forbs, legumes and wildflowers adapted to Western Oregon, Washington, Alaska, Northern California \& British Columbia.

\section{Source ID native seed for emeropent} wetlands, riparian stream banks, wet prairies and upland savanna.
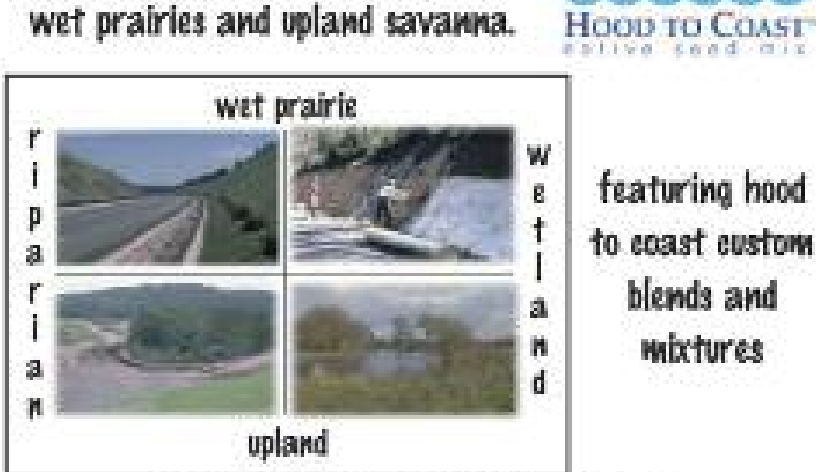

featuring hood

to eoast custom

blends and mixtures

1525 Laurel Heights Or. NW - Albany OR 97321

$541.928 .8239+$ fax $541.924 .8855+$ cell 503.580 .6455

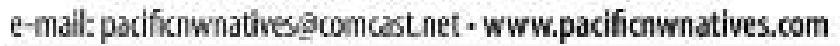

\section{directory key}

\section{NATIVE PLANTS-R-US}

123 Main Street, Anytown ID 83843

Contact John Q Public

Ph 800.555.1212 Fax 800.555.1212

BUSINESS
TYPE -mail plants-r-us@somewhere.com

$\begin{aligned} & \text { YEARS IN } \\ & \text { BUSINESS } \\ & \begin{array}{c}\text { TYPES OF } \\ \text { PLANTS }\end{array}\end{aligned} \mid \begin{aligned} & \text { URL http://www.nativeplantnetwork.com } / \text { BUSINESS } \\ & \text { POURS }\end{aligned}$

$\%$ of trees, shrubs, forbs, 350,000/y, 200 tons/y. AMOuNT

ANNUAL
PRO- $100 \%$ native, $85 \%$ propagated, $15 \%$ wild collected. OF PLANTS

PRO-
DUCTION

THAT Min wholesale $\$ 500$, retail none.

IS NATIVE

Services: on-line catalog and ordering. \% OF ANNUAL PRODUCTION THAT IS ORDER

OTHER

SERVICES
OFFERED

We offer more than 600 species of plants native to collected, source-identified Palouse Prairie plants. Our staff can help you plan your restoration project, facilitate collection and amplification of local seed sources, and supervise the restoration.

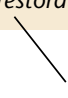

ADDITIONAL INFORMATION 
Pikes Peak Nurseries

8289 Rt 422 Hwy E,

Penn Run PA 15765

Contact David

Ph 800.787.6730 Fax 724.463.0775

E-mail pikespak@sgi.net

Private, wholesale. Plants.

Pine Breeze Nursery

PO Box 702, 7697 Beach Daisy Ln,

Bokeelia FL 33922-0702

Contact Harold

Ph 239.283.7200 Fax 941.283.7200

Private, wholesale. Plants.

Pine Grove Nursery Inc 152 Pine Grove Nursery Rd, Clearfield PA 16830-9154

Ph 814.765.2363 Fax 814.765.2363

E-mail rschickling@pinegrovenursery.com Private, wholesale. Seeds.

Pine Ridge Gardens

PO Box 200, 832 Sycamore Rd,

London AR 72847

Contact MaryAnn King

Ph 479.293.4359 Fax 479.293.4659

E-mail office@pineridgegardens.com

Private, retail, by appt, since 1992 . Plants:

$90 \%$ native, $80 \%$ propagated.

Pinelands Nursery Inc

323 Island Rd, Columbus NJ 08022

Contact Fran Chismar

Ph 609.291.9486; 800.667.2729

Fax 609.298.8939

E-mail sales@pinelandsnursery.com

Private, wholesale, contract. Plants.

Pinelands Nursery Inc

324 Co Hwy 112,

Gloversville NY 12078

Contact Fran Chismar

Ph 609.291.9486 Fax 609.298.8939

E-mail sales@pinelandsnursery.com

Private, wholesale. Plants, Seeds.

Pinelands Nursery Inc

8877 Richmond Rd, Toano VA 23168

Contact Fran Chismar

Ph 609.291.9486 Fax 609.298.8939

E-mail sales@pinelandsnursery.com

Private, wholesale, retail. Plants, Seeds.
PINEVIEW HORTICULTURAL

\section{SERVICES}

PO Box 757, 10188 N Taryne St, Hayden ID 83835

Contact lack Zimmer, PhD

Ph 208.772.7294 Fax 208.772.7598

E-mail seedguys@verizon.net

Private, wholesale, retail, M-F 7:30-5, since

2004. Seeds: $15 \%$ native; tree, shrub, herba-

ceous, grass.

Wholesale sales of seed, fertilizers, erosion control products, and professional pesticides.

Consulting on wetland mitigation, conservation reserve program plantings, erosion control, establishment and maintenance of landscapes and pasture lands, as well as storm management planning. Conservation Reserve program plantings, landscape plantings, and pasture management.

Plant Creations Inc

28301 SW 172 Ave,

Homestead FL 33030

Contact Ken Cook

Ph 305.248.8147 Fax 305.248.8151

E-mail plcrnu@comcast.net

Private, wholesale. Plants.

Plant Delights Nursery

9241 Sauls Rd, Raleigh NC 27603

Contact Tony Avent

Ph 919.772.4794 Fax 919.662.0370

E-mail office@plantdelights.com

Private, retail, mail, Internet, Phone orders/Customer Service M-F 8-5; Retail sales via appointment \& open house weekends, since 1988. Plants: $100 \%$ propagated; container.

Rare and unusual perennials/natives, including adiantums, agaves, amorphophallus, arisaemas, calanthes, clematis, colocasias, crinums, epimediums, hedychiums, hellebores, hostas, paeonias, polygonatums, rohdeas, salvias, tricyrtis, and zephyranthes.

Plant Oregon

8677 Wagner Creek Rd,

Talent OR 97540

Contact Daniel Bish

Ph 541.535.3531 Fax 541.535.2537

E-mail dan@plantoregon.com

Private, wholesale, retail. Plants.

See ad on page 207.
Plant Propagation

Technologies Inc

PO Box 599, 250 S Crawford,

Las Cruces NM 88005

Contact Rick Eaknes

Ph 505.527.9820 Fax 505.541.8821

E-mail webmaster@plantpropagation.com

Private, wholesale. Plants.

Plantas nativa LLC

PO Box 5271,

Bellingham WA 98227-5271

Contact Bay Renaud

Ph 360.715.9655 Fax 360.734.6612

E-mail bay@plantasnativa.com

Private, wholesale. Plants, Seeds.

PLANTAS NATIVA LLC EAST

303 Twisp River Rd, Twisp WA 98856

Contact Camden Shaw

Ph 509.341.4133; 509.997.0273

E-mail camden@pneast.com

URL http://www.pneast.com

Private, wholesale, retail, M-F 8-4 by appt, since 1997. Plants: $100 \%$ native. Seeds: $100 \%$ native.

Specializing in ecological restoration. Native plant restoration and landscaping contractor Non-systemic pasture management. Project design, installation, and maintenance since 1997.

See ad on page 207.

Plantasia Cactus Gardens

867 Filer Ave W, Twin Falls ID 83301

Contact LaMar Orton

Ph 208.734.7959

E-mail lorton1@msn.com

Private, retail, mail. Plants, Seeds.

Plantation Discount Nursery 105 Lindbergh, El Paso TX 79932

Ph 915.584.0433 Fax 915.585.9585

Private, wholesale, retail. Plants.

Plantra Inc

2508 Northland Dr,

Mendota Heights MN 55120

Contact Mary Jo

$\mathrm{Ph}$ 800.951.3806; 651.686.6688

Fax 651.681.2067

Private, wholesale, retail. Plants. 
Plants for Dry Places

25735 Garboni Rd,

Menifee Valley CA 92584

Ph 909.679.6612 Fax 909.670.6612

E-mail watrlady@cosmoaccess.net

Wholesale, retail, mail, contract. Plants.

Plants for the Southwest I

Living Stones Nursery

2936 N Stone Ave, 50 E Blacklidge, Tucson AZ 85705

Contact Gene Joseph or Jane Evans

Ph 520.628.8773 Fax 520.628.8773

Private, wholesale, retail. Plants.

Plants of the Southwest

3095 Agua Fria Rd, Santa Fe NM 87507

Contact Betty Campbell

Ph 800.788.7333

E-mail plantsofthesouthwest@hotmail.com

Private, wholesale, retail, M-Sat 8-5; Sun during summer, since 1973.

Plants, Seeds.

PLANTS OF THE WILD

PO Box 866, 123 State Line Rd, Tekoa WA 99033

Contact Kathy Hutton

Ph 509.284.2848 x110 Fax 509.284.6464

E-mail kathy@plantsofthewild.com

URL http://www.plantsofthewild.com

Private, wholesale, retail, mail, Internet, contract, M-F 8-4, since 1979. Plants: $1,000,000 / y, 90 \%$ native, $95 \%$ propagated, $5 \%$ wild collected; tree, shrub, herbaceous, grass, wetland/aquatic. Seeds:

herbaceous, grass.

Providing high-quality container-grown Pacific Northwest natives to our customers for 30 years. More than 100 different species of trees, shrubs, forbs, and grasses. Seed, liners, and larger containers available.

The Plantworks LLC

1805 U Ave, La Grande OR 97850

Contact Sandra Roth or Richard Kenton

Ph 541.786.5122 Fax 541.963.7870

E-mail plantworks@oregontrail.net

Private, wholesale, please call, since 1994. Plants: 100\% native, $99 \%$ propagated; container. Seeds: $99 \%$ native, $99 \%$ wild collected.

PLEASANT AVENUE NURSERY INC

PO Box 1669, 506 S Pleasant Ave,

Buena Vista CO 81211-1669

Contact Gary or Mary Ludwig

Ph 719.395.6955 Fax 719.345.5718 E-mail pan@amigo.net

URL http://www.pleasantavenuenursery.com

Private, retail. Plants.

Native trees, shrubs, and wildflowers grown in central Colorado at an elevation of $8000 \mathrm{ft}$. We've been growing since 1972. Great plants and great service!
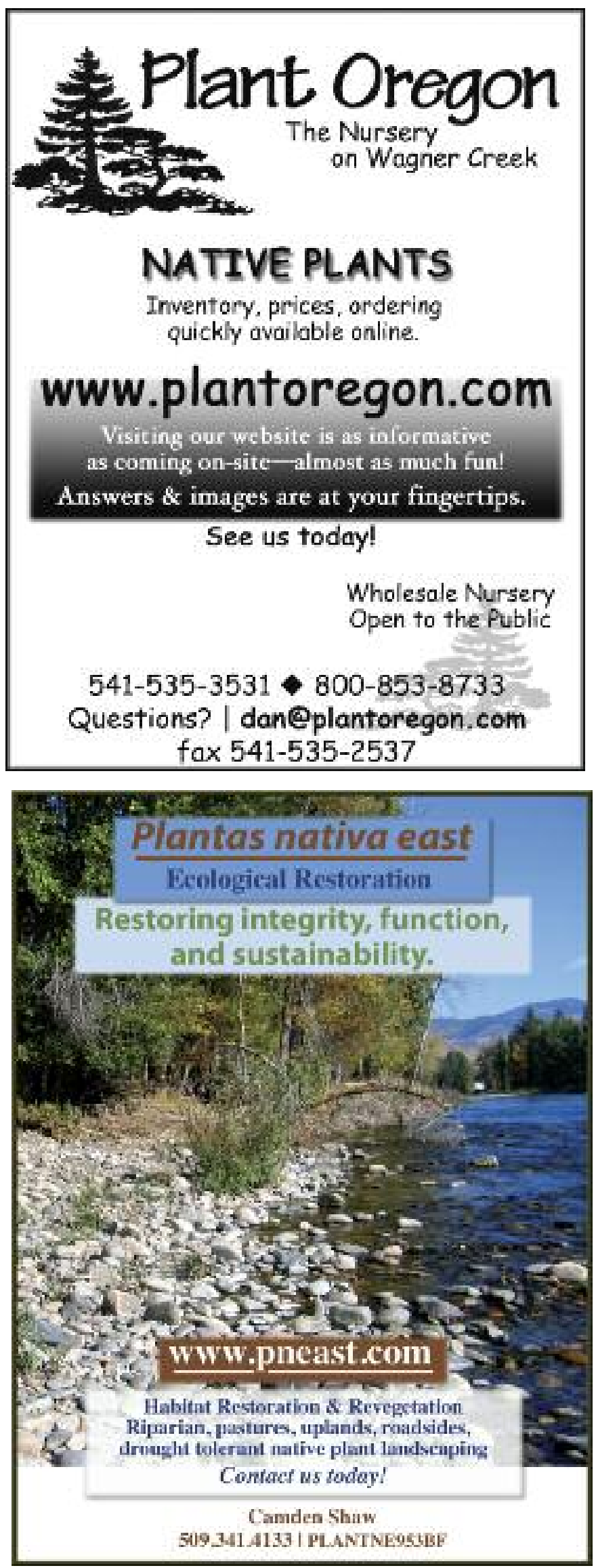
Plum Creek Timber CompanyCottage Grove Nursery

76928 Mosby Creek Rd, Cottage Grove OR 97424

Contact Steve Hutchison

Ph 541.942.5516

E-mail steven.hutchison@plumcreek.com

Private, wholesale. Plants, Seeds.

Plum Creek Timber Company-

Jesup Nursery

1689 Nursery Rd, Jesup GA 31546

Contact Ken Woody

Ph 912.427.4871; 206.467.3600

Fax 912.530 .8438

E-mail ken.woody@plumcreek.com

Private, wholesale. Plants, Seeds.

Plum Creek Timber Company-

Pablo Montana Forest Nursery

PO Box 188, 36307 Light Rd,

Pablo MT 59855

Contact Ken Cameron

Ph 406.675.3500 Fax 406.675.3504

E-mail Kcameron@plumcreek.com

Private, wholesale, retail. Plants.

Plum Creek Timber Company-

Pearl River Nursery

1032 Camp Ln, Hazelhurst MS 39083

Contact Ken McQuage

Ph 601.894.1072 x8108

E-mail ken.mcquage@plumcreek.com

Private, wholesale. Plants, Seeds.

Plum Creek Timber Company-

Shubuta Nursery

1444 Shubuta Eucutta Rd,

Shubuta MS 39360

Ph 601.687.5766

Private. Plants.

Plum Creek Timber Company-

Vivian Nursery

410 Lake Rd, Bivins TX 75555

Ph 903.672.4625 Fax 903.672.4627

Private, wholesale. Plants, Seeds.

Plumline Nursery

4151 Logan Ferry Rd,

Murrysville PA 15668

Contact Karen

Ph 724.327.6775 Fax 724.327.9210

E-mail karen@plumlinenursery.com

Private, wholesale, retail. Plants.
PLUMMER SEED COMPANY

PO Box 70, Ephraim UT 84627

Contact Mark Plummer

Ph 435.283.4844; 435.851.0715

Fax 435.283.4030

E-mailmark@plummerseedco.com

Private, wholesale, retail, M-F 8-5, since

1982. Seeds: $100 \%$ native, $90 \%$ wild collect-

ed; shrub, herbaceous.

Plummer seeds are well-suited for reclamation projects as well as for landscaping applications.

See ad on page 209.

Ponderosa Nursery

PO Box 158, 4913 133rd Ave,

Hamilton MI 49419

Contact Carrie

Ph 269.751.6031; 800.228.3021

Fax 616.751 .8829

E-mail carrie@ponderosanursery.net

Private, wholesale. Plants.

Portland Nursery

5050 SE Stark, Portland OR 97215

Ph 503.231.5050; 503.788.9000

Fax 503.231.7123

Private, retail. Plants.

Possibility Place Nursery

7548 W Manhattan Rd,

Monee IL 60449

Contact Connor Shaw

Ph 708.534.3988 Fax 708.534.6272

Private, wholesale. Plants.

Postlewait Nursery

28923 S Barlow Rd, Canby OR 97013

Ph 503.651.2393 Fax 503.651.3156

E-mailmpostlewait@canby.com

Private, wholesale, retail, contract. Plants.

Pots \& Plants Garden Center

5902 Bee Cave Rd, Austin TX 78746

Ph 512.327.4564 Fax 512.328.5965

Private, retail. Plants, Seeds.
PRAIRIE EARTH NURSERY

RR 1 Box 151, Bradford IL 61421

Contact Jim Alwill

Ph 309.897.9911

E-mail Jim_alwill@yahoo.com

Private, wholesale, retail, mail, contract, please call, since 1999 . Plants: $99 \%$ native, $100 \%$ propagated; container, bareroot; tree, shrub, herbaceous, grass. Seeds.

6-inch wide $\times 12$-inch tall contract grown-

Super Plugs-available and contract growing.

Serving all of Illinois, eastern lowa, eastern Missouri, southern Wisconsin, southern Michigan, and western Indiana. Customers include IL DOT, homeowners, public institutions, schools, volunteer groups, park districts, villages, and more. Machinery and equipment to add forbs to existing grass-dominated prairies with little or no disturbance to existing established field.

Prairie Frontier

W 281 S3606 Pheasant Run,

Waukesha WI 53189

Contact Deb Edlhuber

Ph 262.544.6708 Fax 262.544.6708

E-mail wildflower@prairiefrontier.com

Private, wholesale, retail, mail. Seeds.

Prairie Future Seed Company

PO Box 644,

Menomonee WI 53052-0644

Contact Randy Powers

Ph 262.820.0211 Fax 414.325.1228

E-mail pfsco@Wi.rr.com

Private, wholesale, retail, mail. Seeds.

Prairie Habitats Inc

PO Box 10, 0043E-82 Rd N,

Argyle MB ROC OBO

Contact John Morgan

Ph 204.467.9371 Fax 204.467.5004

E-mail jpmorgan@mts.net

Private, retail. Seeds.

Prairie Hill Farm

877 Co Rd 263, Auxvasse MO 65231

Contact Devin Chandler

Ph 573.387.4680 Fax 573.387.4875

E-mail chandler@ktis.net

Private, wholesale. Plants. 
Prairie Hill Wildflowers

8955 Lemonde Rd, Ellendale MN 56026

Contact John Hoffman Ph 507.451.7791 Fax 507.451.3812

E-mail seedman@myclearwave.net

Private, wholesale, retail. Plants, Seeds.

\section{PRAIRIE MOON NURSERY}

32115 Prairie Ln, Winona MN 55987

Contact Bill Carter

Ph 866.417.8156E-mailinfo@prairiemoon.com

Private, wholesale, retail, mail, Internet, contract, M-F 9-5, since 1982. Plants: $40,000 / y, 100 \%$ native, $100 \%$ propagated; bareroot; tree, shrub, herbaceous, grass, wetland/aquatic. Seeds: $100 \%$ native; shrub, herbaceous, grass. Min: $\$ 5 \mathrm{~S} \& \mathrm{H}$.

More than 600 species native to upper Midwest and Northeast.

Prairie Nursery

PO Box 306, W 5875 Dyke Ave, Westfield WI 53964

Contact Ann Schmitt

Ph 800.476.9453; 608.296.3679 Fax 608.296.2741

E-mail cs@prairienursery.com

Private, wholesale, retail. Plants, Seeds.

Prairie Restorations Inc

PO Box 327, 39922 128th St, Princeton MN 55371

Contact Mike Evenocheck

Ph 763.389.4342 Fax 763.389.4346

E-mailinfo@prairieresto.com

Private, wholesale, retail, mail, M-F 9-5, since 1977. Plants. Seeds:

$100 \%$ native, $100 \%$ propagated, $100 \%$ wild collected.

More than 600 species native to upper Midwest and Northeast;

http://www.prairiemoon.com.

See ad on page 211.

Prairie Seed Source

PO Box 83, North Lake WI 53064-0083

E-mail rasillon@ameritech.net or pb9@prairiebob.com

Private, retail, mail, since 1974 . Seeds: $100 \%$ native, $100 \%$ propagated.

We also do consultations for site selection, site preparation, species selection, and maintenance.

Prairie Seeds Inc

Corner of Hwy $39 \& 60$, RR 4, Calmar AB T0C 0V0

Contact Gloria Weir

Ph 780.985.7314 E-mail Gloria.weir@brettyoung.ca

Private, wholesale, retail, 8-5, since 1934. Seeds.

Prides Corner Farms

122 Waterman Rd, Lebanon CT 06279

Contact Mark Sellew Ph 860.642.7535 Fax 860.642.2027

Private, wholesale, contract. Plants.

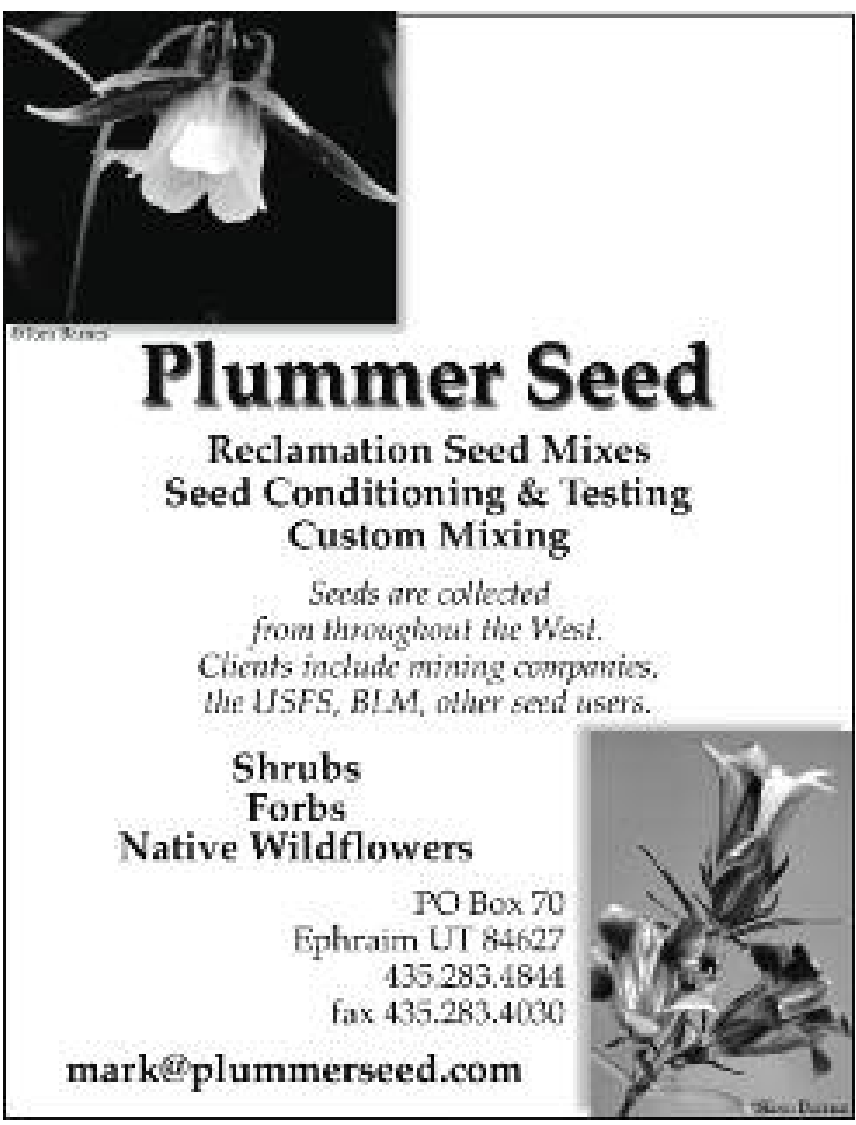

The Primrose Path

921 Scottdale-Dawson Rd, Scottdale PA 15683

Contact Oliver Ph 724.887.6756 Fax 724.887.3077

E-mail primrose@a1usa.net

Private, wholesale, retail. Plants.

Prince Edward Island Ministry of Forestry-

J Frank Gaudet Nursery

PO Box 2000, Upton Rd, Charlottetown PE C1A 7N8

Contact Bill Butler Ph 902.368.4711 Fax 902.368.4713

E-mail jfgnursery@gov.pe.ca

State. Plants.

Prindel Creek Farm Inc

95520 E Five Rivers Rd, Tidewater OR 97390

Contact Daniel Ph 541.528.3330 Fax 541.528.3330

Private, wholesale. Plants.

Progressive Plants

10252 S Hwy U 111, Copperton UT 84006

Contact Kevin Hardy or Mike

Ph 801.565.7333 Fax 801.565.7345

E-mail sales@progressiveplants.com

Private, wholesale. Plants. 
Project Native

342 N Plain Rd, Housatonic MA 01236

Contact Raina Webber

Ph 413.274.3433 Fax 413.274.3464

E-mail projectnative@gmail.com

Nonprofit, wholesale, retail. Plants, Seeds.

PRT_Oregon Nursery

31783 S Meridian Rd,

Hubbard OR 97032

Contact Mark Montville

Ph 503.651.3266

E-mail mark.montville@prtgroup.com

Private, wholesale. Plants.

PRT-Vernon Nursery

7501 Bench Rd,

Vernon BC V1H $1 \mathrm{H} 3$

Contact Chris Mostyn

Ph 250.542.4100 Fax 250.542.1200

E-mail support@prtgroup.com

Private, wholesale. Plants.

Purple Prairie Farm

Rt 2 Box 176, Wyoming IL 61491

Contact Gary

Ph 309.286.7356

E-mail garycli@theramp.net

Private, wholesale. Plants, Seeds.

Pushpetappa Gardens

2317 Washington St,

Franklinton LA 70438-2504

Contact John Thornton

Ph 985.839.4930

Private, wholesale, retail, mail. Plants.

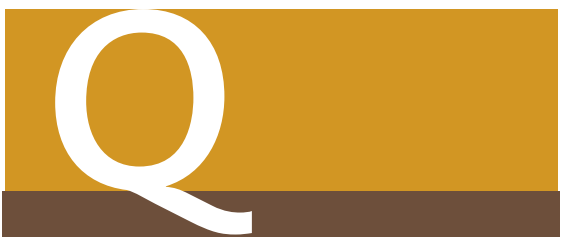

Quail Botanical Gardens

Foundation Inc

230 Quail Gardens Dr,

Encinitas CA 92024

Ph 760.436.9516 Fax 760.632.0917

E-mail info@qbgardens.com

Retail. Plants.

Quality Seed Collections

Box 1531, Kamloops BC V2C 6L8

Contact Doug or Sandy Gregory

Ph 250.374.9689 Fax 250.374.9654

210

E-mail qualityseed@telis.net

Private, wholesale. Seeds.
Quality Seeds West

PO Box 61572, Langley BC V3A 8C8

19863 38B Ave, Langley BC V3A 6G3

Contact Russ Paton

Ph 604.574.7333 Fax 604.574.7331

E-mail bill@qualityseedswest.com

Private, wholesale, retail, mail. Seeds.

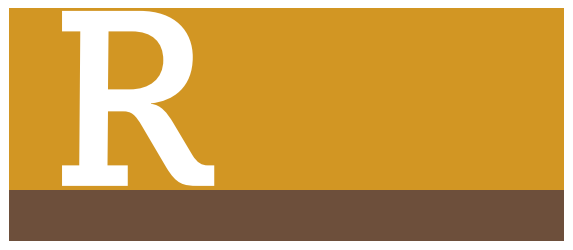

Raemelton Farm

PO Box 60663, Potomac MD 20855

2313 New Design Rd,

Adamstown MD 21710

Contact Steve Black

Ph 240.416.0714 Fax 240.990.9125

E-mail steve@raemelton.com

Private, wholesale. Plants.

Rainier Seeds Inc

PO Box 1064, 1404 Fourth St,

Davenport WA 99122

Contact Vicki Nicholls

Ph 800.828.8873; 509.725.1235

Fax 509.725.7015

E-mail ecoulter@rainierseeds.com

Private, wholesale, retail, mail. Seeds.

Raintree Nursery

391 Butts Rd, Morton WA 98356

Ph 800.391.8892 Fax 888.770.8358

E-mail customerservice@raintree

nursery.com

Private, retail, mail. Plants.

Ramah Navajo Community

Enterprise Inc-Native Plants

Nursery

PO Box 635, Ramah NM 97321

Contact Samantha Whitetail Eagle

Ph 505.879.2301

E-mail rnce@yahoo.com

Tribal, wholesale, retail. Plants.

Rancho La Orquidea Inc

1124 Pearson Rd, Milton FL 32583

Contact Alice Lezcano

Ph 850.983.8948 Fax 850.981.0144

E-mail orchidfarm@aol.com

Private, wholesale, mail. Plants.
Rancho Lomitas Native

Plant Nursery

PO Box 442, Rio Grande City TX 78582

Contact Benito Trevino

Ph 956.486.2576 Fax 956.486.2576

E-mailinfo@rancholomitas.com

Private, wholesale, retail. Plants.

Randy's Perennials \& Water

Gardens

523 W Crogan St (Hwy 29),

Lawrenceville GA 30046

Contact Randy Kucera

Ph 770.822.0676 Fax 770.822.1259

Private, retail. Plants.

Rascon Landscaping and Nursery

PO Box 69236, Tucson AZ 85737

4747 W Camino de Manana,

Tucson AZ 85742

Contact Cindy Hughes

Ph 520.297.8358 Fax 520.297.8350

Private, retail. Plants, Seeds.

Raven Nursery

22370 Indianola Rd NE,

Poulsbo WA 98370

Contact Beatrice

Ph 360.598.3323 Fax 360.598.3323

E-mail raventrees@msn.com

Private, wholesale, retail. Plants.

Rayonier Inc

PO Box 456, 11704 Baxter/Durrence

Rd, Glennville GA 30427

Contact Kelly Dougherty

Ph 912.654.4065 Fax 912.654.4071

E-mail kelly.dougherty@rayonier.com

Private, wholesale, retail. Plants.

RE Mitchell Nursery

PO Box 336, Pine Hill AL 36769

Contact MacMillan Bloedel Inc

Ph 334.682.9882 Fax 334.682.4481

E-mail rbower@mbpi.com

Wholesale, contract. Plants.

RECON NATIVE PLANTS INC

1755 Saturn Blvd, San Diego CA 92154

Contact Ryan West

Ph 619.423.2284 Fax 619.423.0632

E-mail nativeplants@recon-us.com

URL http://www.reconnativeplants.com

Private, wholesale, retail, M-F 1-4, since

1999. Plants: $950,000 / y, 100 \%$ native,

$100 \%$ propagated; container, bareroot,

$B \& B$, cuttings, bioengineering; tree, shrub,

herbaceous, grass, wetland/aquatic. Seeds: 
$100 \%$ native, $25 \%$ propagated, $75 \%$ wild collected; tree, shrub, herbaceous, grass.

We also do consultations for site selection, site preparation, species selection and maintenance.

Seeds available through contract growing. Specializing in growing hearty native plant material for habitat restoration and the landscape. Our plants are propagated from sourceidentified seeds and are 100\% native. No cultivars. Serving California, Nevada, and Arizona.

Redbud Native Plant Nursery LLC 1214 N Middletown Rd,

Glen Mills PA 19342

Contact Catherine Smith

Ph 610.358.4300 Fax 610.358.3330

E-mail catheris@mindspring.com or info@redbudnativeplantnursery.com Private, wholesale, retail, T-Sun 9-5 Mar-Nov, since 2002. Plants: $6000 / y, 100 \%$ native, $100 \%$ propagated, $0 \%$ wild collected; container.

Redbud Native Plant Nursery offers a diverse selection of native trees, shrubs, vines, perennials, grasses, and ferns.
Red's Rhodies

15920 SW Oberst Ln,

Sherwood OR 97140

Contact Red

Ph 503.625.6331 Fax 503.625.8055

E-mail red@redsrhodies.com

Private, wholesale, retail, mail. Plants.

Redwood City Seed Company

PO Box 361, Redwood City CA 94064

Contact Craig Dremann

Ph 650.325.7333 Fax 650.325.4056

Private, contract. Seeds.

Redwood Valley Rancheria

3250 Rd 1, Redwood Valley CA 95470

Contact Chuck Williams

Ph 707.485.0361 Fax 707.485.5726

E-mail redwoodres@pacific.net

Tribal. Plants.

Reed's Seeds Inc

PO Box 230, Chillicothe MO 64601

Contact EL Reed

Ph 660.646.4426 Fax 660.646.4411

E-mail reedseed@greenhills.net

Private, wholesale, retail. Seeds.
Reeseville Ridge Nursery

512 S Main St, Reeseville WI 53579

Contact Darryl J Kromm

Ph 920.927.3291 Fax 920.927.3291

E-mail rrn@charter.net

Private, wholesale, retail, mail. Plants, Seeds.

Reeves Wildflower Nursery

28431 200th St, Harper IA 52231

Contact Ray Reeves

Ph 641.635.2817

E-mail rayreeves@lisco.com

Private, wholesale, retail. Plants, Seeds.

Reflections of Nature

850688 Hwy 17 S, Yulee FL 32097

Contact Angie Loper

Ph 904.225.9915 Fax 904.225.5333

E-mail rnlnursery@comcast.net

Private, wholesale, retail. Plants.

Regional Parks Botanic Garden

Tilden Regional Park, Berkeley CA

94708-2396

Ph 510.841.8732

Retail. Plants.

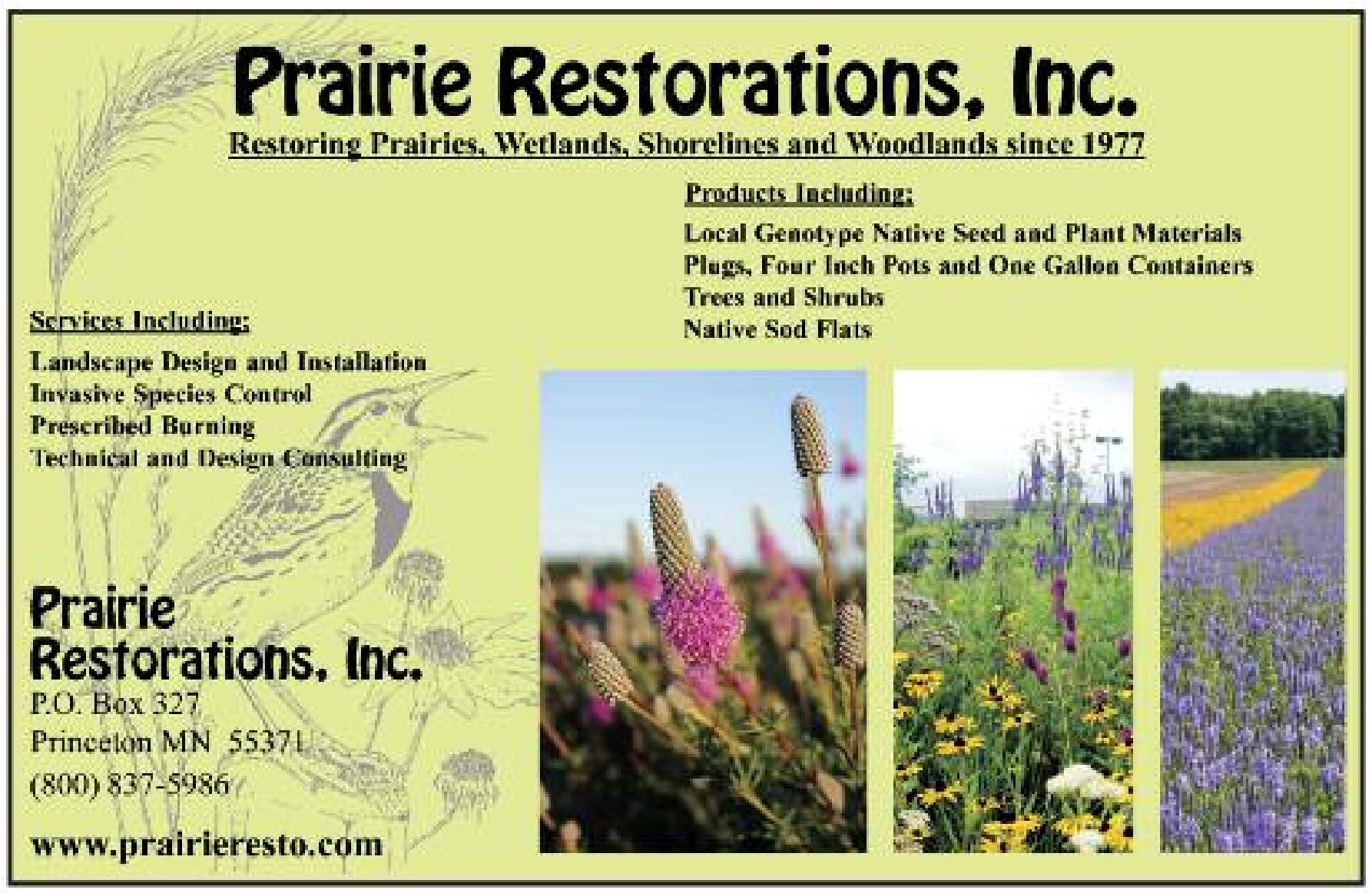


Renee's Garden

28431 200th St, Harper IA 52231

Contact Renee Shepherd

Ph 888.880.7228 Fax 831.335.7227

E-mail renee@reneesgarden.com

Private, wholesale, retail. Plants, Seeds.

Restoration Resources

3868 Cincinnati Ave, Rocklin CA 95765

Contact Kristi Burrus

Ph 916.408.2990 Fax 916.408.2999

E-mail

k.burrus@restoration.resources.net

Private, wholesale, mail. Plants.

Retzer Nature Center

S 14 W28167 Madison St,

Waukesha WI 53188

Contact Mike Bourquin

Ph 262.896.8007 Fax 262.896.6822

E-mail retzer@waukeshacounty.gov

State, retail. Plants, Seeds.

The Reveg Edge

PO Box 609, Redwood City CA 94064

Contact Craig Dremann

Ph 650.325.7333 Fax 650.325.4056

E-mail craig@ecoseeds.com

Private. Plants.

The Rhododendron Species

Foundation

PO Box 3798, 2525 S 336th St,

Federal Way WA 98063

Contact Rick Peterson

Ph 253.838.4646 Fax 253.838.4686

E-mail rsf@rhodygarden.org

Private, retail. Plants.

Richardson Agco

PO Box 206, 1500 W Vega Blvd,

Vega TX 79092

Contact Wayne Richardson

Ph 806.267.2459 Fax 806.267.2997

E-mail agco@1S.net

Private, wholesale, retail. Seeds.

Rigsby Nursery Inc

PO Box 50910,

Fort Myers FL 33994-0910

Ph 239.543.3379 Fax 235.543.7115

E-mail drigsby@attglobal.net

Wholesale. Plants.
Ripley County Farms

PO Box 614, Rt 3 Box 285,

Doniphan MO 63935

Contact Harrison Wells

Ph 573.996.3449 Fax 573.996.3449

E-mail rcf@semo.net

Private, wholesale. Plants.

Riverside Native Trees

2295 River Rd, Delaware OH 43015

Contact Ed Kapraly

Ph 740.815.3230 Fax 740.943.5068

Private, wholesale, retail. Plants.

Riverside Nursery Inc

2306 W Riverside Dr, Salem VA 24153

Ph 540.387.4020 Fax 540.387.4020

E-mail botbruce@aol.com

Private, wholesale, retail. Plants.

Robinson Rancheria

PO Box 1580, 1545 E Hwy 20,

Nice CA 95464

Contact Meyo Maruffo

Ph 707.275.0205 Fax 707.275.0470

E-mailmmarrufo@robinsonrancheria.org

Tribal, contract. Plants.

Rock Bottom Ranch Koi

and Nursery

1130 Bly Mountain Cutoff,

Bonanza OR 97523

Ph 541.545.6481 Fax 541.545.6479

E-mail annie@kfalls.net

Private, wholesale, retail, contract. Plants.

Rock Spring Farm

82 Brighton Rd NE, Atlanta GA 30309

6025 Old $411 \mathrm{~S}$,

Chapsworth GA 30705

Contact Walter Bland

Ph 404.626.8020

E-mail jwbland@rockspringfarm.com

Private, wholesale. Plants.

Rocky Mountain Native Plants

Company

3780 Silt Mesa Rd, Rifle CO 81650

Contact Randy Mandel

Ph 970.625.4769 Fax 970.625.3276

E-mail native@rmnativeplants.com

Private, wholesale. Plants.
Rocky Mountain Pines

555 Ute Hwy, Longmont CO 80503

Contact John Hulme

Ph 303.823.6658; 800.795.8663

Fax 303.823.0512

Private, wholesale. Plants.

Rocky Mountain Rare Plants

1706 Deerpath Rd,

Franktown CO 80116-9462

Contact Bob Skowron

Fax 303.265.9263

E-mail staff@rmrp.com

Private, retail, mail. Seeds.

ROHDES NURSERY

1651 Wall St, Garland TX 75041

Contact Karin Gilley or Gregory Rohde

Ph 972.864.1934 Fax 972.864.0128

E-mail grohde@greensense.net

URL http://www.beorganic.com

Private, wholesale, retail, mail, M-Sat 8-5:30

Sun $10-5$, since 1972 . Plants: $30 \%$ native;

container, bareroot.

Rohdes is the oldest ongoing organic nursery in north Texas and the first commercial property to be certified as a Wildlife Habitat by both the Texas Wildscapes and the US Parks and Forest

Federation. By combining native plants with hardy woody ornamentals, interesting landscape can be created that will enhance any property with drought-tolerant, disease- and insect-resistant plants. A little knowledge goes a long, long way.

Rolling Acres Native Landscape

Nursery LLC

2513 N Humpty Dumpty Rd,

Reedsville WI 54230

Contact James

Ph 877.205.9817

E-mail rolling.acres.native.nursery@earth link.net

Private, wholesale, retail. Plants.

ROLLING GREEN NURSERY

64 Breakfast Hill Rd,

Greenland NH 03840

Contact Beth Simpson

Ph 603.436.2732 Fax 603.436.2309

E-mailinfo@rollinggreennursery.com URL http://www.rollinggreennursery.com Private, retail, M-Sat 8-5 Sun 10-4, since 1976. Plants: $40,000 / y, 20 \%$ native, $80 \%$ propagated; container; shrub, herbaceous.

Retail growers of native and ornamental perennials, trees, and shrubs-zone 5-with emphasis on sea coast hardy plants. 
Rose Hill Nursery

2282 Teller Rd, Rose Hill IA 52586

Ph 515.632.8308

E-mail rockman49@hotmail.com

Private, retail, mail. Plants.

Roseburg Forest Products-

Lebanon Forest

Regeneration Center

34937 Tennessee Rd,

Lebanon OR 97355

Contact Mike Albrecht

Ph 541.259.2651 Fax 541.259.3661

E-mailmjalbrecht@msn.com

Forest Industry, wholesale. Plants.

Rosso Gardens LLC

PO Box 80345, 6404 Ellis Ave $S$,

Seattle WA 98108

Contact Gene Rosso

Ph 206.763.1888 Fax 206.762.2544

E-mail jerry0716@mindspring.com

Private, wholesale, retail, mail, Internet, $\mathrm{M}-\mathrm{F}$ 8-5 Sat 9-5 seasonal hours please call, since 1958. Plants: $350,000 / y, 30 \%$ native, $30 \%$ propagated, $10 \%$ wild collected; container, bareroot, $B \& B$, cuttings, bioengineering.

Quality plants, excellent service.

Round Butte Seed Growers Inc PO Box 117, 505 C St, Culver OR 97734

Contact Britt Spaulding

Ph $541.546 .5222 \times 18$

E-mail bspaulding@rbseed.com

Private, retail, $8-5$, since 1961 . Seeds: $10 \%$ native.

RSS Field Services Inc

PO Box 549, Plant City FL 33564

Contact Jay Allen

Ph 813.754.7160; 863.285.8808

Fax 813.752.3303

E-mail rssfld@verizon.net

Private, wholesale. Plants.

Rugged Country Plants

53671 W Crockett Rd, Milton-

Freewater OR 97862

Ph 541.938.3970 Fax 541.938.8182

E-mail sales@ruggedcountryplants.com

Private, wholesale, contract. Plants.
Runway Growers Inc

2891 and 2911 SW 36th St,

Ft Lauderdale FL 33312

Contact Jamie Hayes

Ph 954.584.0269 Fax 954.584.6958

E-mail runwaygrowersjamie@gmail.com

Private, wholesale, contract. Plants.

Rutland Forest Nursery

502 Owen-Medford Rd,

Lenox GA 31637

Contact Terrell Rutland

Ph 229.382.5504

Private, wholesale. Plants, Seeds.

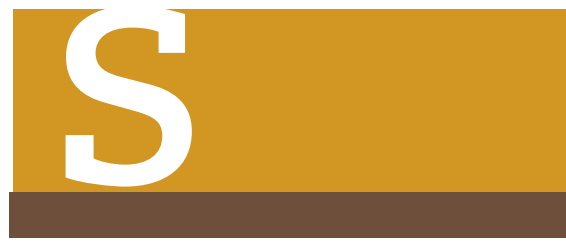

S\&S Seeds I

Albright Seed Company

PO Box 1275, 6155 Carpinteria Ave,

Carpinteria CA 93014-1275

Contact Victor Schaff

Ph 805.684.0436 Fax 805.684.2798

E-mail info@ssseeds.com

Private, wholesale. Seeds.

S\&S SEEDS I ENVIRONMENTAL

SEED PRODUCERS

PO Box 947, Albany OR 97321

Contact Mike Billman or Mike Cersovski

Ph 541.928.5868 Fax 541.928.5581

E-mailinfo@wildflowerseed.com

URL http://www.wildflowerseed.com

Private, wholesale, M-F 7:30-4:30, since

1998. Seeds: $70 \%$ native, $25 \%$ propagated,

$5 \%$ wild collected. Min: $1 \mathrm{lb}$ or $\$ 50$.

Our services include custom mix design and contract growing.

Salish \& Kootenai Tribal College

Native Plant Nursery

PO Box 117, Pablo MT 59855

Contact Dawn Thomas

Ph 406.675.4795 Fax 406.675.4800

E-mail dawn_thomas@skc.edu

Private, wholesale, retail. Plants.

San Felasco Nurseries Inc

7315 NW 126 St, Gainesville FL 32653

Contact Alan Shapiro

Ph 352.332.1220 Fax 352.332.3113

E-mail alan@grandiflora.pro

Private, wholesale. Plants.
San Marcos Growers

PO Box 6827, 125 S San Marcos Rd,

Santa Barbara CA 93105

Contact Peggy Koegler

Ph 805.683.1561 x125 Fax 805.964.1329

E-mail sales@smgrowers.com

Private, wholesale, M-F 7:30-4:30 Sat 8-12, since 1980 . Plants: $10 \%$ native, $100 \%$ propagated; container.

Sand Mountain Herbs

321 Co Rd 18, Fyffe AL 35971

Contact Larry Chandler

Ph 256.659.2726

E-mail info@sandmountainherbs.com

Private, mail, Internet. Plants, Seeds.

Sand Prairie Farms Inc

185 Rogers Rd, Ohio IL 61349

Contact Tom Yucus

Ph 815.376.6641 Fax 815.376.2321

E-mail tomyucus@yahoo.com

Private, wholesale, retail, mail. Seeds.

Sandhill Farm

1125010 Mile Rd NE,

Rockford MI 49341

Contact Cheryl S Tolley

Ph 616.691.8214 Fax 616.691.7872

E-mail cherylt@iserv.net

Private, wholesale, retail, mail. Plants.

SANDY WILSON NATIVE PLANT \& AQUATIC I SW HORTICULTURAL SERVICES

834 Church Rd, Harleysville PA 19438

Contact Sandy Wilson

Ph 610.584.6302 Fax 610.584.0586

E-mail sanwilinc@verizon.com

URL http://www.creeksidefarmsnatural

solutions.com

Since 1980. Plants, Seeds.

Let our 32 years of naturalized landscape construction and experience help you choose the right plant for the right place, bringing all of nature's winged wonders into your backyard.

Santa Ana Garden Center

157 Jemez Dam Rd,

Bernalillo NM 87004

Contact Carol Scrivner

Ph 505.867.1322 Fax 505.771.0392

E-mail info@santaana.org

Private, wholesale, retail. Plants. 
Santa Barbara Botanic Garden 1212 Mission Canyon Rd,

Santa Barbara CA 93105

Ph 805.682.4726 Fax 805.563.0352

E-mail cbornstein@sbbg.org or

breed@sbbg.org

Private, retail. Plants.

Santa Fe Greenhouse Inc

2904 Rufina St,

Santa Fe NM 87507-2929

Contact David Salman

Ph 877.811.2700; 505.473.2700

E-mail info@santafegreenhouses.com

Private, retail. Plants, Seeds.

Sassafras Farm

7029 Bray Rd, Hayes VA 23072

Contact Denise Greene

Ph 804.642.0923 Fax 804.642.0923

E-mail sassafrasfarm@verizon.net

Private, wholesale, retail. Plants.

Sauvie Island Ornamentals LLC

19708 NW Gillihan Rd,

Portland OR 97231

Ph 503.621.3086 Fax 503.621.1292

E-mail siotrees@yahoo.com

Private, wholesale. Plants.

Scholls Valley Native Nursery LLC PO Box 231088, Tigard OR 97281

Contact Len Norwitz

Ph 503.624.1766 Fax 503.624.2766

E-mail SVNNLen@aol.com

Private, wholesale. Plants.

SCHUMACHERS NURSERY \&

BERRY FARM INC

37806 910th St,

Heron Lake MN 56137

Contact Judy Schumacher

Ph 507.793.2288 Fax 507.793.0025

E-mail berry01@centurytel.net

URL http://www.schumachers

nursery.com

Private, wholesale, M-F 8-5, since 1966.

Plants: $75 \%$ native, $100 \%$ propagated; container, bareroot, cuttings, bioengineering;

tree, shrub, wetland/aquatic.

Producing high quality deciduous and conifer seedlings and transplants for more than 40 years.
Scioto Gardens

3351 State Rt 37 W,

Delaware OH 43015

Contact Michael Johnson

Ph 740.363.8264 Fax 740.363.8264

Private, wholesale, retail. Plants.

\section{THE SCOTTS MIRACLE-GRO}

\section{COMPANY}

14111 Scottslawn Rd,

Marysville OH 43041

Contact Keri Butler

Ph 937.644.0011

E-mail keri.butler@scotts.com

URL http://www.scotts.com

Services.

The Scotts Miracle-Gro Company, through its wholly owned subsidiary, The Scotts Company $L L C$, is the world's largest marketer of branded consumer products for lawn and garden care, with products for professional horticulture as well. In the US, the Company's Scotts ${ }^{T M}$, Miracle-Gro ${ }^{T M}$, and Ortho ${ }^{T M}$ brands are market-leading in their categories, as is the consumer Roundup ${ }^{T M}$ brand, which is marketed in North America and most of Europe exclusively by Scotts and owned by Monsanto. The Company also owns Smith \& Hawken, a leading brand of garden-inspired products that includes pottery, watering equipment, gardening tools, outdoor furniture, and live goods. Additionally, the Company owns Scotts Lawn Service, a provider of residential lawn care, and Morning Song ${ }^{T M}$, a leading brand in the wild bird food category.

Sebastian River Ranch LLC 5955 82nd Ave, Vero Beach FL 32966 Contact Tom Hammond

Ph 772.778.6560 Fax 772.778.6532

Private, wholesale, retail. Plants.

SECOND GROWTH INC

PO Box 11080, Eugene OR 97440

94739 Turnbow Ln,

Junction City OR 97448

Contact Jean Fogden or Juan Welsh

Ph 541.485.3250; 541.998.1886

Fax 541.345.4481

E-mail jeanfogden@efn.org or

secondgrowthinc@qwest.net

Private, wholesale, retail, contract, by appt (541.998.1886), since 1992. Plants: 98\% native, $90 \%$ propagated, $2 \%$ wild collected.

I grow approximately 80 species native to the Pacific Northwest. All my plants are grown in containers, mostly propagated in small cells, then transplanted. Contract growing available.
Seed Specialists Inc

2723 S Cole Rd, Boise ID 83709

Contact Dan Macias

Ph 208.562.0479 Fax 208.562.0479

E-mail dantheseedman@aol.com

Private. Seeds.

SeedClean

8120 Blaikie Court, Sarasota FL 34240

Contact Gil Sharell

Ph 941.378.3540; 941.378.2700

Fax 941.378.0020

E-mailinfo@seedclean.com

Private, wholesale, mail, contract. Plants, Seeds.

Seedhunt

PO Box 96, Freedom CA 95019-0096

Contact Virginia Hunt

Fax 831.728.5131

E-mail seedhunt@cruzio.com

Private, retail, mail. Seeds.

Seeds of Alaska

PO Box 3127, 48535 Cardwell Rd,

Kenai AK 99611-3127

Contact Dick

Ph 907.262.5267; 907.260.1980

Fax 907.260.1977

Private, wholesale, retail. Plants, Seeds.

\section{SEEDS TRUST}

HIGH ALTITUDE GARDENS

PO Box 596, 9255 Loya Ln,

Cornville AZ 86325

Contact Bill McDormand

Ph 928.649.3315 Fax 928.649.8181

E-mail support2@seedstrust.com

URL http://www.seedstrust.com

Private, wholesale, retail, mail, Internet, contract, by appt, since 1984 . Seeds: $50 \%$ native, $40 \%$ propagated, $40 \%$ wild collected; shrub, herbaceous, grass.

25 years old, family owned. Seeds adapted to cold climates.

We encourage and teach seed saving. We collect wildflower seeds in an environmentally sensitive and sustainable manner. We have taken the safe seed pledge. We do not buy, sell, or use genetically modified seeds. Everything we sell comes with our complete guarantee. 
Seeds West Garden Seeds

157 Jemez Dam Rd, Bernalillo NM 87004

Ph 505.867.1322 Fax 505.867.3395

Wholesale, retail, contract. Seeds.

Seneca Hill Nursery

3712 Co Rt 57, Oswego NY 13126

Contact Ellen Hornig

Ph 315.342.5915 Fax 315.342.5573

E-mail hornig@earthlink.net

Private, retail. Plants.

\section{SEVENOAKS NATIVE NURSERY LLC}

29730 Harvest Dr SW, Albany OR 97321

Contact Mike Ridling or Scott Anderson

Ph 541.757.6520 Fax 541.738.2607

E-mail info@sevenoaksnativenursery.com

URL http://www.sevenoaksnativenursery.com

Private, wholesale, contract, M-Sat 8-4:30, since 1979. Plants:

$5,000,000 / y, 99 \%$ native, $98 \%$ propagated, $0 \%$ wild collected; con-

tainer, bareroot, cuttings, bioengineering; tree, shrub, herbaceous,

grass, wetland/aquatic. Seeds: $99 \%$ native, $98 \%$ propagated; tree,

shrub, herbaceous. Min: $\$ 200$.

Wholesale grower of more than 150 species native to the Western United States. 98 percent grown from sourced seed. Bareroot, 1-20 gal. Quaking aspen specialists.

SGS MID-WEST SEED SERVICES INC

236 32nd Ave, Brookings SD 57006

Contact Laura Carlson RST

Ph 605.692.7611; 877.692.7611

Fax 605.692.7617 E-mail us.seedinfo@sgs.com

URL http://www.seedservices.sgs.com

Private, M-F 8-5, since 1993. Services.

Seed-testing lab.

SGS Mid-West Seed Services Inc offers complete seed-testing services.

Same day planting year-round, website results. Visit our website today.

See ad on page 215 .

Shadowlawn Nursery

PO Box 515, 2729 Co Rd 218,

Penney Farms FL 32079-0515

Contact Brent Reeves

Ph $904.269 .5857 \times 305$

Fax 904.269.5857

E-mail jbryan@shadowlawnnursery.net

Private, wholesale. Plants.

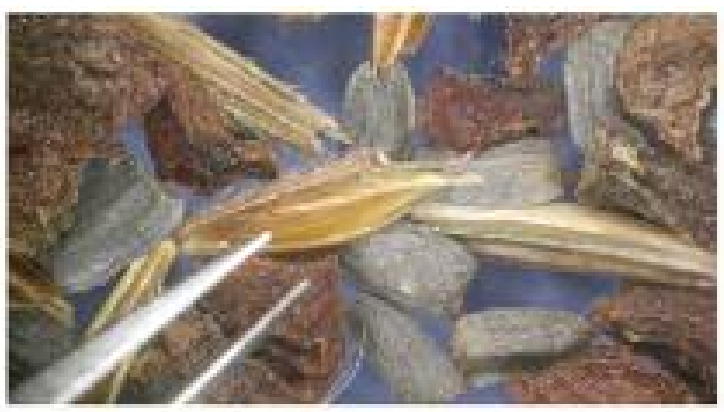

DON'T GAMBLE ON THE LAST STEP... YOUR SEED QUALITY NATIVE SEED TESTING SERVICES

- ACCURATE - 6 FSTs working on natives

- EXPERIENCED - mote than 300 soccies bested

- FAST - sarma-day planting

- INNOVATIVE - real-time vebtsite resulis as comp eted

\author{
SGS Mivss Brookings, USA \\ 236 3znd Are. Broukings. \\ South Dawota. USA b7006 \\ E-1 (605) 652-7อT \\ $\mathrm{F} ;+1$ i $6 \mathrm{C} 5$ ! $892781 \mathrm{Y}$ \\ Emsil: lours carlsonei sos.com \\ uruw, secebservices sgs com
}

WHeN You NeEO To He Buns

Shady Creek Native Plants and

Environmental Consulting

PO Box 491, Abbotsford BC V2T $6 Z 7$

1806 Clearbrook Rd, Abbotsford BC V2T 5X4

Contact Myles Hargrove

Ph 604.856.2010 Fax 604.746.2011

E-mailinfo@shadycreek.ca

Private, wholesale, retail. Plants.

Shady Grove Plantation Nursery

3030 Charleston Hwy, Orangeburg SC 29115

Contact Jeb

Ph 803.534.5683 Fax 803.534.5699

E-mail shadygrove@sc.rr.com

Private, wholesale. Plants.

Sharp Bros Seed Company

Box 140, 1005 S Sycamore, Healy KS 67850

Contact Eric Woofter

Ph 620.398.2231; 800.462.8483 Fax 620.398.2220

E-mail eric@sharpseed.com

Private, wholesale, retail, mail. Seeds.

Sharp Bros Seed Company of Missouri

396 SW Davis-Ladue, Clinton MO 64735

Ph 660.885.7551 Fax 660.885.8647

E-mail sales@sharpbro.com

Private, wholesale, retail, mail. Seeds. 
Shasta Plantation

PO Box 535, Shasta CA 96087

Ph 530.243.8001 Fax 530.243.8001

E-mail plantation@shasta.com

Private, wholesale. Plants.

Shelterwood Farm

179 Tuxward Rd, Hartly DE 19953

Contact John Schwalm

Ph 302.492.8071

Private, wholesale. Plants.

Sheltherbelt Builders Inc

3088 Claremont Ave,

Berkeley CA 94705

Ph 510.841.0911 Fax 510.601.6896

Private, wholesale, retail. Plants.

SHOOTING STAR NATIVE SEEDS

PO Box 648, 20740 Co Rd 33, Spring

Grove MN 55947

Contact Mark Udstuen

Ph 507.498.3944 Fax 507.498.3953

E-mail

ssns@shootingstarnativeseeds.com

URL http://www.shootingstarna

tiveseed.com

Private, wholesale, M-F, since 1989. Seeds:

$100 \%$ native.

See ad on page 217.

Shooting Star Nursery

160 Soards Rd, Georgetown KY 40324

Contact Mary Ann Hunt

Ph 502.867.7979 Fax 502.867.7677

E-mail shootingstarnursery@msn.com

Private, retail, mail. Plants.

Sierra Seed Supply

358 Williams Valley Rd,

Greenville CA 95947

Ph 530.284.7926

Plants.

Sierra Valley Farms

PO Box 791329 Co Rd A-23,

Beckwourth CA 96129

Contact Gary Romano

Ph 530.832.0114 Fax 530.832.5114

E-mailsvfarms@plumas.com

Wholesale, retail, contract. Plants.
Sierra Vista Growers

PO Box 225, 2800 NM Hwy 28,

La Union NM 88027

Contact Kevin Padilla

Ph 505.874.2415 Fax 505.589.3024

E-mailsvwg@netzero.net

Private, wholesale, retail. Plants.

Silk Tree Gardens

65 Harlem Ave, Bridgeport CT 06606

Ph 203.870.6213 Fax 203.549.0420

Private, wholesale, retail. Plants.

Silvaseed Company Inc

PO Box 118, 317 James St,

Roy WA 98580

Contact Mike Gerdes

Ph 253.843.2246 Fax 253.843.2239

E-mail mikegerdes@silvaseed.com

Forest Industry, wholesale, retail, mail, contract. Plants, Seeds.

Silver Mountain Nursery

4672 Drift Creek Rd SE, 13773 Silver

Falls Hwy SE,

Sublimity OR 97385

Ph 503.769.7133 Fax 503.769.1647

E-mail jimf@silvermtnnursery.com

Private, wholesale. Plants.

Silver Springs Nursery Inc

9609 Sterling Creek Rd, 700 Hamilton

Rd, Jacksonville OR 97530

Contact James Kraemer

Ph 541.899.1065; 541.261.5831

Fax 541.899.1065

E-mail silversprings@wildblue.net

Private, wholesale. Plants.

Simonton Farms Nursery

22159 NW 75th Ave Rd, Micanopy FL

32667

Contact Sid McCarty

Ph 352.591.2271 Fax 352.591.2319

E-mail mccartysrats@yahoo.com

Private, wholesale, retail. Plants.

Simply Native Nursery

681 State Hwy 135, Alexis IL 61412

Contact Kathy Hale-Johnson

Ph 309.371.9598 Fax 309.586.4078

E-mail

contact@simplynativenursery.com

Private, retail, mail. Plants.
Simpson Timber Company-

Korbel Forest Nursery

PO Box 68, Korbel CA 95550

Ph 707.668.4539 Fax 707.668.5196

E-mail glehar@simpson.com

Forest Industry. Plants.

Sinagua Nursery

7800 Rain Valley Rd, Flagstaff AZ

86004-1438

Ph 520.527.1595

Private, wholesale. Plants.

Siskiyou Rare Plant Nursery

2115 Talent Ave, Talent OR 97540

Ph 541.772.6846 Fax 541.535.3112

E-mail customerservice@srpn.net

Private, retail, mail. Plants.

Siskiyou Tree \& Mountain

200 South A St,

Mount Shasta CA 96067

Ph 916.926.4755 Fax 916.926.4755

Private, wholesale, contract. Plants.

Sky Native Plant Nursery

14713 River Bend Rd, Mount Vernon

WA 98273

Contact Nathan 360.661 .4280 or Andy

Ph 360.848.5828; 360.661 .4280

Fax 360.854.7665

E-mail nelanson@pnt.org

Private, wholesale, retail. Plants.

Sleepy Hollow Nursery

3506 Harrison Ferry Rd,

McMinnville TN 37110

Contact George Dodson

Ph 931.668.3902; 931.668.2462

Fax 931.668.2443

E-mail sleepyhollow@blomand.net

Private, wholesale. Plants.

Smith Evergreen Nursery Inc

9240 Bachlor Rd, Magnolia OH 44643

Contact Mike Smith

Ph 330.866.5521 Fax 330.866.5379

E-mail nsmithevergreen@neo.rr.com

Private, wholesale. Plants. 
Smith River Nursery-

Hastings LLC

PO Box 250, Smith River CA 95567

Ph 707.487.3775 Fax707.487.9301 E-mail srtrees@gte.net Private, wholesale, retail, mail. Plants.

Snider Nursery

500 Co Rd 386, Gorman TX 76454

Contact Mike or Crystal

Ph 254.734.2027

Private, wholesale, retail. Plants.

Snow Mountain Nursery

1615 Rosebrook Rd, Standardsville VA 22973

Contact Georgiana McCabe

Ph 434.985.6789 Fax 804.985.6659

Private, wholesale, retail. Plants.

Soaring Eagle Nursery

7321 Howard Rd, Bokeelia FL 33922

Ph 800.293.7256; 239.283.9282 Fax 800.721.5714

Private, wholesale. Plants.

Sound Native Plants Inc

PO Box 7505, Olympia WA 98507-7505

Contact Cathy Sampselle

Ph 360.352.4122 Fax 360.867.0007

E-mail cathy@soundnativeplants.com

Private, wholesale, retail, contract, M-F 9-5, since 1992. Plants:

$90,000 / y, 100 \%$ native, $100 \%$ propagated; container, cuttings, bio-

engineering.

We are dedicated to four main facets of restoration: growing, installing, consulting, and training/education. Call us to discuss your project.

South Carolina Forestry Commission-Taylor Nursery PO Box 219, 53 Girl Scout Camp Rd, Trenton SC 29847

Contact Steve Cantrell

Ph 803.275.3578 Fax 803.275.5227

E-mail taylortree@pbtcomm.net

State, retail. Plants.

Southeast Trees I Bartow Ornamental Nursery 3890 Hwy 60 E, 200 Gandy Cemetery Rd, Bartow FL 33830

Contact Ken Ford

Ph 888.534.1350; 863.534.1350

Fax 863.534.1356

E-mail ken@southeasttrees.com

Private, wholesale. Plants.

\section{Southern Horticulture}

1690 A1A S, St Augustine FL 32080

Contact Bill or Bryanne Hamilton

or Jean Dowdy

Ph 904.471.0440 Fax 904.460.1222

E-mail soho@bellsouth.net

Private, retail, M-Sat 8-5:30; Sun 8-5, since 1979. Plants.

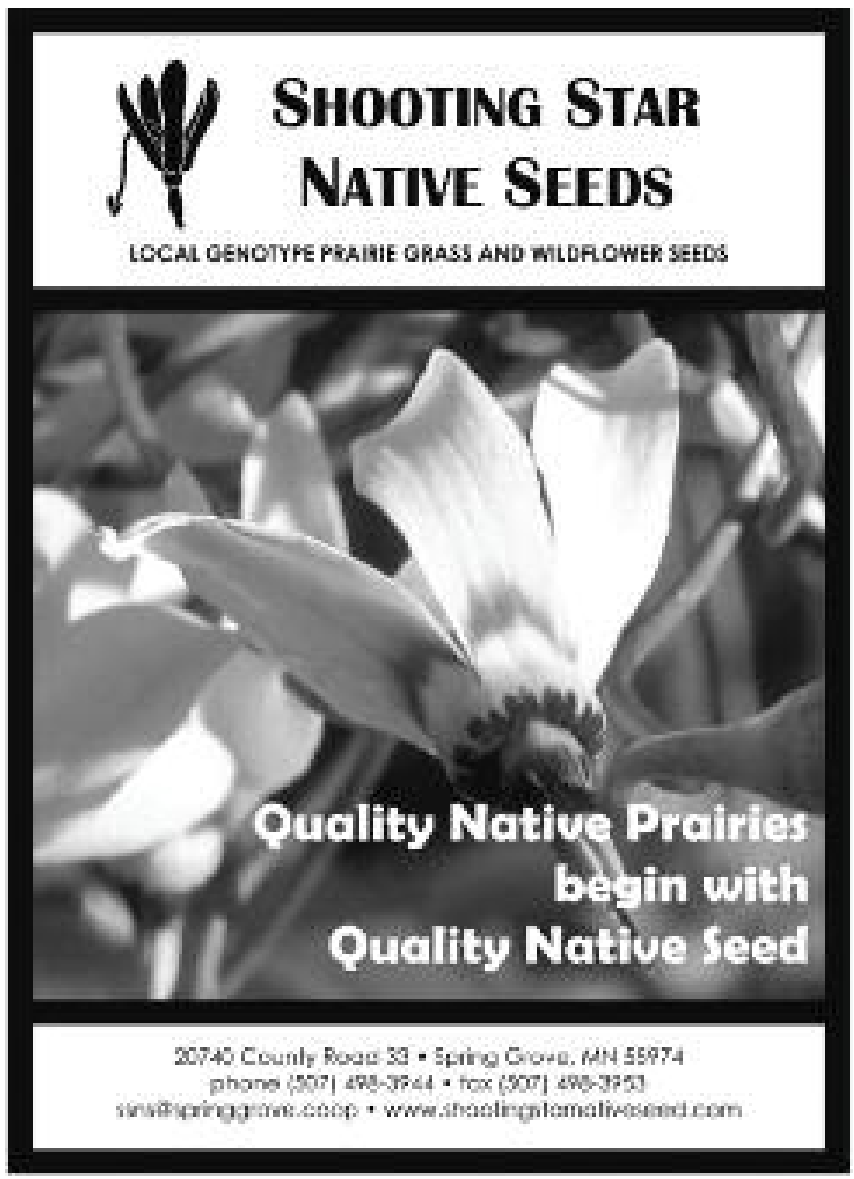

Southern Native Nursery Inc

16351 Van Gogh Rd, Loxahatchee FL 33470

Contact Mike Catron

Ph 561.798.1172 Fax 561.798.2816

E-mail slandnsy@bellsouth.net

Private, wholesale, retail. Plants.

Southern Native Plants LLC

6322 Mary Kitchens Rd, Milton FL 32583

Contact Paul Humbert

Ph 850.983.9121

E-mail sonative@yahoo.com

Private, wholesale, retail. Plants.

Southern Pride Tree Farm Inc

6400 NW 42nd Ct, Bell FL 32619

Contact James Gray

Ph 386.935.3636 Fax 386.935.3722

E-mail jimmy@sptreefarm.com

Private, wholesale. Plants.

\section{Southern Roots Tree Nursery}

448 Daisy Log Rd, Blairsville GA 30512

Contact Barry Gay

Ph 706.835.1662E-mail treesgay@windstream.net

Private, wholesale, retail, contract. Plants. 
Southern Tier Consulting and Nursery Inc

PO Box 30, 2701 A Rt 305, West

Clarksville NY 14786

Contact Johneta Wilson

Ph 585.968.3120 Fax 585.968.3122

E-mail froghome@southerntier

consulting.com

Private, wholesale, retail. Plants.

Southwest Seed Inc

13260 Rd 29, Dolores CO 81323

Contact Roberta Henes

Ph 970.565.8722; 800.543.1279

Fax 970.565.2576

E-mail swseed@southwestseed.com

Private, wholesale, retail, mail, Internet.

Seeds.

Southwestern Native Seeds

PO Box 50503, Tucson AZ 85703

Private, retail, mail.

Seeds.

Spadefoot Nursery

8897 E Walnut Trail, Pearce AZ 85625

Contact Peter

Ph 520.824.3247; 520.909.9919

Fax 520.824.3247

Private, wholesale. Plants.

SPENCE RESTORATION NURSERY

2220 E Fuson Rd, Muncie IN 47302

Contact Kevin Tungesvick

Ph 765.286.7154 Fax 765.286.0264

E-mail kevin@spencenursery.com

URL http://www.spencenursery.com

Private, wholesale, M-F 7-4, since 1995.

Plants, Seeds.

Ohio Valley and southern Great Lakes genotypes of prairie, wetland, and woodland plants and seeds. Plants are mycorrhizal inoculated in open-bottomed pots.

Spring Creek Nursery

3226 W Montgomery Rd,

Deer Park WA 99006

Contact Fitzgerald

Ph 509.276.8278 Fax 509.276.8302

E-mail Grower4u@aol.com

Private, wholesale. Plants.
Spring Lake Restoration Nurseries

21938 Mushtown Rd,

Prior Lake MN 55372

Contact Corrine Daniels

Ph 952.447.1919 Fax 952.447.1920

E-mail slrn@appliedeco.com

Private, wholesale, contract. Plants, Seeds.

Springbrook Farms

322 Chagebridge Rd,

Pine Brook NJ 07058

Ph 973.227.1007

E-mailinfo@chuxlandscaping.com

Private, retail. Plants.

St Lawrence Nurseries

325 State Hwy 345, Potsdam-Madrid

Rd, Potsdam NY 13676

Ph 315.265.6739

E-mail trees@sIn.potsdam.ny.us

Private, wholesale, retail, mail. Plants.

Star Nurseries

PO Box 79, 1329 Co Rd A-23,

Beckwourth CA 96129

Ph 530.932.0114 Fax 530.832.5114

E-mailsvfarms@plumas.com

Private. Plants.

Star Nursery

1145 W Sunset Blvd,

St George UT 84770

$\mathrm{Ph}$ 435.673.0820

Private, wholesale, retail. Plants.

Stempky Nursery

5157 N Straits Hwy,

Cheboygan MI 49721

Contact Fred or Susan Stempky

Ph 231.627.4814 Fax 231.627.3087

Private, wholesale. Plants.

Stevenson Intermountain Seed

PO Box 2, 488 S $1090 \mathrm{E}$,

Ephraim UT 84627

Contact Ron Stevenson

Ph 435.283.6639 Fax 435.283.4155

E-mail roon@stevensonintermountain seed.com

Private, wholesale, retail. 8-5, since 1976.

Seeds: $90 \%$ native.
Stillaguamish Tribe Bank Savers

PO Box 277, 22712 6th Ave NE,

Arlington WA 98223-0277

Contact Doug Kinfield; John Drotts, Natural Resource Manager $\times 26$; Pat Stevenson, Environmental Manager $\times 27$ Ph 360.435.2755; 360.435 .9365

Fax 360.435.3605

E-mail dkinfield@stillaguamish.nsn.us Tribal, wholesale. Plants, Seeds.

Stock Seed Farms

28008 Mill Rd, Murdock NE 68407

Contact Rod Fritz

Ph 800.759.1520 Fax 402.867.2442

E-mail rod@stockseed.com

Private, wholesale, retail, mail. Seeds.

Stone Silo Prairie Gardens

4500 Oak Ridge Circle, 2325 Oak Ridge

Circle, DePere WI 54115

Contact Pat or Ceci Kiefer

Ph 920.336.1662 Fax 920.336.5745

E-mailinfo@stonesiloprairiegardens.com

Private, wholesale, retail. Plants.

Storm Lake Growers

18510 SR 203,

Monroe WA 98272-8801

Contact Terra Sittner or Dan McCain

Ph 360.794.4842 Fax 360.794.8323

E-mail terra@slgrowers.com

Private, wholesale. Plants.

Stover Seed Company

PO Box 21488, 1415 E 6th St,

Los Angeles CA 90021

Contact Stephen Knutson

Ph 213.626.9668 Fax 213.626.4920

E-mail Stephen_k@stoverseed.com

Private, wholesale, M-F 8-5, since 1922.

Seeds: $5 \%$ native. Min: $\$ 150$.

Custom collection, blending/mixing.

Strathmeyer Forests Inc

PO Box 70, 255 Ziegler Rd, Dover PA 17315

Ph 800.345.3406 Fax 717.292.4129

E-mail sales@strathmeyer.com

Private, wholesale. Plants. 
Strawberry Plains Audubon Center

285 Plains Rd, Holly Springs MS 38635

Contact Kristin Lamberson

Ph 662.252.1155 Fax 662.252.8844

Private, retail. Plants.

Streamside Native Plants

7455 Island Hwy W, 3222 Grant Rd, Bowser BC V0R 1G0

Contact Richard Wahlgren

Ph 250.757.9999 Fax 250.757.9999

E-mail richard@streamsidenativeplants.com

Private, wholesale, retail. Plants.

Stuewe \& Sons Inc

31933 Rolland Dr, Tangent OR 97389

Contact Eric Stuewe

Ph 541.757.7798; 800.553.5331

Fax 541.754.6617

E-mailinfo@stuewe.com

Private, wholesale. Services.

We sell nursery growing containers used for propagating and growing tree seedlings. These nursery trays and pots are uniquely designed for optimum seedling development and root enhancement. We can also develop new designs to your specifications. Our nursery containers are used by many native and restoration nurseries across the US.

See ad on page 219 .

Sturon Inc

15801 SW 216th St, Miami FL 33170

Contact Steven C Leonard

Ph 305.232.9005 Fax 305.233.7060

Private, wholesale. Plants.

Sugarbush Nursery

4272 Morgantown Rd, Mohnton PA 19540

Ph 610.856.0998 Fax 610.856.0998

E-mailinfo@sugarbushnursery.com

Private, retail. Plants.

Summer Hill Nursery

888 Summerhill Rd, Madison CT 06443

Contact Mike Johnson Ph 203.421.3055 Fax 203.421.5189

Private, wholesale. Plants.

Summit Nursery

5432 Summit St, Centreville VA 20120

$\mathrm{Ph} 703.830 .2656$

Private. Plants.

\section{Helping you grow...}

\section{with containers for native plant production}

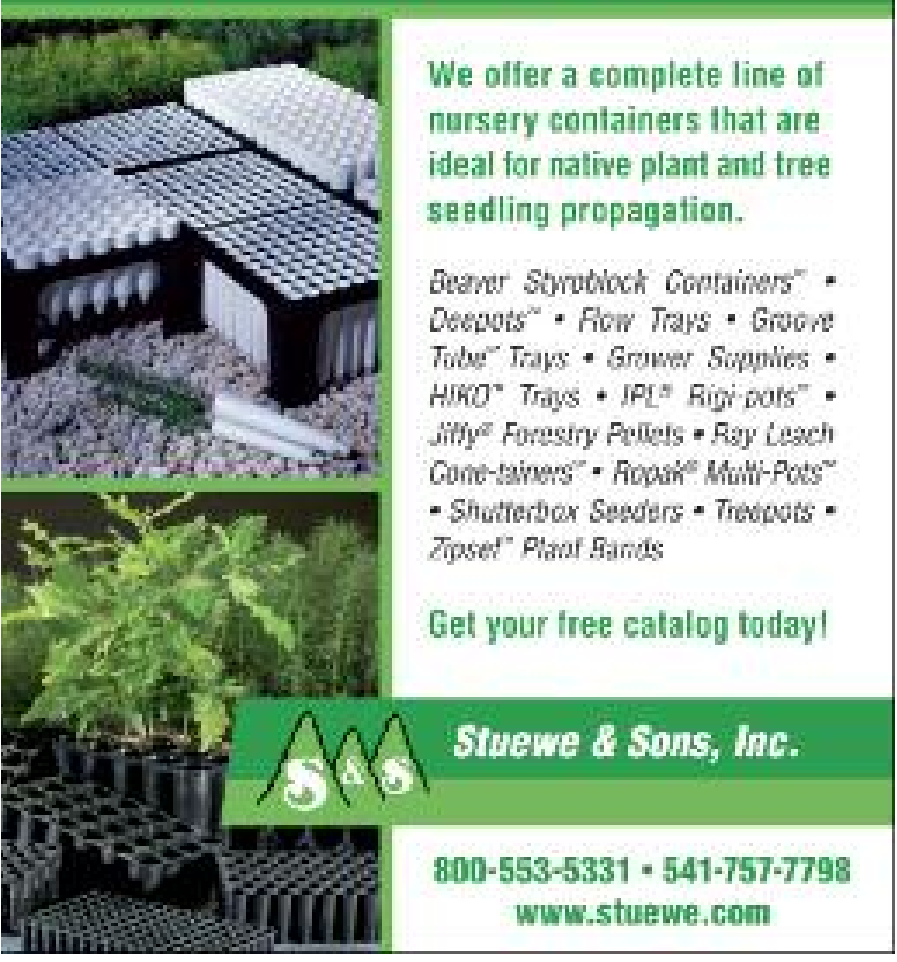

directory key

NATIVE PLANTS-R-US

123 Main Street, Anytown ID 83843

Contact John Q Public

Ph 800.555.1212 Fax 800.555.1212

BUSINESS
TYPE -mail plants-r-us@somewhere.com

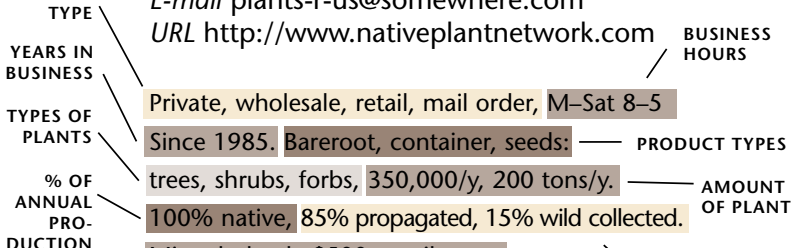

DUCTION
IS NATIVE

MINIMUM $/$

on-line catalog and ordering.

See ad on page 45 - VIEW THEIR AD

$\%$ OF ANNUAL

PRODUCTION THAT IS

PROPAGATED AND (OR)

ORDER

OTHER We offer more than 600 species of plants native to

SERVICES the northern Rocky Mountains, specializing in locally

OFFERED collected, source-identified Palouse Prairie plants. Our staff can help you plan your restoration project, facilitate collection and amplification of local seed sources, and supervise the restoration.

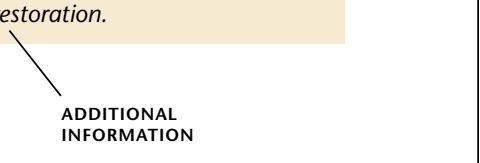


SUN GRO HORTICULTURAL

DISTRIBUTION INC

19287 Hwy 99 E, Bellevue WA 98008

Contact Jason Padden

Ph 503.729.0655 Fax 425.212.0153

E-mail jason@sungro.com

URL http://www.sungro.com

Private, wholesale, retail. Services.

See ad on page 221.

Sun Mountain Growers

PO Box 332, 336 N Mountain Rd,

Kaysville UT 84037

Contact Brad Soelberg

Ph 801.941.5535 Fax 801.529.1210

E-mailsunmtngrowers@comcast.net

Private, wholesale, retail, M-F 8-4:30, Sat by appt, since 2000. Plants: $100,000 / y, 80 \%$

native, $90 \%$ propagated; container, cuttings.

\section{Sun Mountain Natives}

1406 East F St, Moscow ID 83843

Contact Randy Gilmore

Ph 208.883.7611 Fax 208.882.6738

E-mail rgilmore@turbonet.com

Private, wholesale, $8-5$, since 1994 . Seeds:

$90 \%$ native, $20 \%$ propagated, $80 \%$ wild collected.

Seed company specializing in hand-collected natives with a Northwest origin. We offer custom cleaning and collection.

Sunbelt Trees Inc 16008 Boss Gaston, Richmond TX 77469

Ph 800.625.4313

Private, wholesale. Plants.

\section{SUNBREAK NURSERY COMPANY}

5192 Aldrich Rd, Bellingham WA 98226 Contact Rick Wright or Ryan Carpenter Ph 360.384.3763 Fax 360.384.5030

E-mail sunbreaknurserycompany@

comcast.net

URL www.sunbreaknursery.com

Private, wholesale, retail, M-F 9-4:30, since

1978. Plants: $20 \%$ native, $75 \%$ propagated.

We specialize in ground covers and ferns for the retail and landscape markets. We offer free weekly deliveries to most Puget Sound locations.

\section{SUNCO}

2269 2nd Ave N, Lake Worth FL 33461

Contact Jerry Fritz

Ph 561.309.2772 Fax 561.588.9486

E-mail suncojerry@aol.com

Private, wholesale. Plants.
Suncrest Nurseries Inc

400 Casserly Rd, Watsonville CA 95076

Contact Victor Quintero

Ph 831.728.2595 Fax 831.728.3146

Private, wholesale. Plants.

Sundance Ornamentals

10689 Heritage Blvd,

Lake Worth FL 33467-6723

Ph 561.965.1344 Fax 561.965.2236

E-mail ymir3@ix.netcom.com

Private, wholesale, contract. Plants.

Sunflower Farms

Rt 2 Box 26AA, 1065 S Olive St, Cherryvale KS 67335

Contact Mark or Sandy

Ph 620.336.2066 Fax 620.336.2432

E-mail sunflowerfarms@sbcglobal.net Private, wholesale, retail. Plants.

Sunlight Gardens Inc

174 Golden Ln,

Andersonville TN 37705

Contact Andrea Sessions

Ph 865.494.8237 Fax 865.494.7086

E-mail info@sunlightgardens.com

Private, wholesale, retail, mail, Internet.

Plants.

Sunmark Seeds International Inc

906 NW Corporate Dr,

Troutdale OR 97060

Contact Robin Cook

Ph 888.241.7333

E-mail natives@sunmarkseeds.com

Private, wholesale, mail, M-F 8-5, since

1983. Seeds: $20 \%$ native, $80 \%$ propagated.

Sunny Border Nurseries Inc PO Box 216, 1709 Kensington Rd, Kensington CT 06037

Contact Steve or Mark

Ph 800.732.1627 Fax 860.828.9318

E-mail sales@sunnyborder.com

Private, wholesale. Plants.

Sunrise Nursery

73-4939 Kamanu St, Kailua-Kona HI 96740

Contact Margo Lundstrom

Ph 808.329.7593 Fax 808.325.6249

Private, wholesale, retail, contract. Plants.
Sunscapes

330 Carlile Ave, Pueblo CO 81004

Contact Bill Adams

Ph 719.546.0047 Fax 719.546.0047

E-mail sunscapes@comcast.net

Private, retail. Plants.

Sunset Coast Nursery

2745 Tierra Way, Aromas CA 95004

Contact Patti Kreiberg

Ph 831.726.1672 Fax 831.726.1672

Private, wholesale, retail, contract, M-F 8-4, since 1984. Plants: $3715 / y, 100 \%$ native, $100 \%$ propagated; container, bareroot.

Specializing in CA natives. We offer site-specific services: consult, design, seed collection, contract growing.

SUNSHINE FARM AND GARDENS

HC67 Box 539B, Renick WV 24966

Contact Barry Glick

Ph 304.497.2208 Fax 304.497.2698

E-mail barry@sunfarm.com

URL http://www.sunfarm.com

Private, wholesale, retail, by appt, since

1972. Plants, Seeds.

Nursery-grown unusual native plants.

See ad on page 223.

Sunshine Gardens Nursery and

Landscaping Inc

1286 Shadywood Shores Dr NW,

Pine River MN 56474

Contact Bonnie Hiniker

Ph 218.947.3154; 218.821.8671

E-mailsgardens@uslink.net

Private, wholesale, retail. Plants.

Sunshine Nursery

21168 Stick Ross Mountain Rd,

Tahlequah OK 74464

Contact Raymond or Helen Hendrix

Ph 918.456.6927 Fax 918.456.6927

Private, wholesale, retail. Plants.

Superior Trees

PO Drawer 9400, 12493 US Hwy 90 E, Lee FL 32059

Contact Amy Webb or Summer Jones

Ph 850.971.5159 Fax 850.971.5416

Private, wholesale. Plants. 


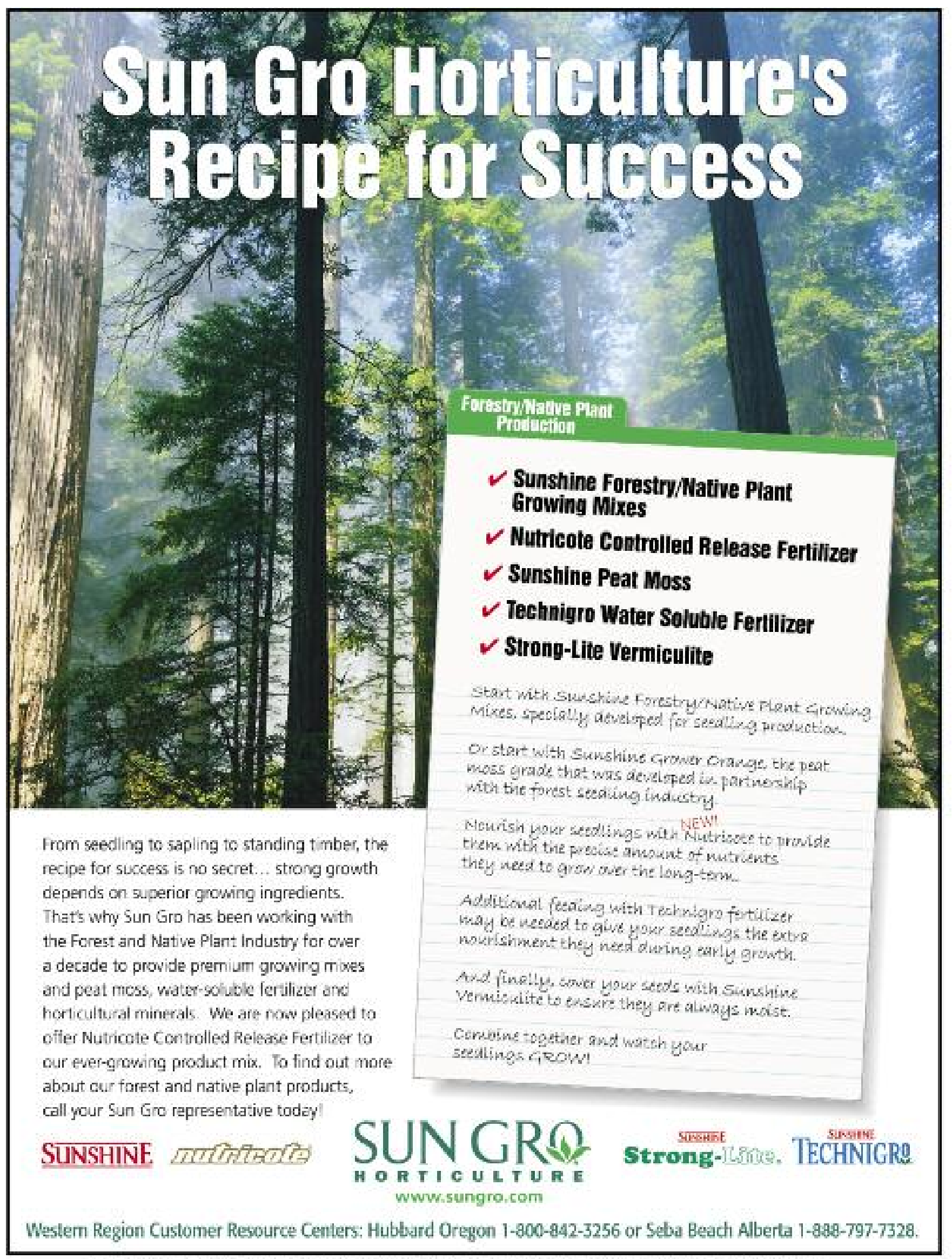


Swanson Farms

64905 190th St, Nevada IA 50201

Contact Andy

Ph 515.382.6120 E-mail andy@mid-

iowa.net

Private, wholesale, retail. Seeds.

Swansons Nursery

9701 15th Ave NW, Seattle WA 98117

Contact Gabriel Maki

Ph 206.782.2543 Fax 206.782.8942

E-mail gabrielm@swansonsnursery.com

Private, retail. Plants.

The Sweetbriar

PO Box 25, Woodinville WA 98072

Ph 425.821.2222 Fax 425.821.2986

Private. Plants.

\section{SYLVA NATIVE NURSERY \& SEED} COMPANY

3815 Roser Rd, Glen Rock PA 17327

Contact Michael Hollins

Ph 717.227.0486 Fax 717.227.0484

E-mailsylvanat@aol.com

URL http://www.sylvanative.com/

Private, wholesale, contract, M-F 8-4:30,

since 1988. Plants: 500,000/y, 100\% native, $98 \%$ propagated, $2 \%$ wild collected; con-

tainer, bareroot, $B \& B$, cuttings, bioengineer-

ing; tree, shrub, herbaceous, grass, wet-

land/aquatic. Seeds: grass.

Native trees, shrubs, vines, grasses and forbs for forest wetland and meadows of the

Northeast, Mid-Atlantic and Southeast US.

Sylvan Options

PO Box 506, Dillard OR 97432

Contact Jim Carlson

Ph 541.679.3161

E-mail sylvan@rosenet.net

Private, wholesale, retail. Plants.

Sylvania Native

Ira Way, Pittsburgh PA 15217

4154 Winterburn Ave,

Pittsburgh PA 15207

Contact Kathy McGregor

Ph 412.421.8551 Fax 412.421.5462

E-mailsylvanianatives@aol.com

Private, retail. Plants.
SYMBIOS RESEARCH AND

\section{RESTORATION}

277 Burden St,

Prince George BC V2M 2H2

Contact Carla Burton

Ph 250.564.9895 Fax 250.564.9895

E-mailsymbios@shaw.ca

URL http://www.bulkley.net/ symbios

Private, retail, mail, Internet, since 1995.

Seeds: $100 \%$ native, $90 \%$ propagated, $10 \%$

wild collected; shrub, herbaceous, grass.

Synergy Resource Solutions Inc

5393 Hamm Rd, Bozeman MT 59715

Contact Jack Alexander

Ph 406.586.4727 Fax 406.585.4729

E-mail synergy@countgrass.com

Private, retail. Services.

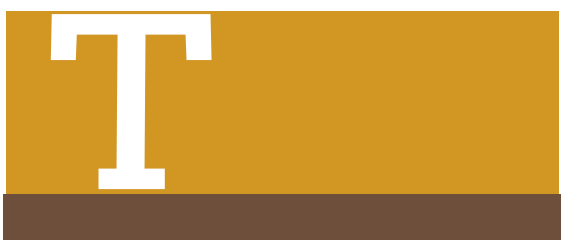

Tadpole Haven Native Plants

20322 197th Ave NE,

Woodinville WA 98072

Contact Shirley Doolittle-Egerdahl

Ph 425.788.6100 Fax 425.844.2824

E-mail tadpole_haven@verizon.net

Private, wholesale, retail, contract, by appt

please call, since 1998. Plants: $100 \%$ native,

$100 \%$ propagated; container.

Tagawa Garden Center \& Florist 7711 S Parker Rd, Aurora CO 80016 Contact Nancy

Ph 303.690.4722 Fax 303.690.9107

Private, retail. Plants.

Tahoe Tree Company

PO Box 5325, 401 Westlake Blvd,

Tahoe City CA 96145

Ph 916.583.3911 Fax 530.583.0557

E-mail leslie@tahoetree.com

Wholesale, retail, contract. Plants.

Tamarack Farms

Box 366, Erickson MB R0J OPO

Contact Harvey Pengelly

Ph 204.636.2990 Fax 204.636.2225

E-mail tamarack@erickson.mb.com

Private, wholesale. Seeds.
Tarweed Native Plant Nursery

\& Landscape

23539 Knapp Way,

Chatsworth CA 91311

Ph 818.888.2318

Private, retail. Plants.

Tarweed Nursery and Landscape

4250 Dundee Dr,

Los Angeles CA 90027

Ph 323.663.0113

E-mail

tarweed@tarweednativeplants.com

Private, wholesale, retail, contract. Plants.

Taylor Creek Restoration

Nurseries

PO Box 256, 17921 W Smith Rd,

Brodhead WI 53520

Ph 608.897.8641 Fax 608.897.2044

E-mail tcrn@appliedeco.com

Private, wholesale, retail, mail, contract.

Plants, Seeds.

Telos Rare Bulbs

PO Box 4147, Arcata CA 95518

E-mail rarebulbs2@earthlink.net

Private, wholesale, mail. Plants.

Temecula Band of the

Luiseno Indians

PO Box 2183, Temecula CA 92593

Contact William Pink

Ph 951.308.9295 Fax 951.506.9491

E-mail wjpink@ivic.net

Tribal, wholesale. Plants.

Tennessee Dept of Agriculture-

Delano Nursery

PO Box 59, 9063 Hwy 411 S,

Delano TN 37325

Contact John Conn

Ph 423.263.1626 Fax 423.263.9322

E-mail john.conn@tn.gov

State, wholesale, retail, contract. Plants.

Texas Forest Service-West Texas

Nursery

Route 3, Box 216, Lubbock TX 79401

Ph 806.892.3572 Fax 806.892.3572

E-mail rfewin@tfs.tamu.edu

State, wholesale, retail. Plants. 


\section{Nursery Propagated Native Plants}

\section{WWW.SUNFARM.COM}

\section{Renick, West Virginia, USA 24966 304.497.2208 - FAX 304.497.3036}

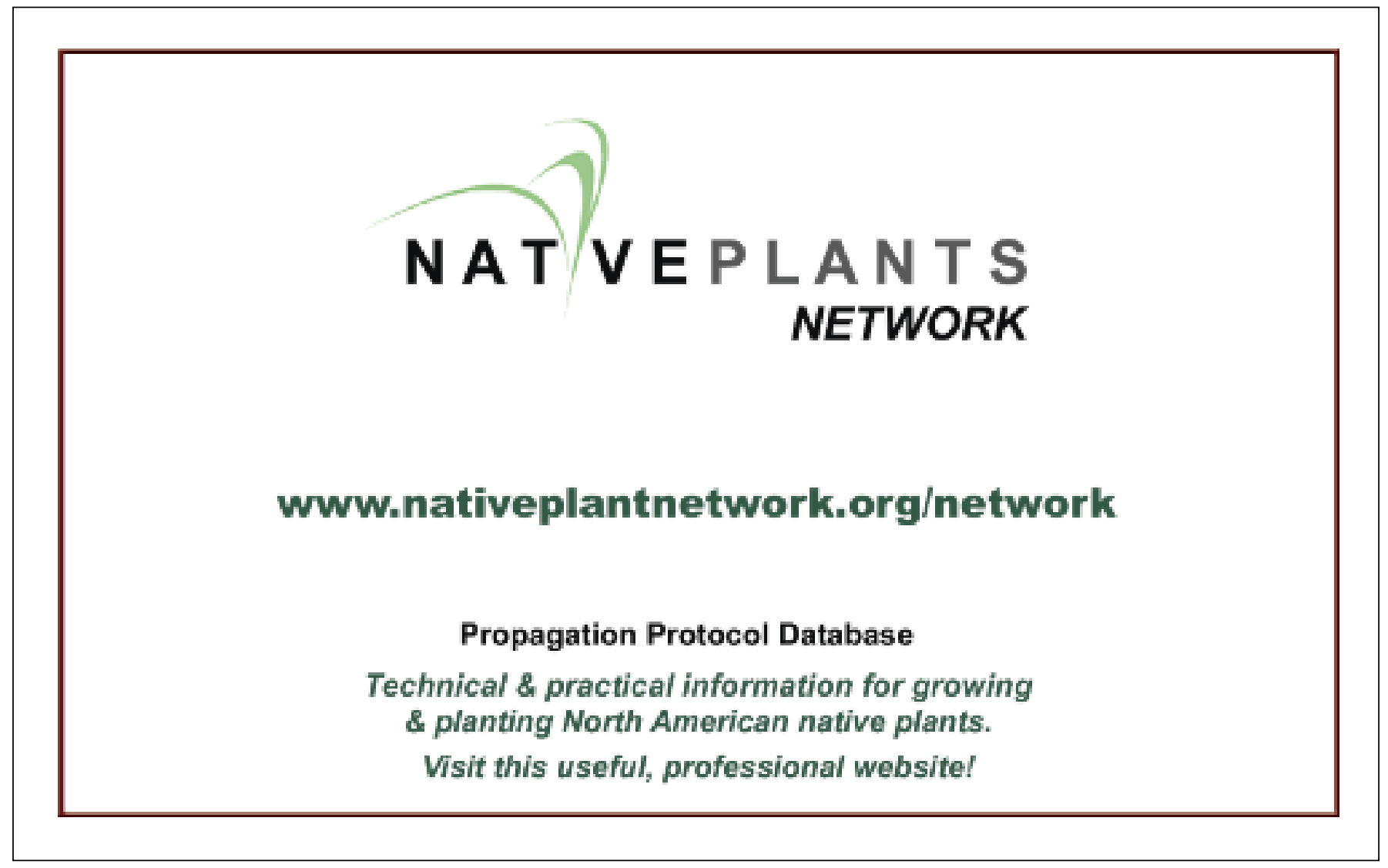


Texas Grown Plants

9114 Hwy 290 W, Austin TX 78736

Contact Terry

Ph 512.288.0806 Fax 512.288.2164

E-mail t.j.hedrick@sbcglobal.net

Private, wholesale, retail. Plants.

Texas Native Trees

PO Box 817, 1006 Glass Dr, Leander TX 78646

Ph 512.260.1697

Private, wholesale, mail. Plants.

Theodore Payne Foundation

Nursery

10459 Tuxford St,

Sun Valley CA 91352-2126

Contact Louise Gonzalez

Ph 818.768.1802 Fax 818.768.5215

E-mail info@theodorepayne.org

Nonprofit, retail. Plants, Seeds.

\section{Thompson \&}

Morgan Seedsmen Inc

PO Box 4086, Lawrenceburg IN 47025

220 Faraday Ave, Jackson NJ 08527

Ph 800.274.7333 Fax 888.466.4769

Private, wholesale, retail, mail, Internet.

Seeds.

\section{THORN CREEK NATIVE}

\section{SEED FARM}

1461 Thorn Creek Rd,

Genesee ID 83832

Contact Jacie or Wayne Jensen

Ph 208.596.9122

E-mail jacie@nativeseedfarm.com

URL http://www.nativeseedfarm.com

Private, wholesale, retail, mail, Internet, please call, since 2004 . Seeds: $100 \%$ native, $100 \%$ propagated; herbaceous, grass. Min: $\$ 10$.

Three-generation family farm specializing in natives since 2004.

Specialize in native upland prairie wildflower seeds that are sourced from the Palouse Prairie in northern Idaho and adapted to the Inland Northwest prairies of Idaho, Washington, Oregon, Montana, and Wyoming. Seeds originate from private land, wild connections, and then are grown and harvested in our fields under thier natural conditions. Certified seed available.
Tidewater Growers

31185 Keller Pond Rd, Keller VA 23401

Ph 757.787.4079 Fax 757.787.4079

Private, wholesale. Plants.

Tiedemann Nursery

PO Box 926, 4707 Cherryvale Ave, Soquel CA 95073

Contact Steve or Julie

Ph 831.475.5163 Fax 831.475.4067

Private, wholesale. Plants.

Tipi Mountain Native Plants

PO Box 946, 8425 Mission Rd,

Cranbrook BC V1C 4J6

Contact Jason Meuleman

Ph 250.427.7010 Fax 250.427.7012

E-mail jason@hotmail.com

Private, wholesale, retail, contract. Plants.

TN Nursery Company

12845 State Rt 108,

Altamont TN 37301

Contact Tammy

Ph 931.692.4252 Fax 931.692.4266

E-mail sales@tnnursery.net

Private, wholesale. Plants.

Toadshade Wildflower Farm

53 Everittstown Rd,

Frenchtown NJ 08825

Contact Randi Eckel

Ph 908.996.7500 Fax 908.996.7500

E-mail toadshad@toadshade.com

Private, retail, mail, Internet, by appt, since

1996. Plants: $100 \%$ native, $100 \%$ propagated.

TODD VALLEY FARMS

PO Box 202, 950 Co Rd M, Hwy 92,

Mead NE 68041

Contact Wayne Thorson

Ph 402.624.6385 Fax 402.624.2003

E-mail info@toddvalleyfarms.com

URL http://www.toddvalleyfarms.com

Private, wholesale, retail, mail, Internet, contract, 7-5, since 1973. Plants: $20 \%$ native, $20 \%$ propagated.

East Hwy 92; Grower of turf type buffalo grass, the only turf grass native to the US. Available as accelerated growth plugs, shipped nationally via UPS or COD.
TOM DODD NURSERY

PO Drawer 45, 9300 Wulff Rd,

Semmes AL 36575

Contact Grady Holt

Ph 334.649.1960

E-mail grady.holt@tomdodd.com or tdn@zebra.net

URL http://www.tomdodd.com

Private, wholesale, M-F 7-4, since 1912.

Plants: $30 \%$ native, $100 \%$ propagated.

Wholesale grower of large-container native ferns and native shrubs such as Azalea, Clethra, Hydrangea, Illicium, Itea, Leucothoe, and Myrica. Native trees such as Magnolia, Pinus, and Taxodium. We ship throughout the Southeast, Northeast, and Midwest. Please contact us for any contract growing needs you have, and check out our website.

Total Resource Management 382 NW Hwy 18, Clinton MO 64735 Contact Jeff

Ph 660.885.6127 Fax 660.885.7152

E-mail trm@prairiesource.com

Private, wholesale, retail. Plants, Seeds.

Trail Creek Nursery Inc

707 S Hwy 33, Victor ID 83455

Contact Jayson Calderwood or

Carolyn Coleman

Ph 800.336.2470; 208.787.2470

Fax 208.787.2475

E-mail jayson@trailcreeknursery.com

Private, retail. Plants.

Trail Ridge Nursery

PO Box 967, 6768 Timberlane Dr,

Keystone Heights FL 32656

Contact Bob Byrnes

Ph 352.473.2073 Fax 352.473.6501

Private, wholesale. Plants.

Trans Gro Superior Plant

Company Division

510 Frangipani Ave, Naples FL 34117

Contact Neil C Lewi

Ph 239.455.1560 Fax 239.455.2383

E-mail nlewi@gargiulofarms.com

Private, wholesale. Plants.
Transplant Nursery
1586 Parkertown Rd,
Lavonia GA 30553
Contact Jeff Beasley
Ph 706.356.8947 Fax 706.356.8842
E-mail transplants@windstream.net
Private, wholesale, contract. Plants. 


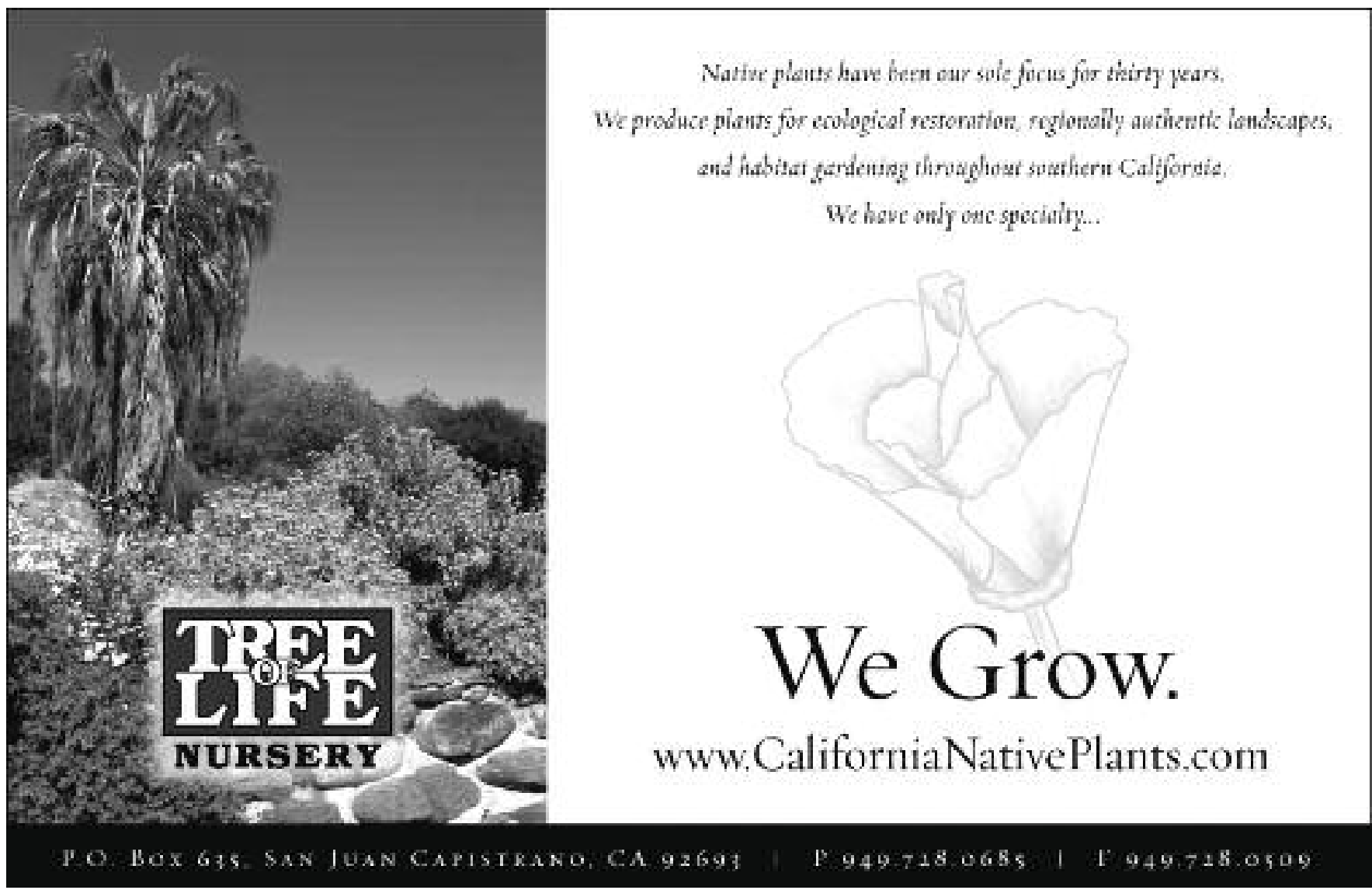

Treasure State Seed Inc PO Box 698, 2380 US Hwy 89, Fairfield MT 59436

Contact Chad Becker

Ph 406.467.2557 Fax 406.467.3377

E-mail treasure@3rivers.net

Private, wholesale, retail. Seeds.

Tree Foundation of Kern

141217 th St, Ste 202,

Bakersfield CA 93301

Contact Melissa Igor

Ph 661.325.6650

E-mail treeinfo@urbanforest.org

Nonprofit, wholesale. Plants.

Tree Frog Farm Inc

3679 Sunrise Rd,

Lummi Island WA 98262

Contact John Robinson

Ph 360.758.7260 Fax 360.758.7260

E-mail info@treefrogfarm.com

Private, wholesale, retail. Plants.
TREE OF LIFE NURSERY

PO Box 635,

San Juan Capistrano CA 92693

33201 Ortega Hwy, San Juan

Capistrano CA 92675

Contact Debbie Evans

Ph 949.728.0685 Fax 949.728.0685

E-mail natives@treeoflifenursery.com

URL

http://www.californianativeplants.com

Private, wholesale, retail, contract, $\mathrm{M}-\mathrm{F}$ $8-4: 30$, since 1978 . Plants: $1,250,000 / y$, $100 \%$ native, $100 \%$ propagated; container, bareroot, cuttings; tree, shrub, herbaceous, grass, wetland/aquatic. Seeds: $100 \%$ native, $100 \%$ propagated; tree, shrub, herbaceous, grass. Min: $\$ 1000$ for contracts; otherwise none.

The finest quality of commercially grown California native plants available. All suitable host plants are inoculated with mycorrhizae.

Tree of Life Nursery is the largest grower of California native plants in the state. Specializing in a line of plants from the southern California region, Tree of Life is dedicated to providing the finest quality of commercially grown plants to the landscaping trade and to the public. Tree of Life Nursery believes in creating spaces that look and feel authentically "Californian."

\section{TREEHAVEN EVERGREEN \\ NURSERY I ARBORETUM \\ ON BLUE HILL}

981 Jamison Rd, Elma NY 14059-9569

Contact Don Hillilker

Ph 716.652 .4206

E-mail conifers@adelphia.net URL http://www.treehavennursery.com Private, wholesale, retail. Plants.

American larch, eastern white pine, Rocky Mountain firs, various pines. Treehaven specializes in the genus Abies (true firs), both native and nonnative. Nonnative types are mostly of China, Japan, and the Mediterranean region. Sales are to Christmas tree growers, landscapers, garden centers, universities, arboretums, and other nurseries and hobbyists. The Arboretum, in a 340-acre preserve in western NY, is a safe haven for many of these firs, being cultured for long-term propagation and source of future plant material. The Arboretum will, in the future, offer additional plant material, such as alderwoods, tamarack, hostas, and rarer true firs. 
TreeMart

12505 N Nebraska, Tampa FL 33612

Contact Mike Lach

Ph 800.664.4006; 813.972.4006

Fax 813.972.4102

E-mail mike@treemart.com

Private, wholesale, retail. Plants.

Trees By Touliatos

2020 Brooks Rd, Memphis TN 38116

Contact Greg

Ph 901.345.7361 Fax 901.398.5217

E-mail treesbytouliatos@bellsouth.net

Private, retail, mail. Plants.

Trees of Corrales

PO Box 1326, 7752 Corrales Rd,

Corrales NM 87048

Contact Desiree Sanchez

Ph 888.418.7337 Fax 505.898.9517

E-mail desiree@treesofcorrales.com

Private, wholesale. Plants.

Trees That Please

3084 Hwy 47, 9 Gilcrease Ln,

Los Lunas NM 87031

Contact Anna Diercks

Ph 505.866.5027 Fax 505.866.SOIL

E-mail treesthatplease@comcast.net

Private, wholesale, retail. Plants.

Triangle Farms

5648 Evans Valley Loop,

Silverton OR 97381

Contact Kevin

Ph 503.873.5190 Fax 503.873.8861

E-mail flowerseed@verizon.net

Private, wholesale, M-F 8-5, since 1991.

Seeds: $5 \%$ native, $75 \%$ propagated.

Triangle Nursery Inc

8526 Beersheba Hwy,

McMinnville TN 37110

Contact Ricky

Ph 931.668.8022 Fax 931.668.3297

E-mail triangle@blomand.net

Private, wholesale. Plants.

\section{TRILLIUM GARDENS}

3362 Videra Dr, Eugene OR 97405

Contact Sheila Klest

Ph 541.937.3073 Fax 541.937.2261

E-mail sheila@trilliumgardens.com

URL http://www.trilliumgardens.com

Private, wholesale, retail, M-F 8-4, since

1986. Plants: $98 \%$ native, $100 \%$ propagated;

Tuolumne Mi-Wuk Native Plants

PO Box 1300, Tuolumne CA 95379

Contact Anna Barajas

Ph 209.928.3793; 512.892.3636

Tribal, wholesale. Plants.
Turner Seed Company Inc 211 Co Rd 151, Breckenridge TX 76424 Contact J Mercer

Ph 254.559.2065 Fax 254.559.5024

E-mail Julie@texasip.com

Private, wholesale, retail. Seeds.

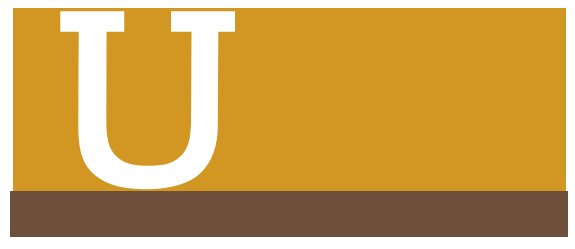

Uncle Tim's Farm \& Garden Center RR 1, Box 205H, Canaan NH 03741 Ph 603.523.4909

Plants.

Underwood Shade Nursery

PO Box 1386,

North Attleboro MA 02763

Ph 508.222.2164 Fax 508.222.5152

E-mailinfo@underwoodshade

nursery.com

Mail, contract. Plants.

University of Florida-Center for

Aquatic and Invasive Plants

7922 NW 71st St, Gainesville FL 32653

Contact William Haller

Ph 352.392.1799 Fax 352.392.3462

E-mailwhaller@ufl.edu

State. Plants.

University of Hawai'i at Manoa-

Harold L Lyon Arboretum

3860 Manoa Rd, Honolulu HI 96822

Ph 808.988.0456 Fax 808.988.0462

State. Plants.

\section{UNIVERSITY OF IDAHO-}

FRANKLIN H PITKIN

FOREST NURSERY

PO Box 441137, University of Idaho, Moscow ID 83844-1137

1025 Plant Science Rd, Moscow ID 83843

Contact Annette Brusven

Ph 208.885.3888 Fax 208.885.6226

E-mail seedlings@uidaho.edu

URL http://seedlings.uidaho.com

State, wholesale, retail, mail, Internet, M-F

$8-4: 30$, since 1910 . Plants: $75 \%$ native,

$100 \%$ propagated; container; shrub.

We grow and sell more than 70 species of tree and shrub seedlings for reforestation, windbreaks, wildlife, restoration, Christmas trees, and more. 


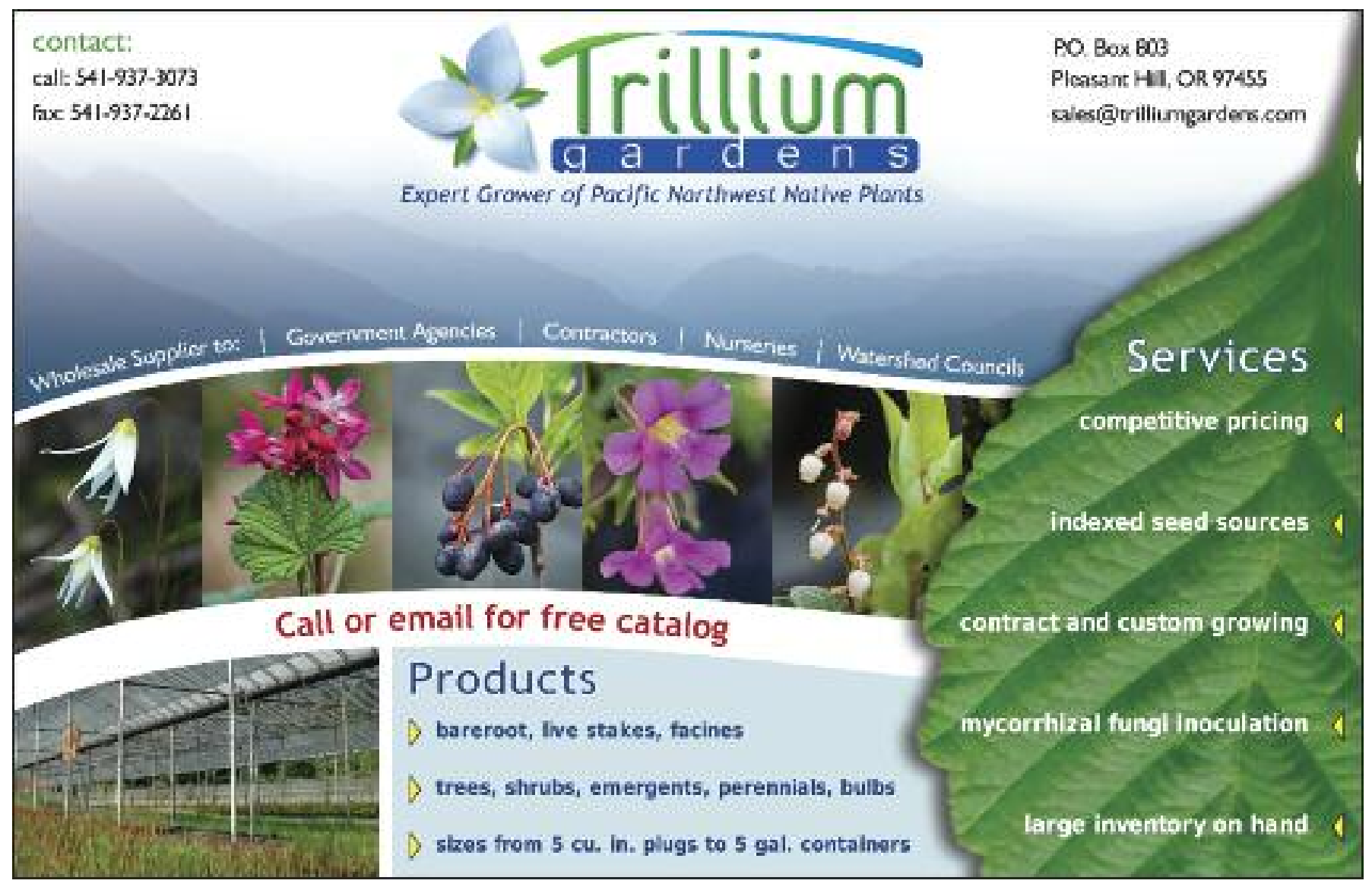

University of Northern lowa-Tallgrass Prairie Center Cedar Falls IA 50614-0294

Contact Gregory Houseal Ph 319.273.3005 Fax 319.268.0668

E-mail gregory.houseal@uni.edu

URL watch for our website coming soon!

Plants, Seeds.

See ad on page 227 .

Urban Forestry Services

301 W Seminary St, Micanopy FL 32667

Contact Michael Campbell

Ph 352.466.3919 Fax 352.466.3280

E-mail urban.forestry.services.forestry@worldnet.att.net

Private, wholesale. Plants, Seeds.

US Fish \& Wildlife Service-Hakalau Forest National

Wildlife Refuge Native Plant Nursery

60 Nowelo St, Ste 100, Hilo HI 96720

Contact Baron Horiuchi

Ph 808.443.2300 ×31

Fax 808.443.2304

E-mail baron_Horiuchi@fws.gov

Federal, no public sales. Plants.

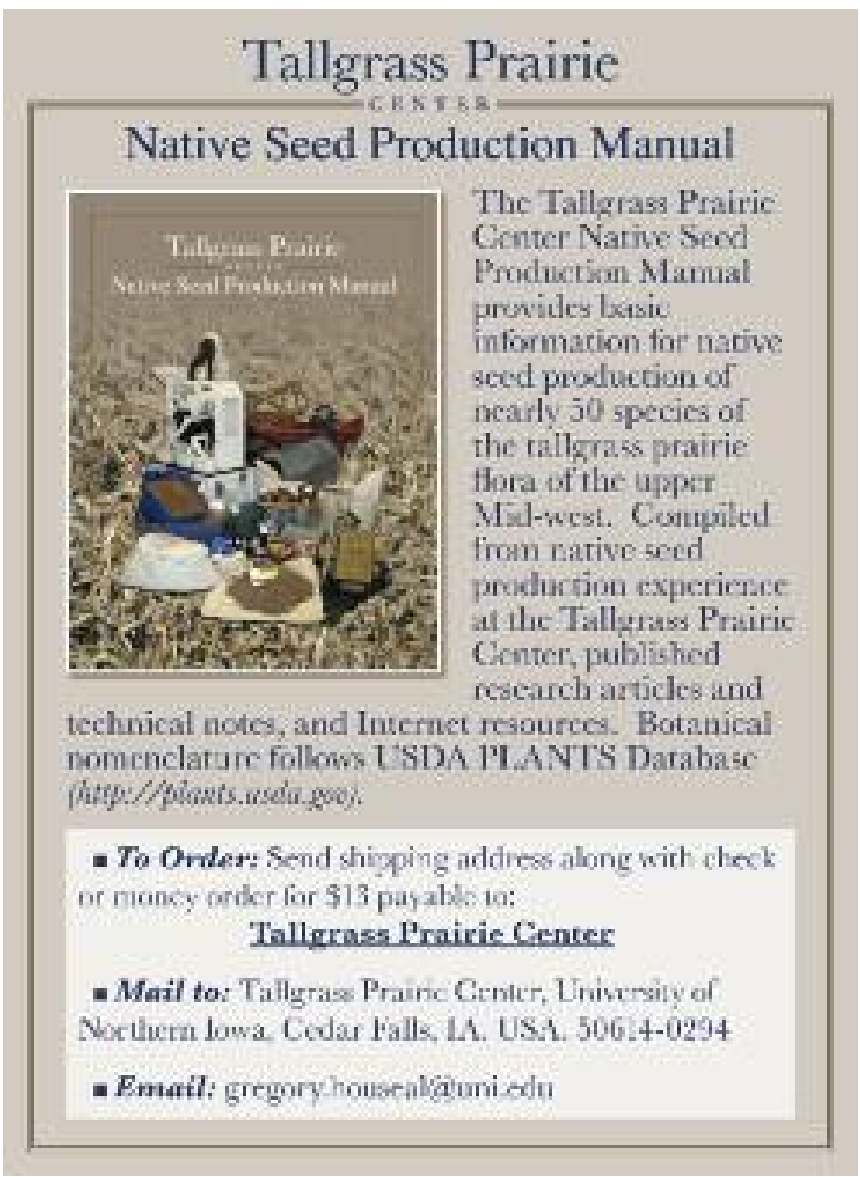


US Fish \& Wildlife ServiceKilauea Lighthouse National Wildlife Refuge Native Plant Nursery

PO Box 1128, Kilauea HI 96754

Contact Ron Langdon and

Shannon Smith

Ph 808.828.1413 Fax 808.828.6634

E-mail shannon_smith@fws.gov

Federal, no public sales. Plants.

USDA Forest Service-Bessey

Nursery

PO Box 39, 40637 River Loop, Hasley

NE 69142

Contact Richard Gilbert

Ph 308.533.2257 x8117; 308.432.0300

Fax 308.533.8116

E-mail regilbert@fs.fed.us

Federal, no public sales. Seeds.

USDA Forest Service-

Coeur d'Alene Nursery

3600 Nursery Rd, Coeur d'Alene ID

83815

Contact Joe Myers or Aram Eramian

Ph 208.765.7375; 208.765.7372

Fax 208.765.7474

Federal, no public sales. Plants.

USDA Forest Service-

Genetic Resource and

Conservation Center

2741 Cramer Ln, Chico CA 95928

Ph 916.895.1176

Federal, no public sales. Plants.

USDA Forest Service-

J Herbert Stone Nursery

2606 Old Stage Rd, Central Point OR

97502

Contact Ken Wearstler

Ph 541.858.6100 Fax 541.858.6110

E-mail kawearstler@fs.fed.us

Federal, no public sales. Plants, Seeds.

USDA Forest Service-

JW Toumey Nursery

PO Box 340, E 23985 Old US 2,

Watersmeet Ml 49969

Contact Tony Holland

Ph 906.358.4523 Fax 906.358 .0493

E-mail aholland@fs.fed.us

Federal, no public sales. Plants, Seeds.
USDA Forest Service-

Lucky Peak Nursery

15169 E Hwy 21, Boise ID 83716

Contact Clark Fleege

Ph 208.343.1977 Fax 208.389.1416

E-mail cfleege@fs.fed.us

Federal, no public sales. Plants, Seeds.

USDA Forest Service-Nebraska

National Forest \& Grasslands

125 N Main, Chadron NE 69337

Federal, no public sales. Plants.

USDA Forest Service-

Ottawa National Forest

E 6248 US 2, Ironwood MI 49938

Federal, no public sales. Plants.

USDA Forest Service-

Placerville Nursery

2375 Fruitridge Rd, Camino CA 95709

Contact Bruce Boom

Ph 530.642.5050; 530.642.506

Fax 530.642.5099

Federal, no public sales. Plants.

USDA Natural Resources

Conservation Service-Rose Lake

Plant Materials Center

7472 Stoll Rd, East Lansing MI 48823

Contact John Leif

Ph 517.641.6300 Fax 517.641.4421

E-mail john.leif@mi.usda.gov

Federal. Plants.

USDA Natural Resources

Conservation Service-Upper

Colorado Environmental Plant

Materials Center

5538 RBC \#4, Meeker CO 81641

Contact Steve Parr, Manager

Ph 970.878.5003 Fax 970.878.5004

E-mail steve.paar@co.nacdnet.net

State. Plants, Seeds.

USDI Bureau of Indian Affairs-

Forest Development

PO Box 189, Mescalero NM 88340

Contact Clay Garrison

Ph 505.464.4410 Fax 505.464.4899

E-mail claygarrison@bia.gov

Federal. Seeds.
USDI Bureau of Indian Affairs-

Jicarilla Agency

PO Box 167, 1 Forestry Dr,

Dulce NM 87528

Contact Steve Thomas

Ph 505.759.3966

E-mail sthomas194@aol.com

Federal. Plants.

USDI Bureau of Indian Affairs-

Red Lake Forestry Greenhouse

PO Box 643, 15757 Mendota Ave,

Redby MN 56670

Contact Gloria Whitefeather-Spears

Ph 218.679.3310 Fax 218.679.2921

E-mail gspears@paulbunyan.net

Tribal, no public sales. Plants.

USDI Bureau of Indian Affairs-

Southern Ute Agency

PO Box 315, 575 Co Rd 517,

Ignacio CO 81137

Contact Ken ZanZee

Ph 970.563.4571 Fax 970.563 .9321

E-mail KenethZanZee@bia.gov

Federal. Plants.

USDI Bureau of Indian Affairs-

Southwestern Indian Polytechnic

Institute

PO Box 10146,

Albuquerque NM 87184

9169 Coors Blvd NW,

Albuquerque NM 87120

Ph 505.766.3170; 800.586.7477

Fax 505.897.5343

Federal. Plants.

USDI Bureau of Land

Management-Charles A Sprague

Tree Seed Orchard

1980 Russell Rd, Merlin OR 97532

Contact Tammy Jebb

Ph 541.476.4432 Fax 541.476.9033

E-mail tjebb@blm.gov

Federal, no public sales. Plants, Seeds.

USDI Bureau of Land

Management-Walter Horning

Seed Orchard

27004 S Sheckly Rd, Colton OR 97017

Contact Michell Finch

Ph 503.630.6080 Fax 503.630.6888

E-mail Michele_Finch@blm.gov

Federal, no public sales. Plants, Seeds. 
USDI National Park ServiceGlacier National Park Native Plant

Nursery

Glacier National Park, West Glacier MT 59936

Contact Sonja Hartmann or Joyce Lapp

Ph 406.888.7835; 406.887.817

E-mail sonja_hartmann@nps.gov

Federal, no public sales. Plants, Seeds.

USDI National Park Service-

Grand Canyon National Park

Native Plant Nursery

PO Box 129,

Grand Canyon AZ 86023-0129

Contact Niki Juarez Cummings

Ph 928.638.7782 Fax 928.638.7755

E-mail niki_juarez.cummings@nps.gov

Federal, no public sales. Plants.

USDI National Park Service-

Haleakula National Park Native

Plant Nurseries

PO Box 369, Makawao HI 96768

Contact Patty Welton

Ph 808.572.4481 x0221

Fax 808.572.4498

E-mail Patti_Welton@nps.gov

Federal, no public sales. Plants.

USDI National Park Service-

Hawai'i Volcanoes National Park

Native Plant Nursery

PO Box 52, Hawaii Volcanoes National

Park HI 96718-0052

Contact David

Ph 808.985.6085 x98 Fax 808.985.6029

Federal, no public sales. Plants.

USDI National Park Service-

Joshua Tree National Monument

for Arid Lands Restoration

74485 National Park Dr,

Twentynine Palms CA 92277

Contact Jean Graham

Ph 760.367.5565 Fax 760.367.6392

E-mail jean_graham@nps.gov

Federal, no public sales. Plants.

USDI National Park Service-

Kalaupapa National Historic Park

Native Plant Nursery

PO Box 2222, Kalaupapa HI 96742

Contact Bill Garnett

Federal, no public sales. Plants.
USDI National Park Service-

Mount Rainier National Park

Native Plant Nursery

Tahoma Woods Star Rt, 55210 238th

Ave E, Ashford WA 98304-9751

Contact Lou Whiteaker

Ph 360.569.2211 x3387

Fax 360.569.2191

E-mail louwhiteaker@nps.gov

Federal, no public sales. Plants.

USDI National Park Service-

North Cascades National Park

Native Plant Nursery

810 SR 20,

Sedro-Woolley WA 98284-1239

Nursery 7280 Ranger Stn Rd,

Marblemount WA 98267

Contact Mike Brondi

Ph 360.854.7275 Fax 360.873.4086

Federal, no public sales. Plants.

USDI National Park ServiceOlympic National Park Native

Plant Nursery

600 E Park Ave,

Port Angeles WA 98362-6798

Contact David Allen

Ph 360.565.3130 Fax 360.565.3147

Federal, no public sales. Plants.

USDI National Park Service-

Rocky Mountain National Park

Native Plant Nursery

1000 Hwy 36,

Estes Park CO 80517-8397

Contact Laura Wheatley

Ph 970.586.1252 Fax 970.586.1392

Federal, no public sales. Plants.

USDI National Park Service-

Sequoia \& Kings Canyon National

Parks Native Plant Nursery

47050 Generals Hwy,

Three Rivers CA 93271-9651

Contact Melanie Baer-Keeley

Ph 559.565.3775; 559.565.3341

Fax 559.565.3730

E-mail Melanie_Baer-Keeley@nps.gov

Federal, no public sales. Plants, Seeds.
USDI National Park ServiceZion National Park Native Plant Nursery

State Rt 9, Springdale UT 84767-1099

Contact Becca Lieberg

Ph 435.772.5662 Fax 435.772.3426

E-mail rebecca_lieberg@nps.gov

Federal, no public sales. Plants, Seeds.

\section{UTAH SEED}

PO Box 16052, Freeport Center Bldg

F9, Clearfield UT 84016

Contact Orson Boyce or Mark Bjarnson

Ph 801.774.0525 Fax 801.774.9711

E-mail oboyce@utahseed.com

Private, wholesale, retail, mail, Internet, M-F

8:30-5, since 2000. Seeds: $2000 / y, 20 \%$

native, $10 \%$ wild collected. Min: $\$ 100$.

We are a full-service seed company providing wildland collected, certified, and sourceidentified seeds. We are located in the heart of the Great Basin and have a network of seed dealers, collectors, and growers. We sell to government agencies and private landowners.

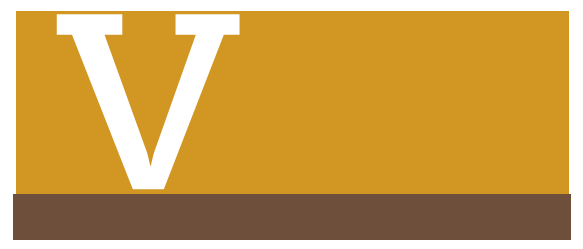

Valdez Native Plants

PO Box 431, 5130 Co Rd 516, Ignacio CO 81137

Contact Johnny

Ph 970.884.0429 Fax 970.884.0429

E-mailvnplants@earthlink.net

Private, wholesale. Plants.

Valley Growers

PO Box 610, 30570 S Barlow Rd,

Hubbard OR 90732

Contact Vicqui Guezara or John D Appel

Ph 503.651.3535 Fax 503.651.3044

E-mailvlygrwrs@web-ster.com

Private, wholesale. Plants.

Valley Nursery

PO Box 4845, 4305 Frontage Dr,

Helena MT 59604

Contact Sharon Teigen

Ph 406.458.3992 Fax 406.458.5919

Private, wholesale, retail. Plants. 
Van Berkum Nursery

4 James Rd, Deerfield NH 03037

Contact Leslie Van Berkum

Ph 603.463.7663 Fax 603.463.7326

E-mail sales@vanberkumnursery.com

Private, wholesale. Plants.

Van Dan Nest Nursery (formerly

Garden of Eden Tree Farm)

9594 Somers Rd, Eden ON NOJ $1 \mathrm{HO}$

Contact Julie Van Dan Nest

Ph 519.866.5269 Fax 519.866.5507

E-mail edentree@mtelecom.net

Private, wholesale. Plants.

Vans Pines Nursery Inc

14731 Baldwin, West Olive MI 49460

Contact Gary Van Slooten

Ph 800.888.7337 Fax 616.399.1652

E-mailinfo@vanspinesnursery.com

Private, wholesale. Plants.

Vienna Nursery

PO Box 306, 980 Rt 45 S,

Vienna IL 62995

Contact George Gellner

Ph 618.658.3621 Fax 618.658.4606

Private, wholesale, retail. Plants.

VIEWCREST NURSERIES

12713 NE 184th St,

Battle Ground WA 98604

Contact Dawna Haluapo

Ph 360.687.5167 Fax 360.687.1212

E-mail bamboo@viewcrest.com

URL http://www.viewcrest.com

Private, wholesale, retail, M-F 8:30-4, since

1954. Plants: $40 \%$ native, $80 \%$ propagated; container, bareroot.

Wholesale container- and field-grown nursery. Collected plants on request.

Vila \& Son Nursery Corporation 20451 SW 216th St, Miami FL 33170 Contact Maria Vila

Ph 305.245.2055 Fax 305.255.9207

E-mailmariav@vila-n-son.com

Private, wholesale. Plants.

Village Nurseries

1589 N Main St, Orange CA 92867

Ph 800.524.0209 Fax 714.279.3199

E-mailvnmailbox@villagenurseries.com

Private, wholesale, retail, contract. Plants.
The Villager Nursery

10678 Donner Pass Rd,

Truckee CA 96161-4834

Contact Sarah Trevilcock

Ph 530.587.0771 Fax 530.587.7439

E-mailinfo@villagernursery.com

Wholesale, retail. Plants.

Vinland Valley Nursery

1606 N 600 Rd, Baldwin City KS 66006

Contact Amy Albright or

Doug Davison

Ph 785.594.2966 Fax 785.594.2787

E-mail amyalbright@vinlandvalley

nursery.com

Private, wholesale, retail, mail. Plants.

Virginia Dept of Forestry-

Augusta Forestry Center

PO Box 160, Crimora VA 24431

Contact Larry

Ph 540.363.7000 Fax 540.363.5055

E-mail afc@dof.virginia.gov

State, wholesale, retail. Plants.

Virginia Dept of Forestry-

Garland Gray Forestry Center

19127 Sandy Hill Rd,

Courtland VA 23837

Contact Dwight

Ph 804.834.2855 Fax 804.834.3141

E-mail dwight.stallard@dof.virginia.gov

State, wholesale. Plants.

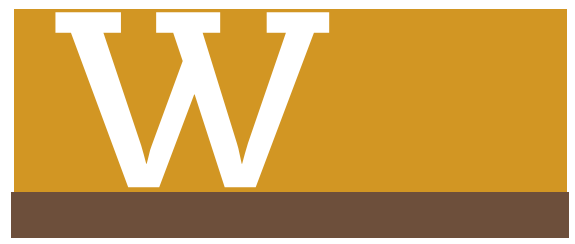

Wabash Farms

PO Box 291, 31218 SE 408th St, Enumclaw WA 98022-0291

Contact Sandy Miller

Ph 360.825.7051 Fax 360.825.1949

E-mail wabash@nventure.com

Private, wholesale, retail, contract, no public sales, by appt, since 1994. Plants:

$200,000 / y, 100 \%$ native, $100 \%$ propagated; container.

We specialize in quality plants from local seed sources. Now raising salt-tolerant tidal plants.

\section{WACD PLANT MATERIALS}

CENTER

16564 Bradley Rd, Bow WA 98232

Contact Jim Brown or Jacquie Gauthier

Ph 360.757.1094 Fax 360.757.3923

E-mail pmcsales@clearwire.net

URL http://www.wacd.org

Private, wholesale, retail, contract. Plants.

The Washington Association of Conservation Districts Plant Materials Center (WACD PMC) is a conservation nursery located in Skagit County, Washington. It grows more than 70 species of native bareroot conservation seedlings, including conifers, shrubs, and deciduous trees. It also grows willows, cottonwoods, and red-osier dogwood live stakes for bioengineering. The WACD has operated this nursery since 1993. Since then, it has worked with conservationists and restorationists to develop a more survivable and vigorous plant. Some of these practices include propagating from source-identified seed, inoculating seedlings with mycorrhizae, and employing different root culturing practices such as root pruning and root wrenching. Contact the PMC to receive a catalog and information about the range of products and services that are offered.

See ad on page 233.

Waimea Valley Arboretum and

Botanical Garden

59864 Kamehameha Hwy,

Hale'iwa HI 96712

Contact David Orr

Ph 808.638.5876 Fax 808.638.7776

E-mail dorr@waimeavalley.net

URL http://www.waimeavalley.net

State, no public sales, M-F 9-5, since 1973.

Plants: $40 \%$ native, $100 \%$ propagated, $30 \%$ wild collected. Seeds.

We exchange plant materials with our conservation partners, but are not a commercial nursery.

A well-labeled collection of more than 5500 types of plants set in a beautiful and historic Hawaiian Valley inland from Waimea Bay on Oahu's north shore.

Walker Nursery Farms

2024 Walt Stephens Rd,

Jonesboro GA 30236

Contact Perry Walker

Ph 770.471.6011 Fax 770.478.1748

Private, wholesale, retail. Plants. 
WALLACE HANSEN NATIVE

\section{PLANTS}

2158 Bower Ct SE, Salem OR 97301

Contact Wallace Hansen

Ph 503.581.2638 Fax 503.581.9957;

503.549.8739

E-mail plants@nwplants.com

URL http://www.nwplants.com

Private, wholesale, retail, mail. Plants.

Warner's Nursery

1101 E Butler Ave, Flagstaff AZ 86001

Contact Misti Warner-Anderson

Ph 928.774.1983 Fax 928.774.6113

Private, wholesale, retail. Plants, Seeds.

WARREN COUNTY NURSERY INC

6492 Beersheba Hwy,

McMinnville TN 37110

Contact Jeff Hobbs

Ph 931.668.8941 Fax 931.668.2245

E-mail wcnursery@blomand.net

URL http://www.tnnursery.com/wcn

Private, wholesale, 7:30-4:30, since 1951.

Plants, Seeds.

Min: $\$ 150 /$ bareroot, $\$ 500 / B \& B / W B$ (unless

picked up).

Warren County Nursery continues to diversify our plant material for our customers. We

always offer you the best product and cus-

tomer service from our family-owned nursery.

See ad on page 233.

WASHINGTON STATE DEPT OF

NATURAL RESOURCES-WEBSTER

FOREST NURSERY

PO Box 47017,

Olympia WA 98504-7017

9805 Blomberg St SW,

Olympia WA 98512

Contact Bill Taylor

Ph 360.753.5305; 877.890.2626

Fax 360.664.0963

E-mail bill.taylor@dnr.wa.gov

URL

http://www.dnr.wa.gov/BusinessPermits

/Topics/WebsterForestNursery/Pages/ab

out_webster_nursery.aspx

State, wholesale, retail, since 1958. Plants:

$100 \%$ native. Seeds.

Water Resources Design Inc

551 Teton Trail, Indianapolis IN 46217

Contact Greg Oskay

Ph 317.786.7529

E-mail goskay25@iquest.net

Private, wholesale, retail. Plants.
Water Wheel Farms

96863 Larson Ln,

North Bend OR 97549

Ph 541.756.6726 Fax 541.756.6726

E-mail konajack@earthlink.net

Private. Plants.

Waterford Gardens

74 E Allendale Rd,

Saddle River NJ 07458

Contact David Meeks

Ph 201.327.0721

Private, wholesale, retail. Plants.

WATERSHED GARDEN WORKS

2039 44th Ave, Longview WA 98632

Contact Scott or Dixie

Ph 360.423.6456 Fax 360.423.6456

E-mail

scott@watershedgardenworks.com

URL http://www.watershedgarden

works.com

Private, wholesale, retail, by appt, since

1989. Plants, Seeds.

A wide variety of native plants propagated primarily from wild seed populations. Wholesale nursery and restoration company offering revegetation and maintenance of mitigation sites.

The Watershed Nursery

601 A Canal Blvd, Richmond CA 94804

Contact Laura Hanson

Ph 510.234.2222 Fax 510.234.2242

E-mail

thewatershednursery@earthlink.net

Private, wholesale, retail. Plants.

Watkins Nurseries Inc

PO Box 160, Powhatan VA 23139

Ph 804.598.6800 Fax 804.598.6220

Private, wholesale. Plants.

Wearren and Sons Nurseries Inc 406 Cotton Ln, Taylorsville KY 40071 Contact Brent Wearren

Ph 502.252.7788 Fax 502.252.7349

E-mail wearrenson@aol.com

Private, wholesale, retail. Plants.

Webb Landscape Inc

162 Glendale Rd, Bellevue ID 83313

Contact Mark Palmer

Ph 208.788.2066 Fax 208.726.4767

E-mail markpalmer@webbland.com

Private, retail. Plants.
West Kootenay Plants

3279 Little Slocan S Rd,

Winlaw BC V0G 2J0

Contact $\mathrm{E}$ Johansson

Ph 250.226.7309 Fax 250.226.7310

E-mailinfo@wkp.ca

Private, wholesale, contract, since 2005.

Plants: $60,000 / y, 100 \%$ native, $100 \%$ propa-

gated, $10 \%$ wild collected; container.

Native plants for restoration, landscaping, and agro-forestry. Container-grown from sustainably collected West Kootenay seed.

West Virginia Division of Forestry-Clements State Tree Nursery

PO Box 8, 624 Forestry Dr, West Columbia WV 25287

Contact David K McCurdy or

Jason Huffman

Ph 304.675.1820

E-mail clements@wvnet.edu

State, $8-5$. Plants: $50 \%$ native, $100 \%$ propagated, $20 \%$ wild collected.

Westchester Community

College-Native Plants Center

75 Grasslands Rd, Valhalla NY 10595

Contact Brooke Beebe

Ph 914.606.7870 Fax 914.606.6143

E-mail wcc.nativeplant@sunywcc.edu

Private, retail, M-F 9-5, since 2000. Services.

Public education, conferences, lectures, affiliate of Ladybird Johnson Wildflower, native plant advice for government agencies; not a supplier.

Western Forest Systems Inc 3731 15th St, Lewiston ID 83501 Contact Jan Schaefer

Ph 208.743.0147

E-mail schaeferjk@clearwire.net

Private, wholesale, retail, M-F 7-3, since

1985. Plants: 3,000,000/y, 99\% native,

$100 \%$ propagated.

Reforestation consulting and contract growing.

Western Maine Nurseries

PO Box 250, 4 Nursery Ln, Fryeburg

ME 04037

Contact Robin Libby

Ph 800.447.4745; 207.935.2161

Fax 207.935.2043

E-mailinfo@westernmainenurseries.com

Private, wholesale. Plants. 
Western Native Seed

PO Box 188, Coaldale CO 81222

Contact Alex Tonnesen

Ph 719.942.3935 Fax 719.942.3605

E-mailinfo@westernnativeseed.com

Private, wholesale, retail, mail. Plants, Seeds.

Westlake Nursery at Farout Farm 05720 Canary Rd, Westlake OR 97493

Contact John Evanow

Ph 541.997.6071

E-mail johnevono@oregonfast.net

Private, wholesale. Plants.

Westland Seed Inc

36272 Round Butte Rd,

Ronan MT 59864

Contact Bill Bartell

Ph 800.547.3335; 406.644.2202

Fax 406.676.4101

E-mail westland@ronan.net

Private, wholesale, retail. Seeds.

Weston Gardens in Bloom Inc 8101 Anglin Dr, Fort Worth TX 76140

Contact Weston

Ph 817.572.0549 Fax 817.478.7220

E-mail weston@westongardens.com

Private, retail. Plants, Seeds.

Westscape Wholesale Nursery 423 N Tracy, Bozeman MT 59715

110 Progressive Dr, Belgrade MT 59174

Contact Laura Smith or Robert Dunn

Ph 406.388.1116 Fax 406.388.1116

E-mail westscapenursery@yahoo.com

Private, wholesale. Plants.

Wetland Supply Company I

Native Plant Nursery

194 Goodview Dr, Apollo PA 15613

Contact Dave Hails

Ph 724.727.3772 Fax 724.727.3778

E-mailwetplants@aol.com

Private, wholesale. Plants, Seeds.

Wetlands \& Woodlands

Wholesale Nursery

12800 35th Ave SE, Everett WA 98208

Contact Al

Ph 425.338.9218 Fax 425.337.4985

E-mail ellasmith@wetlandsandwood

lands.com

Private, wholesale. Plants.
Wetlands Nursery

PO Box 14553, Saginaw MI 48601

Contact Jewel Richardson

Ph 989.752.3492 Fax 989.752.3096

E-mail jewel.richardson@att.net

Private, wholesale, retail. Plants.

Weyerhaeuser Company-

Aurora Forest Nursery

$6051 \mathrm{~S}$ Lone Elder Rd,

Aurora OR 97002

Contact Mark Triebwasser

Ph 503.266.2018 Fax 503.266.2010

E-mail mark.triebwasser@weyer

haeuser.com

Forest Industry, wholesale, retail, contract.

Plants.

Weyerhaeuser Company-

Magnolia Nursery

2960 Columbia $11 \mathrm{E}$,

Magnolia AR 71753

Contact Kevin Richardson

Ph $870.234 .3537 \times 14$

Fax 501.234.7918

Forest Industry, retail. Plants.

Weyerhaeuser Company-

Quail Ridge Nursery

169 Weyerhaeuser Rd, Aiken SC 29801

Contact Bruce Francis

Ph 800.634.8975 x1 Fax 803.649.0997

Forest Industry. Plants.

Weyerhaeuser Company-

Rochester Greenhouse

7935 Hwy 12 SW,

Rochester WA 98579-9214

Contact Mike Pfaff

Ph 360.273.5527 Fax 360.273.6048

E-mail mike.pfaff@weyerhaeuser.com

Forest Industry, wholesale. Plants.

Weyerhaeuser Company-

Turner Nursery

16014 Pletzer Rd SE, Turner OR 97392

Contact Sue Woodall

Ph 800.732.4769 Fax 541.327.2591

E-mail sue.woodall@weyerhaeuser.com

Forest Industry, retail, contract. Plants.

Weyerhaeuser Nursery

3890 Hwy 28 W, Camden AL 36726

Contact Kimmie Van Wyck

Ph 800.635.0162 ×24

Fax 251.634.4481
E-mail ralph.bower@weyerhaeuser.com Forest Industry. Plants.

Wheatley Woods Native Plant

Nursery and

Garden Centre

Box 765, 2448 Essex Rd 14, Wheatley

ON NOP 2PO

Contact Craig Willett

Ph 519.825.4217 Fax 519.825.7239

E-mail sales@wheatley-woods.com

Private, wholesale, retail. Seeds.

Whisper Palms of Terra Ceia LLC

4400 118th Ave N, Ste 305, Clearwater

FL 33762

Ph 941.722.8383 Fax 727.572.1292

E-mail whisper01@sprynet.com

Wholesale. Plants.

White City Nursery LLC

707 Co Rd 20 W, Verbena AL 36091

Contact Louis Olivier

Ph 334.365.2488 Fax 334.365.2488

Private, wholesale. Plants.

White Flower Farms

PO Box 50, Litchfield CT 06759

167 Litchfield Rd, Morris CT 06763

Ph 800.503.9624; 860.482.3638

Fax 860.496.1418

E-mail custserv@whiteflowerfarm.com.

Private, wholesale, retail. Plants.

Whitman Farms

3995 Gibson Rd NW, Salem OR 97304

Contact Lucile Whitman

Ph 503.585.8728 Fax 503.363.5020

E-mail lucile@whitmanfarms.com

Private, wholesale, retail, mail, 7-5, since

1980. Plants: $20 \%$ native, $99 \%$ propagated.

Wichita Nursery Inc

9413 S Hienz Rd, Canby OR 97013

Contact Jim

Ph 503.651.2279 Fax 503.651.3732

E-mail jim@wichitanursery.com

Private, wholesale. Plants.

Wichita Valley Nursery

5314 Southwest Parkway,

Wichita Falls TX 76310

Ph 940.696 .3082

E-mailwvmail@aol.com

Private, retail. Plants. 
Wicklein's Water Gardens

PO Box 42080, Baltimore MD 21286

1820 Cromwell Bridge Rd,

Baltimore MD 21234

Contact Erik Wicklein

Ph $410.823 .1335 \times 11$

Fax 410.823.1427

E-mail ewicklein@earthlink.net

Private, wholesale. Plants.

Wilcox Nursery

12501 Indian Rocks Rd,

Largo FL 33774-3037

Contact Bruce Turley

Ph 727.595.2073 Fax 727.595.6963

E-mail wnursery@tampabay.rr.com

Private, wholesale, retail. Plants.

\section{WILD Canada}

75 39th St N,

Wasaga Beach ON L9Z 2A3

Contact Scott Martin

Ph 705.429.4936 Fax 705.429.1435

E-mailinfo@wildcanada.ca

Private, wholesale, mail, M-F 9-5; by appt, since 1989. Plants.

WILD Canada produces 250 species of native plants for central and southern Ontario for use in habitat creation/restoration and natural landscaping projects.

Wild Ginger Native Plant Nursery

6752 Perrytown Rd,

Port Hope ON L1A 3V5

Ph 705.740.2276

Plants.

Wild Rose Ranch

50665 NW Staley Rd, Banks OR 97106

Contact Dawn

Ph 503.324.5580 Fax 503.324.3094

E-mail wildrranch@aol.com

Private, wholesale. Plants.

\section{Wild Seed}

PO Box 27751, Tempe AZ 85285

Ph 602.276.3536 Fax 602.276.3524

Wholesale, retail, mail. Seeds.

\section{Wildflower}

234 Oak Tree Trail,

Wilsonville AL 35186

Contact Jan Midgley

Ph 205.602.2563

E-mail jwildflwr@aol.com

Private, wholesale, retail. Plants.

\section{Native Trees \& Shrubs}

- Quality Native Trees \& Shrubs •

- Contract Growing •

- Custom Propagation -

- Willow \& Cottonwood Live Stakes •

\section{Providing the conservation and restoration community with conservation grade seedlings and cuttings since 1993.}

Jacquic Gauthier, Sales Manager James Brown, Manager

WACD Plants Materials Center 16564 Bradley Road

Bow WA 98232

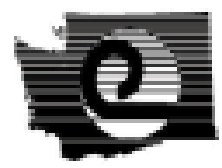

360-757-1094

WACD Plant Materials Center

pmcsales@clearwire.net

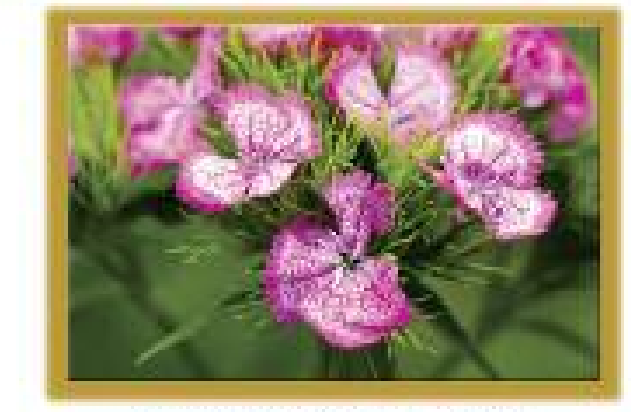

Native Plants \& Seeds

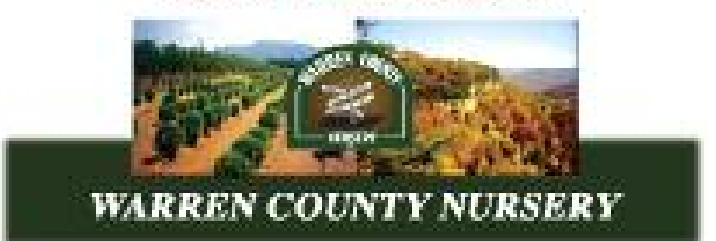

Sicce 7951

Visit ous nursery on the web! Shade \& Oruamertal I Fruit. Shade, \& Nut Trees Btoadleaf \& Conifer Evesereens f Ground Covers Flowering Shruks I Fema I Wildfiowers

\section{wwhtmnarserycom/wen}

6492 Beersheba Ifwy McMianvilie TN $37 J 0$

931.668.894i Jeff Hobhs: mceursery 9 blomasd.ne! 
Wildflower Farm Inc

10195 Hwy 12 W RR \#2,

Coldwater ON LOK 1E0

Contact Paul Jenkins or Miriam

Ph 866.476.9453

E-mail info@wildflowerfarm.com

Private, wholesale, retail. Plants, Seeds.

Wildflower Nursery

4920 NE 25-70, Marshall NC 28753

Contact Dewey or Maggie Griffey

Ph 828.656.2723 Fax 828.656.2723

E-mailinfo@wildflowernursery.net

Private, wholesale, retail, mail. Plants.

The Wildflower Seed Company

PO Box 406, St Helena CA 94574

Ph 707.963.3359 Fax 707.963.5383

E-mailsales@wildflower-seed.com

Private, mail. Seeds.

Wildland Nursery

370 E 600 N, Joseph UT 84739

Contact Janett Warner

Ph 435.527.1234; 435.527.5020

Fax 435.527.1234

E-mail janett@wildlandnursery.com

Private, wholesale, retail. Plants.

WILDLANDS NURSERY

1941 Saint St, Richland WA 99354

68911 N River Rd,

Benton City WA 99320

Contact Kirk Anderson

Ph 509.588.4328 Fax 509.588.2759

E-mailinfo@wildlandsnursery.com

URL www.wildlandsnursery.com

Private, wholesale, retail, contract, M-F; 8-4:30; by appointment, since 2000. Plants: $100,000 / y, 95 \%$ native, $95 \%$ propagated, $5 \%$ wild collected; container, bareroot, cuttings, bioengineering; tree, shrub, herbaceous, grass.

Wholesale grower providing more than 170 species of hardy plants from the Intermountain West and Pacific Northwest. We provide plants that are locally adapted and source-identified, ranging in size from plugs to 20 gallon containers. Custom seed/cutting collection and contract growing available. We provide our own climate controlled delivery for any sized order to ensure that the highest quality plant material arrives at your project site. Wildlands Nursery is owned by Wildlands Inc, which is a full service restoration company serving the western US since 1987. Wildlands Inc, restoration services include: project design and planning, consulting, installation, erosion control, seeding, bioengineering, weed control, and monitoring.
WILDLIFE HABITAT NURSERY

1025 E Hatter Creek Rd,

Princeton ID 83857

Contact Denny Dawes

Ph 208.875.2500 Fax 208.875.8900

E-mailwild@potlatch.com

URL www.whn-online.com

Private, wholesale, retail, M-Sat 7-3:30, since 1992. Plants: $250,000 / y, 99 \%$ native, $100 \%$ propagated; container, cuttings, bioengineering; tree, shrub, herbaceous, grass.

Supplier of native trees, shrubs, herbaceous plants for wildlife habitat, restoration, and erosion control; specializing in growing container seedlings for wetlands and riparian habitats. Our willow poles are especially effective. Also sale of planting supplies, tree tubes, and repellents.

Wildlife Nurseries Inc

PO Box 2724, Oshkosh WI 54903

Contact Jim or Sue Lemberger

Ph 920.231.3780 Fax 920.231.3554

Private, wholesale, retail, mail. Plants, Seeds.

Wildseed Farms

PO Box 3000, 425 Wildflower Hills,

Fredericksburg TX 78624

Contact Gary Grasshof

Ph 800.848.0078

E-mail gary@wildseedfarms.com

Private, wholesale, retail. Seeds.

Wildside Growers

6360 Hannegan Rd, Lynden WA 98264

Contact Veronica or Sue

Ph 360.398.7158 Fax 360.398.7158

E-mail wildsidegrowers@comcast.com

Private, wholesale. Plants.

Wildtype Design Native Plants

$\&$ Seed Ltd

900 N Every Rd, Mason Ml 48854

Contact Bill Schneider

Ph 517.244.1140 Fax 517.244.1142

E-mail wildtype@msu.edu

Private, wholesale, retail, contract. Plants, Seeds.

Wildwood Farm

10300 Sonoma Hwy,

Kenwood CA 95452

Contact Sara Monte

Ph 707.833.1161

Private, retail, mail. Plants.
Willamette Gardens

3290 SW Willamette Ave,

Corvallis OR 97333

Contact Esther Gruber McEvoy

Ph 541.754.0893 Fax 541.754.9326

E-mail natives@willamettegardens.com

Private, wholesale, retail, contract. Plants.

Wilson Greenery

PO Box 2554, Shelby NC 28151

1310 Polkville Rd, Shelby NC 28150

Contact Beverly

Ph 704.484.2709 Fax 704.482.6870

Private, wholesale. Plants.

Wilson Seed Farms

108721400 East St, Tiskilwa IL 61368

Contact Chris Wilson

Ph 815.878.8572 Fax 815.366.9050

E-mail cwilson5@hotmail.com

Wholesale, retail. Seeds.

\section{WIND RIVER SEED}

3075 Ln 51-1/2, Manderson WY 82432

Contact Russ Holzhauser

Ph 307.568.3361

E-mail russ@windriverseed.com

URL http://www.windriverseed.com Private, wholesale, retail, M-F 8-5, since 1973. Seeds: $100 \%$ native, $90 \%$ propagated. Min: $\$ 50$.

Wild collect and grow seed sources. We provide seed for projects in the Intermountain West and northern Great Plains, including grasses, forbs, wildflowers, legumes, wetlands, forage, range, and turf seed, including droughttolerant turf alternatives. Our competent staff provides ph consultation to help design the mix to suit your site. Questions about seed? Ask Wind River Seed.

Windy Hill Plant Farm

40413 John Mosby Hwy,

Aldie VA 20105

Contact Karen

Ph 703.327.4211 Fax 703.327.4221

E-mailwindyhill@windyhill.net

Private, retail. Plants.

Windy Hills Farm

1565 E Wilson Rd, Scottville MI 49454

Contact Norman Letsinger

Ph 231.757.2373

E-mailwindyhills@gtlakes.com

Private, wholesale, mail. Seeds. 
Windy Ridge Tree Farm \& Wholesale Nursery

PO Box 340, Amboy WA 98601

Contact Robert Kramer

Ph 360.247.TREE

E-mail fastgrowingtrees@yahoo.com

Private, wholesale. Plants.

Winter Garden Nursery

6840 FM 1436, La Pryor TX 78872

Ph 830.279.2759

E-mail info@wintergardennursery.com

Private, wholesale. Plants.

WinterCreek Restoration

PO Box 1543, Bend OR 97709

63405 Deschutes Market Rd,

Bend OR 97701

Contact Karen Theodore

Ph 541.948.0063; 541.382.1227

Fax 541.382.1227

E-mail karen@wintercreeknursery.com

Private, wholesale, M-F 8-4 weekends by appt, since 1993. Plants:

$300,000 / y, 100 \%$ native, $50 \%$ propagated, $10 \%$ wild collected; con-

tainer, $B \& B$, cuttings.

Dryland natives of the high desert and Great Basin.

WINTERHAVEN WILDFLOWERS \&

NATIVE PLANT PRESERVE

5724 S 900 W, West Point IN 47992

Contact Reni Winter

Ph 765.714.4288

E-mail reni@winterhavenfarm.us

URL http://www.winterhavenfarm.us

Private, wholesale, retail. Plants.

A 13-acre alfalfa farm being restored into a tall-grass prairie and native plant nursery and preserve. The wholesale and retail nursery helps support and sustain the on-site preservation and education efforts of Reni Winter, owner, grower, and native plant preservationist. Tours, nature encounters, workshops, and dig-your-own, as well as potted plants are available.

See ad on page 235 .

Wisconsin Dept of Natural Resources-

Griffith State Forest Nursery

473 Griffith Ave,

Wisconsin Rapids WI 54494

Ph 715.424.3700 Fax 715.421.7830

State. Plants.

Wisconsin Dept of Natural Resources-Hayward

State Forest Nursery

16133 W Nursery Rd,

Hayward WI 54843

Contact Gordon or Greg Edge

Ph 715.634.2717 Fax 715.634.7642

State. Plants, Seeds.

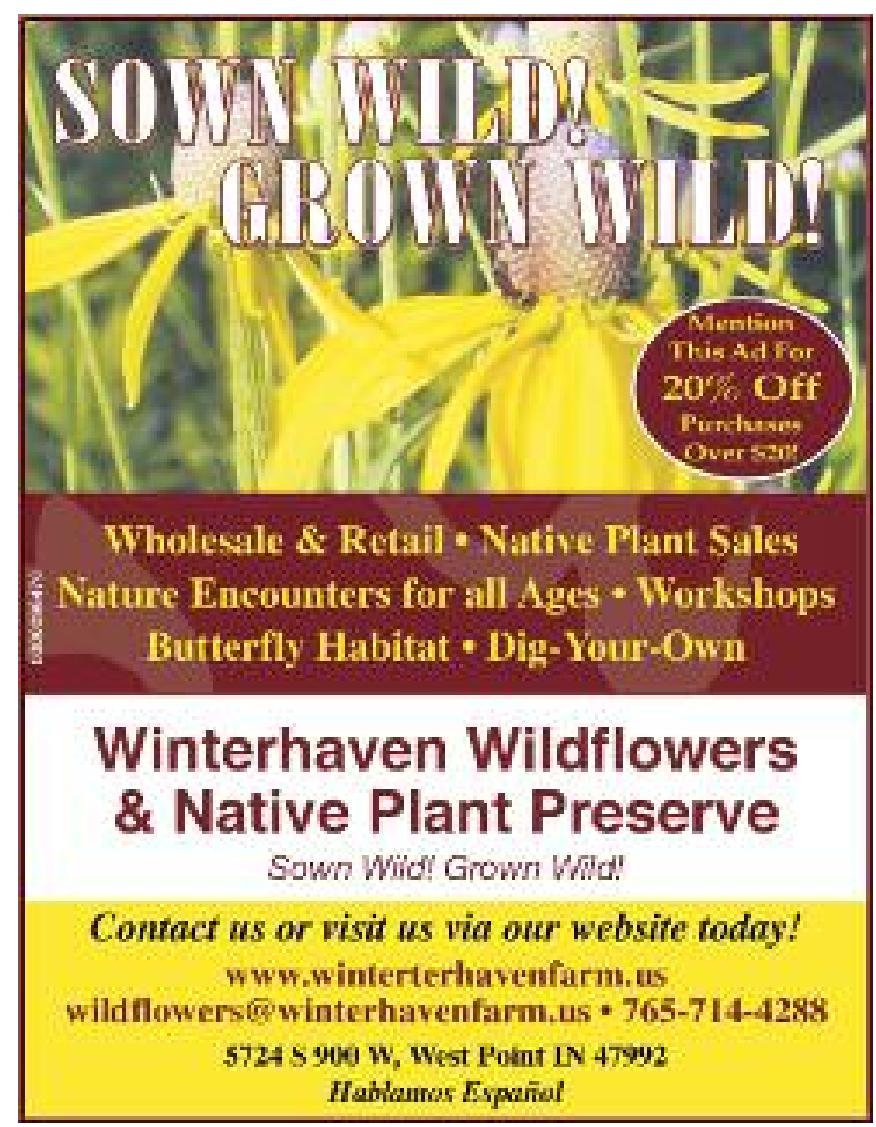

Wisconsin Dept of Natural Resources-

Wilson State Forest Nursery

PO Box 305, 5350 Hwy 133 E, Boscobel WI 54805

Contact Joe Vandehey

Ph 608.375.4123 Fax 608.375.4126

E-mail joe.vandehey@dnr.state.wi.us

State, retail, M-F 7:45-4:30, since 1952. Plants: 5,000,000/y, 100\% native, $100 \%$ propagated, $80 \%$ wild collected. Seeds.

Seedlings sold for reforestation and conservation purposes. Seedling may not be resold or used for ornamental or landscaping.

Wolfater Native Seeds

Box 177, Eastend SK SON 0T0

Contact Robin

Ph 306.558.4700 Fax 306.558.4920

Private, retail. Seeds.

Womack Nursery Company

2551 SH 6, De Leon TX 76444-6333

Contact Larry J Womack or

Larry Don Womack

Ph 254.893.6497 Fax 254.893.3400

Private, retail, mail. Plants. 
Wood's Edge Farm

532 Stanek Rd,

Muscoda WI 53573-9448

Contact Martha Peterson

Ph 608.739.3527 Fax 608.739.3527

E-mailinfo@woodsedgefarm.com or woodedge@mwt.net

Private, wholesale, retail, mail, M-F 9-5, since 1994. Plants: $2000 / y, 100 \%$ native,

$100 \%$ propagated; container, bareroot.

Nursery-propagated wildflowers native to the woodlands and savannas of the upper Midwest.

\section{WOODBROOK NURSERY}

5919 78th Ave NW,

Gig Harbor WA 98335

Contact Ingrid Wachtler

Ph 253.265.6271; 253.225.1900

Fax 253.265.6471

\section{E-mail woodbrk@harbornet.com}

URL http://www.woodbrooknativeplant nursery.net

Private, wholesale, retail, contract, T-Sat 10-4, since 1994. Plants: 30,747/y, 99\% native, $99 \%$ propagated, $1 \%$ wild collected; container, $B \& B$, cuttings; tree, shrub, herbaceous, grass, wetland/aquatic.

Come visit us for a pleasant trip to scenic Gig Harbor, Wash.! We specialize in growing more than 120 types of Pacific Northwest Native Plants. Woodbrook Nursery is one of the largest retail outlets for native plants in the greater Seattle, Tacoma, and Olympia area with a native-knowledgeable sales staff. Let us help with your ornamental native garden, creating wildlife habitat in your yard, or wetland mitigations. We also contract grow and/or sell to government agencies and contractors and other wholesale customers. We now have more retail-friendly hours, open Tuesdays through Saturdays, 10 am to $4 \mathrm{pm}$, except some holidays (check our website).

See ad on page 237.

\section{WOODLANDERS INC}

1128 Colleton Ave, Aiken SC 29801

Contact Robert McCartney

Ph 803.648.7522 Fax 803.648.7522

E-mailinfo@woodlanders.net

URL http://www.woodlanders.net/

Private, mail, Internet, M-F 9-5, since 1980.

Plants: $60 \%$ native, $100 \%$ propagated.

Pioneer in native plant propagation and introduction. Hundreds of species and selections first offered by Woodlanders Inc now in gardens worldwide. Our stock is native to North America. Our emphasis is on rare and hard-tofind plants. Shipping October-March.
Woodmere Nursery Ltd PO Box 498 Hwy 2, 100th Ave (Range Rd 32), Fairview AB TOH 1LO

Contact Jeff Hoyem

Ph 780.835.5292 Fax 780.835.5459

E-mail

Woodmere.Nursery@wispernet.ca Private, wholesale. Plants.

Woodsman's Native Plants 4385 Hwy 101 N, Florence OR 97439 Contact Dale or Lisa Ph 541.997.2252 Fax 541.997.1960

Private, wholesale, retail. Plants.

Woody Warehouse Nursery PO Box 259, 3216 W Co Rd 850 N, Lizton IN 46149

Contact Pete Berg

Ph 317.994.5497; 866.766.8367

Fax 317.994.5494

E-mail sales@woodywarehouse.com Private, wholesale, retail, contract. Plants.

Wrights Nursery

6040 FM 2657, Briggs TX 78608

Ph 512.489.2239 Fax 512.489.2837

E-mail sales@wrightsnursery.net Private, wholesale, retail. Plants.

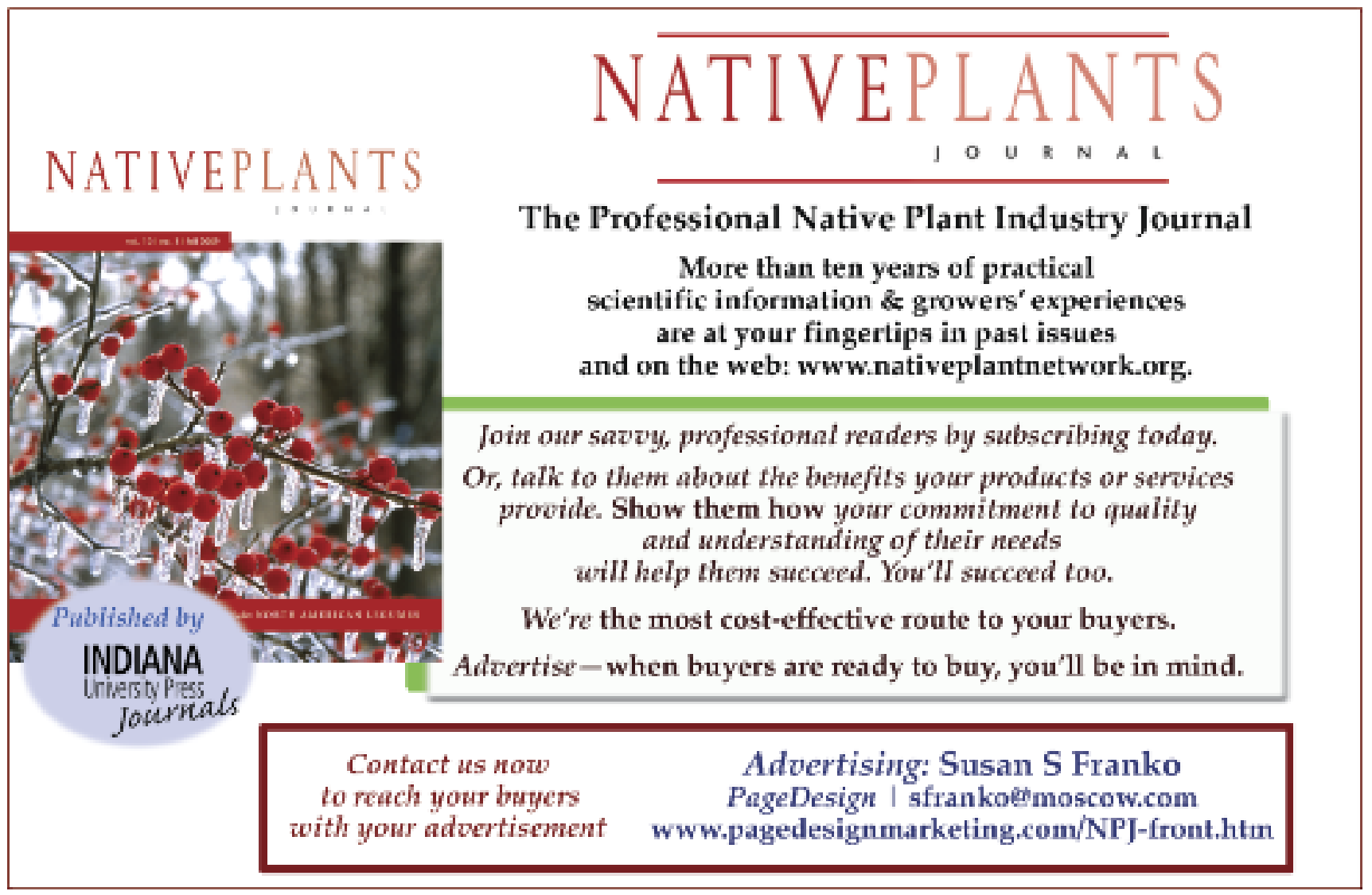




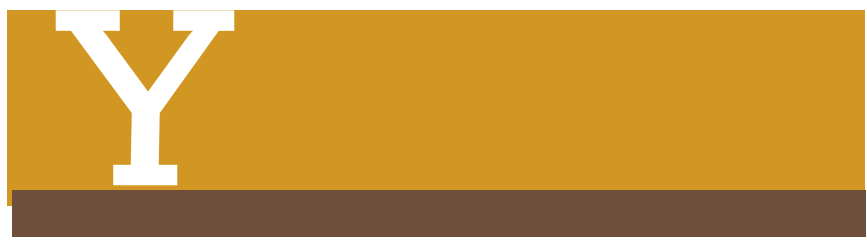

Yankee Gardener Greenhouses and Nursery 132 Smutty Hollow Rd, Monroe NH 03771

Contact Terry Ward Ph 603.638.2809 Fax 603.638.2809

Private, wholesale, mail. Plants.

Yellow Mountain Farms

PO Box 39 or 38, Minneapolis NC 28652

Ph 828.733.5705 E-mail arthrugriffith@mac.com

Plants.

YELLOW SPRINGS FARM

1165 Yellow Springs Rd, Chester Springs PA 19425

Contact Catherine Renzi

Ph 610.827.2014 Fax 610.482.9311

E-mail catherine@yellowspringsfarm.com

URL http://www.yellowspringsfarm.com

Private, wholesale, retail, year-round, since 2002. Plants: $98 \%$ native, $100 \%$ propagated.

Specializing in perennials, grasses, shrubs, and trees native to PA and the Mid-Atlantic area. Our former dairy farm dates to 1851. Interests in land stewardship, education, native plants for restoring and maintaining a diverse ecosystem. Visitors welcome by appointment.

Yellowpoint Propagation Ltd

13735 Quennell Rd, Box 669, Ladysmith BC V9G 1 A5

Contact Don Pigott Ph 250.245.4635 Fax 250.245.5935

E-mail ypprop@shaw.ca

Private, wholesale. Seeds.

Yerba Buena Nursery

19500 Skyline Blvd, Woodside CA 94062

Contact Kathy Crane Ph 650.851.1668 Fax 650.851.5565

Private, retail. Plants.

\section{YUCCA DO NURSERY}

PO Box 1039, Giddings TX 78942-1039

Ph 979.542.8811 E-mail info@yuccado.com

URL http://www.yuccado.com

Private, retail, mail, Internet. Plants.

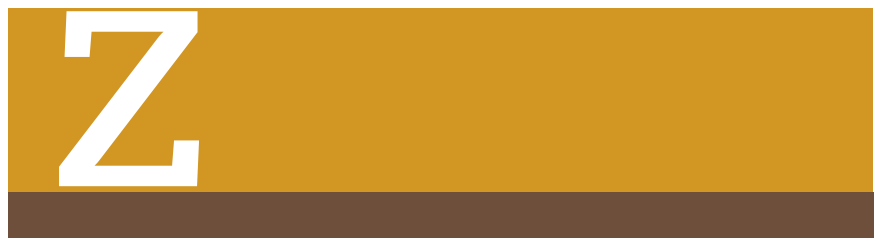

Zelenka Evergreen Nursery

Liner/Seedling Production, 16127 Winans, Grand Haven Ml 49417

Contact Todd Bustard Ph 616.842.1367 Fax 616.842.0304

E-mail info@zelenkanursery.com

Private, wholesale. Plants.

\section{Woodbrook Native Plant Nursery}

\section{Growing Pacific Northwest Native Plants}

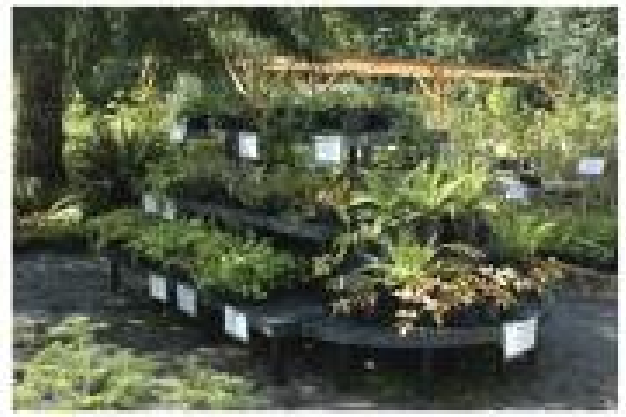

Wholesale

Retail

Contract

Growing

Farm Location: $591978^{\circ}$ Ave NW, Gig Harbor, WA 98335

Ph: 253-265-6271; Fax: 253-265-6471; Cell: 253-225-1900

Hours: Tue,-Sat. 10 AM to 4 PM (except some holidnys); Wholesale eustomers need appointment.

Website: www, woodhrooknativeplantnursery.com

Email: woodbrkat harbornetcom 


\section{ALABAMA}

Plants

Alabama Forestry Commission-EA Hauss Nursery

ArborGen-Alabama SuperTree Nursery

DeepSouth Pine Nursery Inc

Dodd \& Dodd Native Nurseries

International Paper Company-Union Springs

SuperTree Nursery

Joshua Timberlands Nursery

Molpus Timberlands Management LLC I Joshua

Timberlands Nursery

Old South Nurseries

RE Mitchell Nursery

Sand Mountain Herbs

TOM DODD NURSERY

Weyerhaeuser Nursery

White City Nursery LLC

Wildflower

Seeds

Molpus Timberlands Management LLC I Joshua Timberlands Nursery

Sand Mountain Herbs

\section{ALASKA}

Plants

AgriCycle Farms

Alaska Dept of Natural Resources_-Plant Materials

Center Forest Nursery

Alaska Division of Forestry

Alaska Greenhouses Inc

Baker's Acres

Fritz Creek Gardens

Holm Town Nursery Inc

Kenaitze Greenhouse and Gardens

Landscape Alaska

Seeds of Alaska
Seeds

Alaska Dept of Natural Resources-

Plant Materials Center Forest Nursery

Far North Tree and Seed Company

Landscape Alaska

Seeds of Alaska

\section{ALBERTA}

Plants

Alberta Nurseries and Seeds Ltd

ALCLA Native Plant Restoration Inc

Bow Point Nursery Ltd

Brett Young

Eagle Lake Nurseries Ltd

K\&C Silviculture Farms Ltd

Native Plant Restoration Inc

Woodmere Nursery Ltd

\section{Seeds}

Alberta Nurseries and Seeds Ltd

Native Plant Restoration Inc

Prairie Seeds Inc

\section{AMERICAN SAMOA \\ Plants}

American Samoa Community CollegeLand Grant Forestry Extension

\section{ARIZONA}

Plants

'Ahakhav Tribal Preserve

Arid Solution LLC

Arid Zone Trees

Arizona Botanical

Arizona Native Plant Specialists I A Great

Southwest Cactus LLC

Arizona's Best Native Plant Care Inc

Bach's Cactus Nursery Inc

Biddles Nursery
Desert Enterprises

Desert Survivors

Desert Trees Nursery

Flagstaff Native Plant and Seed

Flagstaff Native Plant Nursery

Fort Apache Agency-White Mountain Apache

Kelly Green Trees Inc

Mesquite Valley Growers

MOUNTAIN STATES

Native Sun Home \& Garden Nursery

Navajo Forestry Nursery

Nighthawk Natives Nursery \& Wildlands

Restoration

Oaks of the Wild West

Plants for the Southwest I Living Stones Nursery

Rascon Landscaping and Nursery

Sinagua Nursery

Spadefoot Nursery

USDI Bureau of Indian Affairs-McNary

Greenhouse

USDI National Park Service-Grand Canyon

National Park Native Plant Nursery

Warner's Nursery

Seeds

Flagstaff Native Plant and Seed

Mohave Joshua Company

Native Seeds

Nighthawk Natives Nursery \& Wildlands Restoration

Rascon Landscaping and Nursery

SEEDS TRUST I HIGH ALTITUDE GARDENS

Southwestern Native Seeds

Warner's Nursery

Wild Seed 


\title{
location index
}

\author{
NATIVE PLANT MATERIAL SUPPLIERS BY STATE, PROVINCE, OR INSULAR AREA
}

\author{
ARKANSAS \\ Plants \\ ArborGen-Fred C Gragg SuperTree Nursery \\ Arkansas Forestry Commission-Baucum Nursery \\ Ozark Wildflower Company \\ Pine Ridge Gardens \\ Weyerhaeuser Company_Magnolia Nursery \\ Seeds \\ Holland Wildflower Farm \\ BRITISH COLUMBIA \\ Plants \\ A'Qam Native Plant Nursery \\ BC's Wild Heritage Plants \\ Birch Creek Nursery \\ BLUESTEM NURSERY \\ Dry Valley Nurseries \\ Fraser's Thimble Farms \\ Gabriola Growing Company \\ Green by Nature \\ K\&C Silviculture Farms Ltd \\ Keefer Ecological Services Ltd \\ Linnaea Nurseries Ltd \\ Mayo Creek Gardens \\ Mosterman Plant Propagators \\ Murray Nurseries Ltd \\ NATS NURSERY LTD \\ The Natural Gardener Garden Store \\ Natural Habitat Gardens \\ Natural Resource Native Plant Nursery \\ Pacific Rim Native Plants Ltd \\ PEEL'S NURSERIES LTD \\ Pelton Reforestation Ltd \\ Perennial Gardens \\ PRT Vernon Nursery \\ Shady Creek Native Plants and \\ Environmental Consulting \\ Streamside Native Plants
}

Tipi Mountain Native Plants

West Kootenay Plants

Seeds

Birch Creek Nursery

Linnaea Nurseries Ltd

The Natural Gardener Garden Store

Natural Legacy Seed

Natural Resource Native Plant Nursery

Nature's Garden Seed Company

Pacific Rim Native Plants Ltd

Quality Seed Collections

Quality Seeds West

SYMBIOS RESEARCH AND RESTORATION

Yellowpoint Propagation Ltd

\section{CALIFORNIA}

\section{Plants}

Antelope Valley Resource Conservation District Tree Nursery \& Arboretum

Bay Natives

Boething Treeland Farms Inc

Brokaw Nursery

Cal-Forest Nurseries

Calaveras Nursery

California Dept of Forestry and Fire Protection-

Magalia Reforestation Center

California Flora Nursery

Canfield Native Nursery

Capitol Wholesale Nursery Inc

Carmel Valley Seed Company

Catalina Island Conservancy-

James H Ackerman Native Plant Nursery

The Center for Social and Environmental

Stewardship (formerly Circuit Rider

Productions Inc)

Central Coast Wilds (see ad on page 161)

CORNFLOWER FARMS (see ad on page 165)

Creekside Gardens

\author{
Digging Dog Nursery \\ Eastern Sierra Native Plants \\ EL NATIVO GROWERS INC \\ Elkhorn Native Plant Nursery \\ Far West Bulb Farm \\ FRESHWATER FARMS \\ Gaspars Nursery \\ Georgia Pacific West Inc-Forest Tree Nursery \\ Glass Mountain Forest Tree Nursery \\ Go Native Nursery \\ Golden State Growers \\ Green Desert Wholesale Nursery \\ Green Oak Branch Nursery \\ Greenlee Nursery \\ GroWest Nurseries \\ GROWING SOLUTIONS RESTORATION \\ EDUCATION INSTITUTE \\ Habitat Restoration \\ HARTLAND NURSERY (see ad on page 177) \\ Hastings Smith River Nursery \\ High Ranch Nursery \\ Hines Hort Nursery \\ IFA Nurseries Inc- \\ Humboldt Nursery \\ Intermountain Nursery \\ Jeff Anhorn Nursery \\ JM Oak Tree Nursery \\ Las Pilitas Nursery-Escondido \\ Las Pilitas Nursery_-Santa Margarita \\ LE Cooke Company \\ Little River Nursery \\ Live Oak Nursery \\ Manzanita Native Plant Nursery \\ Matilija Nursery \\ Menzie's Native Nursery \\ Middletown Rancheria \\ Mockingbird Nurseries Inc \\ Monterey Bay Nursery
}


Moon Mountain Wildflowers

Moosa Creek Nursery

Mostly Natives

Napa Native Plant Nursery

Native Here Nursery

Native Revival Nursery

Native Sons Inc

Native Springs Nursery

Nature's Image Inc

NORTH COAST NATIVE NURSERY

Northwest Native Seed

O'Donnells Fairfax Nursery

Pechanga Band of Luiseno Indians

Performance Nursery

Phelan Garden I DBA Phelan Corporation

Plants for Dry Places

Quail Botanical Gardens Foundation Inc

RECON NATIVE PLANTS INC

Redwood Valley Rancheria

Regional Parks Botanic Garden

Restoration Resources

The Reveg Edge

Robinson Rancheria

San Marcos Growers

Santa Barbara Botanic Garden

Shasta Plantation

Shelterbelt Builders Inc

Sierra Valley Farms

Simpson Timber Company_Korbel Forest Nursery

Siskiyou Tree \& Mountain

Smith River Nursery-Hastings LLC

Star Nurseries

Suncrest Nurseries Inc

Sunset Coast Nursery

Tahoe Tree Company

Tarweed Native Plant Nursery \& Landscape

Tarweed Nursery and Landscape

Telos Rare Bulbs

Temecula Band of the Luiseno Indians

Theodore Payne Foundation Nursery

Tiedemann Nursery

TREE OF LIFE NURSERY (see ad on page 225)

Tree Foundation of Kern

Tsemeta Forest Nursery

Tuolumne Mi-Wuk Native Plants

USDA Forest Service-Genetic Resource and

Conservation Center

USDA Forest Service-Placerville Nursery

USDI National Park Service-Joshua Tree National

Monument Center for Arid Lands Restoration

USDI National Park Service-Sequoia \& Kings

Canyon National Parks Native Plant Nursery

Village Nurseries

The Villager Nursery

The Watershed Nursery

Wildwood Farm

Yerba Buena Nursery

Seeds

The Acorn Seed

Bundy CanyonTurf Supply

California Dept of Forestry and Fire ProtectionLA Moran

Carmel Valley Seed Company

Central Coast Wilds (see ad on page 161)

County of Los Angeles Fire Dept

Environmental Seed Producers

Floral Native Nursery Inc
Forest Seeds of California

FRESHWATER FARMS

Freshwater Farms I North Coast Native Seed Bank Georgia Pacific West Inc-Forest Tree Nursery

GROWING SOLUTIONS RESTORATION

EDUCATION INSTITUTE

IL Hudson, Seedsman

LA Moran Reforestation Center

Larner Seeds

Magalia Reforestation Nursery

Mistletoe Carter Wholesale Seed

Mourning Cloak Ranch \& Botanical Gardens

Northwest Native Seed

O'Donnells Fairfax Nursery

Pacific Coast Seed

RECON NATIVE PLANTS INC

Redwood City Seed Company

S\&S Seeds I Albright Seed Company

Seedhunt

Sierra Seed Supply

Stover Seed Company

Theodore Payne Foundation Nursery

TREE OF LIFE NURSERY (see ad on page 225)

USDI National Park Service-Sequoia \& Kings

Canyon National Parks Native Plant Nursery

The Wildflower Seed Company

\section{COLORADO}

Plants

Applewood Seeds

Aquatic and Wetland Company

Boxelder Creek Nursery

Chelsea Nursery

COLORADO STATE FOREST SERVICE

NURSERY

Dry West Nursery LLC

Evergreen Nursery

Fossil Creek Nursery

Native Nursery

Pagosa Nursery Company

Palisade Greenhouse

PLEASANT AVENUE NURSERY INC

Rocky Mountain Native Plants Company

Rocky Mountain Pines

Sunscapes

Tagawa Garden Center \& Florist

USDA Natural Resources Conservation Service-

Upper Colorado Environmental Plant Materials Center

USDI Bureau of Indain Affairs-Southern Ute Agency

USDI National Park Service-

Rocky Mountain National Park Native Plant

Nursery

Valdez Native Plants

Western Native Seed

Seeds

Applewood Seeds

Arkansas Valley Seed Solutions

AV Seeds

Beauty Beyond Belief

Colorado Seed Solutions

Pawnee Buttes Seed Inc

Rocky Mountain Rare Plants

Southwest Seed Inc
USDA Natural Resources Conservation ServiceUpper Colorado Environmental Plant Materials Center

Western Native Seed

CONNECTICUT

Plants

Broken Arrow Nursery

Cricket Hill Garden

Earth Tones Native Plant Nursery

Millane Nurseries

New England Environmental Services

PAN'S ACRES NURSERY

Prides Corner Farms

Silk Tree Gardens

Summer Hill Nursery

Sunny Border Nurseries Inc

White Flower Farms

Seeds

Chas C Hart Seed Company

\section{DELAWARE}

Plants

Delaware Native Plant Society

Phillips Nurseries Inc

Shelterwood Farm

\section{FLORIDA}

Plants

3E TREE FARMS AND WETLAND NURSERY INC A Quality Plant

ACTION THEORY LANDSCAPE AND NURSERY

Alexander Landscaping \& Plant Farms

All Native Garden Center \& Plant Nursery

All Natives

American Native Products

Andrews Nursery Florida Division of Forestry

Apalachee Native Nursery

AQUATIC PLANTS OF FLORIDA INC

(see ad on page 151)

Aquatic Systems \& Resources

ArborGen-SuperTree Orchards

Arvida Nurseries

Bartow Ornamental Nursery

Bate's Plants

Beeman's Nursery Inc

Biosphere Consulting Inc

Black Creek Nursery

Bloomers Flowering Plant Nursery

BOTANICS WHOLESALE INC

Boynton Botanicals

Breezy Oaks Nursery

Buckeye Nursery Inc

Carencia Native Nursery

Central Florida Lands and Timber

CHIAPPINI FARM NATIVE NURSERY

CNPS Inc

Concepts in Greenery Inc

Deluxe Trees and Shrubs

DR Bates

Dwight Stansel Farm \& Nursery

EarthBalance

The Echo Center

Echo Nursery

Environmental Equities Inc

Finders Keepers Plants

Florida Aquatic Nurseries Inc 
Florida Division of Forestry_Andrews Nursery

Florida Keys Native Nursery

Florida Native Flora Inc

Florida Native Plants Inc

Foreverflora Palm Nursery

The Garden Gate

Garden of Delights

GONE NATIVE NURSERY

Grandiflora I San Felasco Nurseries Inc

Green Images Native Landscape Plants

Green Isle Gardens

Green Seasons Nursery

Halfmoon Growers Inc

Hard Scrabble Farms Inc

Hickory Hill Native Nursery Inc

Horticultural Systems Inc

Indian Trails Native Nursery

Liner Farm Inc

Mail-Order Natives

Maple Street Natives

Marshall Tree Farms

Matlack Tree Farm

McKeithen Growers Inc

Meadow Beauty Nursery

Mesozoic Landscapes Inc

Native and Uncommon Plants

Native Nurseries of Tallahassee Inc

Native Plant Growers

Native Technologies Inc

Native Tree Nursery Inc

THE NATIVES INC

Nick's Nursery Inc

Norman's Native Plants Plus

Oasis Tree Farms

Ornamental Plants and Trees Inc

Pat Ford's Nursery Inc

Pelton's Nursery Inc

PERKINS NURSERY INC

Pine Breeze Nursery

Plant Creations Inc

Rancho La Orquidea Inc

Reflections of Nature

Rigsby Nursery Inc

RSS Field Services Inc

Runway Growers Inc

San Felasco Nurseries Inc

Sebastian River Ranch LLC

SeedClean

Shadowlawn Nursery

Simonton Farms Nursery

Soaring Eagle Nursery

Southeast Trees I Bartow Ornamental Nursery

Southern Horticulture

Southern Native Nursery Inc

Southern Native Plants LLC

Southern Pride Tree Farm Inc

Sturon Inc

SUNCO

Sundance Ornamentals

Superior Trees

Trail Ridge Nursery

Trans Gro Superior Plant Company Division

TreeMart

Tropic Traditions Nurseries

Tropical Plant and Seed Nursery

University of Florida-Center for Aquatic and Invasive Plants
Urban Forestry Services

Vila \& Son Nursery Corporation

Whisper Palms of Terra Ceia LLC

Wilcox Nursery

Seeds

Biosphere Consulting Inc

Dwight Stansel Farm \& Nursery

Hard Scrabble Farms Inc

Native Nurseries of Tallahassee Inc

THE NATIVES INC

Pat Ford's Nursery Inc

PERKINS NURSERY INC

SeedClean

Tropical Plant and Seed Nursery

Urban Forestry Services

\section{GEORGIA}

\section{Plants}

ArborGen-Bellville SuperTree Nursery

ArborGen-Georgia SuperTree Nursery

ATS PARTNERS LLC (see ad on page 153)

Bell Brothers Inc

Carters Nursery-Bowater Forest Products Division

Eco Gardens

Garden Delights LLC I Lazy K Nursery Inc

Goodness Grows Inc

INTERNATIONAL FOREST COMPANY

John Deere Landscapes

Meeks Farms

Mountain Valley Farms

Native Forest Nursery

Plum Creek Timber Company-Jesup Nursery

Randy's Perennials \& Water Gardens

Rayonier Inc

Rock Spring Farm

Rutland Forest Nursery

Southern Roots Tree Nursery

Transplant Nursery

Walker Nursery Farms

Seeds

ATS PARTNERS LLC (see ad on page 153)

Flint River Nursery

INTERNATIONAL FOREST COMPANY

Mobley Greenhouse Inc

Plum Creek Timber Company-Jesup Nursery

Rutland Forest Nursery

GUAM

Plants

Guam Dept of Agriculture-Forestry Division

\section{HAWAI'}

\section{Plants}

Aikane Nursery

Aileen's Nursery-Po'o Wai U

Amy Greenwell Ethnobotanical Gardens Nursery

Charles Nii Nursery

E Nakashima Greenhouses

Future Forests Nursery

Hawai'i Division of Forestry \& WildlifeHilo Tree Nursery

Hawai'i Division of Forestry \& WildlifeKamuela State Tree Nursery

Hawai'i Division of Forestry \& WildlifeKaua'i District Nursery

Hawai'i Division of Forestry \& WildlifeKokee State Park Native Plant Nursery
Hawai'i Division of Forestry \& Wildlife-

Maui District Nursery

Hawai'i Division of Forestry \& Wildlife-

Rare Plant Facility

Hawaii Palm Company

Hawaiian Gardens

Ho'olawa Farms

lo Makuahine

Kapoho Kai Nursery

Kauai Nursery and Landscaping Inc

Makani Gardens

Maui Native Nursery

National Tropical Botanical Garden

Native Nursery LLC

Sunrise Nursery

University of Hawai'i at Manoa-Harold L Lyon Arboretum

US Fish \& Wildlife Service-Hakalau Forest Nationa Wildlife Refuge Native Plant Nursery

US Fish \& Wildlife Service-Kilauea Lighthouse National Wildlife Refuge Native Plant Nursery USDI National Park Service-Haleakula National

Park Native Plant Nurseries

USDI National Park Service-Hawai'i Volcanoes

National Park Native Plant Nursery

USDI National Park Service-Kalaupapa National Historic Park Native Plant Nursery

Waimea Valley Arboretum and Botanical Garden

Seeds

Hawai'i Division of Forestry \& Wildlife-Kamuela State Tree Nursery

Hawaii Reforestation Company

Kauai Nursery and Landscaping Inc

Waimea Valley Arboretum and Botanical Garden

\section{IDAHO}

Plants

BUFFALO-BERRY FARM

Conservation Seeding \& Restoration Inc

David R Mossman Ranch Inc

Draggin' Wing Farm, Water-Thrifty Plants for Idaho

Edwards Greenhouse

The Green Ranch

Intermountain Aquatics

JAYKER WHOLESALE NURSERY INC

Jenkins \& Son Tree Farm

Moss Greenhouses

Native and Xeric Plants Inc

Native Landscapes Inc

Native Sod Solutions

NATIVESWEST NURSERY \& ECO-SERVICES LLC

(see ad on page 197)

North Woods Nursery Inc

Palouse-Clearwater Environmental Institute (PCEI)

Learning Nursery

Plantasia Cactus Gardens

Trail Creek Nursery Inc

UNIVERSITY OF IDAHO_FRANKLIN H PITKIN FOREST NURSERY

USDA Forest Service-Coeur d'Alene Nursery

USDA Forest Service-Lucky Peak Nursery

Webb Landscape Inc

Western Forest Systems Inc

WILDLIFE HABITAT NURSERY 


\section{Seeds}

BUFFALO-BERRY FARM

Cedera Seed Inc

Clifty View Nursery

Conservation Seeding \& Restoration Inc

Edwards Greenhouse

Ford Seed Company Inc

Idaho Grimm Growers

NATIVE SEED FOUNDATION

Oregon-Idaho Native Plant Seed Growers

Association

PINEVIEW HORTICULTURAL SERVICES

Plantasia Cactus Gardens

Seed Specialists Inc

Sun Mountain Natives

THORN CREEK NATIVE SEED FARM

USDA Forest Service-Lucky Peak Nursery

\section{ILLINOIS \\ Plants}

Anna Nursery

Aquatic Nursery

Blazing Star Associates

Bluestem Prairie Nursery

Bohm's Garden Center

COUNTRY ROAD GREENHOUSES INC

Elmer Bailey Nursery

Enders Greenhouse

GENESIS NURSERY INC (see ad on page 173)

Gerard \& Greene

Glacier Oaks Nursery

HE Nursery

Illinois Division of Forest Resources-

Mason State Tree Nursery

Illinois Division of Forest Resources-

Union State Tree Nursery

Illinois Forest Products

King Nursery

McHenry County Nursery Inc I

Glacier Oaks Native Nursery

THE NATURAL GARDEN INC

Naturescapes Nursery

Possibility Place Nursery

PRAIRIE EARTH NURSERY

Purple Prairie Farm

Simply Native Nursery

Vienna Nursery

Seeds

Blazing Star Associates

Bluestem Prairie Nursery

Bluff Creek Farm

Earthskin Nursery

GENESIS NURSERY INC (see ad on page 173)

Illinois Division of Forest Resources-

Mason State Tree Nursery

Lafayette Home Nursery Inc

THE NATURAL GARDEN INC

Peter Schramm—Prairie Restorations

PRAIRIE EARTH NURSERY

Purple Prairie Farm

Sand Prairie Farms Inc

Wilson Seed Farms

\section{INDIANA}

Plants

Beineke's Nursery

Blank's Nursery, Garden \& Pet Center
Edge of the Prairie Wildflowers

Heartland Restoration Services I Earth Source Inc

Henseler Nursery Inc

Indiana Division of Forestry-Jasper-Pulaski State

Nursery

Indiana Division of Forestry—Vallonia State Nursery

IF NEW NATIVE PLANT NURSERY

(see ad on page 183)

THE LAND NURSERY

Munchkin Nursery and Gardens LLC

SPENCE RESTORATION NURSERY

Water Resources Design Inc

WINTERHAVEN WILDFLOWERS \& NATIVE

PLANT PRESERVE (see ad on page 235)

Woody Warehouse Nursery

Seeds

Earthly Goods Ltd

Heartland Restoration Services I Earth Source Inc

JF NEW NATIVE PLANT NURSERY

(see ad on page 183)

SPENCE RESTORATION NURSERY

Thompson \& Morgan Seedsmen Inc

IOWA

Plants

CASCADE FORESTRY NURSERY

Country Landscapes Inc

Diversity Farms

Greenworld Inc

IAWISIL Forest Nursery

lon Exchange Inc

lowa Dept of Natural Resources-

State Forest Nursery

Kingfisher Farm

Mount Arbor Nursery

Murphy's Walnut Hill Nursery Inc

Reeves Wildflower Nursery

Renee's Garden

Rose Hill Nursery

UNIVERSITY OF NORTHERN IOWA-

TALLGRASS PRAIRIE CENTER

(see ad on page 227)

Seeds

Allendan Seed (see ad on page 149)

CASCADE FORESTRY NURSERY

Heyne Custom Seed Services

Ion Exchange Inc

Kingfisher Farm

Osenbach Grass Seed

Reeves Wildflower Nursery

Renee's Garden

Swanson Farms

UNIVERSITY OF NORTHERN IOWA-

TALLGRASS PRAIRIE CENTER

(see ad on page 227)

\section{KANSAS}

Plants

Kansas Forest Service

Kaw River Restoration Nurseries

NPK Nursery

Sunflower Farms

Vinland Valley Nursery

Seeds

DeLange Seed Inc

Kaw River Restoration Nurseries

Sharp Bros Seed Company

\section{KENTUCKY}

Plants

Dropseed Native Nursery

HABITATS NATIVE PLANT NURSERY

Kentucky Division of Forestry-

John P Rhody Nursery

Kentucky Division of Forestry-

Morgan County Nursery

Nolin River Nut Tree Nursery

Shooting Star Nursery

Wearren and Sons Nurseries Inc

Seeds

Dropseed Native Nursery

HABITATS NATIVE PLANT NURSERY

Jane's Native Seeds

M\&M Native Grass Seed Company

LOUISIANA

Plants

The Bosch Nursery Inc

Clifton-Choctaw Nursery

Coyote Creek Inc

Durio Nursery

ECOLAGE Purveyors of Wonder

Louisiana Growers

Northeast Delta RC\&D Hardwood Seedling

Nursery

Pushpetappa Gardens

Seeds

Louisiana Dept of Agriculture \& Forestry

Louisiana Dept of Agriculture \& Forestry-

Columbia Nursery

Louisiana Forest Seed Company

(see ad on page 189)

MAINE

Plants

ArborGen-Forest Resources Hermon Nursery

Aroostook Band of Mic Macs

Fieldstone Gardens Inc

Johnny's Selected Seeds

Lawrence Mountain Nurseries

Native Haunts

Old Ridge Nursery

PIERSON NURSERIES INC

Western Maine Nurseries

Seeds

Johnny's Selected Seeds

Native Haunts

PIERSON NURSERIES INC

MANITOBA

Seeds

Blight Native Seeds Ltd

INTERLAKE FORAGE SEEDS LTD

Prairie Habitats Inc

Tamarack Farms

MARYLAND

Plants

ADKINS ARBORETUM

AMERICAN NATIVE PLANTS

American Native Plants

Angelica Nurseries Inc

The Behnke Nurseries Company

Chesapeake Native Nursery

Clear Ridge Nursery Inc 
Elk Ridge NatureWorks LLC

ENVIRONMENTAL CONCERN INC

(see ad on page 169)

Homestead Gardens

KOLLAR NURSERY

(see ad on page 187)

Lilypons Water Gardens

Lower Marlboro Nursery

Maryland Aquatic Nurseries

Maryland Department of Natural ResourcesJohn S Ayton State Tree Nursery

Maryland Natives Nursery

Raemelton Farm

Wicklein's Water Gardens

Seeds

Chesapeake Native Nursery

Maryland Department of Natural ResourcesJohn S Ayton State Tree Nursery

Native Seeds Inc

\section{MASSACHUSETTS \\ Plants \\ Blanchette Gardens \\ USDA Forest Service-JW Toumey Nursery \\ Wildtype Design Native Plants \& Seed Ltd \\ Windy Hills Farm}

Cavicchio Nurseries

Donaroma's Nursery

Hillside Nursery

NEW ENGLAND WETLAND PLANTS

NEW ENGLAND WILD FLOWER SOCIETY

(see ad on page 199)

Project Native

Tripple Brook Farm

Underwood Shade Nursery

Seeds

FW SCHUMACHER COMPANY INC

GARDENMAKERS

NEW ENGLAND WETLAND PLANTS

NEW ENGLAND WILD FLOWER SOCIETY

(see ad on page 199)

Project Native

MICHIGAN

Plants

Abbott's Nursery

Alpha Nurseries

Arrowhead Alpines

Bosch's Countryview Nursery

Cold Stream Farm

Engel's Nursery Inc

Great Lakes Nursery Company

Hartmann's Plant Company

Hramor Nursery

John Arnoldink Nursery

Lake Superior Tree Farms

Lodi Farms Nursery

Mathisen Tree Farms

Michigan Dept of Natural Resources-

Wyman State Forest Nursery

Michigan Wildflower Farm

The Native Plant Nursery

Needlefast Evergreens Inc

Newpage Corporation

Oikos Tree Crops

Ole Henry Gardens

Ponderosa Nursery

Sandhill Farm

Stempky Nursery

USDA Forest Service—JW Toumey Nursery
USDA Forest Service-Ottawa National Forest

USDA Natural Resources Conservation Service-

Rose Lake Plant Materials Center

Vans Pines Nursery Inc

Wetlands Nursery

Wildtype Design Native Plants \& Seed Ltd

Zelenka Evergreen Nursery

\section{Seeds}

Bosch's Countryview Nursery

Engel's Nursery Inc

JW Toumey Nursery

Kobe Nurseries

Michigan Wildflower Farm

Native Connections

The Native Plant Nursery

Needlefast Evergreens Inc

New Life Nursery Inc

Newaygo Conservation District Nursery

Northern Pines Nursery

Peterson's Riverview Nursery LLC

\section{MINNESOTA}

Plants

Busse Gardens

ITASCA LADYSLIPPER FARM I

SPANGLE CREEK LABS

KASTE SEED INC (see ad on page 187)

Kneght's Nurseries

Landscape Alternatives

Mineland Reclamation Division

Minnesota Native Landscapes

Minnesota State Forest Nurseries-

Minnesota Dept of Natural Resources

Minnesota Valley Garden Center

MORNING SKY GREENERY

Natural Shore Technologies Inc

Nature's Acres I Minnesota Native Landscapes

North American Prairies Company

North Central Reforestation Inc

Northern Lights Silviculture

Outback Nursery Inc

Plantra Inc

Prairie Hill Wildflowers

PRAIRIE MOON NURSERY

Prairie Restorations Inc (see ad on page 211)

SCHUMACHERS NURSERY \& BERRY FARM INC

Spring Lake Restoration Nurseries

Sunshine Gardens Nursery and Landscaping Inc

USDI Bureau of Indian Affairs-

Red Lake Forestry Greenhouse

Seeds

Carlson Prairie Seed Farm Inc

(see ad on page 159)

FEDER PRAIRIE SEED COMPANY

Itasca Greenhouse Inc

ITASCA LADYSLIPPER FARM I

SPANGLE CREEK LABS

KASTE SEED INC (see ad on page 187)

Mark E Gullickson

Mohn Seed Company

Nature's Acres I Minnesota Native Landscapes

Norfarm Seeds Inc

North American Prairies Company

Prairie Hill Wildflowers
PRAIRIE MOON NURSERY

Prairie Restorations Inc (see ad on page 211)

SHOOTING STAR NATIVE SEEDS

(see ad on page 217)

Spring Lake Restoration Nurseries

\section{MISSISSIPPI}

Plants

Delta-View Nursery

Mississippi State University-Coastal Research and

Extension Center

Mississippi State University-The Crosby

Arboretum

Natchez Trace Gardens

Plum Creek Timber Company-Pearl River Nursery

Plum Creek Timber Company-Shubuta Nursery

Strawberry Plains Audubon Center

Seeds

Plum Creek Timber Company_Pearl River Nursery

\section{MISSOURI}

Plants

A Waldbart \& Sons Nursery

Bowood Farms Inc

FORREST KEELING NURSERY INC

(see ad on page 171)

Hamilton Native Outpost

Hartke Nursery

Heartland Nursery Company

Jim's Garden Center

Longfellow's Garden Center

Midwest Cactus

MISSOURI DEPT OF CONSERVATION-

GEORGE O WHITE STATE FOREST NURSERY

MISSOURI WILDFLOWERS NURSERY LLC

One Garden I Elixir Farm Botanical

Osage Prairie Mercantile

Prairie Hill Farm

Ripley County Farms

Total Resource Management

Seeds

Hamilton Native Outpost

Lovelace Seeds Inc

MISSOURI WILDFLOWERS NURSERY LLC

One Garden I Elixir Farm Botanical

Reed's Seeds Inc

Sharp Bros Seed Company of Missouri

Total Resource Management

\section{MONTANA}

Plants

Billings Nursery \& Landscaping

Bitterroot Restoration Inc

Blackfeet Community College Greenhouse

BLACKFOOT NATIVE PLANTS NURSERY

Caras Nursery \& Landscape

CS\&KT Forestry Tribal Nursery

Glacier Nursery Inc

GREAT BEAR RESTORATION

Grouse Springs Nursery

LAWYER NURSERY INC MT

MONTANA DEPT OF NATURAL RESOURCES \&

CONSERVATION-MONTANA

CONSERVATION SEEDLING NURSERY

Native Ground

NATURE'S ENHANCEMENT INC 
OASIS Environmental Native Nursery

Plum Creek Timber Company-

Pablo Montana Forest Nursery

Salish \& Kootenai Tribal College Native

Plant Nursery

USDI National Park Service-

Glacier National Park Native Plant Nursery

Valley Nursery

Westscape Wholesale Nursery

Seeds

Circle S Seeds of MT Inc

GREAT BEAR RESTORATION

LAWYER NURSERY INC MT

Treasure State Seed Inc

USDI National Park Service-Glacier National Park

Native Plant Nursery

Westland Seed Inc

\section{NEBRASKA}

Plants

Bluebird Nursery

Garden Perennials

Nature Hills Nursery Inc

TODD VALLEY FARMS

USDA Forest Service-

Nebraska National Forest \& Grasslands

Seeds

Arrow Seed Company Inc

PE Allen Ranch Supply

Stock Seed Farms

USDA Forest Service-Bessey Nursery

\section{NEVADA}

Plants

Cactus Joe's Blue Diamond Cactus Nursery

Duckwater-Shoshone Nursery

Found Well Farm

Native Oak Nursery Supply

Native Plant Farm \& Tree Movers

Nevada Division of Forestry-Las Vegas Nursery

Nevada Division of Forestry-Washoe Nursery

Seeds

Comstock Seed

Native Plant Farm \& Tree Movers

Nevada Division of Forestry-Washoe Nursery

\section{NEW BRUNSWICK}

Plants

JD Irving Juniper Nursery

NEW HAMPSHIRE

Plants

ELFS Landscaping Inc

ROLLING GREEN NURSERY

Uncle Tim's Farm \& Garden Center

Van Berkum Nursery

Yankee Gardener Greenhouses and Nursery

Seeds

New Hampshire State Forest Nursery

\section{NEW JERSEY}

Plants

Arrowwood Native Plant Nursery LLC

Cicconi Farms Inc I The Perennial Specialists

Clemenson Farms Native Nursery

Coastal Native Plants Nursery LLC

Fairweather Gardens
Mapleton Nurseries

New Jersey Division of Parks and Forestry-

Forest Tree Nursery

New Moon Nursery LLC (see ad on page 199)

Pinelands Nursery Inc

Springbrook Farms

Toadshade Wildflower Farm

Waterford Gardens

Seeds

Arrowwood Native Plant Nursery LLC

Croshaw Nursery

NEW MEXICO

Plants

Agua Fria Nursery

Bernado Beach Native Plant Farm

Cassidy Farm Nursery

Helen's Native Plants \& Rustic

HIGH COUNTRY GARDENS

Hydra Aquatic

New Mexico Forestry Division

New Mexico State University-

Mora Research Center

Pajarito Greenhouse

Plant Propagation Technologies Inc

Plants of the Southwest

Ramah Navajo Community Enterprise Inc-

Native Plants Nursery

Santa Ana Garden Center

Santa Fe Greenhouse Inc

Sierra Vista Growers

Trees of Corrales

Trees That Please

USDI Bureau of Indian Affairs-Forest Development

USDI Bureau of Indian Affairs-Jicarilla Agency

USDI Bureau of Indian Affairs-

Southwestern Indian Polytechnic Institute

Seeds

Forest Development Bureau of Indian Affairs

Mesa Garden

Plants of the Southwest

Santa Fe Greenhouse Inc

Seeds West Garden Seeds

\section{NEW YORK}

Plants

Amanda's Garden

Catskill Native Nursery

City of New York Parks and Recreation-

Greenbelt Native Plant Center

Fiddlehead Creek

Fort Pond Native Plants

Native Landscapes Garden Center

New York State Dept of Environmental

Conservation-Saratoga Tree Nursery

Pinelands Nursery Inc

Seneca Hill Nursery

Southern Tier Consulting and Nursery Inc

St Lawrence Nurseries

TREEHAVEN EVERGREEN NURSERY ARBORETUM ON BLUE HILL

Seeds

Fiddlehead Creek

IIM HARRINGTON SEEDS

New York State Dept of Environmental

Conservation-Saratoga Tree Nursery

Pinelands Nursery Inc
NORTH CAROLINA

Plants

Burgaw Creek Nursery \& Turf Farm LLC

Carolina Greenery

Carolina Native Nursery

Coastal Plain Conservation Nursery

COASTAL PLAIN CONSERVATION NURSERY INC

Cure Nursery

Darwin's Backyard Nursery

Elk Mountain Nursery

Gardens of the Blue Ridge

GHW Weyerhaeuser Nursery

Hanging Dog Valley Nursery

MEADOWBROOK NURSERY I WE-DU NATIVES

Mellow Marsh Farm

Niche Gardens

North Carolina Division of Forest Resources-

Claridge Nursery

North Carolina Division of Forest ResourcesLinville River Nursery

Plant Delights Nursery

Wildflower Nursery

Wilson Greenery

Yellow Mountain Farms

\section{NORTH DAKOTA}

Lincoln-Oakes Nurseries

North Dakota State Forest ServiceTowner State Nursery

\section{NOVA SCOTIA}

Seeds

Nova Scotia Dept of Natural ResourcesStrathlone Forest Nursery

$\mathrm{OHIO}$

Plants

BC Nursery

Bluestone Perennials Inc COMPANION PLANTS INC

Envirotech Nursery

Mary's Plant Farm \& Landscaping

Naturally Native Nursery

OHIO DIVISION OF FORESTRY MARIETTA STATE NURSERY

Riverside Native Trees

Scioto Gardens

Smith Evergreen Nursery Inc

Seeds

COMPANION PLANTS INC

Mary's Plant Farm \& Landscaping

OHIO DIVISION OF FORESTRY -

MARIETTA STATE NURSERY

Ohio Prairie Nursery

\section{OKLAHOMA}

Plants

Greenleaf Nursery-Oklahoma

Martin Perennial Farms Inc

Sunshine Nursery

Seeds

Grasslander

Johnston Seed Company

Oklahoma Dept of Agriculture-

Albert Engstrom Forest Regeneration Center 


\section{ONTARIO}

Plants

ACORUS RESTORATION

Native Plants in Claremont

North Sun Nurseries Inc

Ontario Native Land

Van Dan Nest Nursery

WILD Canada

Wild Ginger Native Plant Nursery

Wildflower Farm Inc

\section{Seeds}

ACORUS RESTORATION

Native Plant Source

Wheatley Woods Native Plant Nursery and Garden Centre

Wildflower Farm Inc

\section{OREGON}

\section{Plants}

Adams-Franklin Tree Seedlings Nursery

Alpha Nursery Inc

Althouse Nursery

Aurora Nursery Inc

BALANCE RESTORATION NURSERY LLC

(see ad on page 153)

Barth Nursery

BeaverLake Nursery

Big Trees Today

Bloom River Gardens

Bosky Dell Natives

Brooks Tree Farm

Caprice Farm Nursery

Carter's Greenhouse \& Nursery Inc

CHAMPOEG NURSERY INC

Clearwater Native Nursery

Confederated Tribes of the Umatilla Indian Reservation

Cousin's Nursery

Curry Native Plants

D Wells Farms

Doak Creek Native Plant Nursery

Drakes Crossing Nursery

Eby Nursery Inc

ECEC Native Plant Nursery

Echo Valley Natives

Fernwood Nursery

Ferris Nursery

Flora Lan Nursery

Forestfarm

Goodwin Creek Gardens

Green Hills Nursery

Greer Gardens

Harold M Miller Landscape Nursery

HERITAGE SEEDLINGS INC (see ad on page 179)

Holden Wholesale Growers

HORIZON HERBS LLC

Hoyt Arboretum

Huckleberry Lane Nursery

Hughes Water Gardens

Humble Roots Farm and Nursery LLC

IFA Nurseries Inc-Canby Forest Nursery

IFA Nurseries Inc-Corporate Office

IFA Nurseries Inc-Klamath Falls Forest Nursery

J Frank Schmidt \& Son Company

Jansen's Specialty Nursery

Joy Creek Nursery
Karma's Forest Wholesale Nursery and

Organic Gardens

Kendrick Forest Farm

King Estate I DBA Lorane Grape Vines

Kintigh's Mountain Home Ranch

Klamath Forest Nursery

Krueger's Tree Farms

Lava Nursery Inc

Legacy Land Conservancy

Lone Elder Nursery

Mahonia Vineyards \& Nursery Inc

Minto Island Growers

Native Grounds Nursery

Native Habitat Nursery

Northwest Native Plants Inc

Oak Point Nursery

Oakhill Farms Native Plant

Oregon Native Plant Nursery

Pacific Northwest Natives (see ad on page 205)

Plant Oregon (see ad on page 207)

The Plantworks LLC

Plum Creek Timber Company-

Cottage Grove Nursery

Portland Nursery

Postlewait Nursery

Prindel Creek Farm Inc

PRT Oregon Nursery

Red's Rhodies

Rock Bottom Ranch Koi and Nursery

Roseburg Forest Products-Lebanon Forest

Regeneration Center

Rugged Country Plants

Sauvie Island Ornamentals LLC

Scholls Valley Native Nursery LLC

SECOND GROWTH INC

SEVENOAKS NATIVE NURSERY LLC

Silver Mountain Nursery

Silver Springs Nursery Inc

Siskiyou Rare Plant Nursery

Sylvan Options

TRILLIUM GARDENS (see ad on page 227)

USDA Forest Service-J Herbert Stone Nursery

USDI Bureau of Land Management-

Charles A Sprague Tree Seed Orchard

USDI Bureau of Land Management-

Walter Horning Seed Orchard

Valley Growers

WALLACE HANSEN NATIVE PLANTS

Water Wheel Farms

Westlake Nursery at Farout Farm

Weyerhaeuser Company-Aurora Forest Nursery

Weyerhaeuser Company-Turner Nursery

Whitman Farms

Wichita Nursery Inc

Wild Rose Ranch

Willamette Gardens

WinterCreek Restoration

Woodsman's Native Plants

Seeds

Abundant Life Seed

Adams-Franklin Tree Seedlings Nursery

American Grass Seed Producers

Bailey Seed Company Inc

Callahan Seed

CHAMPOEG NURSERY INC

ECEC Native Plant Nursery

Emerald Seed and Supply
Ferris Nursery

HERITAGE SEEDLINGS INC

(see ad on page 179)

Hobbs and Hopkins

HORIZON HERBS LLC

J Frank Schmidt \& Son Company

Minto Island Growers

OBSIDIAN SEED COMPANY

(see ad on page 201)

OREGON WHOLESALE SEED

(see ad on page 203)

The Plantworks LLC

Plum Creek Timber Company-

Cottage Grove Nursery

Round Butte Seed Growers Inc

S\&S SEEDS I ENVIRONMENTAL SEED

PRODUCERS

SEVENOAKS NATIVE NURSERY LLC

Sunmark Seeds International Inc

Triangle Farms

USDA Forest Service-J Herbert Stone Nursery

USDI Bureau of Land Management-

Charles A Sprague Tree Seed Orchard

USDI Bureau of Land Management-

Walter Horning Seed Orchard

\section{PENNSYLVANIA}

\section{Plants}

Abraczinskas Nurseries Inc

Appalachian Nurseries Inc

AQUASCAPES UNLIMITED INC

Behmerwald Nursery

BOWMAN WILDFLOWER PRESERVE

Brandywine Conservancy

Carino Nurseries

Doyle Farm Nursery

Edge of the Woods Native Plant Nursery LLC

Fairview Evergreen Nurseries Inc

Flickingers Nursery

Gary's Perennials LLC

Habitat Solutions Inc

Hanchars Superior Trees

Heronswood Nursery

Johnston Nurseries

Mahanoy Valley Nurseries

Maple Hill Farms

Meadowood Nursery

Mid Atlantic Natives

Miles W Fry \& Son

Natural Landscapes Nursery

North Creek Nurseries Inc

Northeast Natives \& Perennials

OCTORARO NATIVE PLANT NURSERY

(see ad on page 203)

Pennsylvania Bureau of Forestry-Penn Nursery

Pennsylvania State Game CommissionHoward Nursery

Pikes Peak Nurseries

Plumline Nursery

The Primrose Path

Redbud Native Plant Nursery LLC

SANDY WILSON NATIVE PLANT \& AQUATIC

SW HORTICULTURAL SERVICES

Strathmeyer Forests Inc

Sugarbush Nursery

SYLVA NATIVE NURSERY \& SEED COMPANY 
Sylvania Native

Wetland Supply Company I Native Plant Nursery

YELLOW SPRINGS FARM

Seeds

Better Forest Tree Seeds

BOWMAN WILDFLOWER PRESERVE

Brandywine Conservancy

Doyle Farm Nursery

ERNST CONSERVATION SEEDS

(see ad on page 169)

Musser Forests Inc

Pine Grove Nursery Inc

SANDY WILSON NATIVE PLANT \& AQUATIC I

SW HORTICULTURAL SERVICES

SYLVA NATIVE NURSERY \& SEED COMPANY

Wetland Supply Company I Native Plant Nursery

\section{PRINCE EDWARD ISLAND}

Plants

Prince Edward Island Ministry of Forestry-

J Frank Gaudet Nursery

\section{QUEBEC}

\section{Plants}

Bechedor Inc

\section{RHODE ISLAND}

Plants

Confreda Greenhouses \& Farms

\section{SASKATCHEWAN}

Plants

Clearwater Greenhouses

Dieter Martin Greenhouse Ltd

Lakeshore Garden Centre

LAKESHORE TREE FARMS LTD

Lonewolf Native Plants and Herb Farm

Seeds

Wolfater Native Seeds

\section{SOUTH CAROLINA}

Plants

ArborGen-South Carolina SuperTree Nursery

Carolina Wild

Coastal Gardens \& Nursery

Faucette Tree Farm Inc

Shady Grove Plantation Nursery

South Carolina Forestry Commission-

Taylor Nursery

Weyerhaeuser Company—Quail Ridge Nursery

WOODLANDERS INC

\section{SOUTH DAKOTA}

Plants

Big Sioux Nursery Inc

\section{TENNESSEE}

Plants

Appalachian Native Plants Inc

Bert Driver Nursery

Boyd \& Boyd Nursery

GROWILD INC

High Country Nursery

Hillis Nursery Company Inc

Hills Creek Nursery (see ad on page 179)

Myers Cove Nursery Inc

NASHVILLE NATIVES LLC

Native Gardens
O'Neal Nursery

Our Bamboo Nursery

Sleepy Hollow Nursery

Sunlight Gardens Inc

Tennessee Dept of Agriculture-Delano Nursery

TN Nursery Company

Trees By Touliatos

Triangle Nursery Inc

WARREN COUNTY NURSERY INC

(see ad on page 233)

Seeds

NASHVILLE NATIVES LLC

WARREN COUNTY NURSERY INC

(see ad on page 233)

\section{TEXAS}

Plants

A Touch O' Green Landscape \& Garden LLC

Aldridge Nursery Inc

ArborGen-Livingston SuperTree Nursery

ArborGen-Richard O Barham SuperTree Nursery

Barton Springs Nursery

Bella Flora

Bluestem Nursery

Buchanan's Native Plants

Cambell Timberland Management

Casa Flora Inc

Countryside Trees

Desert Land Nursery

Dodds Family Tree Nursery

Doremus Wholesale Nursery

Enchanted Gardens

Gottlieb Gardens

Greenleaf Nursery-Texas

Hall Tree Services

Heep's Native Plant Nursery

Hill Country Natives

Husband Family Nursery

It's About Thyme Garden Center

Joshua's Native Plants \& Garden Antiques

Living Desert

Madrone Nursery

McNeal Growers

Miller Nursery \& Tree Company

Native Ornamentals

Native Tree Farm

NATIVES OF TEXAS

New World Botanical

Oasis Tree Farms

Oma's Garten Pflanzen

Peach Springs Nursery

Pearson's Tree Place

Plantation Discount Nursery

Plum Creek Timber Company_Vivian Nursery

Pots \& Plants Garden Center

Rancho Lomitas Native Plant Nursery

ROHDES NURSERY

Snider Nursery

Sunbelt Trees Inc

Texas Forest Service-West Texas Nursery

Texas Grown Plants

Texas Native Trees

Tropical Star Enterprises Inc

Weston Gardens in Bloom Inc

Wichita Valley Nursery

Winter Garden Nursery

Womack Nursery Company
Wrights Nursery

YUCCA DO NURSERY

Seeds

Bamert Seed Company

Bella Flora

BROWNING SEED INC (see ad on page 159)

Desert Land Nursery

Douglass King Company Inc

East Texas Seed Company

Foster Rambie Grass Seed

James Reneau Seed Company

McCormick Seeds

Native American Seed

Native Texas Nursery

NATIVES OF TEXAS

Plum Creek Timber Company-Vivian Nursery

Pots \& Plants Garden Center

Richardson Agco

Texas Forest Service-Indian Mound Nursery

Turner Seed Company Inc

Weston Gardens in Bloom Inc

Wildseed Farms

UTAH

Plants

The Cotton Mill I Star Nursery

Great Basin Natives

GREAT BASIN SEED

High Mountain Nursery

Intermountain Cactus

Intermountain Seed Company

Progressive Plants

Star Nursery

Sun Mountain Growers

USDI National Park Service-

Zion National Park Native Plant Nursery

Wildland Nursery

Seeds

Central Utah Seed

Clyde Robin Seed Company Inc

GRANITE SEED

GREAT BASIN SEED

Intermountain Seed Company

Maple Leaf

Maughan Seed Company

Mountain West Seed Company Inc

Paul's Seeds

PLUMMER SEED COMPANY

(see ad on page 209)

Stevenson Intermountain Seed

USDI National Park Service-

Zion National Park Native Plant Nursery

UTAH SEED

\section{VERMONT}

Plants

Elmore Roots Nursery

Grand Isle Nursery

Intervale Conservation Nursery

The Nature Conservancy-Champlain Valley

Native Plant Restoration Nursery

Seeds

AmericanMeadows.com

VIRGINIA

Plants

Botanique

Brent \& Becky's Bulbs 
Nature By Design

Nicholls Gardens

Pinelands Nursery Inc

Riverside Nursery Inc

Sassafras Farm

Snow Mountain Nursery

Summit Nursery

Tidewater Growers

Virginia Dept of Forestry—Augusta Forestry Center

Virginia Dept of Forestry-Garland Gray Forestry Center

Watkins Nurseries Inc

Windy Hill Plant Farm

\section{Seeds}

Joe Brown Native Seeds

Nature By Design

Pinelands Nursery Inc

\section{WASHINGTON}

\section{Plants}

Aldrich Berry Farm \& Nursery Inc

AT James Tree Company

BankSavers Nursery

BLUESTEM NURSERY

Briggs Nursery Inc

Burnt Ridge Nursery

Cascade Biomes

Cloud Mountain Farm

Collector's Nursery

Colville Tribal Forestry Greenhouse

Colvos Creek Nursery

The Country Store \& Gardens

DERBY CANYON NATIVES

Desert Jewels Nursery

Fancy Fronds

Far Pastures Nursery

Fir Run Nursery

Firetrail Nursery

Foliage Gardens

Forest Floor Recovery Nursery

FOURTH CORNER NURSERIES

(see ad on inside cover)

Friendly Natives Plants and Design

Green Man Gardens

The Greenery

Greg Peterson

Hood Canal Nurseries

Judd Creek Nursery

Julius Rosso Nursery Company

Lawyer Nursery Inc

Lewis River Reforestation Inc

Madrona Nursery

Makah Tribal Nursery

METHOW NATIVES

Microseed Nursery

Milestone Nursery

Mount Tahoma Nursery

MsK Rare and Native Plant Nursery

Northwest Native Nursery

Nothing but Northwest Natives

Olympic National Park Native Plant Nursery

Olympic Nursery

Pacific Natives and Ornamentals

Plantas nativa LLC

PLANTAS NATIVA LLC EAST

(see ad on page 207)

PLANTS OF THE WILD
Raintree Nursery

Raven Nursery

The Rhododendron Species Foundation

Rosso Gardens LLC

Silvaseed Company Inc

Sky Native Plant Nursery

Sound Native Plants Inc

Spring Creek Nursery

Stillaguamish Tribe Bank Savers

Storm Lake Growers

SUNBREAK NURSERY COMPANY

Swansons Nursery

The Sweetbriar

Tadpole Haven Native Plants

Tree Frog Farm Inc

USDI National Park Service-

Mount Rainier National Park Native Plant

Nursery

USDI National Park Service-North Cascades

National Park Native Plant Nursery

USDI National Park Service-Olympic National

Park Native Plant Nursery

VIEWCREST NURSERIES

Wabash Farms

WACD PLANT MATERIALS CENTER

(see ad on page 233)

WASHINGTON STATE DEPT OF NATURAL RESOURCES —WEBSTER FOREST NURSERY WATERSHED GARDEN WORKS

Wetlands \& Woodlands Wholesale Nursery

Weyerhaeuser Company_-Rochester Greenhouse WILDLANDS NURSERY

Wildside Growers

Windy Ridge Tree Farm \& Wholesale Nursery

WOODBROOK NURSERY (see ad on page 237)

Seeds

Alpine WildSeed

BFI NATIVE SEEDS (see ad on page 155)

Cascade Biomes

Colville Tribal Forestry Greenhouse

DERBY CANYON NATIVES

Firstline Seeds Inc

FOURTH CORNER NURSERIES

(see ad on inside cover)

Frosty Hollow Ecological Restoration

Grassland West

Greg Peterson

Inside Passage Seeds and Native Plants Services

L\&H Seeds Inc

Landmark Seed Company I

Sun Mountain Native Seed

Lawyer Nursery Inc

METHOW NATIVES

Milestone Nursery

Plantas nativa LLC

PLANTAS NATIVA LLC EAST

(see ad on page 207)

PLANTS OF THE WILD

Rainier Seeds Inc

Silvaseed Company Inc

Stillaguamish Tribe Bank Savers

WASHINGTON STATE DEPT OF NATURAL

RESOURCES-WEBSTER FOREST NURSERY

WATERSHED GARDEN WORKS
WEST VIRGINIA

Plants

Enchanter's Gardens

SUNSHINE FARM AND GARDENS

(see ad on page 223)

West Virginia Division of Forestry-

Clements State Tree Nursery

Seeds

Enchanter's Gardens

SUNSHINE FARM AND GARDENS

(see ad on page 223)

\section{WISCONSIN}

Plants

AGRECOL CORPORATION (see ad on page 149)

APPLIED ECOLOGICAL SERVICES I TAYLOR

CREEK RESTORATION NURSERY

(see ad on page 151)

Aquatic Resources \& Glacial Pond Farms

Arneson's Nursery

Bluestem Farm

Campbell Tree \& Land Company Inc

Chief River Nursery

Detlor Tree Farm

Evergreen Nursery Company Inc

Green Thumb Garden Shoppe LLC

Gress Evergreen Nursery

HILD \& ASSOCIATES INC

Hsu's Ginseng Enterprises Inc

J\&J Transplant Aquatic Nursery

Johnson's Nursery

Klem's Song Sparrow

Krueger's Northwoods Nursery

LAURA'S LANE NURSERY

Lodholz North Star Acres Inc

Lowes Creek Tree Farm

Marshland Transplant Aquatic Nursery

Oak Prairie Farm

Prairie Nursery

Reeseville Ridge Nursery

Retzer Nature Center

Rolling Acres Native Landscape Nursery LLC

Stone Silo Prairie Gardens

Taylor Creek Restoration Nurseries

Wildlife Nurseries Inc

Wisconsin Dept of Natural Resources-

Griffith State Forest Nursery

Wisconsin Dept of Natural Resources-

Hayward State Forest Nursery

Wisconsin Dept of Natural Resources-

Wilson State Forest Nursery

Wood's Edge Farm

Seeds

AGRECOL CORPORATION (see ad on page 149)

APPLIED ECOLOGICAL SERVICES I

TAYLOR CREEK RESTORATION NURSERY

(see ad on page 151)

Hsu's Ginseng Enterprises Inc

Kettle Moraine Natural Landscaping

LAURA'S LANE NURSERY

Oak Prairie Farm

Prairie Frontier

Prairie Future Seed Company

Prairie Nursery

Prairie Seed Source

Reeseville Ridge Nursery

Retzer Nature Center 
Taylor Creek Restoration Nurseries

Wildlife Nurseries Inc

Wisconsin Dept of Natural Resources-

Hayward State Forest Nursery

Wisconsin Dept of Natural Resources-

Wilson State Forest Nursery

WYOMING

Seeds

WIND RIVER SEED

\section{SERVICES}

Clifton Forest \& Appraisal Service Inc Ecological Consultants Inc

JIFFY-PREFORMA (see ad on page 185)

THE SCOTTS MIRACLE-GRO COMPANY

SGS MID-WEST SEED SERVICES INC

(see ad on page 215)

Stuewe \& Sons Inc (see ad on page 219)

SUN GRO HORTICULTURAL

DISTRIBUTION INC (see ad on page 221)

Synergy Resource Solutions Inc

Westchester Community College-

Native Plants Center 